\title{
Environmental Data for Sites in the National Solar Data Network
}

March 1982

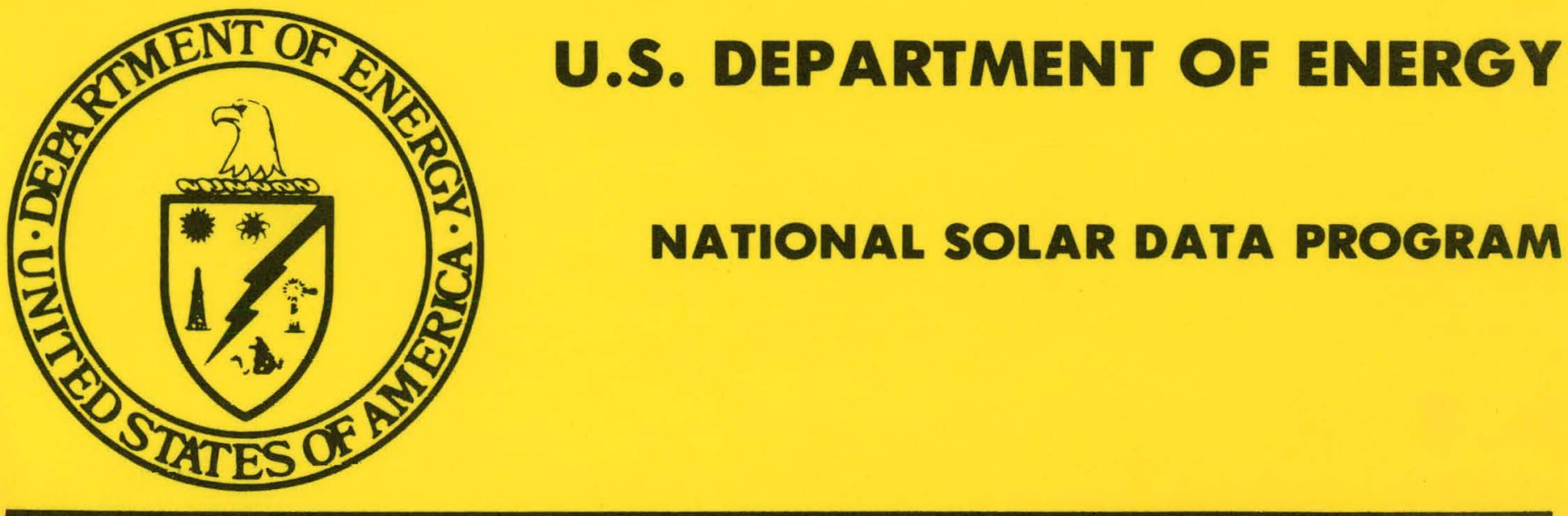




\section{DISCLAIMER}

This report was prepared as an account of work sponsored by an agency of the United States Government. Neither the United States Government nor any agency Thereof, nor any of their employees, makes any warranty, express or implied, or assumes any legal liability or responsibility for the accuracy, completeness, or usefulness of any information, apparatus, product, or process disclosed, or represents that its use would not infringe privately owned rights. Reference herein to any specific commercial product, process, or service by trade name, trademark, manufacturer, or otherwise does not necessarily constitute or imply its endorsement, recommendation, or favoring by the United States Government or any agency thereof. The views and opinions of authors expressed herein do not necessarily state or reflect those of the United States Government or any agency thereof. 


\section{DISCLAIMER}

Portions of this document may be illegible in electronic image products. Images are produced from the best available original document. 


\title{
DISCLAIMER
}

\begin{abstract}
"This report was prepared as an account of work sponsored by an agency of the United States Government. Neither the United States Government nor any agency thereof, nor any of their employees, makes any warranty, express or implied, or assumes any legal liability or responsibility for the accuracy, completeness, or usefulness of any information, apparatus, product, or process disclosed, or represents that its use would not infringe privately owned rights. Reference herein to any specific commercial product, process, or service by trade name, trademark, manufacturer, or otherwise, does not necessarily constitute or imply its endorsement, recommendation, or favoring by the United States Government or any agency thereof. The views and opinions of authors expressed herein do not necessarily state or reflect those of the United States Government or any agency thereof."
\end{abstract}

This report has been reproduced directly from the best available copy.

Available from the National Technical Information Service, U. S. Department of Commerce, Springfield, Virsinia 22161.

Price: Printed Copy A06

Microfiche A01

Codes are used for pricing all publications. The code is determined by the number of pages in the publication. Information pertaining to the pricing codes can be found in the current issues of the following publications, which are generally available in most libraries: Energy Research Abstracts, (ERA); Government Reports Announcements and Index (GRA and I); Scientific and Technical Abstract Reports (STAR); and publication, NTIS-PR-360 available from (NTIS) at the above address. 
MARCH 1982 ENVIRONMENTAL DATA

FOR SITES IN THE

NATIONAL SOLAR DATA NE:TWORK

PREPARED FOR

U.S. DEPARTMEN'I OF ENERGY DE-AC01-79CS30027

R. HASSETT, DOE PROGRAM MANAGER

AND

ARGONNE NATIONAL LABORATORY, G.A. McGINNIS, ANL PROGRAM MANAGER

BY

AUTOMATION INDUSTRIES, INC.

VITRO LABORATORIES DIVISION 14000 GEORGIA AVENUE

SILVER SPRING, MARYIAND 20910

T.T. BRADSHAW, VITRO PROGRAM MANAGER 
INTRODUCTION . . . . . . . . . . . . . . . . . . . . .

ORGANIZATION OF DATA . . . . . . . . . . . . . . . . . . . .

INSTRUMENTATION. . . . . . . . . . . . . . . . . . . . . . 4 4

Insolation. . . . . . . . . . . . . . . . . . . 4

Temperature . . . . . . . . . . . . . . . . . 6

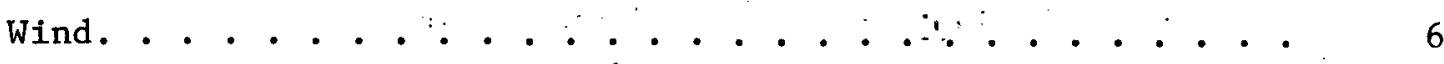

Relative Humidity . . . . . . . . . . . . . . . . 7

OTHER PUBLICATIONS . . . . . . . . . . . . . . . . . 9

ENVIRONMENTAL DATA TABLES. . . . . . . . . . . . . . . . 11

ZONE 1 (Maine, Massachusetts, New Hampshire, Vermont)

Cathedral Square. . . . . . . . . . . . . . . . . 1-1

Contemporary Systems. . . . . . . . . . . . . . . 1-3

Karasek Home. . . . . . . . . . . . . . . . . . . 1-8

Williamson Home . . . . . . . . . ... . . . . 1-10

ZONE 2 (Connecticut, New Jersey, New York, Pennsylvania, Rhode Island)

Environmental Partnership . . . . . . . . . . . . . . 2-1

Gil Harrop. . . . . . . . . . . . . . . . . . . 2-4

Kalin Home. . . . . . . . . . . . . . . . . . . . 2-9

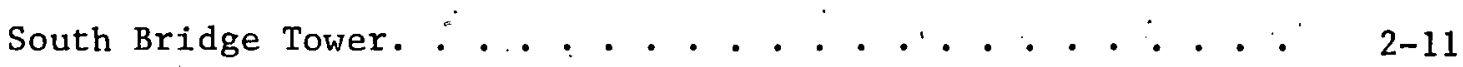

Wood Road School. . . . . . . . . . . . . . . . . 2-13

ZONE 3 (Michigan, Minnesota, North Dakota, South Dakota, Wisconsin)

Arno Kahn . . . . . . . . ... . . . . . . 3-1

EROS Data Center.". . . . . . . . . . . . . . . . 3-6

Isakson Home. . . . . . . . . . . . . . . . . . . 3-8 
ZONE 4 (Idaho, Montana; Wyoming)

No Valid Data

ZONE 5 (Oregon, Washington)

No Valid Data

ZONE 6 (Delaware, Indiana, Kentucky, Maryland, Ohio, Virginia, Washington, D.C., West Virginia)

Carter County..................... . 6-1

Forest City Dillon. .'.................. 6-3

Roberts Home. . . . . . . . . . . . . . . 6-5

Rymark II . . . . . . . ............ . 6-10

Rymark III. . . . . . . . . . . . . . . . . 6-12

Vitro Test Site................... . 6-14

ZONE 7 (Illinois, Iowa, Kansas, Missouri, Nebraska)

Bond Construction . . . . . . . . . . . . . . 7-1

Lo-Cal. . . . . . . . . . . . . . . . . . 7-3

ZONE 8 (Artzona, Colorado, New. Mexico, Utah)

Crown Realty $\#$ I ....................... 8 8-1

Cushing Home. ... ................ 8 8-3

Permaloy Corporation. . . . . . . . . . . . . 8-5

Scottsdale Courthouse... . . . . . . . . . . 8-10

Taos State office Building. . . . . . . . . . . . 8-12

Telluride School. . ... . . . . . . . . . . 8-16 


\section{TABLE OF CONTENTS}

(Continued)

ZONE 9 (California, Nevada)

E1 Toro Library . . . . . . . . . . . . . . . . . 9-1

La Quinta Motor Inn . . . . . . . . . . . . . . 9-3

Oakmead . . . . . . . . . . . . . . . . 9-5

San Anselmo School. . . . . . . . . . . . . ....... . . 9-9

ZONE 10 (Alabama, Georgia, Mississippi, North Carolina,

South Carolina, Tennessee)

No Valid Data

ZONE 11 (Arkansas, Louisiana, Oklahoma, Texas)

Craftsman Enterprises .................. 11-1

ZONE 12 (Florida)

F1orida Solar Energy Center . . . . . . . . . . 12-1 
ENVIRONMENTAL DATA

FOR

SITES IN THE NATIONAL SOLAR DATA NETWORK

\section{INTRODUCTION}

The Department of Energy's Nationa1 Solar Data Network monitors solar energy systems in residential and commercial buildings. Instrumented solar research and development installations employing on-site microcomputers are linked via telephone lines to a Central Data Processing System (CDPS) located at Vitro Laboratories, Silver Spring, Maryland. Vitro collects, archives, analyzes, and reports upon data from this Network to determine the thermal performance of the solar systems.

The Network consists of: (1) sensors which measure key performance parameters at each site; (2) the microprocessor-based Site Data Acquisition Subsystem (SDAS); (3) a modem and telephone transmission interface; (4) the Central Data Processing System (CDPS). The collected data is transferred via telephone communications. At the Central Data Processing Facility, the collected data is processed, analyzed, evaluated and documented. Sensor data are collected and stored on a cassette tape in the SDAS.

Most on-site sensors are interrogated every five minutes and twenty seconds. Solar radiation and other parameters, which are subject to rapid variance, are sampled every 32 seconds and averaged over 320 seconds by the microprocessor. The CDPS communicates with each SDAS on a daily basis and retrieves the stored data. At the conclusion of data retrieval, the SDAS cassette is reset by command from the CDPS to continue data collection.

This report presents environmental information collected at the sites for the reporting month. Certain data are flagged or indicated to be invalid by the use of an asterisk (*). Only those sites for which a significant amount of the data are found to be valid are reported.

ORGANIZATION OF THE DATA

The environmental data for the NSDN are presented in the form of tables for each solar site. The solar sites are grouped into 12 zones, each of which consists of several adjacent states (as shown in Figure 1). The solar energy sites are listed in alphabetical sequence within each zone.

The tables provide available microclimate data for each reporting location in the NSDN as follows:

- Insolation. The insolation table presents total solar radiation in the plane of the collector (Figure 3 ). The total insolation represents the measured value of direct plus diffuse radiation at that orientation. Extraterrestrial radiation is calculated for the same plane of orientation outside the atmosphere. A simple ratio of these two values represents an atmospheric transmission index similar to a cloudiness index. The maximum insolation value occurring in the time-frame of sensor interrogation ( 320 seconds) and its time of occurrence are also shown for each day. 


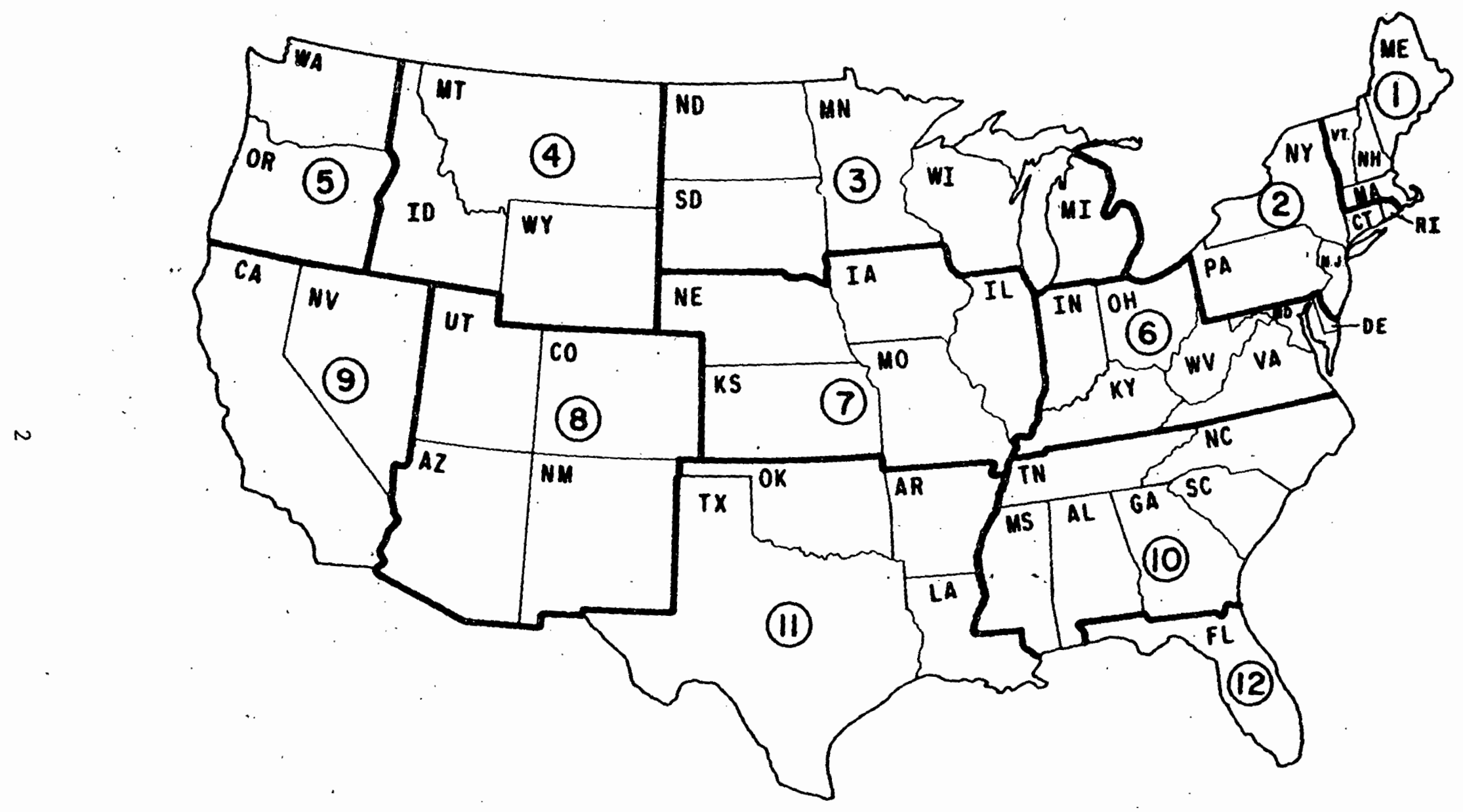

Figure 1. National Solar Data Program Zones 


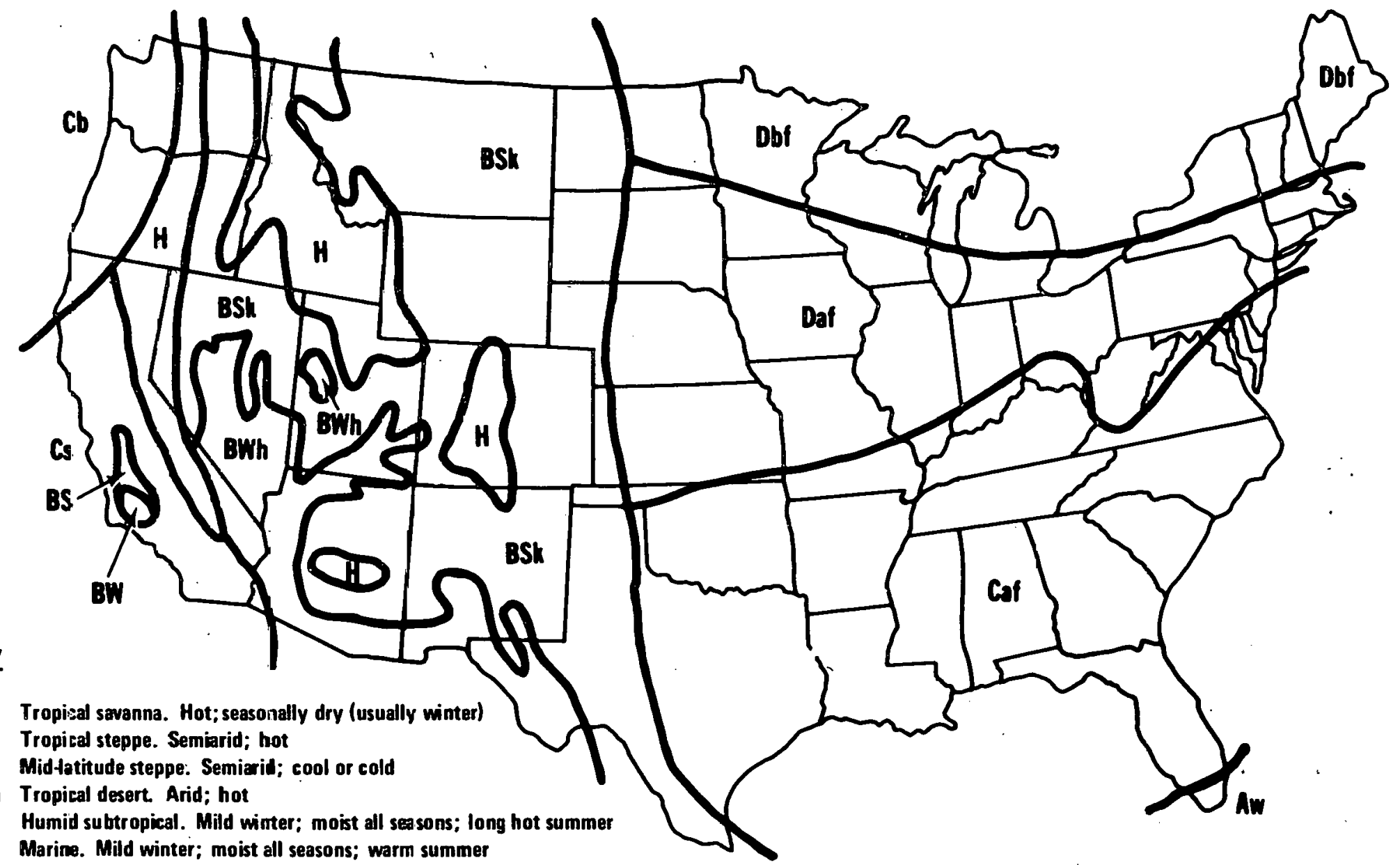

Marine. Mild winter; moist all seasons; warm summer

Coastal Mediterranean. Mild winter; dry summer; short warm summer

Daf Humid continental. Severe vinter; moist all seasons; long, hot summer

Dbf Humid continental. Severe vinter; moist all seasons; short warm summer

H Undifferentiated highland climates

Trewartha, G.T. The Earth's Problem Climates. Uniwersity Wisconsin Press,

Madison, WL, 1961. 
At selected NSDN sites, a shadowband apparatus is deployed with an EPPLEY PSP sensor to measure the diffuse component of incoming solar radiation. The table also shows a daily value for direct beam insolation based on subtraction of the diffuse values from the direct values. These values must be corrected for the amount of sky occluded by the shadowband.

- Temperature. The temperature tables give the average daytime, nighttime, maximum, and inlet-water temperatures at sites with DHW solar systems. Outdoor ambient temperature sensors are mounted near the collectors in appropriate radiation shields. The inlet-water temperatures are mass flow weighted to read values only when flow occurred. Additional tables are presented for some of these NSDN sites at which wind and/or relative humidity data are monitored.

- Wind. All of the passive and some of the active solar sites are equipped with wind sensors. These provide information for two wind tables. The first furnishes wind speed and direction. The second table correlates wind speed with time for each day.

- Humidity. The humidity table provides relative humidity values for day and night. It also gives values for the maximum and minimum humidity for each day and tïmes of occurrence.

A technical discussion of the instruments used for measurement to obtain NSDN data on site environmental conditions follows.

A map illustrating the climate zones is provided in Figure 2 for comparison to NSDN zones and site location.

INSTRUMENTATION

Insolation

EPPLEY PSP pyranometers are used to measure the total solar radiant energy in the plane of the collection subsystem (Figure 3). A pyranometer measures the total amount of solar energy available. Also included are both the direct beam component and the diffuse component if a shadowband instrument is employed at the site to measure the diffuse component. Measurements are given in BTU/ft ${ }^{2}$-day.

The addition of a shadowband to a pyranometer enables the instrument to detect only the diffuse portion of the sunlight by shielding the sensor from the direct rays of the sun (the beam component). The amount of beam radiation available is readily calculated by subtracting the diffuse radiation measurement from the total radiation measured by the unshaded standard pyranometer. This beam radiation measurement is useful when working with focusing solar collectors but must be corrected in user software for the amount of sky occluded by the band apparatus. When using the shadowband pyranometer, the accuracy of measurement depends on the correct adjustment of the shadowband to be certain that the sensor is shielded from the direct rays of the sun. The data reported in these 
summaries have been carefully screened to eliminate publication of invalid data. Thus, this publication may be employed to screen environmental data taken directly from NSDN magnetic tapes.

The EPPLEY PSP pyranometer used includes a circular multijunction wirewound thermopile. The receiver is circular, and coated with Parsons' black lacquer. The instrument has a pair of precision ground and polished hemispheres of Schott optical glass. The sensor head contains a desiccant which can be readily serviced. The clear glass employed transmits wavelengths of 285 to 2,800 nanometers. The temperature dependence is $\pm 1 \%$ over the range of $-4^{\circ} \mathrm{F}$ to $140^{\circ} \mathrm{F}$. The PSP has a response time of one second and a 1inearity of $\pm 5 \%$ over the range of the instrument. Field checking of instruments against calibrated standards indicates very good accuracy and drift resistance under normal deployment conditions.

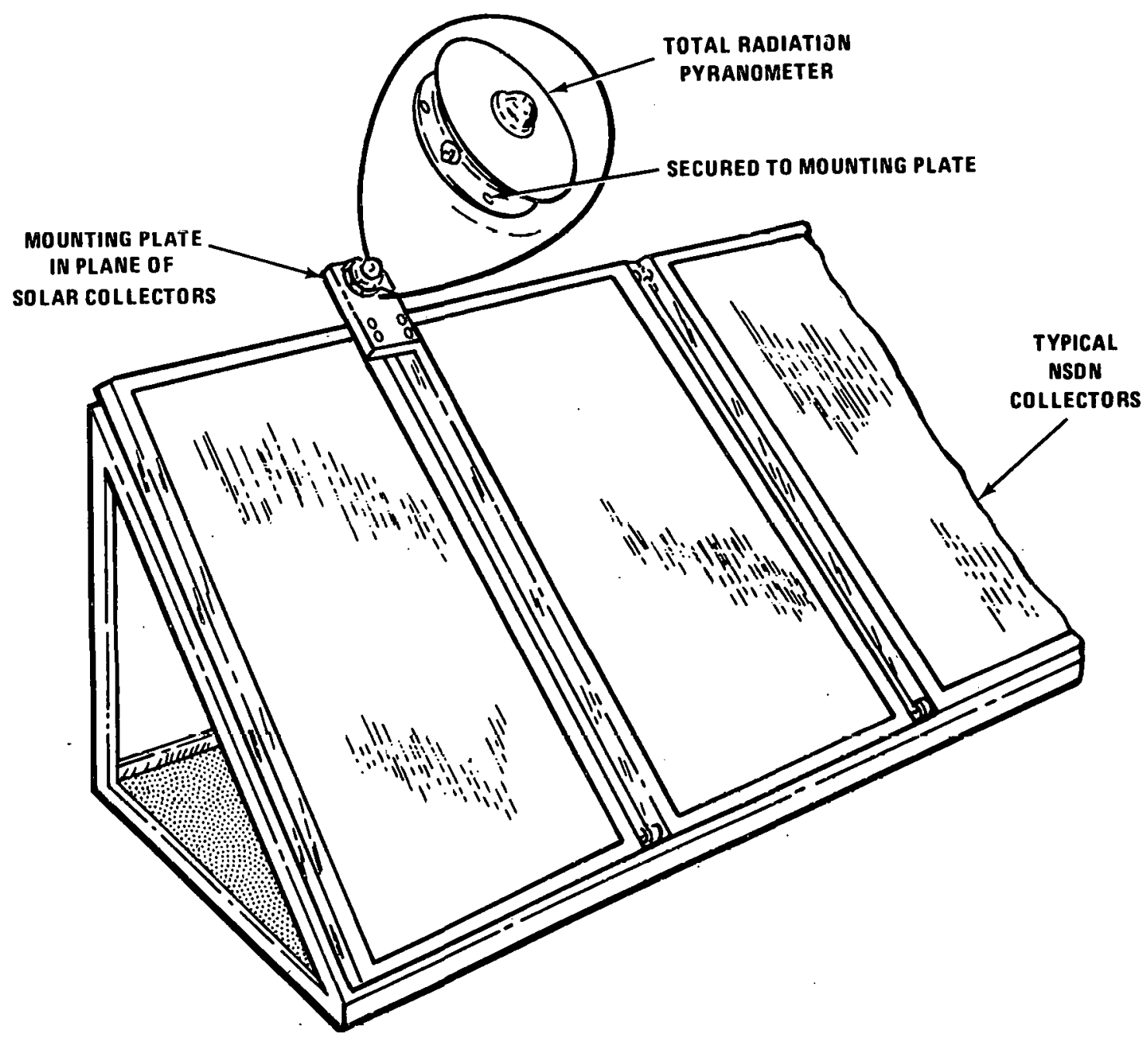

Figure 3. Measurement of Incoming Solar Radiation 


\section{Temperature}

Ambient temperature sensors, (Minco Products S53) platinum Resistance Temperature Detector (RTD), housed in a WeatherMeasure Radiation Shield, (Figure 4) are used to monitor ambient dry bulb temperatures. Sensors are located away from extraneous heat sources. The sensors are sited to be close to the structure and collector array but beyond obvious radiative and convective influences.

The RTD's are connected to a bridge microboard in the SDAS and yield an output signal of 0-100 millivolts proportional to calibrated temperature ranges.

The RTD's are individually calibrated to National Bureau of Standards traceable standards at the factory. In addition, an electrical fivepoint check is done at the site in an attempt to take into account any deviation of the measurement system from nominal values due to sensor wire resistance.

The data processing software at the CDPS takes these checks and calibrations into account, using a third-order polynomial curve fit to convert digital data to temperature engineering units $\left({ }^{\circ} \mathrm{F}\right)$.

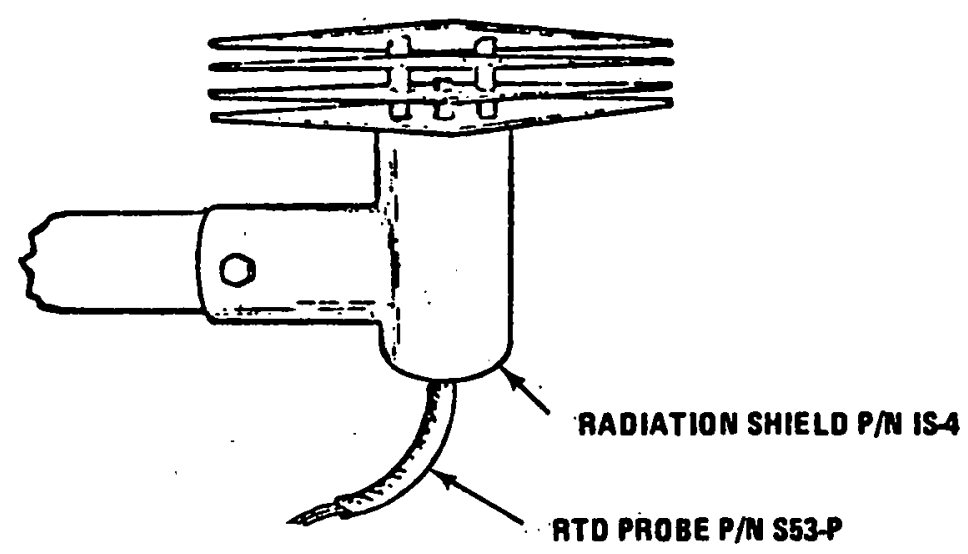

Figure 4. Temperature Sensor

Wind

Where monitored; wind speed and direction are detected by a Model W101-P$\mathrm{DC} / 360$ (or W102-P-DC/360) sensor by the WeatherMeasure Corporation, Sacramento, California (Figure 5).

Wind speed is measured by a four-bladed propeller coupled to a DC generator. The balanced propeller is fabricated from a special low-density, fiberglass-reinforced plastic to yield maximum sensitivity: and strength. Wind speeds below one mile per hour are not reported because these values are below the sensitivity of the instrument. 
Dual-wiper, precious-metal slip rings are used to bring the wind speed analog output ( 15 volts DC at 100 miles per hour) to the base of the sensor from the $0-360^{\circ}$ pivoting Skyvane, which doubles as a direction detector.

Wind direction is measured by means of a dual-wiper 1000-ohm potentiometer housed in the base of the sensor. It is attached to a stainless steel shaft which supports, and rotates with, the upper body assembly.

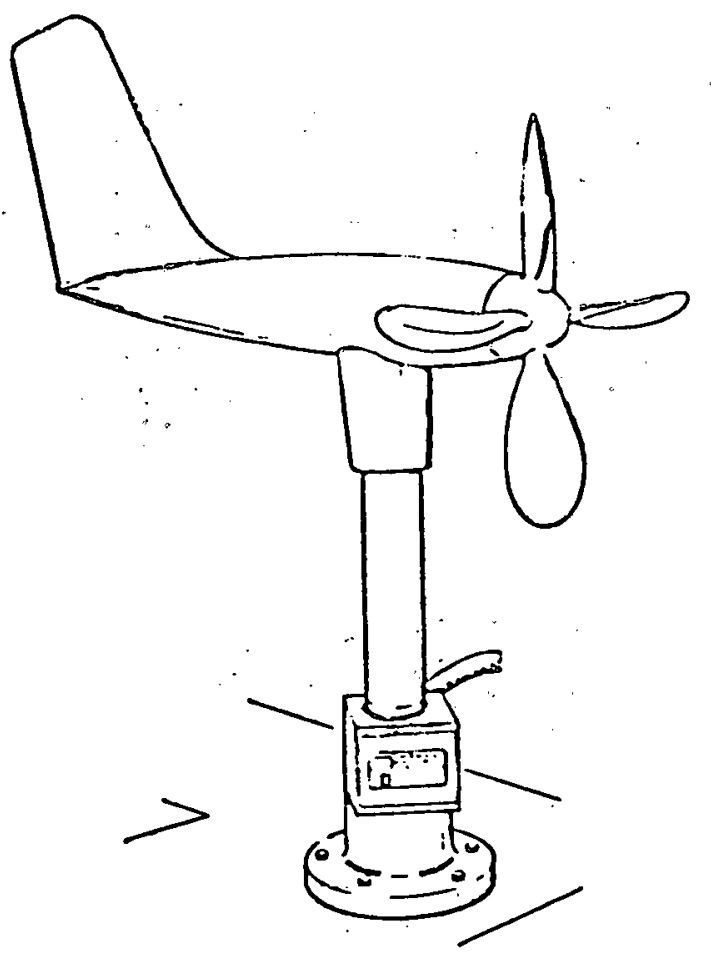

Figure 5. Typical Wind Speed-Direction Sensor

Relative Humidity

Relative humidity is measured by a WeatherMeasure Corporation Model HM111-P/HM14-P sensor (Figure 6). This measurement is of interest in monitoring atmospheric corrosion at active solar sites, and comfort levels at passive solar sites.

This solid-state sensor measures relative humidity over the full range of $0-100 \%$. Response of the sensing element is linear within approximately $1 \%$, from $0-80 \%$ relative humidity.

Sensor analog, output is dependent on the capacitance change of a polymer thin-film capacitor under varying ambient water vapor conditions. The sensor element internally compensates for temperature. A one-micronthick dielectric polymer layer absorbs water molecules through a thin 
metal electrode and causes a capacitance change proportional to water vapor pressure deficit. The thin polymer layer reacts with a time constant of one second to $90 \%$ humidity change at $68^{\circ} \mathrm{F}$. Probe electronics generate linear analog output to the SDAS.

For use outdoors, a sintered filter is used over the probe to reduce the sulphur dioxide absorbed and eliminate small particles. The small pore size of the filter gives good protection to the sensor from acid or particulate degradation. The time constant of the sensor, however, is increased to several seconds.

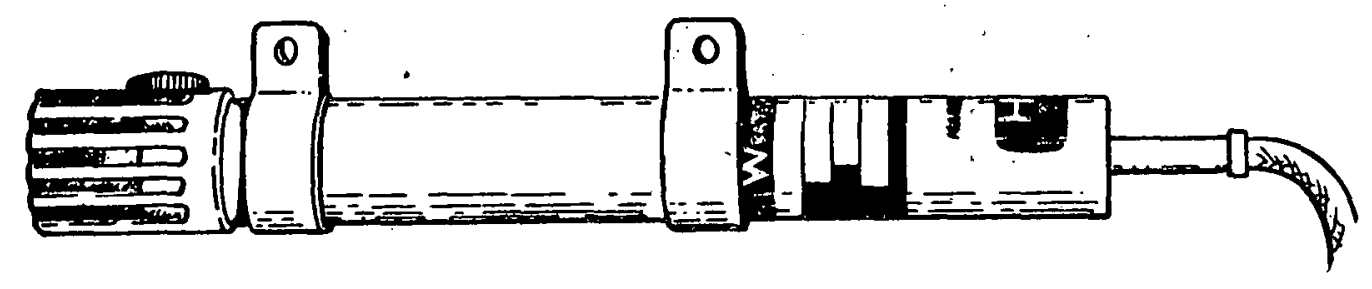

Figure 6. Relative Humidity Probe 
OTHER PUBLICATIONS

These environmental data reports will be issued monthly and will include all sites which are transmitting valid data at the time of publication.

Previous reports are:

SOLAR/0010-78/09, September 1978

SOLAR/0010-78/10, October 1978

SOLAR/0010-78/11, November 1978

SOLAR/0010-78/12, December 1978

SOLAR/0010-79/01, January 1979

SOLAR/0010-79/02, February 1979

SOLAR/0010-79/03, March 1979

SOLAR/0010-79/04, Apri1 1979

SOLAR/0010-79/05, May 1979

SOLAR/0010-79/06, June 1979

SOLAR/0010-79/07, July 1979

SOLAR/0010-79/08, August 1979

SOLAR/0010-79/09, September 1979

SOLAR/0010-79/12, December 1979

SOLAR/0010-80/01, January 1980

SOLAR/0010-80/02, February 1980

SOLAR/0010-80/03, March 1980

SOLAR/0010-80/04, April 1980

SOLAR/0010-80/05, May 1980

SOLAR/0010-80/06, June 1980

SOLAR/0010-80/07, July 1980

SOLAR/0010-80/08, August 1980

SOLAR/0010-80/09, September 1980

SOLAR/0010-80/10, October 1980

SOLAR/0010-80/11, November 1980

SOLAR/0010-80/12, December 1980

SOLAR/0010-81/01, January 1981

SOLAR/0010-81/02, February 1981

SOLAR/0010-81/03, March 1981

SOLAR/0010-81/04, April 1981

SOLAR/0010-81/05, May 1981

SOLAR/0010 $.81 / 06$, June 1981

SOLAR/0010-81/07, July 1981

SOLAR/0010-81/08, August 1981

SOLAR/0010-81/09, September 1981

SOLAR/0010-81/10, October 1981

SOLAR/0010-81/11, November 1981

SOLAR/0010-81/12, December 1981

SOLAR/0010-82/01, January 1982

SOLAR/0010-82/02, February 1982 


\section{THIS PAGE}

\section{WAS INTENTIONALLY LEFT BLANK}


NATIONAL SOLAR DATA NETWORK

ENVIRONMENTAL DATA TABLES

* denotes data missing due to sensor fallure or transmission problems. M.A. denotes data for this parameter not measured at this site.

E denotes value estjmated from eivailable data. 
ZONE 1

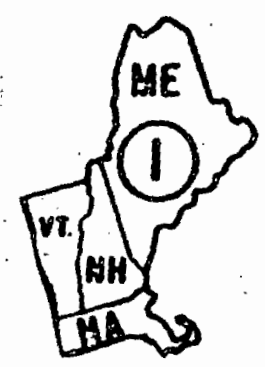


SITE: CATHEDRAL, SQUARE

LATITUDE: $\quad 44.00$ DEG NORTH LONGITUDE: 73.00 DEG WEST
REPORT PERIOD: $82 / 3$

TIME ZONE: EASTERN

ALTITUDE: 200 FEET

\section{DAY TOTAL TOTAL DIFF TOTAL DIR INSOLATION INSOLATION INSOLATION BTU/FT2 BTU/FT2 BTU/FT2 \\ MAXIMUM TOTAL EXTRATERRESTRIAL TTL INS INSOLATION VALUE TIME \\ INSOLAT I
BTU/FT \\ EXT INS}

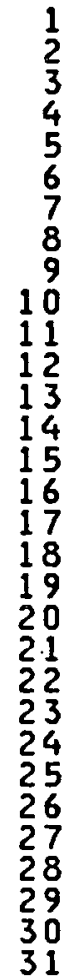

687.

602

1971

758.

1684 .

1098 .

299

1962 .

936
1157

1406 .

$295^{\circ}$

285

2109

$1562^{\circ}$.

1562.
933

1791 .

1997 .

1984.

224

$2064^{\circ}$

$1804^{\circ}$

1821.

1421.

$1469^{\circ}$

2150 .

1782 .

1624

N.A.

N.A.

N.A.

N.A.

$N \cdot A$

N.A.

N.A.

$N \cdot A$.

$N \cdot A$.

N.A.

N.A.

$N \cdot A$

N.A.

N.A.

N.A.

N.A.

N.A.

N.A.

$N \cdot A$.

$N \cdot A$

N.A.

$N \cdot A$

N.A.

$N \cdot A$.

N.A.

N.A.

N.A.

N.A.

N.A

N.A.

N.A.

N.A.

N.A.

N.A.

N.A.

N.A.

$N \cdot A$.

N.A.

N.A.

N.A.

N.A.

N.A.

N.A.

N.A.

$N \cdot A$.

N.A.

N.A.

N.A.

N.A.

209. $11: 45$

$11: 45$

192.

$13: 39$

$209 . \quad 10: 54$

320 .

226.

$13: 16$

$12: 1$

79. $\quad 11: 36$

351.

$13: 11$

320 .

$10: 14$

$11: 56$

$12: 14$

68 .

$102^{\circ}$

$13: 33$

348 .

$13: 13$

$15: 34$

$15: 34$

$13: 28$

$12: 36$

$10: 34$

$12: 18$

$13: 41$

$12: 17$

$13: 3$

$14: 29$

$14: 8$

$13: 19$

$12: 53$

$13: 14$

331 .

328 .

$13: 34$

N.A.

N.A.

N.A.

N.A.

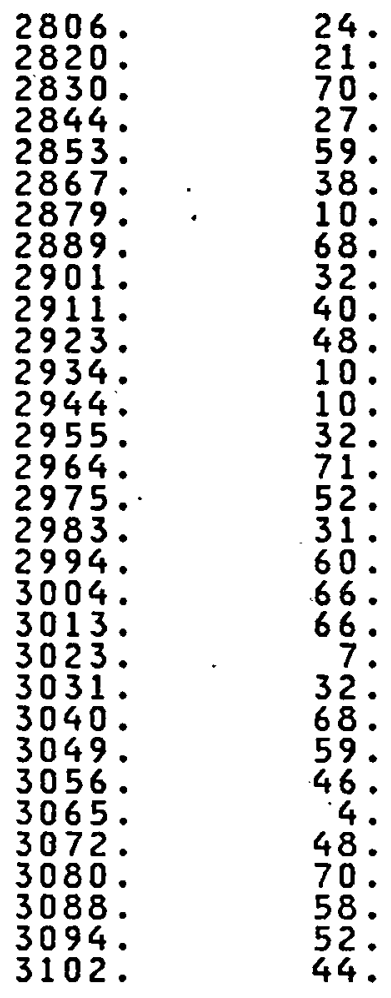

2967.
637.

0.

N.A. 
ENVIRONMENTAL SUMMARY REPORT

SITE: CATHEDRAL SQUARE
BURLINGTON, VERMONT

REPORT PERIOD: $82 / 3$

SITE NUMBER: 168

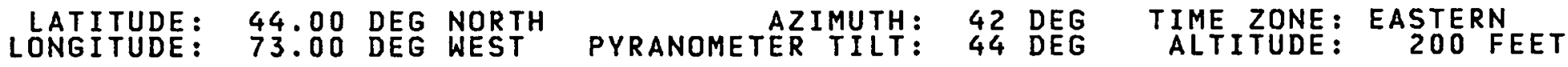

TEMPERATURES IN DEGREES F

\begin{tabular}{|c|c|c|c|c|c|c|}
\hline DAY & $\begin{array}{l}\text { AVERAGE } \\
\text { TEMP }\end{array}$ & $\begin{array}{l}\text { DAYT IME } \\
\text { TEMP }\end{array}$ & $\underset{\text { TEMP }}{\text { NIGHTIME }}$ & $\begin{array}{c}\text { MAX TEMPERATURE } \\
\text { VALUE TIME } \\
\text { HR:MN }\end{array}$ & $\begin{array}{c}\text { MIN TEMPERATURE } \\
\text { VALUE TIME } \\
\text { HR:MN }\end{array}$ & $\begin{array}{l}\text { INLET WATER } \\
\text { TEMPERATURE }\end{array}$ \\
\hline
\end{tabular}

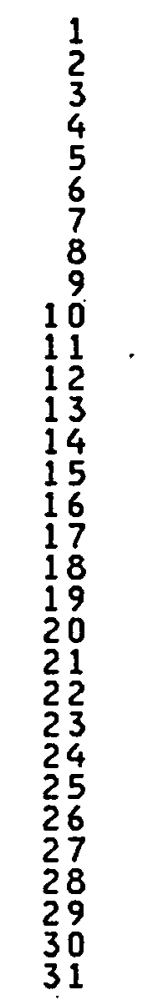

$16:$
$26:$
$13:$
$15:$
$33:$
$31:$
$33:$
$21:$
$17:$
$26:$
$40:$
$40:$
$41:$
$37:$
$33:$
$34:$
$36:$
$40:$
$40:$
39
$35:$
$39:$
$36:$
$41:$
$47:$
$38:$
$17:$
$21:$
$36:$
$47:$
$51:$

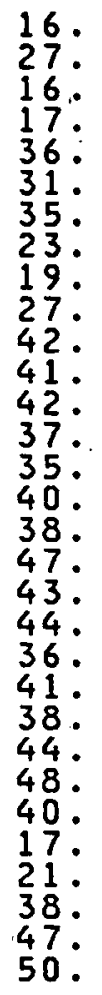

$\frac{16}{2} 5^{\circ}$

11.

$30 \cdot 23: 56$

$31.2: 36$

24. $14: 54$

$40.16: 22$

38 . $13: 53$

$39 \cdot 11: 42$

$28.1 \frac{1}{3}: 42$

$28 \cdot 13: 24$

$25 \cdot 16: 27$

$44 \cdot 20: 39$

44 .

$46: 17: 1$

$42.10: \frac{1}{3}$

$39 \cdot 16: 3$

$49 \cdot 16: 23$

$47 \cdot 14: 22$

45. $17: 0$

$58 \cdot 13: 34$

$49: 16: 36$

42. $10: 2$

$51.12: 23$

$45.14: 20$

45. $14: 29$

$53 \cdot 15: 8$

$57 \cdot 16: 10$

50. $6: 18$

$22 \cdot 15: 1$

33. 16:31

50. 16:10

59. 15:44

56. 15:47

$-0 . \quad 3: 22$

6. $6: 0$

1. $5: 34$

24. $\quad 6: 14$

$28: 23: 58$

9. $23: 56$

4. $0: 28$

$13.6: 14$

$33.55: 16$

35. 1:39

32 . $23: 53$

26 . $23: 52$

19. $5: 33$

$29 \cdot \quad 0: 3$

$25 . \quad 5: 58$

30 . 21:56

29. $5: 29$

35. $4: 58$

29. $23: 17$

27. 5:4.1

40. $6: 25$

$21 \cdot 23: 38$

10 . $5: 52$

23. 5: 3

33 . 5:19

51 .

43. $9: 18$

37.

$38^{\circ}$

38 .

38 .

38 .

MEAN

33.

35.

32 .

38 .

38.

38 .

38

38 .

38 .

38 .

39.

38.

38

38 .

38.

38

38 .

$39^{\circ}$

39.

39.

39 .

38.

STANDARD

DEVIATION

10.1

10.4

10.1

38 .

0.5 
ENVIRONMENTAL SUMMARY REPORT

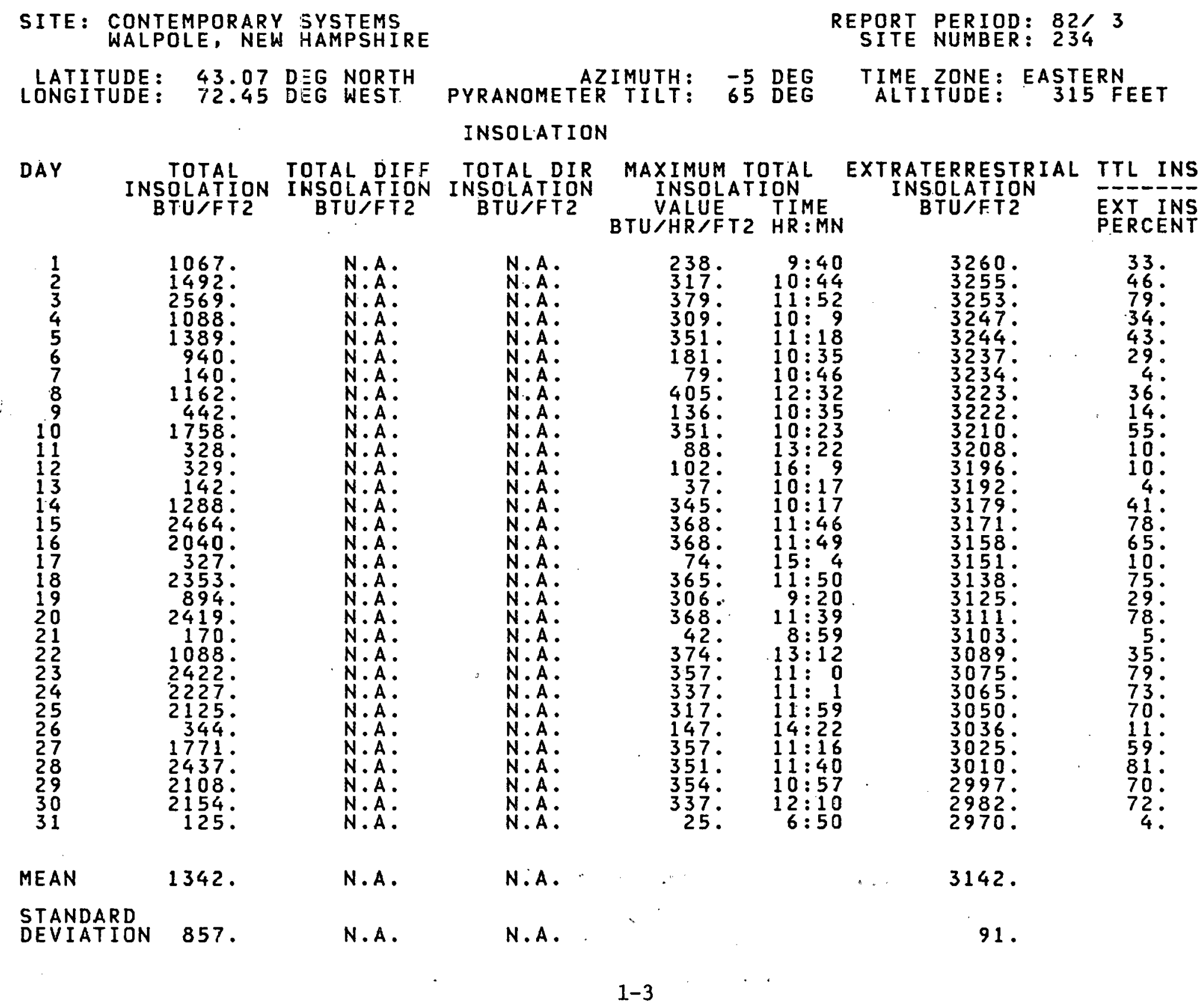


ENVIRONMENTAL SUMMARY REPORT

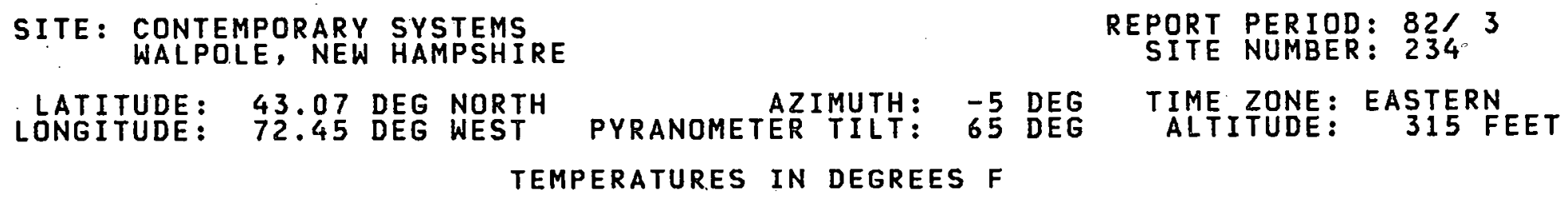

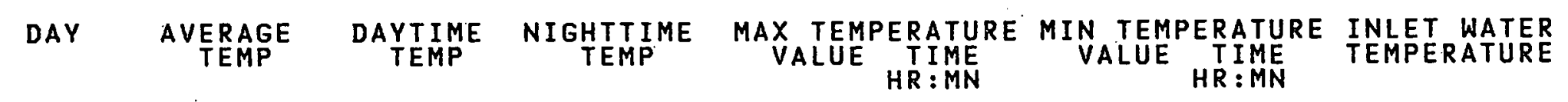

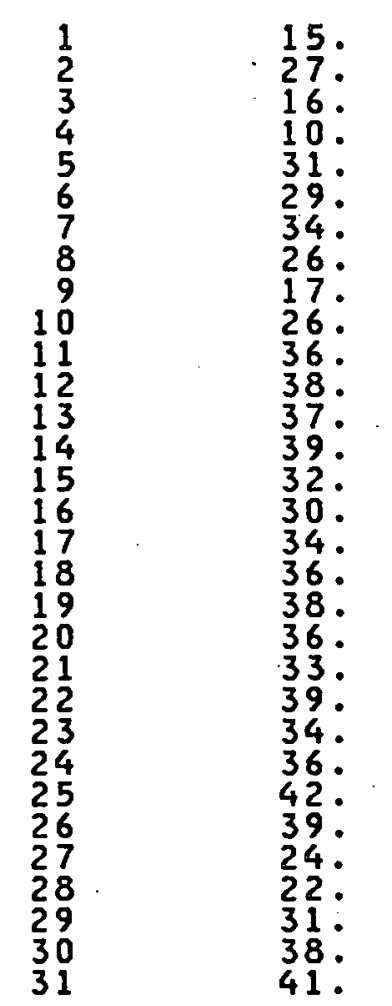

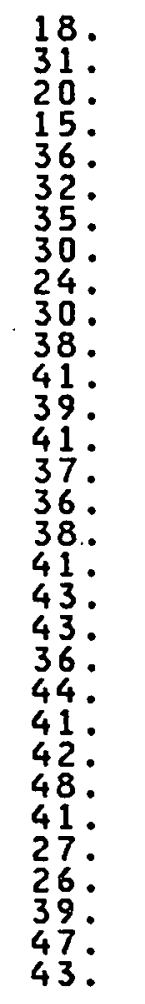

13
13
$23:$
$13:$
$6:$
$26:$
$26:$
$33:$
$23:$
$12:$
$23:$
$35:$
$36:$
$35:$
$37:$
$28:$
$25:$
$31:$
$31:$
$33:$
$29:$
$31:$
$34:$
$28:$
$31:$
$35:$
$37:$
$22:$
$18:$
$22:$
$28:$
$40:$

28. $13: 27$

$35 \cdot 10: 28$

25. $14: 43$

27. $12: 33$

44. $12: 11$

$38.10: 51$

$34.11: 39$

31. $15: 23$

42. $13: 51$

$43.13: 33$

$49 \cdot 15: 47$

$42 \cdot 10: 38$

45. $15: 20$

$43.14: 15$

48. $14: 51$

51: $12: 40$

49 . $12: 5$

51: $14: 3$

40: $11: 31$

49. $15: 4$

46. 15:16

51. 15: 6

58. $14: 23$

45: $8: 26$

32. $13: 50$

$47: 17: 32$

59: 14:24

48. 18:13

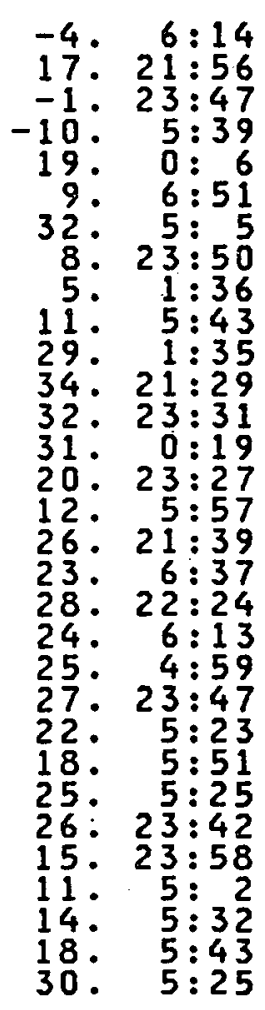

67.

67.

66.

68 .

69.

69.

65.

64.

MEAN

31.

36.

27.

62 .

60.

63.

STANDARD

DEVIATION

8.2

8.5

$8 \cdot 3$

5.0 
ENVIRONMENTAL SUMMARY REPORT

SITE: CDNTEMPORARY SYSTEMS
WALPOLE, NEW HAMPSHIRE

REPORT PERIOD: $82 / 3$ SITE NUMBER: 234

LATITUDE: 43.07 DEG NORTH AZIMUTH: -5 DEG TIME ZONE: EASTERN LONGITUDE: 72.45 DEG. WEST PYRANOMETER TILT: 65 DEG ALTITUDE: 315 FEET

WIND: SPEED IN MPH-DIRECTION IN DEGREES

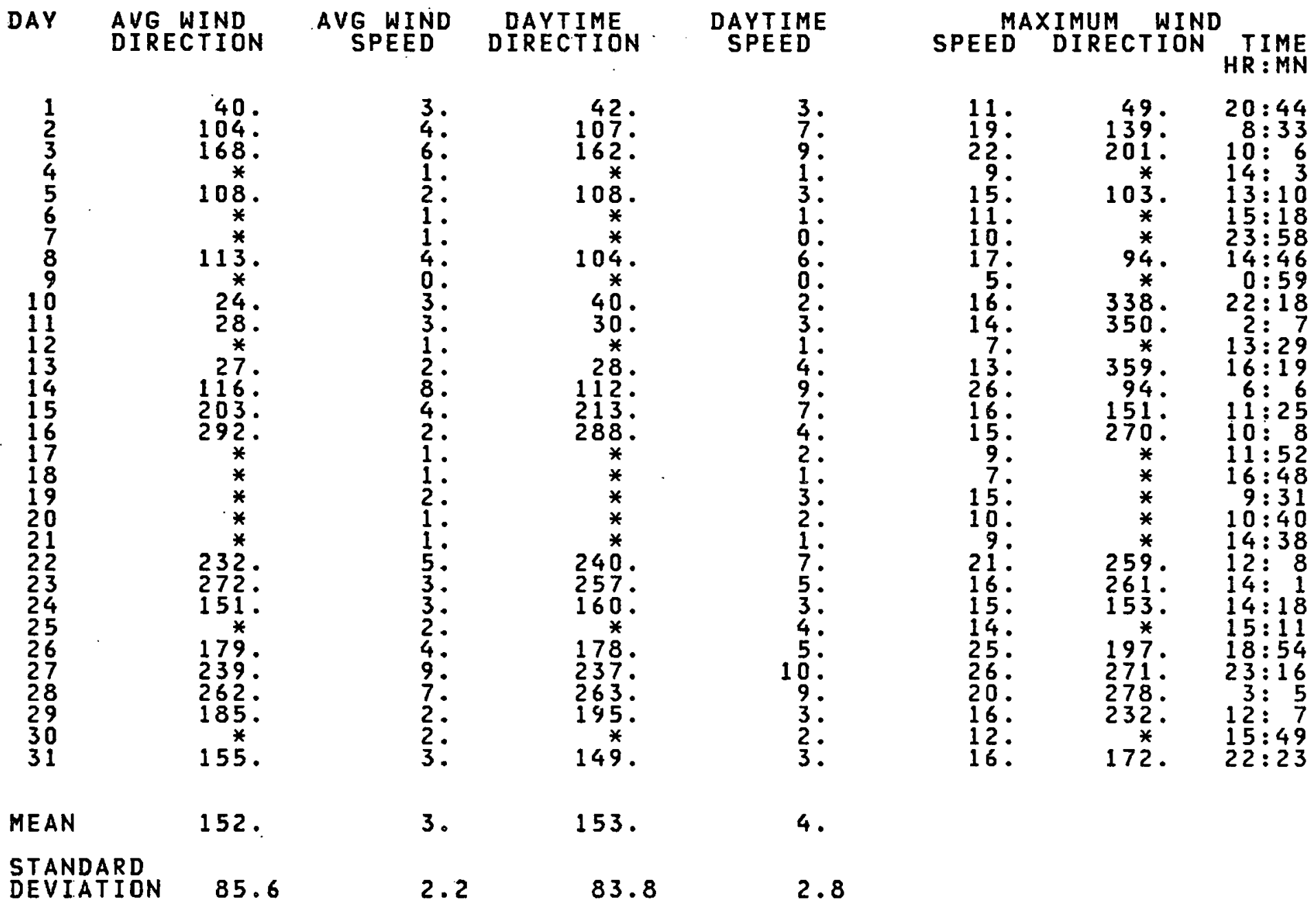


ENVIRONMENTAL SUMMARY REPORT

SITE: CONTEMPORARY SYSTEMS

REPORT PERIOD: $82 / 3$ $\begin{array}{ll}\text { LATITUDE: } & 43.07 \text { DEG NORTH } \\ \text { LONGITUDE: } & 72.45 \text { DEG WEST }\end{array}$ WIND: NUMBER OF HOURS IN EACH VELOCITY INTERVAL

\begin{tabular}{|c|c|c|c|c|c|c|c|c|c|}
\hline DAY & $\begin{array}{l}O-3 \\
\mathrm{MPH}\end{array}$ & $\begin{array}{l}4-7 \\
\text { MPH }\end{array}$ & $\frac{8-12}{M P H}$ & $13-18$ & $19-24$ & $25-31$ & $32-38$ & $\underset{38}{A B O V E}$ & $\begin{array}{c}\text { MISSING } \\
\text { HOURS }\end{array}$ \\
\hline $\begin{array}{r}1 \\
2 \\
3 \\
4 \\
5 \\
6 \\
7 \\
8 \\
9 \\
10 \\
11 \\
12 \\
13 \\
14 \\
15 \\
16 \\
17 \\
18 \\
19 \\
20 \\
21 \\
22 \\
23 \\
24 \\
25 \\
26 \\
27 \\
28 \\
29 \\
30 \\
31\end{array}$ & $\begin{array}{r}19 \\
14 \\
8 \\
24 \\
18 \\
22 \\
23 \\
11 \\
24 \\
15 \\
16 \\
24 \\
19 \\
2 \\
14 \\
19 \\
24 \\
24 \\
19 \\
23 \\
23 \\
10 \\
15 \\
16 \\
18 \\
11 \\
2 \\
7 \\
18 \\
21 \\
19\end{array}$ & $\begin{array}{r}5 \\
6 \\
8 \\
0 \\
6 \\
2 \\
2 \\
1 \\
12 \\
0 \\
5 \\
6 \\
0 \\
5 \\
8 \\
3 \\
3 \\
0 \\
0 \\
5 \\
1 \\
1 \\
9 \\
9 \\
7 \\
6 \\
10 \\
5 \\
7 \\
6 \\
3 \\
4\end{array}$ & $\begin{array}{r}0 \\
4 \\
8 \\
0 \\
0 \\
0 \\
0 \\
1 \\
0 \\
4 \\
2 \\
0 \\
0 \\
13 \\
7 \\
2 \\
0 \\
0 \\
0 \\
0 \\
0 \\
5 \\
0 \\
1 \\
0 \\
3 \\
17 \\
9 \\
0 \\
0 \\
0\end{array}$ & $\begin{array}{l}0 \\
0 \\
0 \\
0 \\
0 \\
0 \\
0 \\
0 \\
0 \\
0 \\
0 \\
0 \\
0 \\
0 \\
0 \\
0 \\
0 \\
0 \\
0 \\
0 \\
0 \\
0 \\
0 \\
0 \\
0 \\
0 \\
0 \\
1 \\
0 \\
0 \\
0\end{array}$ & $\begin{array}{l}0 \\
0 \\
0 \\
0 \\
0 \\
0 \\
0 \\
0 \\
0 \\
0 \\
0 \\
0 \\
0 \\
0 \\
0 \\
0 \\
0 \\
0 \\
0 \\
0 \\
0 \\
0 \\
0 \\
0 \\
0 \\
0 \\
0 \\
0 \\
0 \\
0 \\
0\end{array}$ & $\begin{array}{l}0 \\
0 \\
0 \\
0 \\
0 \\
0 \\
0 \\
0 \\
0 \\
0 \\
0 \\
0 \\
0 \\
0 \\
0 \\
0 \\
0 \\
0 \\
0 \\
0 \\
0 \\
0 \\
0 \\
0 \\
0 \\
0 \\
0 \\
0 \\
0 \\
0 \\
0\end{array}$ & $\begin{array}{l}0 \\
0 \\
0 \\
0 \\
0 \\
0 \\
0 \\
0 \\
0 \\
0 \\
0 \\
0 \\
0 \\
0 \\
0 \\
0 \\
0 \\
0 \\
0 \\
0 \\
0 \\
0 \\
0 \\
0 \\
0 \\
0 \\
0 \\
0 \\
0 \\
0 \\
0\end{array}$ & $\begin{array}{l}0 \\
0 \\
0 \\
0 \\
0 \\
0 \\
0 \\
0 \\
0 \\
0 \\
0 \\
0 \\
0 \\
0 \\
0 \\
0 \\
0 \\
0 \\
0 \\
0 \\
0 \\
0 \\
0 \\
0 \\
0 \\
0 \\
0 \\
0 \\
0 \\
0 \\
0\end{array}$ & $\begin{array}{l}0 \\
0 \\
0 \\
0 \\
0 \\
0 \\
0 \\
0 \\
0 \\
0 \\
0 \\
0 \\
0 \\
1 \\
0 \\
0 \\
0 \\
0 \\
0 \\
0 \\
0 \\
0 \\
0 \\
0 \\
0 \\
0 \\
0 \\
0 \\
0 \\
0 \\
1\end{array}$ \\
\hline AVERAGE & 16.8 & 4.6 & 2.5 & 0.0 & 0.0 & 0.0 & 0.0 & 0.0 & 0.1 \\
\hline TOTAL & 522 & 143 & 76 & 1 & 0 & 0 & 0 & 0 & 2 \\
\hline
\end{tabular}


ENVIRONMENTAL SUMMARY REPORT

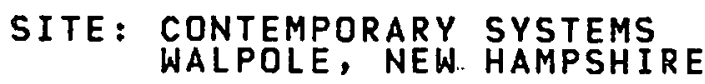

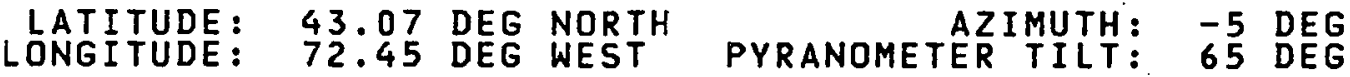
RELATIVE HUMIDITY IN PER CENT

\section{RELATIVE
HUMIDITY}

53.

58.

63.

64 .

58 .

$91^{\circ}$

$71^{\circ}$.

67 .

93.

96 .

$46^{\circ}$

34.

51.

67.

73.

83 .

$55^{\circ}$.

58.

$60^{\circ}$

35 .

26 .

$47^{\circ}$

88 .

63.

MEAN

STANDARD

DEVIATION 18.

NIGHTTIME
REL HUM

64.

69.

$7 \frac{1}{5}$.

66.

68.

74.

80 .

$77^{\circ}$

87 .

97

50 .

43.

69.

85.

84 .

82.
91.
69

73 .

71.

74.

42

33.

64.

88 .

53.

21.

73.

16.
REPORT PERIOD: $82 / 3$

TIME ZONE : EASTERN

ALTITUDE : 315 FEET
HR:MN

\section{$H R: M N$}

\begin{abstract}
87. $20: 33$
\end{abstract}
90. $\quad \begin{aligned} 3: 40 \\ 66 .\end{aligned}$

86. $\quad 23: 36$

89. $\quad 6: 14$

90. $\quad 23: 55$

95. $21: 55$

88. $0: 3$

87. $19 \vdots 1$

88. $\quad 22: 2.0$

$97 . \quad 22: 37$

98. $\quad 8: 39$

$98.1 \quad 19: 26$

68. $\quad 23: 27$

92. $\quad 23: 49$

96. $22: 5$

$96.0 \quad 0 \vdots 8$

95.

94.

$977^{\circ}$

97.

23:49

$90 \quad 5: 28$

92. $\quad 4: 20$

$93.55: 52$

59.

$1: 34$

$23: 40$

$5: 37$

$5: 48$

100. $21: 46$
27. $\quad 11: 10$

25. $\quad 16: 40$

$25 . \quad 16: 40$

37. $\quad 15: 13$

30. $13: 58$

79. $\quad 10: 51$

$38 . \quad 11: 28$

72: $\quad 13: 33$

73. $\quad 16: 14$

$87 . \quad 10: 54$

25. $\quad 15: 41$

16. $17: 14$

14. 14:19

61. $15: 20$

$29.14: 14$

$40 . \quad 16: 5$

$24 . \quad 12: 16$

$58.11: 21$

23. $16: 25$

28. $11: 33$

33. $13: 46$

$16: 14$

$16: 14$

$17 \vdots: 41$

$12: 39$

$14: 18$

67. $8: 26$
$43.12: 38$ 
ENVIRONMENTAL SUMMARY REPORT

SITE : KARASEK HOME

BLACKSTONE, MASSACHUSETTS LOATITUDE: 42.10 DEG NORTH PYRE 71.60 DEG WEST PYNOMETER TILT: INSOLATION

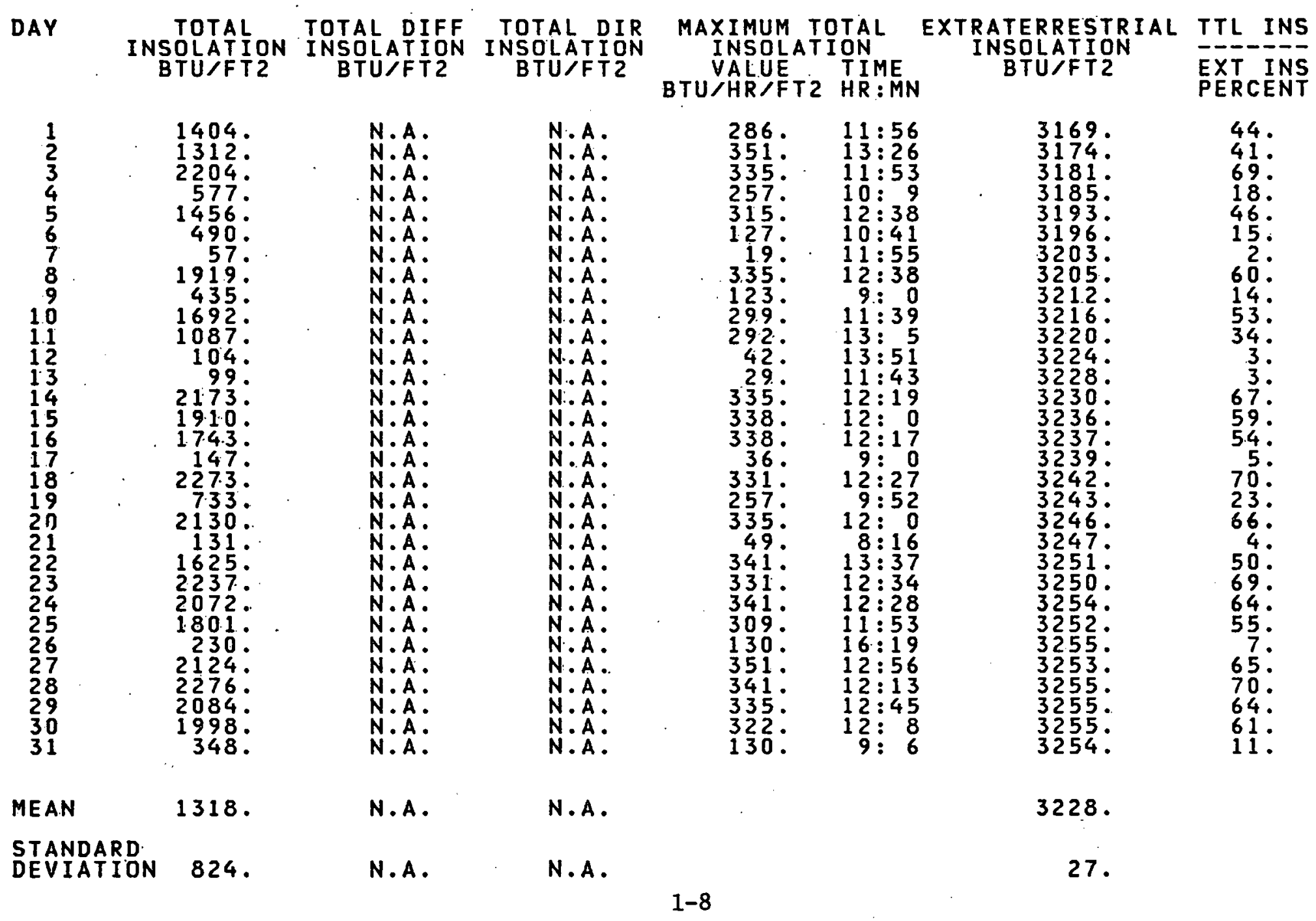

REPORT PERIOD: $82 / 3$
SITE NUMBER: 26 $\begin{array}{ll}20 & \text { DEG TIME ZONE: EASTERN } \\ 45 & \text { DEG }\end{array}$

$\begin{array}{lll}20 & \text { DEG } & \text { TIME ZONE: EASTERN } \\ 45 & \text { DEG } & \text { ALTITUDE: }\end{array}$

\section{RATERRESTRIAL TTL INS \\ PERCENT}

3169 . 44.

3174 .

61. 
ENVIRONMENTAL SUMMARY REPORT

SITE: KARASEK HOME BLACKSTONE, MASSACHUSETTS

REPORT PERIOD: $82 / 3$
SITE NUMBER: 36

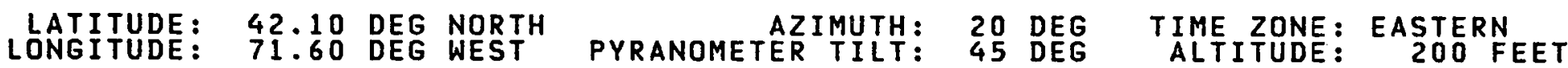

TEMPERATURES IN DEGREES F

\begin{tabular}{|c|c|c|c|c|c|}
\hline DAY & $\begin{array}{l}\text { AVERAGE } \\
\text { TEMP }\end{array}$ & $\begin{array}{c}\text { DAYTIME } \\
\text { TEMPP }\end{array}$ & $\underset{\text { TEMP }}{\text { NI.GHTTIME }}$ & $\begin{array}{c}\text { MAX TEMPERATURE } \\
\text { VALUE TIME } \\
\text { HR:MN }\end{array}$ & $\begin{array}{c}\text { MIN TEMPERATURE } \\
\text { VALUE TIME } \\
\text { HR:MN }\end{array}$ \\
\hline
\end{tabular}

\begin{tabular}{|c|c|c|c|}
\hline $\begin{array}{l}1 \\
2 \\
3 \\
4 \\
5 \\
6 \\
7 \\
8 \\
9 \\
10 \\
11 \\
12 \\
13 \\
14 \\
15 \\
16 \\
17 \\
18 \\
19 \\
20 \\
21 \\
22 \\
23 \\
24 \\
25 \\
26 \\
27 \\
28 \\
29 \\
30 \\
31\end{array}$ & 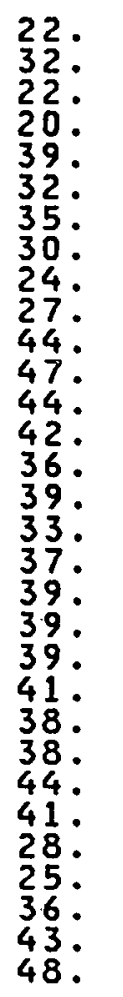 & 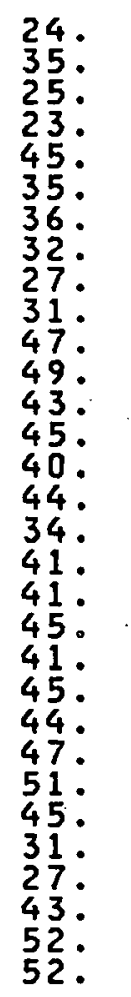 & $\begin{array}{l}21 . \\
30 \\
21 \\
18 \\
35 \\
30 \\
34 \\
28 \\
21 \\
21 \\
41 \\
46 \\
45 \\
39 \\
32 \\
36 \\
32 \\
33 \\
37 \\
33 \\
37 \\
38 \\
33 \\
31 \\
39 \\
38 \\
25 \\
25 \\
23 \\
30 \\
37 \\
44\end{array}$ \\
\hline MEAN & 36. & 39. & 33. \\
\hline $\begin{array}{l}\text { STANDARD } \\
\text { DEVIATION }\end{array}$ & 7.7 & 8.5 & \\
\hline
\end{tabular}

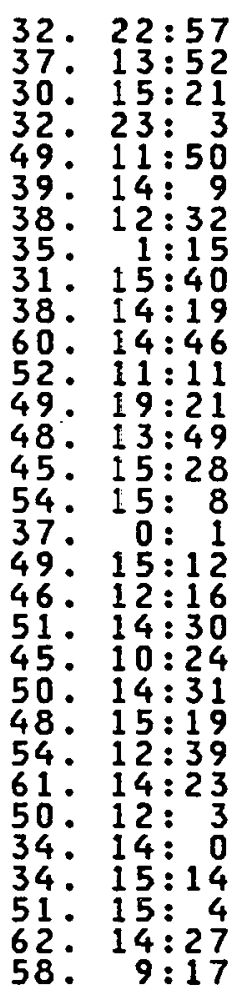

8. $\quad 6: 23$

13: $23: 28$

12.6012

$26: 23: 55$

$24 . \quad \frac{1}{3}: 58$

$33: 26: 25$

$18 \cdot 23: 46$

12. $5: 16$

27. $1: 9$

$42 \cdot 23: 37$

41. 5: 3

$34.23: 20$

26. $4: 29$

$21.26: 88$

21: $5: 30$

$32: 4: 27$

26. $5: 20$

$32: 00: 0$

$35.22: 40$

$25 \cdot 23: 3$

$20 . \quad 5: 27$

$30: 21: 2$

19: $23: 15$

15. $5: 44$

18. $1: 49$

23: $5: 44$

$31 . \quad 5: 17$

59.

56.

64.

57.

62 .

78.

71 .

66 .

56.

61.

54.

49.

61 .

60.

65.

68 .

51 .

58 .

52 .

51 .

54.

60.

54.

59.

6.6 
ENVIRONMENTAL SUMMARY REPORT

SITE: WILLIAMSON HOME IPSWICH, MASSACHUSET.S

LATITUDE: 42.40 DEG NORTH LATITUDE: 42.40 DEG NORTH
LONGITUDE:
70.90 DEG WEST
REPORT PERIOD: $82 / 3$
SITE NUMBER: 241

\begin{tabular}{|c|c|c|c|c|c|c|c|c|}
\hline \multirow[b]{2}{*}{ DAY } & \multicolumn{7}{|c|}{ INSOLATION } & \multirow{2}{*}{\begin{tabular}{l} 
TTL INS \\
\hdashline$\overline{X T}-\frac{-}{\text { INS }}$ \\
PERCENT
\end{tabular}} \\
\hline & $\begin{array}{l}\text { TOTAL } \\
\text { SOLATION } \\
\text { 3TU F T2 }\end{array}$ & $\begin{array}{l}\text { TOTAL DIFF } \\
\text { INSOLATION } \\
\text { BTU/FT2 }\end{array}$ & $\begin{array}{l}\text { TOTAL DIR } \\
\text { INSOLATION } \\
\text { BTU/FT2 }\end{array}$ & $\begin{array}{l}\text { MAXIMUM T } \\
\text { INSOLAT } \\
\text { VALUE } \\
\text { BTU/HR } / F T 2\end{array}$ & $\begin{array}{l}\text { TOTAL } \\
\text { TION } \\
2 \text { TIME } \\
2 \text { HR:MN }\end{array}$ & & $\begin{array}{l}\text { TRATERRESTRIAL } \\
\text { INSOLATION } \\
\text { BTU }\end{array}$ & \\
\hline $\begin{array}{l}1 \\
2 \\
3 \\
4 \\
5 \\
6 \\
7 \\
8 \\
9 \\
10 \\
11 \\
12 \\
13 \\
14 \\
15 \\
16 \\
17 \\
18 \\
19 \\
20 \\
21 \\
22 \\
23 \\
24 \\
25 \\
26 \\
27 \\
28 \\
29 \\
30 \\
31\end{array}$ & 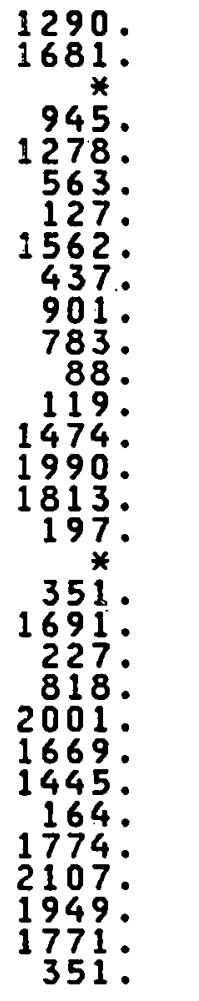 & 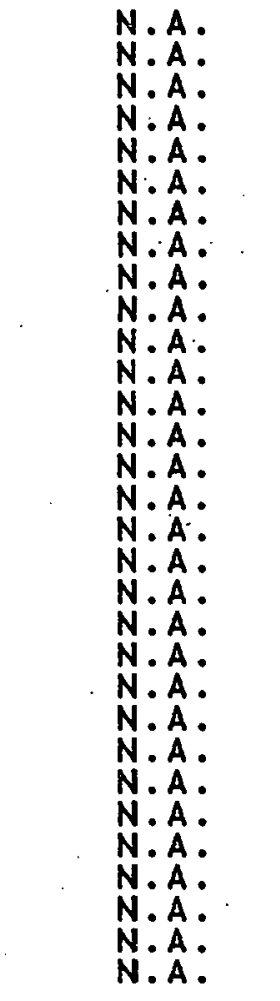 & 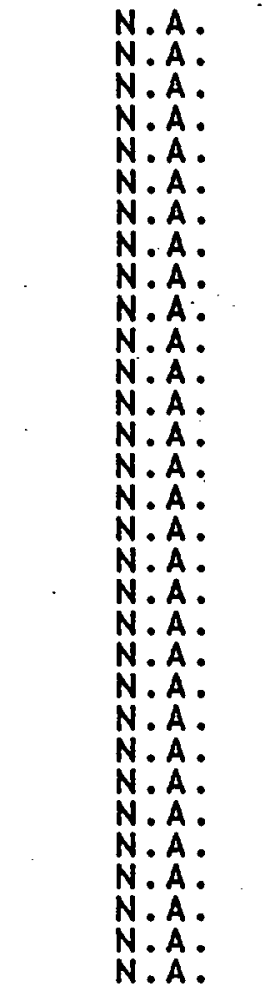 & 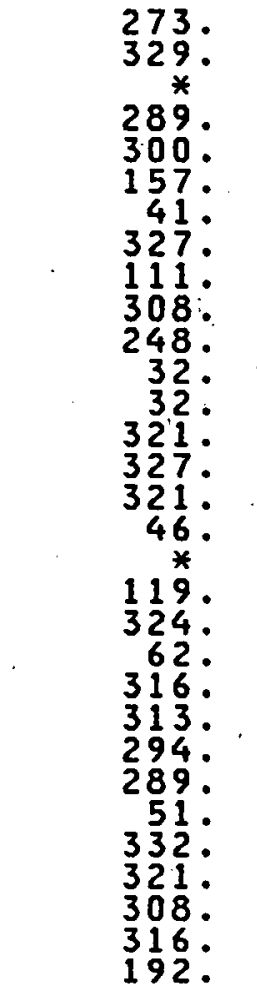 & 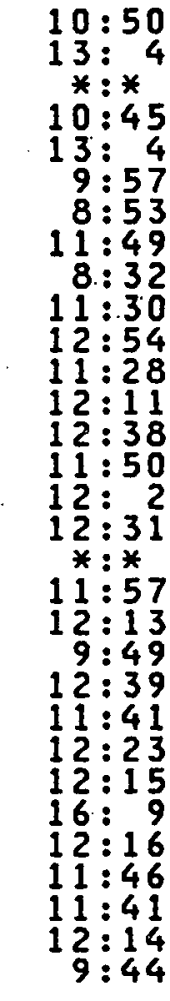 & & 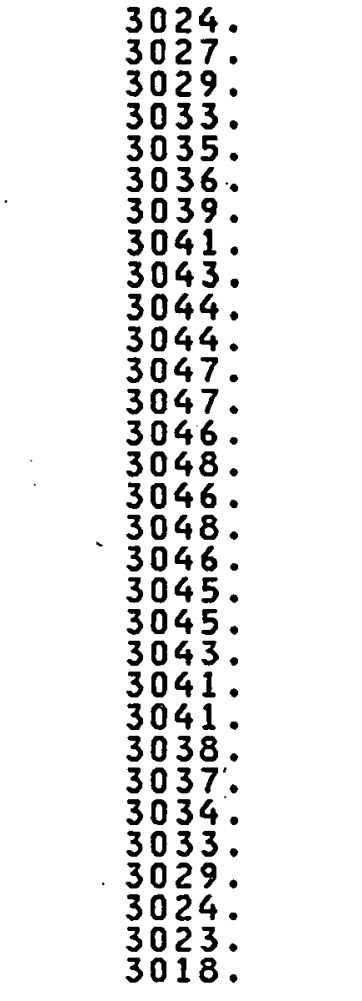 & 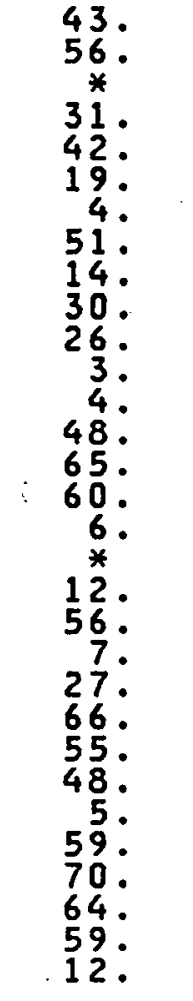 \\
\hline MEAN & 1089. & N.A. & N.A. & & & & 3038. & \\
\hline $\begin{array}{l}\text { STANDARD } \\
\text { DEVIATION }\end{array}$ & 701. & $\mathbf{N} \cdot \mathbf{A}$ & N. A & 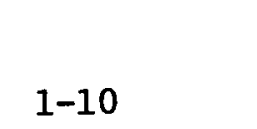 & & & 9. & \\
\hline
\end{tabular}


ENVIRONMENTAL SUMMARY REPORT

SITE: WILLIAMSON HOME

REPORT PERIOD: $82 / 3$
SITE NUMBER: 241

LATITUDE: 42.40 DEG NORTH AZIMUTH: 30 DEG TIME ZONE: EASTERN LONGITUDE: 70.90 DEG WEST PYRANOMETER TILT: 58 DEG ALTITUDE: 100 FEET

TEMPERATURES IN DEGREES F

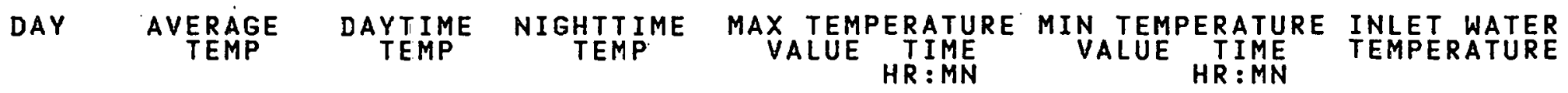

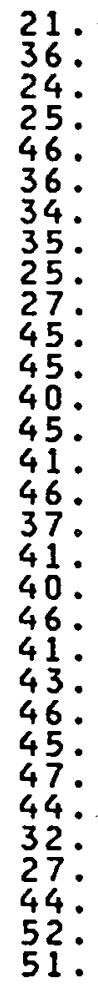

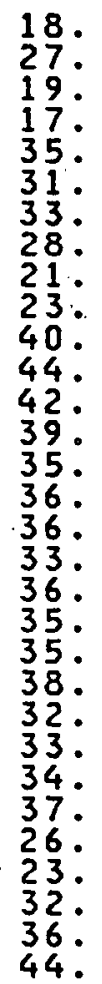

$3 \frac{1}{4} \cdot 13: 57$

31. $14: 45$

$37: 12: 26$

$52: 14: 40$

$43: 11: 23$

$36.10: 13$

41. 11:39

$31 \cdot 12: 48$

$37.13: 54$

$61.14: 52$

$51.0: 33$

46. 20:32

51. $13: 53$

49. $15: 18$

60. 14: 4

41. 1: 0

$47 \cdot 12: 50$

$57^{\circ}$. $12: 18$

$49^{\circ} 12: 18$

$51: 16: 2$

$56 \cdot 15: 25$

56. $9: 54$

65. $13: 41$

$50.13: 18$

39. $13: 30$

$37.15: 19$

56. $14: 58$

$70 \cdot 12: 24$
$58.10: 5$

4. $6: 22$

49.

10. $23: 54$

$-1 . \quad 6: 34$

$28: 23: 49$

32. $\quad 4: 59$

$19.23: 55$

11.6 : 8

30. $1: 54$

40. $21: 52$

$37.21: 50$

$37 . \quad 6: 40$

$35 . \quad 3: 55$

27. $4: 33$

28. 23:59

22 . $5: 56$

28. 23: 3

$29.6: 42$

$33 . \quad 3: 54$

24. $23: 57$

20. $5: 33$

26. $5: 1$

20. $23: 44$

14. $5: 20$

20. $5: 33$

26: $3: 19$

32 . $1: 39$

60.

49.

$50^{\circ}$.

52 .

*

$5 \dot{3}$

53.

52.

55

54.

66.

54.

52.

72 .

71 .

70.

62.

$60^{\circ}$

64.

62.

62
58.

MÉAN

35.

39.

32.

57.

STANDARD

7.6

8.4

7.4

7.3 
ZONE 2

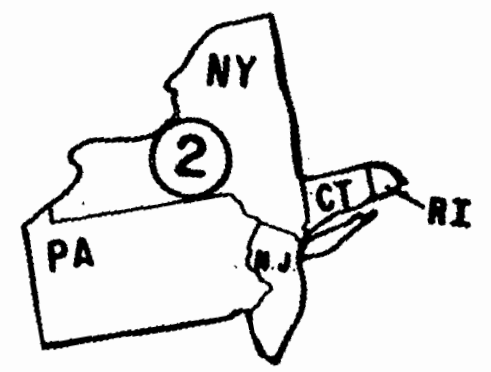


ENVIRCNMENTAL SUMMARY REPORT

SITE: ENVIRONMENTAL PARTNERSHIP
CREAM RIDGE, NEW JERSEY

$\begin{array}{lll}\text { LATITUDE: } & 40.00 \\ \text { LONGITUDE: } & 74: 00 \text { DEG NORTH WEST PYRANOMETER TILT: } 90 \text { DEG }\end{array}$
REPORT PERIOD: $82 / 3$

TIME ZONE: EASTERN

ALTITUDE: 100 FEET.

\section{INSOLATION}

DAY TOTAL TOTAL TCAL HORIZONTAL TOTAL DIR INSOLATION INSOLATION INSOLATION
BTU/FT2

MAXIMUM TOTAL EXTRATERRESTRIAL

TTL INS

INSOLATION VALUE
BTU THR IME

NSOLATION

EXT INS

1
2
3
4
5
6
7
8
9
10
11
12
13
14
15
16
17
18
19
20
21
22
23
24
25
25
26
28
28
30
31

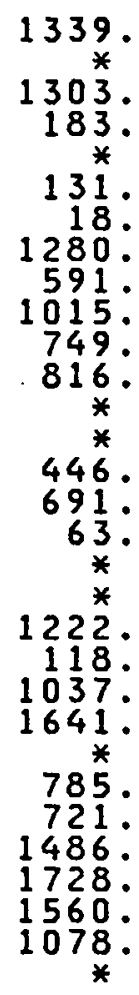

N.A.

N.A.

N.A.

N.A.

N.A.

N.A.

N.A.

N.A.

N.A.

N.A.

N.A.

N.A.

N.A.

N.A.

N.A.

N.A.

N.A.

N.A.

N.A.

N.A.

N.A.

N.A.

N.A.

$N \cdot A$.

$N \cdot A$.

$N \cdot A$.

N.A.

176. $\quad 10: 27$

$23 *$. $\quad$ * $1 * * 2$

55. $15: 10$

13. $\quad$ * $0: *$ : 1

13. $\quad 10: 51$

$215^{\circ} \quad 10: 48$

$180 . \quad 13: 8$

$196 . \quad 13: 0$

$186 . \quad 11: 16$

* $*$ : *

51. $\quad 13: * 25$

$\begin{array}{r}51 . \\ 135 . \quad 8: 35 \\ \hline\end{array}$

$\begin{aligned} 135 . & 8: 58 \\ 0 . & 6: 39\end{aligned}$

* $\quad *: *$

$20 \stackrel{*}{*} .14: * 3$

$10 . \quad 14: 17$

$196 . \quad 11: 6$

$196^{\circ} \quad 11: 68$

$\begin{array}{ll}12 * & * \\ 125 & 14: 37 \\ 157 & 16: 42\end{array}$

215. $14: 39$

205. $\quad 13: 56$

199. $14: 6$

$131 . \quad 9: 36$

N.A.

2639.47.

2624.

$2587^{\circ}$.

$2570^{\circ}$

2551.

2515 .

2496.

2475.

2435

2411 .

2390.

2366 .

2344

2322 .

2273.

2250 .

2226.

$2170^{\circ}$.

2141.

2112 .

2083.

2052 .

2023

$1966^{\circ}$.

1938.

$5 \stackrel{*}{5}$.

MEAN

717.

870 .

N.A.

2324 .

214. 
ENVIRONMENTAL SUMMARY REPORT

SITE: ENVIRONMENTAL PARTNERSHIP
CREAM RIDGE, NEW JERSEY

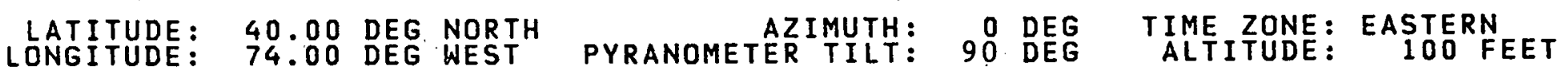

TEMPERATURES IN DEGREES F
REPORT PERIOD: $82 / 3$ SITE NUMBER: 149

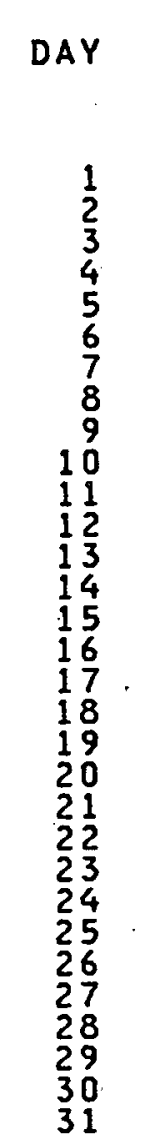

MEAN

STANDARD
DEVIATION

\section{AVERAGE
TEMP}

DAYTIME

NIGHTTIME

MAX TEMPERATURE MIN
VALUE TIME HR:MN

IN TEMPERATURE

INLET WATER
TEMPERATURE

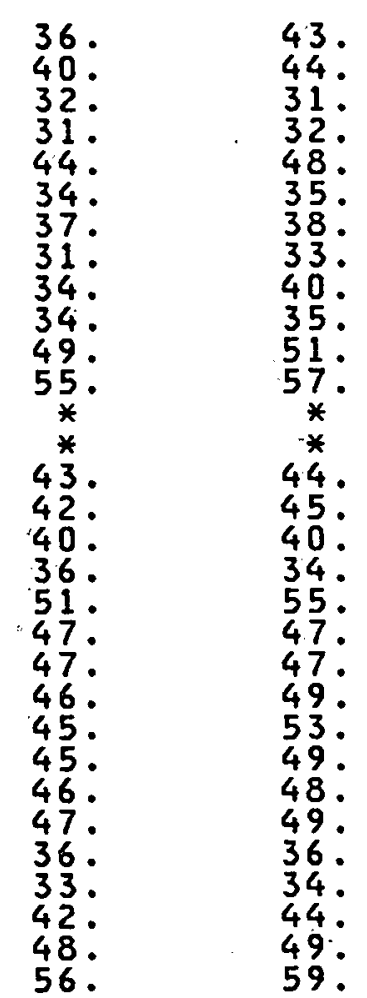

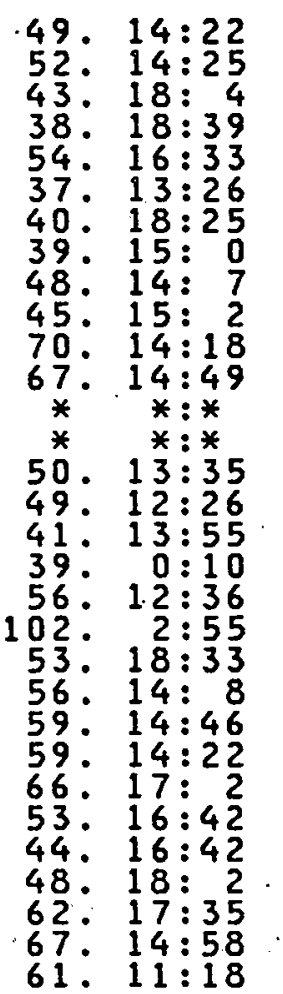

61: $11: 18$

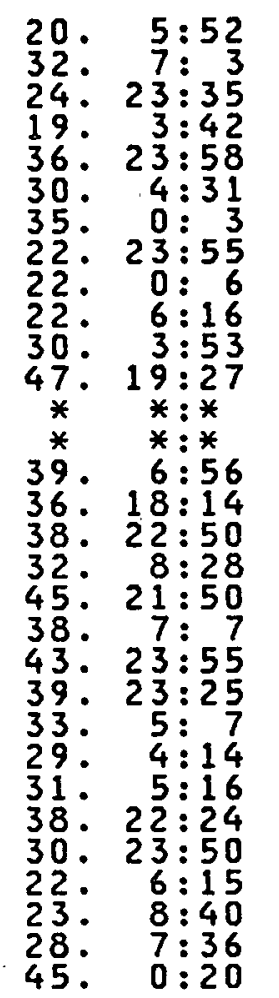

56.

51.

52 .

58 .

55 .

55 .

53 .

53.

33.

45 .

42

38.

38.

47 .

46.

44.

41.

44.

$355^{\circ}$

42.

46
54.

40 .

6.7

7.9

$7 \cdot 1$

44.

7.9

6.7 
ENVIRONMENTAL SUMMARY REPORT

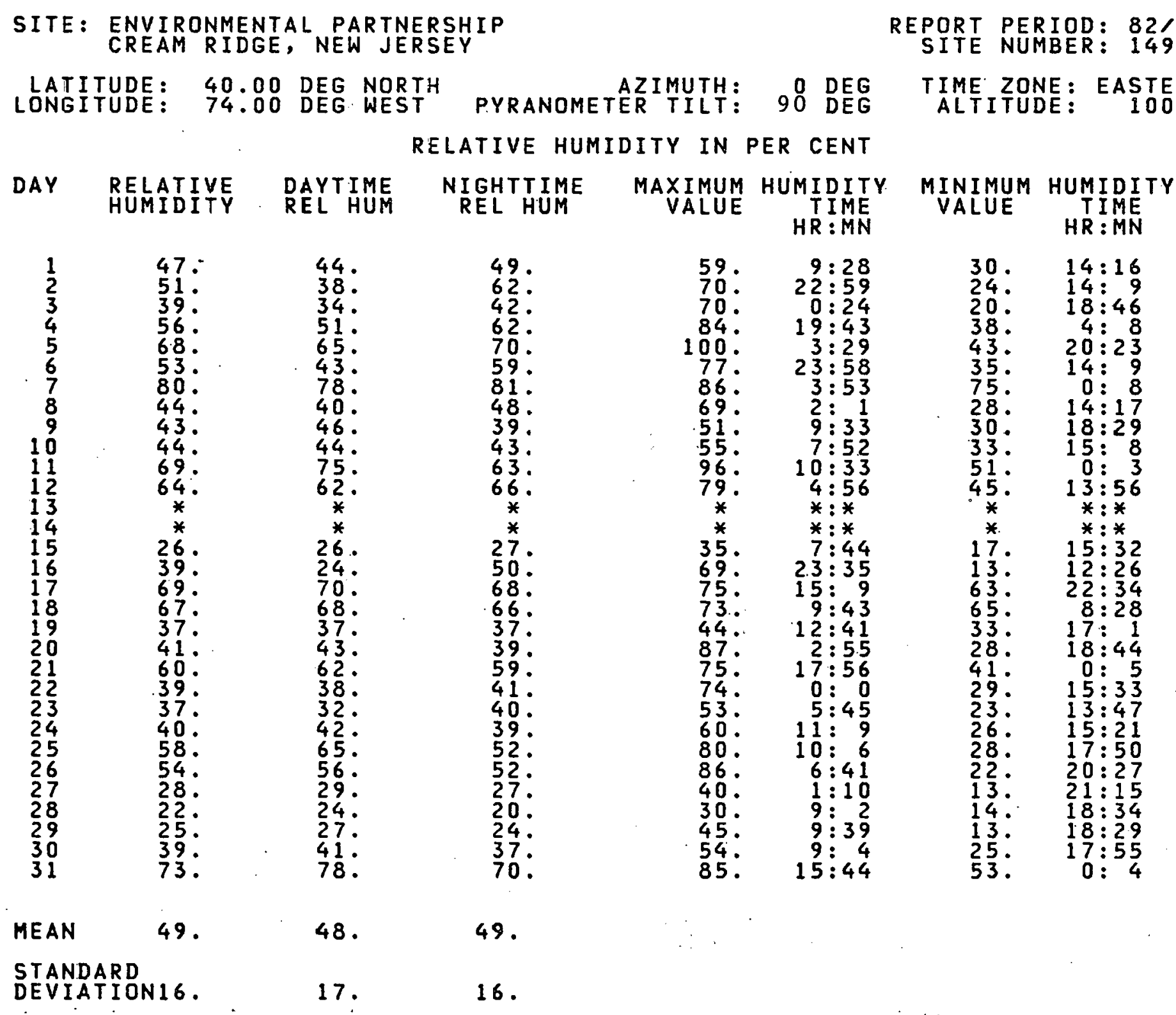


ENVIRONMENTAL SUMMARY REPORT

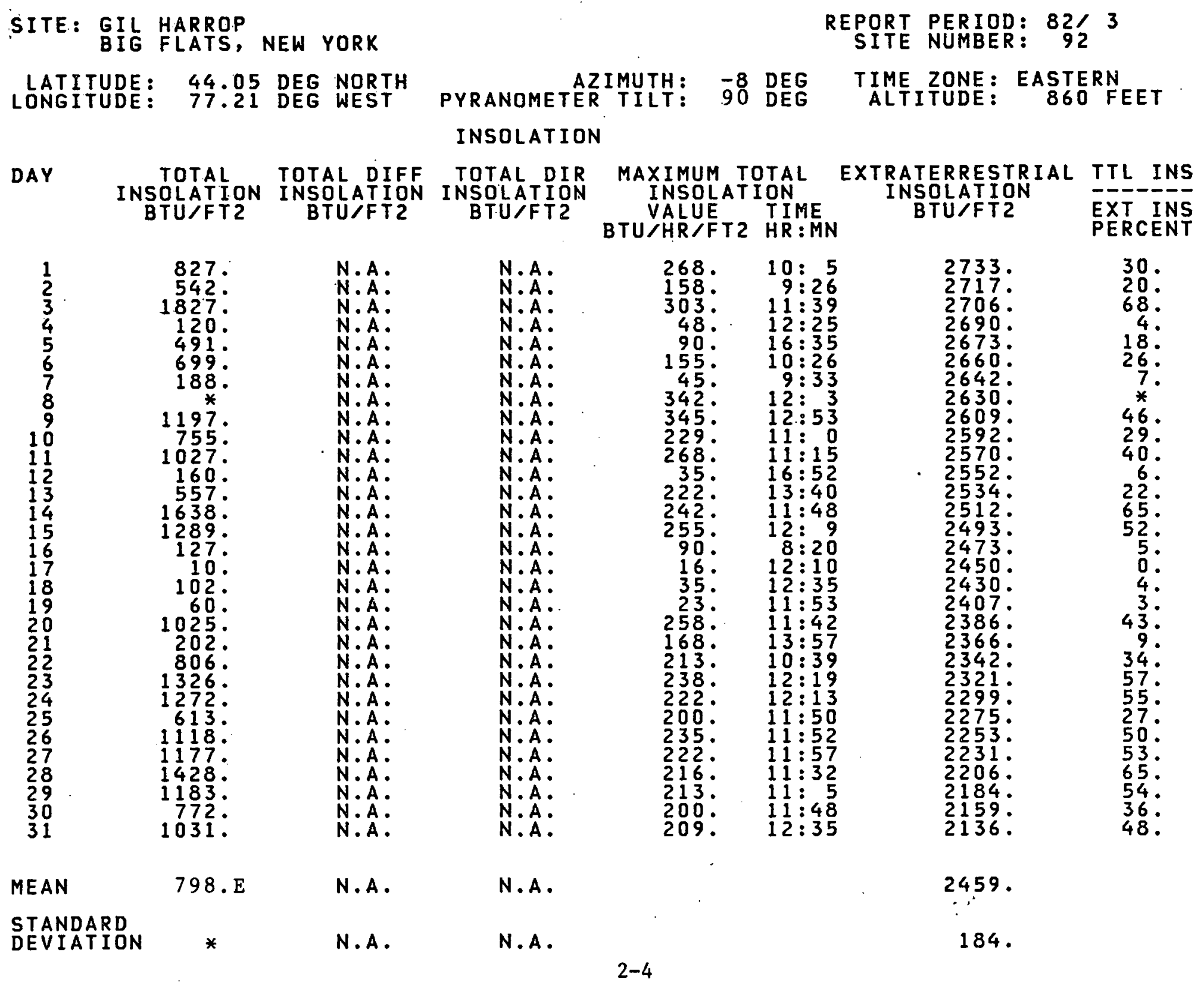


SITE: GIL HARROP
BIG FLATS, NEW YORK

REPORT PERIOD: $82 / 3$
SITE NUMBER: 92

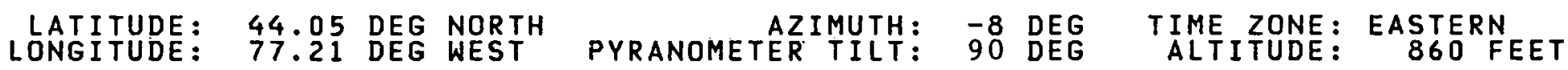

TEMPERATURES IN DEGREES F

\begin{tabular}{|c|c|c|c|c|c|c|c|}
\hline DAY & $\begin{array}{c}\text { AVERAGE } \\
\text { TEMP }\end{array}$ & $\begin{array}{l}\text { DAYTIME } \\
\text { TEMP }\end{array}$ & NIGHTTIME & $\begin{array}{c}\text { MAX TEMP } \\
\text { VALUE }\end{array}$ & $\begin{array}{l}\text { ERATURE } \\
\text { TIME } \\
\text { HR: MN }\end{array}$ & $\begin{array}{c}\text { MIN TEMPERATURE } \\
\text { VALUE } \\
\text { TIIME } \\
\text { HRN }\end{array}$ & $\begin{array}{l}\text { INLET WATER } \\
\text { TEMPERATURE }\end{array}$ \\
\hline $\begin{array}{l}1 \\
2 \\
3 \\
4 \\
5 \\
6 \\
7 \\
8 \\
9 \\
10 \\
11 \\
12 \\
13 \\
14 \\
15 \\
16 \\
17 \\
18 \\
19 \\
290 \\
21 \\
22 \\
23 \\
24 \\
25 \\
26 \\
27 \\
28 \\
29 \\
30 \\
31\end{array}$ & 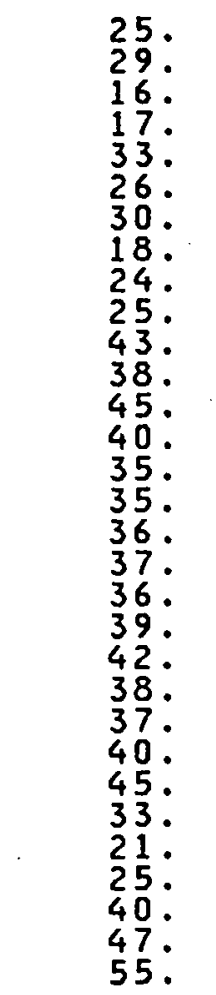 & $\begin{array}{l}31 . \\
32 . \\
22 . \\
20 . \\
36 . \\
29 . \\
33 . \\
23 . \\
30 . \\
28 . \\
50 . \\
42 . \\
51 . \\
44 . \\
40 . \\
37 . \\
37 . \\
39 . \\
38 . \\
44 . \\
46 . \\
42 . \\
46 . \\
49 . \\
52 . \\
36 . \\
23 . \\
33 . \\
51 . \\
57 . \\
61 .\end{array}$ & 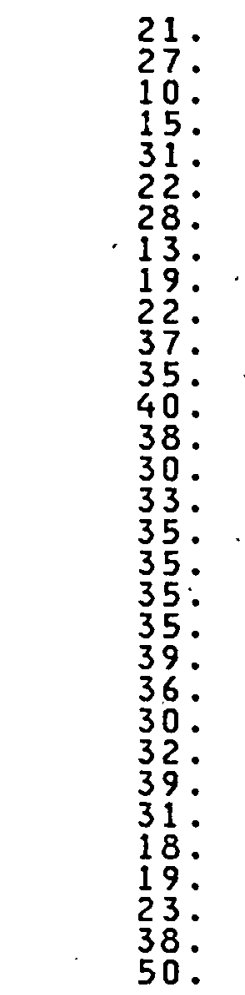 & 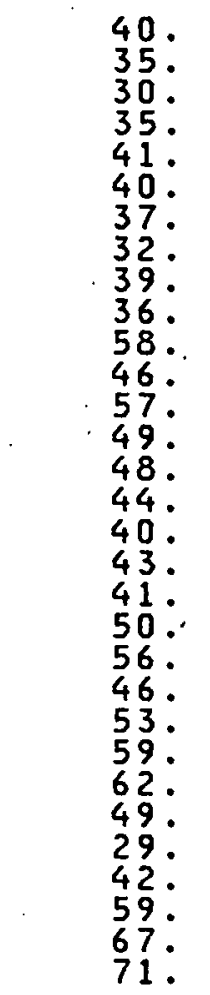 & $\begin{array}{r}13: 1 \\
0: 52 \\
13: 57 \\
22: 49 \\
5: 18 \\
14: 10 \\
9: 48 \\
16: 13 \\
11: 1 \\
13: 40 \\
10: 16 \\
13: 40 \\
15: 5 \\
15: 5 \\
14: 49 \\
11: 29 \\
12: 15 \\
15: 15 \\
14: 22 \\
14: 54 \\
14: 50 \\
16: 10 \\
13: 39 \\
14: 58 \\
13: 47 \\
15: 21 \\
14: 50 \\
15: 16 \\
16: 17 \\
11: 48 \\
14: \\
14\end{array}$ & 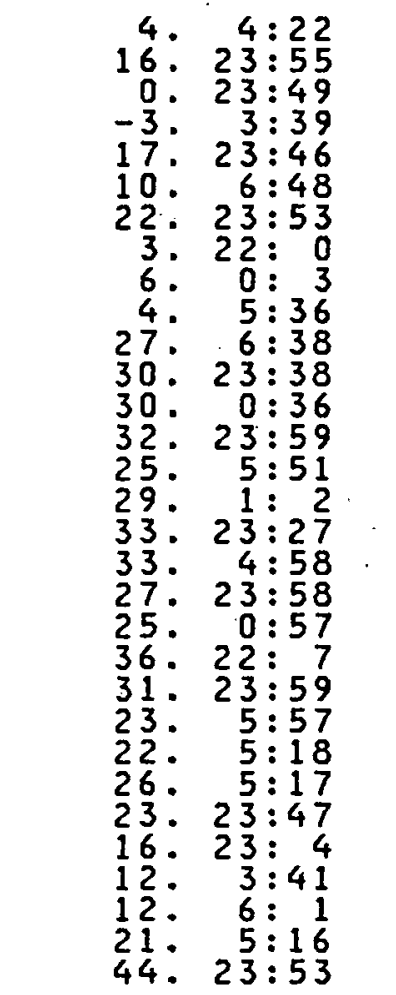 & 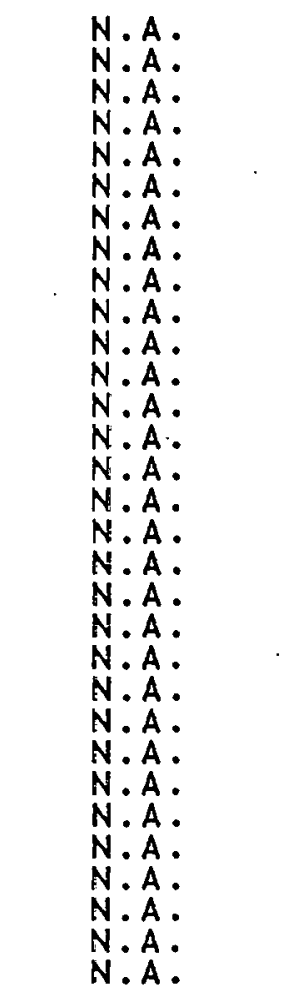 \\
\hline MEAN & 34. & 39. & 29. & $\therefore$ & & & N.A. \\
\hline $\begin{array}{l}\text { STAN } \\
\text { DEVI }\end{array}$ & 9.5 & 10.6 & 9.2 & & . & $\therefore$ & N.A. \\
\hline
\end{tabular}


ENVIRONMENTAL SUMMARY REPORT

SITE: GIL HARROP
BIG FLATS, NEW YORK

REPORT PERIOD: $82 / 3$
SITE NUMBER: 92

LATITUDE: 44.05 DEG NORTH AZIMUTH: -8 DEG TIME ZONE: EASTERN LONGITUDE: 77.21 DEG WEST PYRANOMETER TILT: 90 DEG ALTITUDE: 860 FEET

WIND: SPEED IN MPH-DIRECTION IN DEGREES

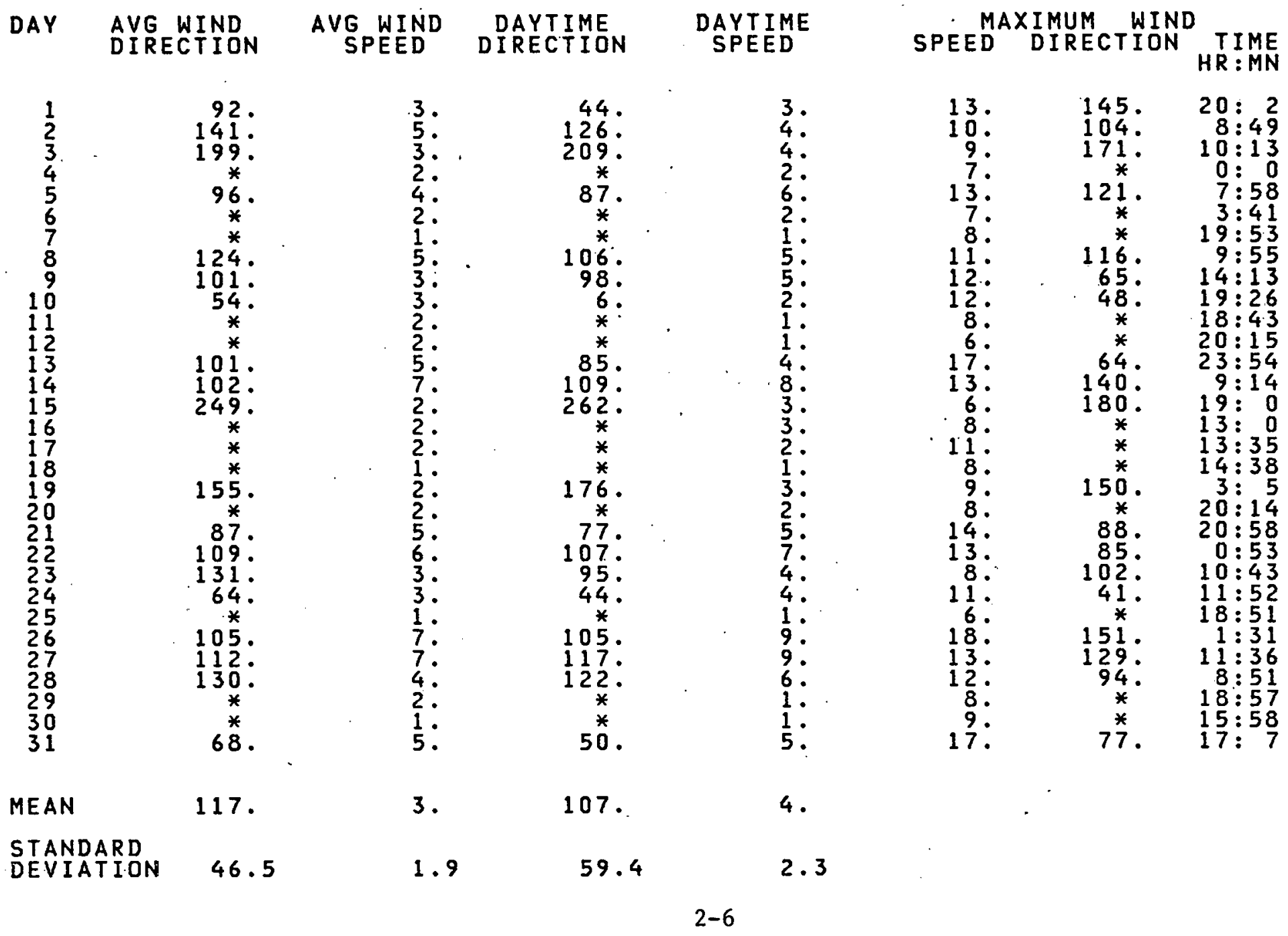


ENVIRONMENTAL SUMMARY REPORT

SITE: GIL HARROP

BIG FLATS, NEW YORK

LATITUDE : 44.05 DEG NORTH $\begin{array}{ll}\text { LATITUDE: } & 44.05 \\ \text { LONGITUDE : } & 77.21 \text { DEG WORTH }\end{array}$

WIND: NUMBER OF HOURS IN EACH VELOCITY INTERVAL
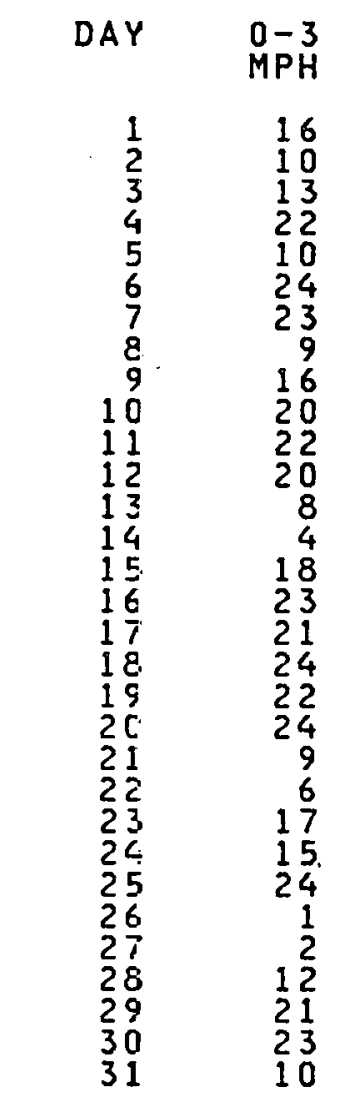

4-7

$$
\begin{aligned}
& 8-12 \\
& M P H
\end{aligned}
$$$$
13-18
$$$$
19-24
$$$$
25-31
$$$$
32-38
$$

ABOVE

MISSING

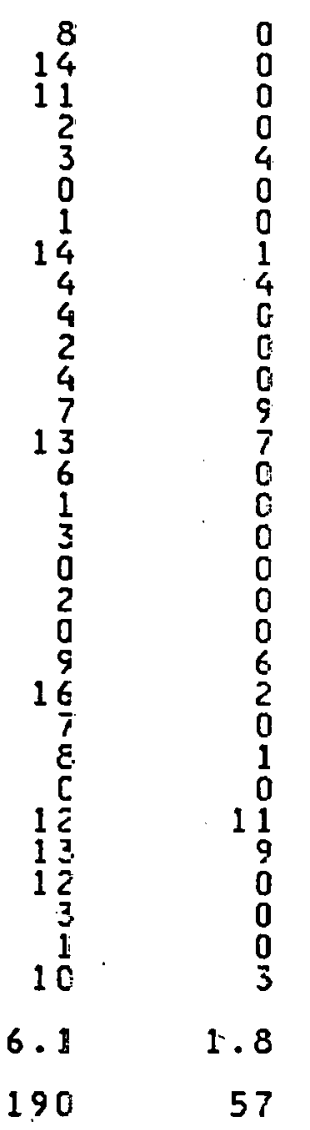

TOTAL
15.8

489

190

0
0
0
0
0
0
0
0
0
0
0
0
0
0
0
0
0
0
0
0
0
0
0
0
0
0
0
0
0
0
0
0
0
0
0

0
0
0
0
0
0
0
0
0
0
0
0
0
0
0
0
0
0
0
0
0
0
0
0
0
0
0
0
0
0
0

0
0
0

0
0
0
0
0
0
0
0
0
0
0
0
0
0
0
0
0
0
0
0
0
0
0
0
0
0
0
0
0
0
0
0
0

0
0
0
0
0
0
0
0
0
0
0
0
0
0
0
0
0
0
0
0
0
0
0
0
0
0
0
0
0
0
0
0
0
0
0

0

0

0
0
0

0

0.3

8 
ENVIRONMENTAL SUMMARY REPORT

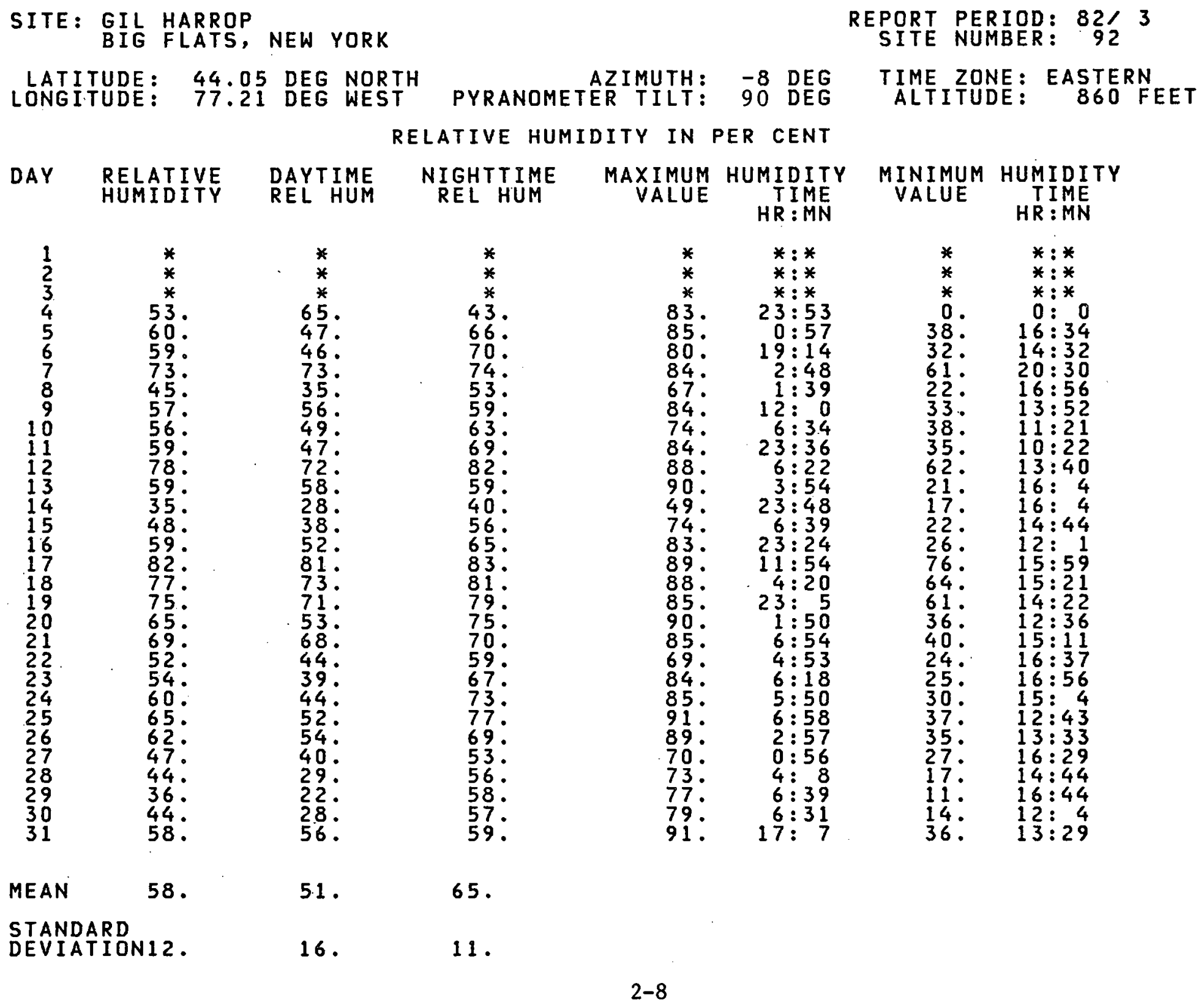




\begin{tabular}{|c|c|c|c|c|c|c|c|c|c|}
\hline ITE: $\begin{array}{r}\text { KALI } \\
\text { HEMP }\end{array}$ & $\begin{array}{l}\text { HOME } \\
\text { AD, N }\end{array}$ & NEW $Y$ & YORK & & & & $\begin{array}{l}\text { PORT PER } \\
\text { SITE NUME }\end{array}$ & $: \begin{array}{l}82 \\
: \quad 24\end{array}$ & 3 \\
\hline $\begin{array}{l}\text { LATITUDE: } \\
\text { LONGITUDE: }\end{array}$ & $\begin{array}{l}40.80 \\
73.40\end{array}$ & $\begin{array}{l}\text { DEG } \\
\text { DEG }\end{array}$ & $\begin{array}{l}\text { NORTH } \\
\text { WEST }\end{array}$ & PYRANOMETER & IMUTH: & $\begin{array}{ll}-6 & D E G \\
34 & D E G\end{array}$ & $\begin{array}{l}\text { TIME ZONE } \\
\text { ALT I TUDE : }\end{array}$ & $\begin{array}{l}\text { EAST } \\
200\end{array}$ & FEET \\
\hline & & & & INSOLATION & & & & & \\
\hline $\begin{array}{r}\text { INSO } \\
\text { BT }\end{array}$ & $\begin{array}{l}\text { OTAL } \\
\text { OLATION }\end{array}$ & $\begin{array}{r}\text { TOTA } \\
\text { INSD } \\
\text { BT }\end{array}$ & $\begin{array}{l}\text { AL DIFF } \\
\text { OLATION } \\
\text { TU /FT2 }\end{array}$ & $\begin{array}{l}\text { TOTAL DIR } \\
\text { INSDLATION } \\
\text { BTU } / F T 2\end{array}$ & $\begin{array}{r}\text { MAXIMU } \\
\text { INSO } \\
\text { VALU } \\
\text { BTU/HR }\end{array}$ & $\begin{array}{l}\text { M TOTAL } \\
\text { LATION } \\
\text { E TIME } \\
\text { FT2 HR:MN }\end{array}$ & $\begin{array}{r}\text { EXTRATERRES } \\
\text { INSOLATI } \\
\text { BTU } / F T\end{array}$ & ${ }_{2}^{T R}$ & \begin{tabular}{l} 
TTL INS \\
\hdashline EXT INS \\
PERCENT
\end{tabular} \\
\hline $\begin{array}{l}1 \\
2 \\
3 \\
4 \\
5 \\
6 \\
7 \\
8 \\
9 \\
10 \\
11 \\
12 \\
13 \\
14 \\
15 \\
16 \\
17 \\
18 \\
19 \\
20 \\
21 \\
22 \\
23 \\
24 \\
25 \\
26 \\
27 \\
28 \\
29 \\
30 \\
31\end{array}$ & 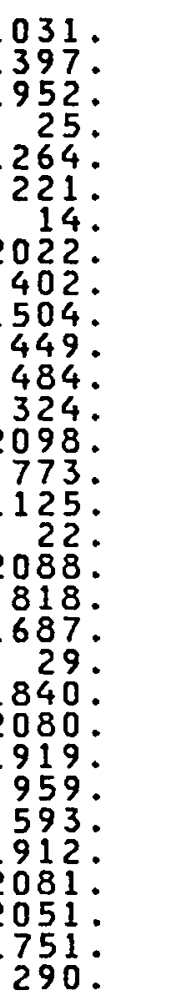 & & 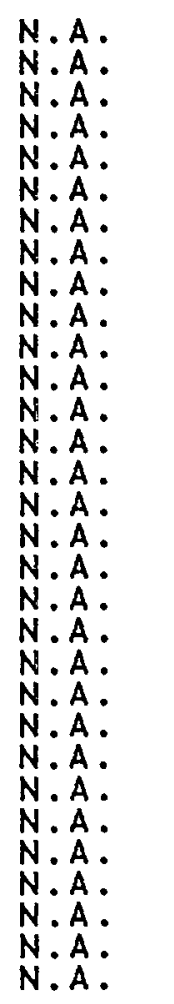 & 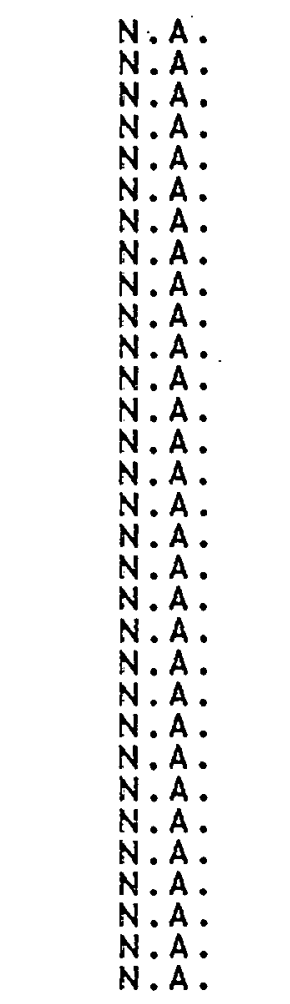 & $\begin{array}{r}232 \\
288 \\
304 \\
16 \\
291 \\
62 \\
110 \\
314 \\
206 \\
288 \\
167 \\
245 \\
88 \\
320 \\
222 \\
317 \\
23 \\
314 \\
242 \\
343 \\
20 \\
314 \\
314 \\
297 \\
301 \\
307 \\
343 \\
323 \\
310 \\
288 \\
154\end{array}$ & $\begin{array}{r}10: 30 \\
11: 45 \\
11: 21 \\
11: 42 \\
11: 46 \\
10: 42 \\
9: 48 \\
11: 12 \\
14: 32 \\
11: 19 \\
12: 6 \\
13: 10 \\
9: 52 \\
11: 20 \\
13: 50 \\
11: 37 \\
11: 29 \\
11: 44 \\
9: 59 \\
11: 53 \\
8: 39 \\
11: 00 \\
11: 25 \\
11: 59 \\
11: 53 \\
13: 12 \\
11: 46 \\
11: 22 \\
11: 32 \\
10: 40 \\
8: 16\end{array}$ & 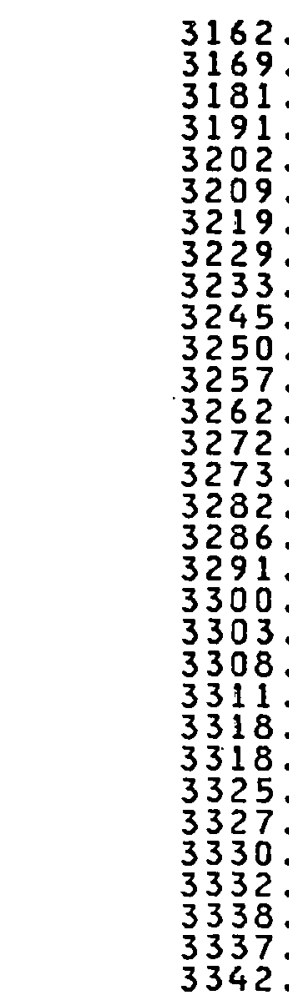 & & 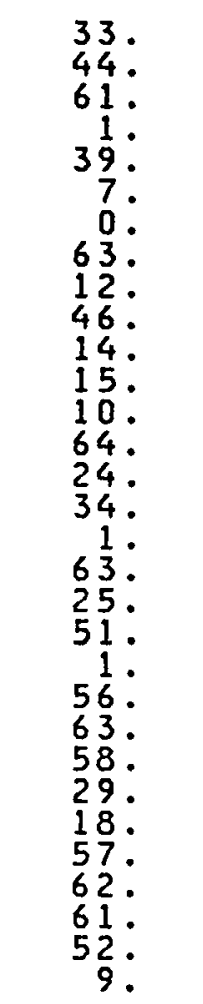 \\
\hline MEAN & 136 & & N.A. & N.A. & & & 3271 & & \\
\hline IATION & 68. & & N.A. & $N \cdot A$ & & & & & \\
\hline
\end{tabular}


ENVIRONMENTAL SUMMARY REPORT

SITE: KALIN HOME
HEMPSTEAD, NEW YORK

REPORT PERIOD: $82 / 3$

SITE NUMBER: 246

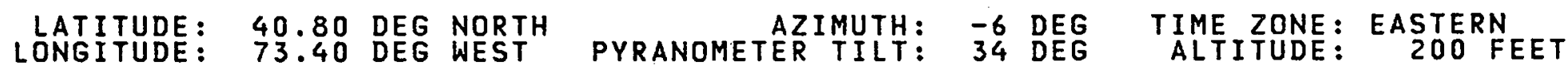
TEMPERATURES IN DEGREES F

\begin{tabular}{|c|c|c|c|c|c|c|}
\hline AVE & $\begin{array}{l}\text { RAGE } \\
\text { EMP }\end{array}$ & $\begin{array}{l}\text { DAYTIME } \\
\text { TEMP }\end{array}$ & $\begin{array}{c}\text { NIGHTTIME } \\
\text { TEMP }\end{array}$ & $\begin{array}{c}\text { MAX TEMPERATURE } \\
\text { VALUE TIIME } \\
\text { HR:MN }\end{array}$ & $\begin{array}{c}\text { MIN TEMPERATURE } \\
\text { VALUE TIME } \\
\text { HR:MN }\end{array}$ & $\begin{array}{l}\text { INLET WATER } \\
\text { TEMPERATURE }\end{array}$ \\
\hline $\begin{array}{l}1 \\
2 \\
3 \\
4 \\
5 \\
6 \\
7 \\
8 \\
9 \\
10 \\
11 \\
12 \\
13 \\
14 \\
15 \\
16 \\
17 \\
18 \\
19 \\
20 \\
21 \\
22 \\
23 \\
24 \\
25 \\
26 \\
27 \\
28 \\
29 \\
30 \\
31\end{array}$ & 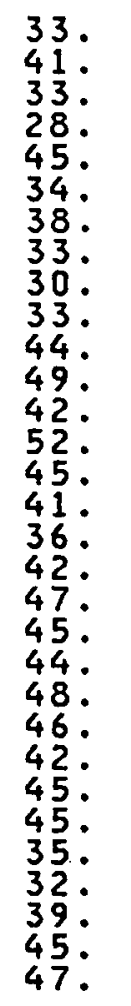 & 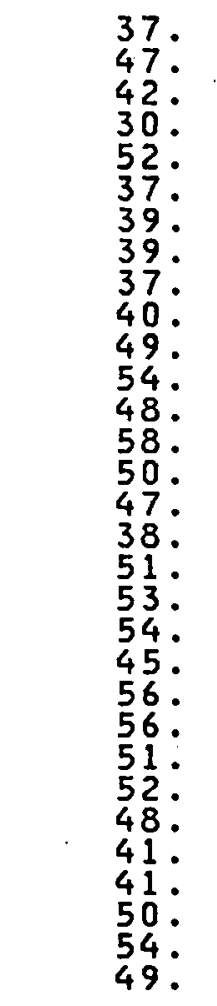 & 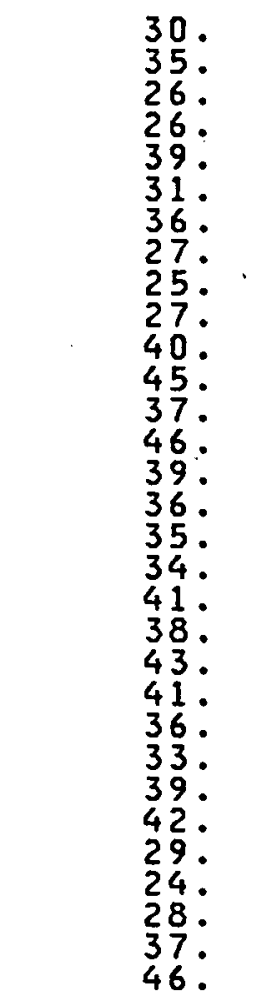 & 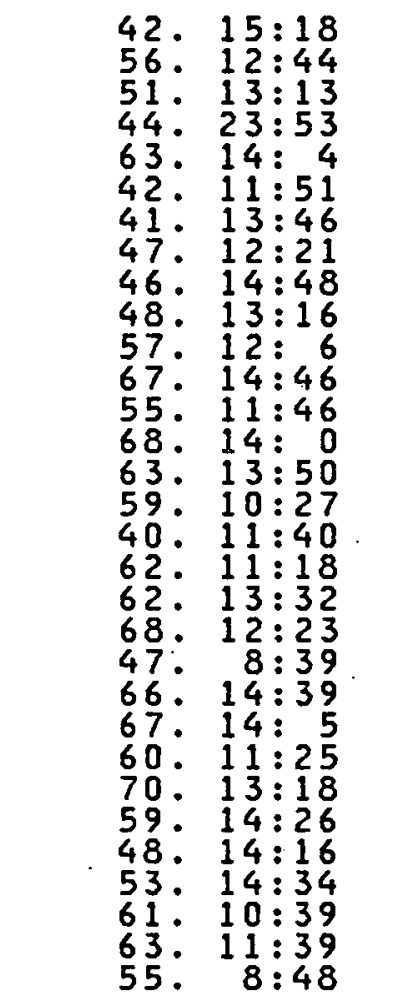 & 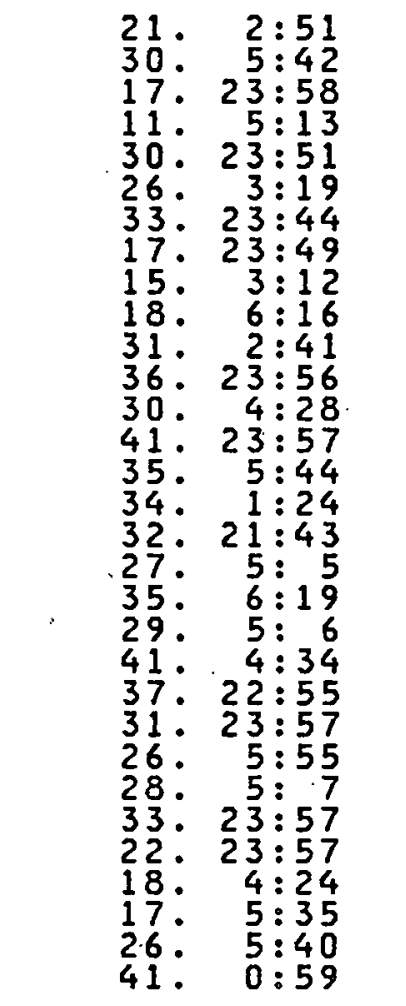 & 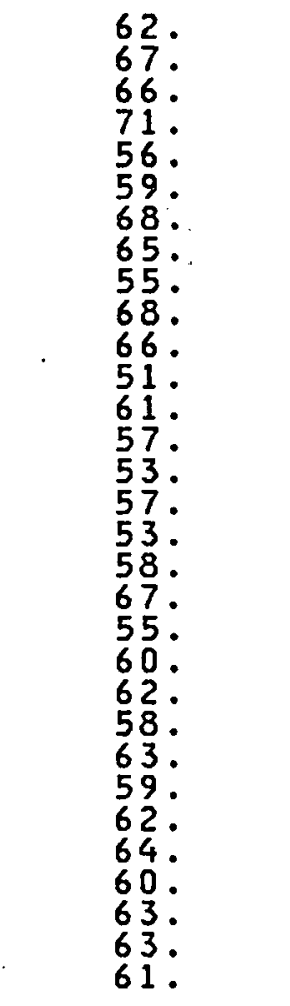 \\
\hline MEAN & 41. & 47. & 35. & & & 61. \\
\hline \multirow[t]{2}{*}{$\begin{array}{l}\text { STANDARD } \\
\text { DEVIATION }\end{array}$} & 6.3 & 7.2 & 6.5 & & & 5.0 \\
\hline & & & & $2-10$ & & \\
\hline
\end{tabular}


SITE : SOUTH BRIDGE TOWER

LATITUDE: $\quad 40.00$ DEG NORTH LONGITUDE: 74.00 DEG WEST
REPORT PERIOD: $82 / 3$

TIME ZONE: EASTERN

ALTITUDE : 137 FEET INSOLATION

DAY TOTAL
INSOLATION INSOL DIFF TOTAL DIR
BTUAFT2
BTU/FT2 INSOLATION
BTU/FTZ

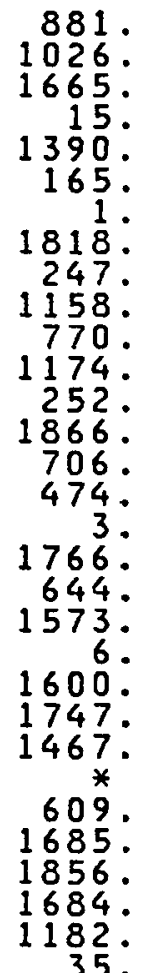

31

982 .

MEAN

STANDARD

DEVIATION 675.

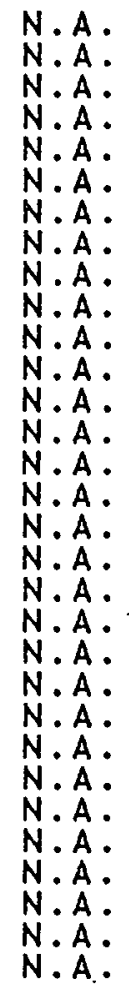

N.A.

N.A.

N.A.

N.A.

N.A.

N.A.

N.A.

N.A.

N.A.

N.A.

N.A.

N.A.

N.A.

N.A.

N.A.

N.A.

N.A.

$N: A$.

N.A.

N.A.

N.A.

N.A.

N.A.

N.A.

N.A.

N.A.

N.A.

N.A.

N.A.

N.A. $\begin{array}{cl}\text { XTRATERRESTRIAL } & \text { TTL INS } \\ \text { INSOLATION } & \text { EXT INS } \\ \text { BTUYFT2 } & \text { PXTERENT }\end{array}$ VALUE
BTU/HR/FT2 HR:MN

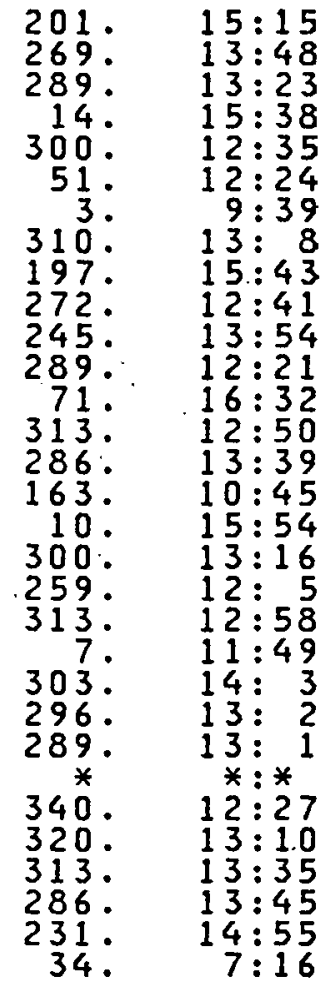

3224.

3234

3236.

3246 .

3247.

3256 .

$3257^{\circ}$.

3265 .

3265 .

3273 .

3273 .

3280.

3279 .

3285 .

3284.

3290.

3289.

3294 .

3292.

3291.

3203

3297 .

3294 .

$3297^{\circ}$.

32.94 .

.

3296.

3275 .

22.
27.

32 .

43 .

5 .

56.

35 .

36.

8.

57.

22 .

14.

54.

20.

$49^{\circ}$

45 .

18 .

51 .

56.

36 .

1 . 
ENVIRONMENTAL SUMMARY REPORT

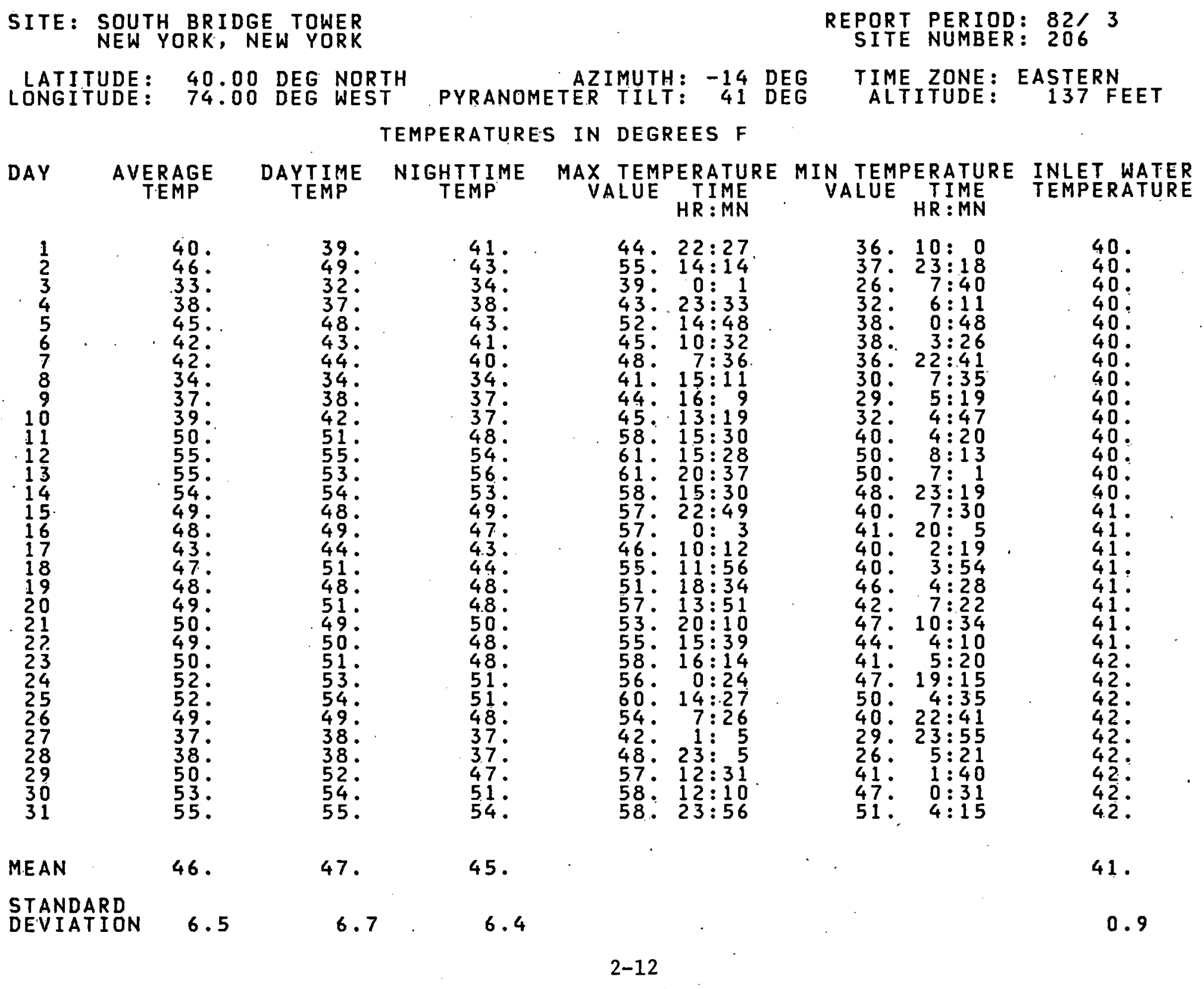


ENVIRONMENTAL SUMMARY REPORT

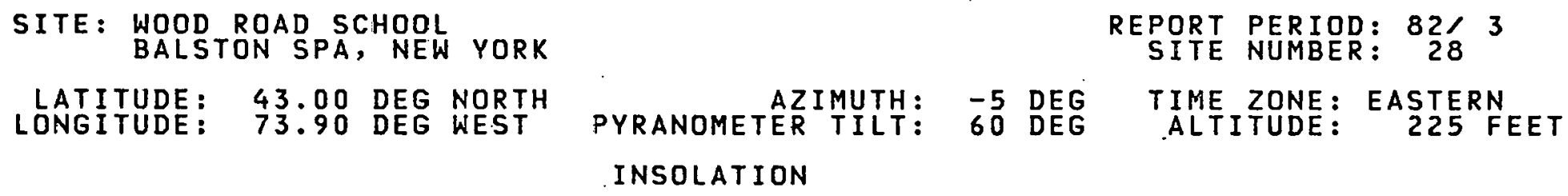

DAY

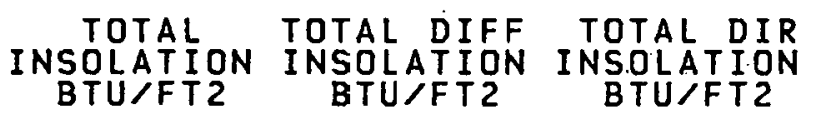

MAXIMUM TOTAL
EXTRATERRESTRIAL
INSOLATION
VALUE TNSOLATION
BTU/HR/FT2 HR:MN TTL I INS
EXT INS
PERCENT

783.

1434.

1434.

921 .

122 .

$885^{\circ}$

583.

1435.

$257^{\circ}$.

1931.

2218

209.

2256 .

425 .

$1929^{\circ}$

135.

2288 .

1762 .

1680.

1533.

2318 .

2145 .

1989.

N.A.

N.A.

N.A.

N.A.

N.A.

N.A:

N.A.

$N \cdot A$.

N.A.

N.A.

N.A.

N.A.

N.A.

$N \cdot A$.

N.A.

N.A.

N.A.

N.A.

N.A.

N.A.

N.A.

N.A.

N.A.

N.A.

N.A.

N.A.

N.A.

N.A.

N.A.

N.A.

N.A.

N.A.

N.A.

N:A:

N.A.

N.A.

N.A.

N.A.

N.A.

N.A.

N.A.

N.A.

N.A.

N.A.

N.A.

N.A.

N.A.

N.A.

N.A.

318. 11:30

$\begin{array}{rr}294 . & 10: 23 \\ 234 . & 9: 13\end{array}$

234. $9: 13$

$330 . \quad 12: 47$

168. $\quad 10: 1$

27. $11: 21$

75. $\quad 12: 49$

165. $10: 9$

309 . $11: 5$

243. 14:35

$362 . \quad 18: 6$

$339 . \quad 12: 13$

$336 . \quad 11: 15$

39. 15: 8

$330 . \quad 11: 53$

$213 . \quad 11: 7$

345. $12: 59$

$36 . \quad 9: 31$

$330 . \quad 11: 49$

321 . $11: 30$

312. $12: 2$

$324^{\circ} \quad 13: 21$

$297.12: 22$

333 . $11: 32$

324 .

306.

$11: 43$

N.A.

N.A.

3302 .

3299 .

3296 .

3296 .

$3291^{\circ}$

3290 .

3285

3274 .

3275 .

3265 .

3263.

3255 .

3237 .

3233 .

3220 .

3215 .

3195 .

3184.

3175 .

3164 .

3157 .

3146 .

3134 .

3126 .

3199 :

3090 :

3223.

68.

1236.

N.A.

N.A.
24.

23.

46 .

28 .

27.

18 :

44.

32 .

59 .

68.

$5 \frac{1}{6}$ :

$70^{\circ}$.

13.

60 .

$59^{4}$.

72.

56.

26.

$48^{\circ}$.

74 .

69.

15 .

MEAN 
ENVIRONMENTAL SUMMARY REPORT

SITE: WOOD ROAD SCHOOL YORK

REPORT PERIOD: $82 / 3$
SITE NUMBER: 28

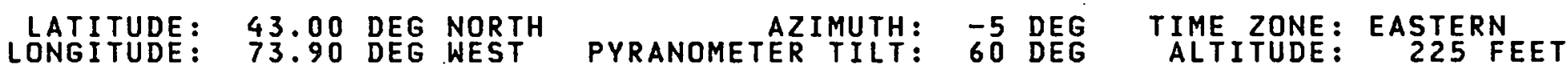

TEMPERATURES IN DEGREES F

\begin{tabular}{|c|c|c|c|c|c|}
\hline DAY & $\begin{array}{c}\text { AVERAGE } \\
\text { TEMP }\end{array}$ & $\begin{array}{c}\text { DAYT IME } \\
\text { TEMP }\end{array}$ & $\underset{\text { TEMP }}{\text { NIGHTTE }}$ & $\begin{array}{c}\text { MAX TEMPERATURE } \\
\text { VALUE TIME } \\
\text { HR:MN }\end{array}$ & $\begin{array}{c}\text { MIN TEMPERATURE INLET WATER } \\
\text { VALUE TIME } \\
\text { HR:MN }\end{array}$ \\
\hline
\end{tabular}

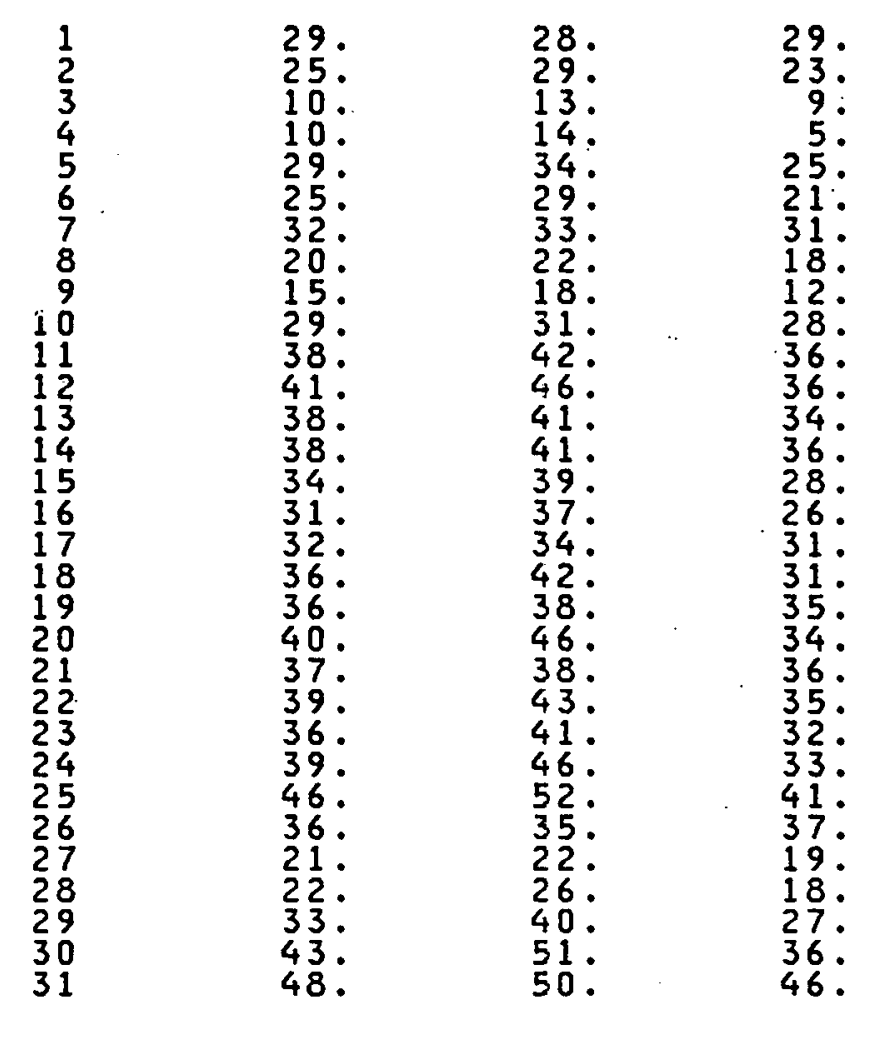

MEAN

32 .

35.

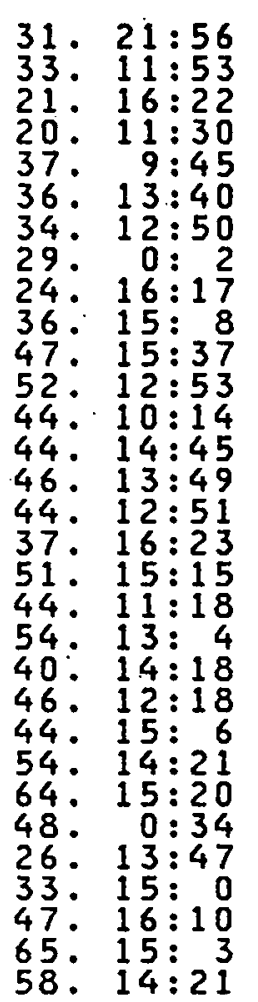

$21 \cdot 10: 58$

$15.23: 48$

$-13: 6: 8$

$17: 23: 59$

$10 \cdot \quad 3: 27$

29: $23: 51$

$4: 23: 53$

$21.6 \div 22$

$31 . \quad 0: 12$

34 . $3: 53$

$29: 21: 32$

$30.23: 38$

$22 \cdot 23: 57$

14. $5: 6$

22. $4: 23$

$33.6: 22$

$30.5: 47$

$32: 2: 3$

$30.22: 47$

$27.23: 59$

$19.6: 2$

$26.55: 49$

15. $22: 57$

7. $5: 58$

21: $\quad 5: 58$

$19: 5: 40$

40 : $2: 26$

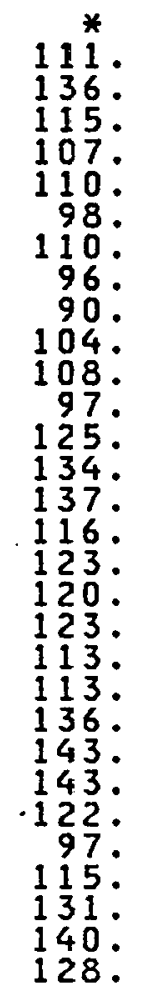

STANDARD

DEVIATION

9.7

10.3

29.

118

15.1 
ENVIRONMENTAL SUMMARY REPORT

SITE: WOOD ROAD SCHOOL YORK

REPORT PERIOD: $82 / 3$
SITE NUMBER: 28

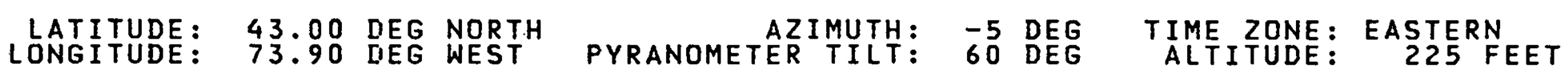
WIND: SPEED IN MPH-DIRECTION IN DEGREES

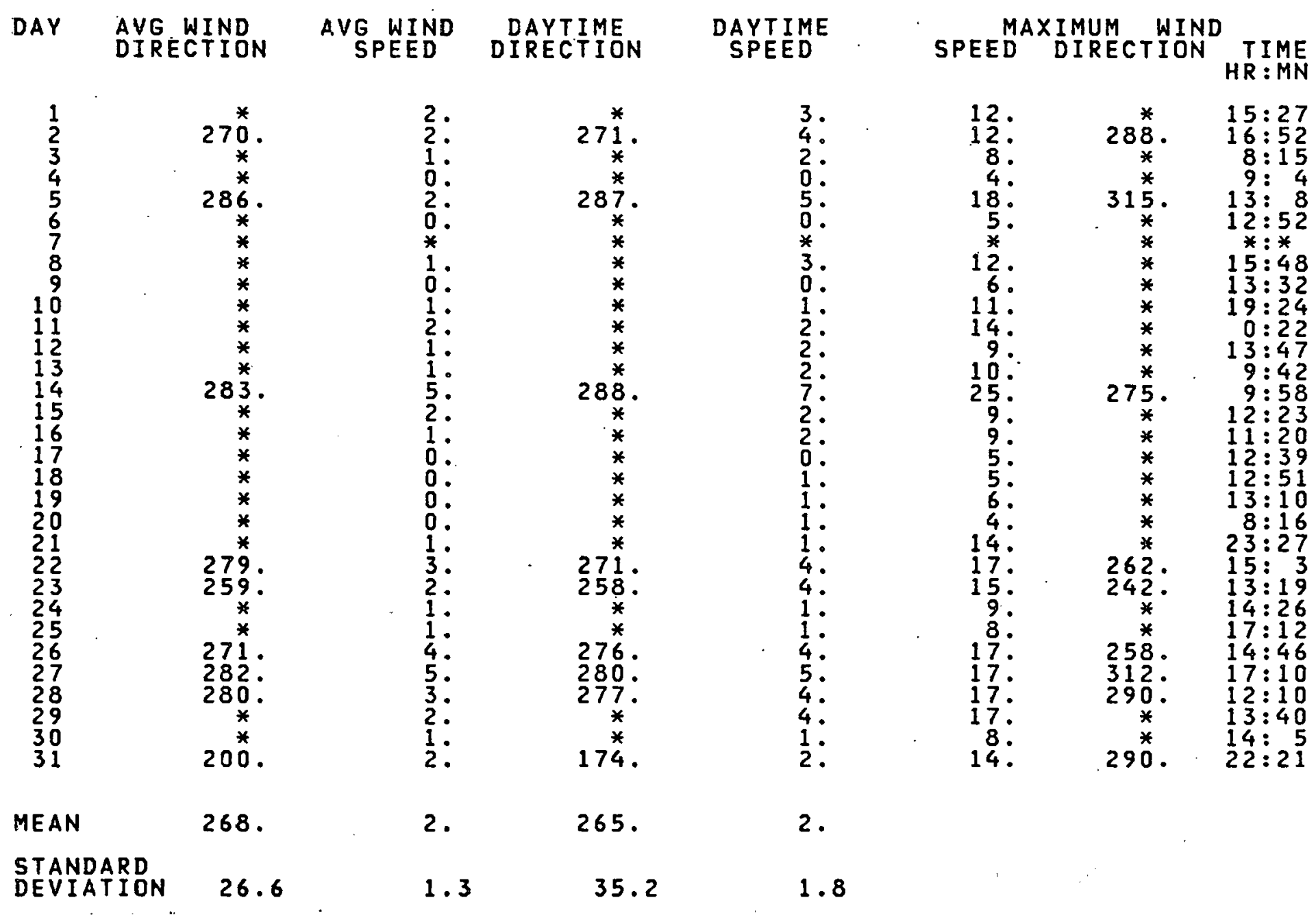


ENVIRONMENTAL SUMMARY REPORT

SITE: WOOD ROAD SCHOOL YORK

REPORT PERIOD: $82 / 3$
SITE NUMBER: 28

LATITUDE: 43.00 DEG NORTH AZIMUTH: -5 DEG TIME ZONE: EASTERN

LONGITUDE: 73.90 DEG WEST PYRANOMETER TILT: 60 DEG ALTITUDE: 225 FEET

WIND: NUMBER OF HOURS IN EACH VELOCITY INTERVAL

\begin{tabular}{|c|c|c|c|c|c|c|c|c|c|}
\hline DAY & $\begin{array}{l}0-3 \\
\text { MPH }\end{array}$ & $\begin{array}{l}4-7 \\
M P H\end{array}$ & $\begin{array}{l}8-12 \\
M P H^{2}\end{array}$ & $\begin{array}{l}13-18 \\
\mathrm{MPH} .\end{array}$ & $\underset{M P H}{19-24}$ & $\begin{array}{c}25-31 \\
\mathrm{MPH}\end{array}$ & $\begin{array}{c}32-38 \\
\mathrm{MPH}\end{array}$ & $\underset{38}{A B O V E}$ & $\begin{array}{c}\text { MISSING } \\
\text { HOURS }\end{array}$ \\
\hline $\begin{array}{l}1 \\
2 \\
3 \\
4 \\
5 \\
6 \\
77 \\
8 \\
9 \\
10 \\
11 \\
12 \\
13 \\
14 \\
15 \\
16 \\
17 \\
18 \\
19 \\
20 \\
21 \\
22 \\
23 \\
24 \\
25 \\
26 \\
27 \\
28 \\
29 \\
30 \\
31\end{array}$ & $\begin{array}{l}12 \\
199 \\
17 \\
24 \\
17 \\
24 \\
24 \\
21 \\
24 \\
24 \\
22 \\
23 \\
23 \\
99 \\
22 \\
23 \\
24 \\
24 \\
24 \\
24 \\
22 \\
14 \\
20 \\
24 \\
23 \\
11 \\
17 \\
19 \\
24 \\
20\end{array}$ & $\begin{array}{r}1 \\
2 \\
1 \\
0 \\
6 \\
0 \\
0 \\
3 \\
0 \\
0 \\
2 \\
1 \\
1 \\
12 \\
2 \\
1 \\
0 \\
0 \\
0 \\
0 \\
2 \\
10 \\
4 \\
0 \\
1 \\
13 \\
17 \\
7 \\
5 \\
0 \\
0 \\
3\end{array}$ & $\begin{array}{l}0 \\
0 \\
0 \\
0 \\
1 \\
0 \\
0 \\
0 \\
0 \\
0 \\
0 \\
0 \\
0 \\
3 \\
0 \\
0 \\
0 \\
0 \\
0 \\
0 \\
0 \\
0 \\
0 \\
0 \\
0 \\
0 \\
0 \\
0 \\
0 \\
0 \\
0\end{array}$ & $\begin{array}{l}0 \\
0 \\
0 \\
0 \\
0 \\
0 \\
0 \\
0 \\
0 \\
0 \\
0 \\
0 \\
0 \\
0 \\
0 \\
0 \\
0 \\
0 \\
0 \\
0 \\
0 \\
0 \\
0 \\
0 \\
0 \\
0 \\
0 \\
0 \\
0 \\
0 \\
0\end{array}$ & $\begin{array}{l}0 \\
0 \\
0 \\
0 \\
0 \\
0 \\
0 \\
0 \\
0 \\
0 \\
0 \\
0 \\
0 \\
0 \\
0 \\
0 \\
0 \\
0 \\
0 \\
0 \\
0 \\
0 \\
0 \\
0 \\
0 \\
0 \\
0 \\
0 \\
0 \\
0 \\
0\end{array}$ & $\begin{array}{l}0 \\
0 \\
0 \\
0 \\
0 \\
0 \\
0 \\
0 \\
0 \\
0 \\
0 \\
0 \\
0 \\
0 \\
0 \\
0 \\
0 \\
0 \\
0 \\
0 \\
0 \\
0 \\
0 \\
0 \\
0 \\
0 \\
0 \\
0 \\
0 \\
0 \\
0 \\
0\end{array}$ & $\begin{array}{l}0 \\
0 \\
0 \\
0 \\
0 \\
0 \\
0 \\
0 \\
0 \\
0 \\
0 \\
0 \\
0 \\
0 \\
0 \\
0 \\
0 \\
0 \\
0 \\
0 \\
0 \\
0 \\
0 \\
0 \\
0 \\
0 \\
0 \\
0 \\
0 \\
0 \\
0\end{array}$ & $\begin{array}{l}0 \\
0 \\
0 \\
0 \\
0 \\
0 \\
0 \\
0 \\
0 \\
0 \\
0 \\
0 \\
0 \\
0 \\
0 \\
0 \\
0 \\
0 \\
0 \\
0 \\
0 \\
0 \\
0 \\
0 \\
0 \\
0 \\
0 \\
0 \\
0 \\
0 \\
0 \\
0\end{array}$ & $\begin{array}{r}11 \\
3 \\
6 \\
0 \\
0 \\
0 \\
0 \\
0 \\
0 \\
0 \\
0 \\
0 \\
0 \\
0 \\
0 \\
0 \\
0 \\
0 \\
0 \\
0 \\
0 \\
0 \\
0 \\
0 \\
0 \\
0 \\
0 \\
0 \\
0 \\
0 \\
1\end{array}$ \\
\hline AVERAGE & 20.2 & 3.0 & 0.1 & 0.0 & 0.0 & 0.0 & 0.0 & 0.0 & 0.7 \\
\hline TOTAL & 625 & 94 & 4 & 0 & 0 & 0. & 0 & 0 & 21 \\
\hline
\end{tabular}


ZONE 3

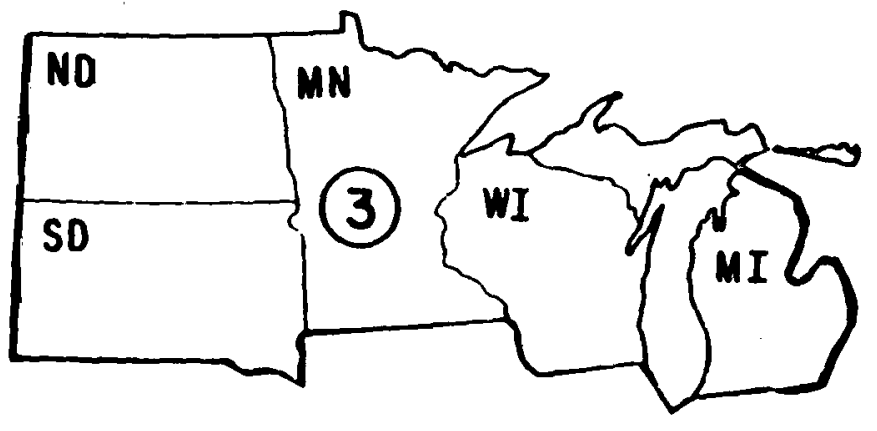


SITE: ARNO KAHN

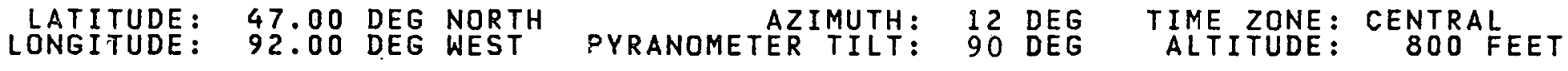
INSOLATION

\begin{tabular}{|c|c|c|c|c|c|c|c|}
\hline IN & $\begin{array}{l}\text { TOTAL } \\
\text { OLATION } \\
\text { TU/FT2 }\end{array}$ & $\begin{array}{l}\text { TOTAL DIFF } \\
\text { INSOLATION } \\
\text { BTU/FT2 }\end{array}$ & $\begin{array}{l}\text { TOTAL DIR } \\
\text { INSOLATION } \\
\text { BTU/FT2 }\end{array}$ & $\begin{array}{r}\text { MAXIMUM T } \\
\text { INSOLAT } \\
\text { VALUE } \\
\text { BTU/HR/FT2 }\end{array}$ & $\begin{array}{l}\text { OTAL } \\
\text { ION } \\
\text { TIME } \\
\text { HR:MN }\end{array}$ & $\begin{array}{l}\text { EXTRATERRESTRIAL } \\
\text { INSOLATION } \\
\text { BTU/FT2 }\end{array}$ & $\begin{array}{l}\text { TTL I VS } \\
\frac{\text { EXT I VS }}{\text { PERCENT }}\end{array}$ \\
\hline $\begin{array}{r}1 \\
2 \\
3 \\
4 \\
5 \\
6 \\
7 \\
8 \\
9 \\
10 \\
11 \\
12 \\
13 \\
14 \\
15 \\
16 \\
17 \\
18 \\
199 \\
20 \\
21 \\
22 \\
23 \\
24 \\
25 \\
26 \\
27 \\
28 \\
29 \\
30 \\
31\end{array}$ & 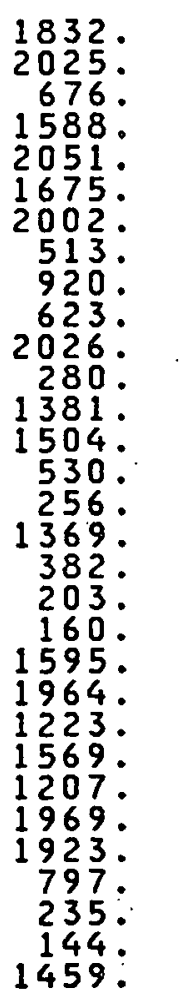 & 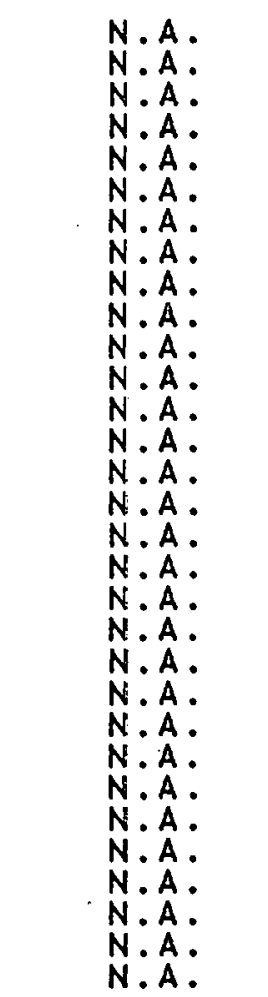 & 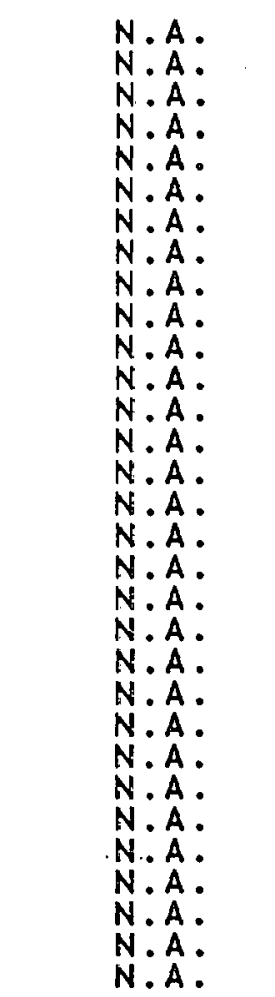 & 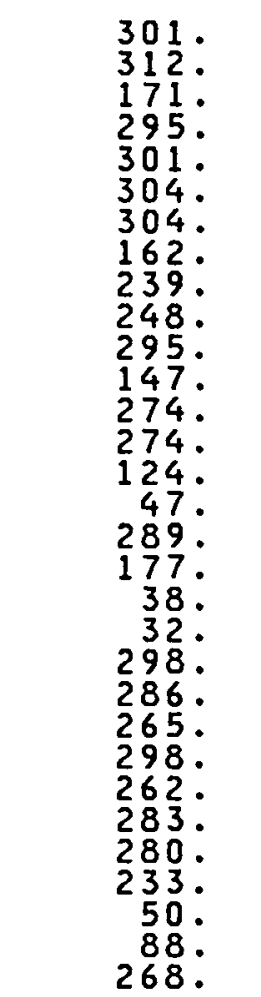 & 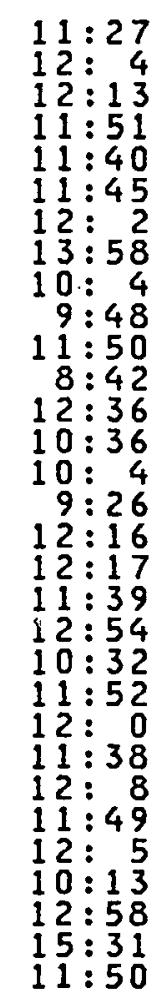 & 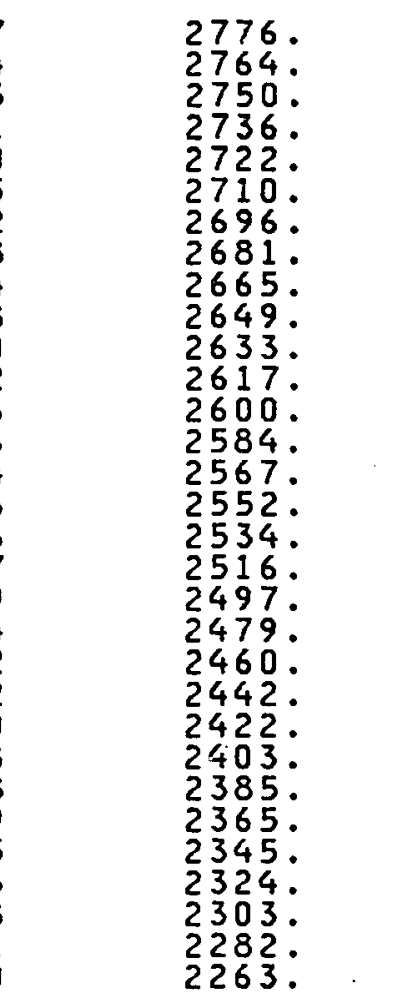 & 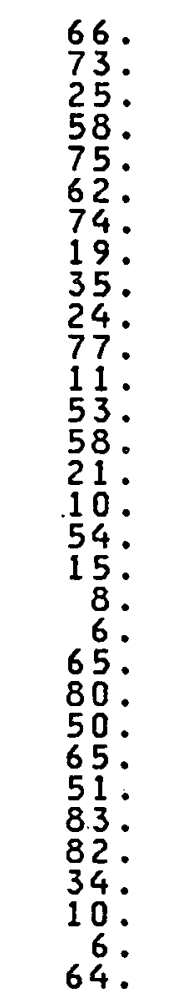 \\
\hline EAN & 1164. & N.A. & N.A. & & & 2539 . & \\
\hline $\begin{array}{l}\text { ANDARD } \\
\text { VIATION }\end{array}$ & 68 & N.A. & N.A. & & & 157. & \\
\hline
\end{tabular}


ENVIRONMENTAL SUMMARY REPORT

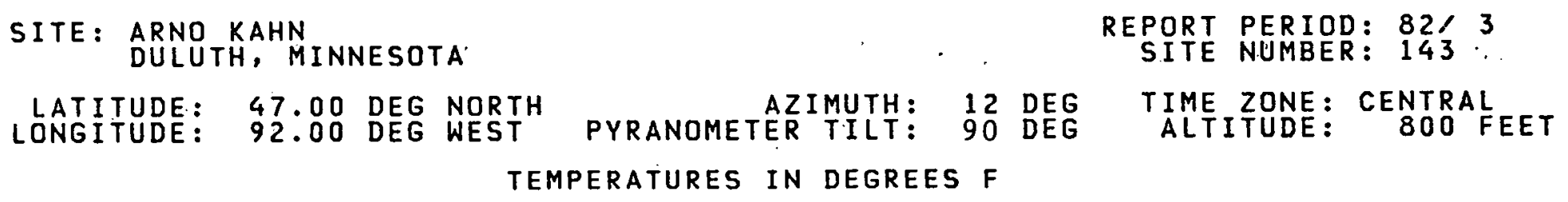

35. $22: 7$

21. $14: 34$

34. $12: 3$

$29 \cdot 14: 41$

$34.12: 54$

38. $8: 33$

24. $15: 35$

43 . $22: 31$

$60: 23: 38$

63. $13: 19$

$47 \cdot 13: 38$

$61 \cdot 23: 8$

$46.14: 14$

44: 11 : 2

$37.11: 32$

34. $9: 26$

$49 \cdot 15: 57$

$53.14: 22$

$38.13: 30$

$34.13: 39$

36 . $18: 56$

$48.9: 41$

60:18:29

61. $11: 33$

$48 \cdot 15: 37$

MEAN

29.

32.

27.

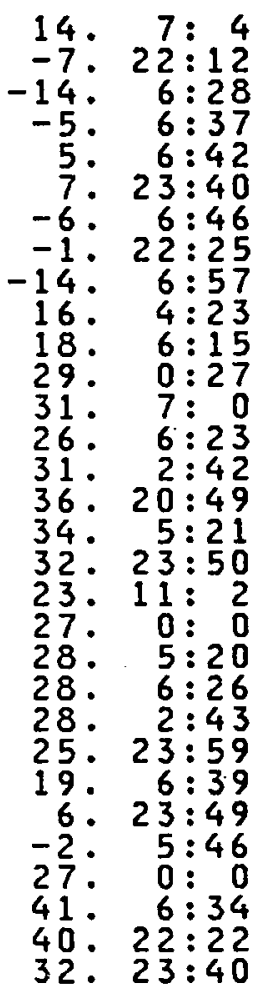

53.

53.

51.

49.

48.

47.

49.

$51^{\circ}$.

49

STANDARD

DEVIATION

11.4

10.3

12.5

49.

50.

50 .

49.

51.

48.

48 .

51 .

52 .

50 .

48.

47.

52.

51.

50 .

50. 
SITE : ARNO KAHN DULUTH, MINNESOTA

REPORT PERIOD: $82 / 3$
SITE NUMBER: 143

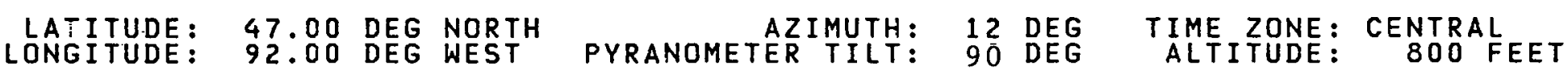
WIND: SPEED IN MPH-DIRECTION IN DEGREES

DAY AVG WIND
DIRECTION

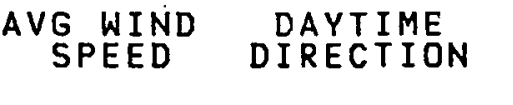

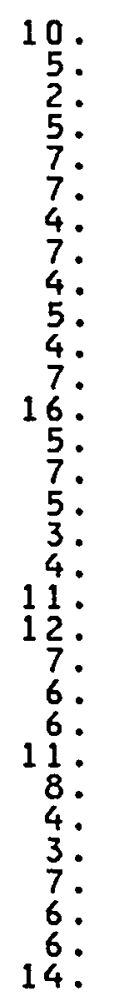

7.

3.2
DAYTIME
SPEED

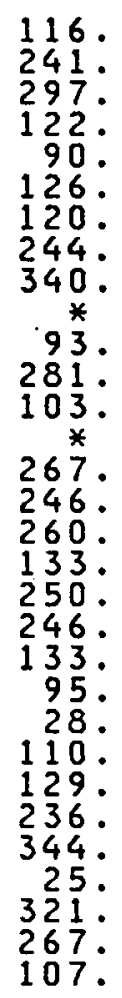

185.

94.3

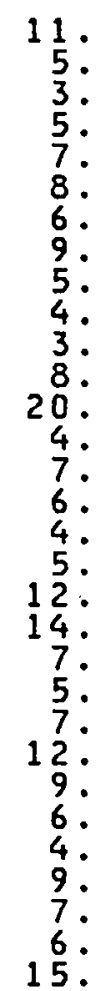

8.

3.9
SPEED MAXIMUM WIND ${ }_{\text {DIRECTION TIME }}^{\text {TIRN }}$

\begin{tabular}{|c|c|c|}
\hline 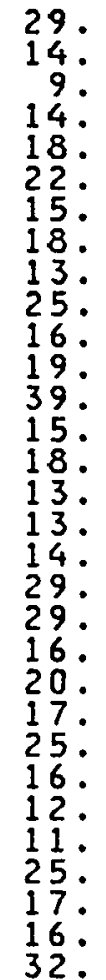 & $\begin{array}{r}124 . \\
166 . \\
312 \\
142 \\
70 \\
135 \\
137 \\
255 \\
322 \\
3 \\
1\end{array}$ & $\begin{array}{r}8: 58 \\
0: 31 \\
8: 56 \\
16: 50 \\
13: 10 \\
12: 38 \\
0: 44 \\
11: 50 \\
16: 12 \\
23: 40 \\
0: 44 \\
14: 50 \\
11: 54 \\
0: 4 \\
21: 43 \\
8: 11 \\
10: 35 \\
14:: 4 \\
23: 23 \\
8: 54 \\
20: 40 \\
3: 30 \\
17: 47 \\
8: 42 \\
4: 26 \\
14: 45 \\
13: 14 \\
11: 17 \\
15: 56 \\
1: 52 \\
3: 58\end{array}$ \\
\hline
\end{tabular}




\section{ENVIRONMENTAL SUMMARY REPORT}

SITE : ARNO KAHN

REPORT PERIOD: $82 / 3$
SITE NUMBER: 143

$\begin{array}{llll}\text { LATITUDE: } & 47.00 \\ \text { LONGITUDE: } & 92.00 & \text { DEG NORTH WEST PYRANOMETER TILT: } & 12 \text { DEG DEG TIME ZONE: CENTRAL }\end{array}$ WIND: NUMBER OF HOURS IN EACH VELOCITY INTERVAL

\begin{tabular}{|c|c|c|c|c|c|c|c|c|c|}
\hline DAY & $\begin{array}{l}0-3 \\
M P H\end{array}$ & $\begin{array}{l}4-7 \\
M P H\end{array}$ & $\begin{array}{l}8-12 \\
M P H\end{array}$ & $\begin{array}{l}13-18 \\
M P H\end{array}$ & $\begin{array}{c}19-24 \\
\mathrm{MPH}\end{array}$ & $\begin{array}{l}25-31 \\
\mathrm{MPH}\end{array}$ & $\begin{array}{l}32-38 \\
M P H\end{array}$ & $\begin{array}{c}\text { ABOVE } \\
38\end{array}$ & $\begin{array}{c}\text { MISSING } \\
\text { HOURS }\end{array}$ \\
\hline $\begin{array}{r}1 \\
2 \\
3 \\
4 \\
5 \\
6 \\
7 \\
8 \\
9 \\
10 \\
11 \\
12 \\
13 \\
14 \\
15 \\
16 \\
17 \\
18 \\
19 \\
20 \\
21 \\
22 \\
23 \\
24 \\
25 \\
26 \\
27 \\
28 \\
29 \\
30 \\
31\end{array}$ & $\begin{array}{r}0 \\
6 \\
21 \\
10 \\
1 \\
4 \\
10 \\
6 \\
8 \\
8 \\
13 \\
18 \\
3 \\
0 \\
8 \\
0 \\
6 \\
14 \\
11 \\
2 \\
0 \\
0 \\
4 \\
4 \\
2 \\
0 \\
0 \\
8 \\
18 \\
1 \\
0 \\
2 \\
2\end{array}$ & $\begin{array}{r}6 \\
18 \\
3 \\
10 \\
21 \\
8 \\
12 \\
99 \\
16 \\
7 \\
5 \\
1.2 \\
0 \\
16 \\
17 \\
18 \\
10 \\
12 \\
1 \\
15 \\
16 \\
16 \\
18 \\
4 \\
9 \\
15 \\
6 \\
13 \\
21 \\
18 \\
1\end{array}$ & $\begin{array}{r}15 \\
0 \\
0 \\
4 \\
2 \\
12 \\
2 \\
9 \\
0 \\
2 \\
1 \\
1 \\
9 \\
8 \\
0 \\
7 \\
0 \\
0 \\
1 \\
11 \\
11 \\
8 \\
3 \\
4 \\
15 \\
15 \\
1 \\
1 \\
10 \\
3 \\
3 \\
4 \\
2\end{array}$ & $\begin{array}{r}3 \\
0 \\
0 \\
0 \\
0 \\
0 \\
0 \\
0 \\
0 \\
2 \\
0 \\
0 \\
9 \\
0 \\
0 \\
0 \\
0 \\
0 \\
10 \\
6 \\
0 \\
1 \\
0 \\
5 \\
0 \\
0 \\
0 \\
0 \\
0 \\
0 \\
20\end{array}$ & $\begin{array}{l}0 \\
0 \\
0 \\
0 \\
0 \\
0 \\
0 \\
0 \\
0 \\
0 \\
0 \\
0 \\
6 \\
0 \\
0 \\
0 \\
0 \\
0 \\
0 \\
2 \\
0 \\
0 \\
0 \\
0 \\
0 \\
0 \\
0 \\
0 \\
0 \\
0 \\
0\end{array}$ & $\begin{array}{l}0 \\
0 \\
0 \\
0 \\
0 \\
0 \\
0 \\
0 \\
0 \\
0 \\
0 \\
0 \\
1 \\
0 \\
0 \\
0 \\
0 \\
0 \\
0 \\
0 \\
0 \\
0 \\
0 \\
0 \\
0 \\
0 \\
0 \\
0 \\
0 \\
0 \\
0\end{array}$ & $\begin{array}{l}0 \\
0 \\
0 \\
0 \\
0 \\
0 \\
0 \\
0 \\
0 \\
0 \\
0 \\
0 \\
0 \\
0 \\
0 \\
0 \\
0 \\
0 \\
0 \\
0 \\
0 \\
0 \\
0 \\
0 \\
0 \\
0 \\
0 \\
0 \\
0 \\
0 \\
0\end{array}$ & $\begin{array}{l}0 \\
0 \\
0 \\
0 \\
0 \\
0 \\
0 \\
0 \\
0 \\
0 \\
0 \\
0 \\
0 \\
0 \\
0 \\
0 \\
0 \\
0 \\
0 \\
0 \\
0 \\
0 \\
0 \\
0 \\
0 \\
0 \\
0 \\
0 \\
0 \\
0 \\
0\end{array}$ & $\begin{array}{l}0 \\
0 \\
0 \\
0 \\
0 \\
0 \\
0 \\
0 \\
0 \\
0 \\
0 \\
0 \\
0 \\
0 \\
0 \\
0 \\
0 \\
0 \\
0 \\
0 \\
0 \\
0 \\
0 \\
0 \\
0 \\
0 \\
0 \\
0 \\
0 \\
0 \\
1\end{array}$ \\
\hline AVERAGE & 5.7 & 11.1 & 5.1 & 1.8 & 0.3 & 0.0 & 0.0 & 0.0 & 0.0 \\
\hline TOTAL & 176 & 343 & 159 & 56 & 8 & 1 & 0 & 0 & 1 \\
\hline
\end{tabular}


ENVIRONMENTAL SUMMARY REPORT

SITE: ARNO KAHN

REPORT PERIOD: $82 / 3$

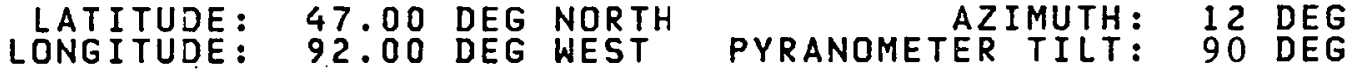

TIME ZONE: CENTRAL

RELATIVE HUMIDITY IN PER CENT

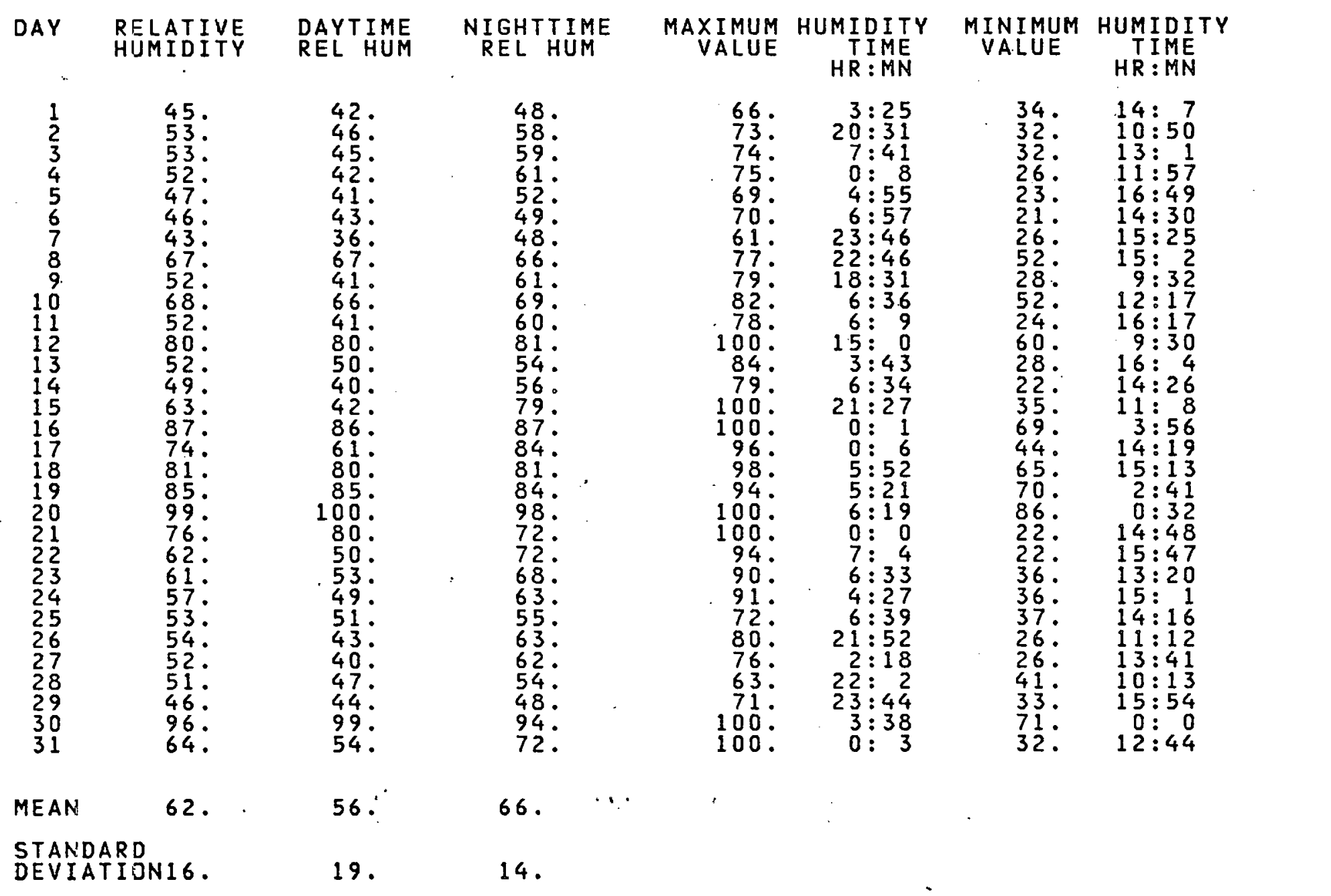


SITE : EROS DATA CENTER

SIOUX FALLS, SOUTH DAKOTA
REPORT PERIOD: $82 / 3$
SITE NUMBER: 247

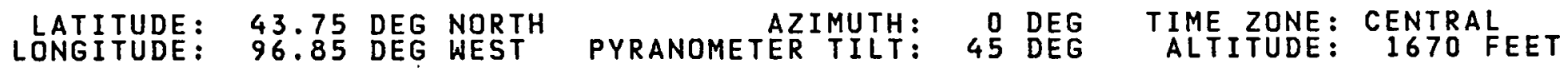
INSOLATION
DAY

$$
\begin{aligned}
& \text { TOTAL TOTAL DIFF TOTAL DIR } \\
& \text { INSOLATION INSOLATION INSOLATION } \\
& \text { BTUFTZ BTUTFT }
\end{aligned}
$$

\section{INSOLATION}

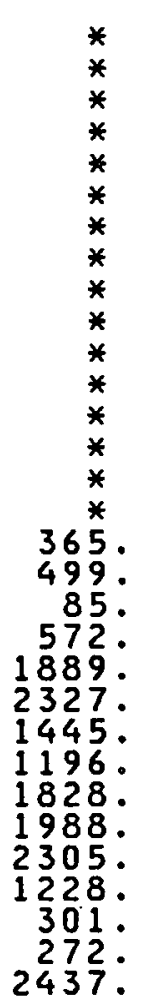

MEAN $1249 . *$

STANDARD DEVIATION

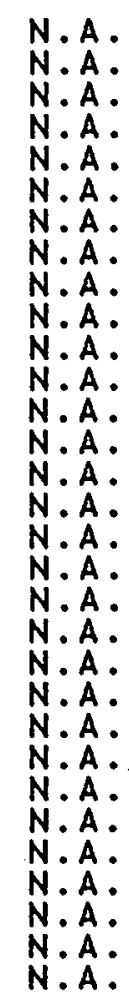

N.A.

N.A.

\begin{abstract}
N.A.
N.A.

N.A:

N.A.

N.A.

N.A.

N.A.

N.A.

N.A:

N.A.

N.A.

N.A.

N.A.

N.A.

N.A.

N.A.

N.A.

N.A.

N.A.

N.A:

N.A.

N.A.

N.A.

N.A.

N.A.

N.A.

N.A.
\end{abstract}

N.A.

N.A.
MAXIMUM TOTAL INSOLATION BTU/HR/FT2 HR:MN

\begin{tabular}{|c|c|}
\hline * & $\|^{*}: *$ \\
\hline * & *:* \\
\hline * & $*_{*}^{*} *$ \\
\hline * & $*: *$ \\
\hline * & *:* \\
\hline * & $*: *$ \\
\hline * & $*: *$ \\
\hline * & $* * *$ \\
\hline * & $*:$ * \\
\hline * & $*: *$ \\
\hline * & *:* \\
\hline * & *:* \\
\hline * & $*: *$ \\
\hline * & $*: *$ \\
\hline * & $*: *$ \\
\hline * & * : * \\
\hline $\begin{array}{r}666^{\circ} \\
264^{\circ}\end{array}$ & $\begin{array}{l}11 \\
13: 18\end{array}$ \\
\hline $\begin{array}{r}26 \\
142 .\end{array}$ & $\begin{array}{r}8: 19 \\
10: 54\end{array}$ \\
\hline 3 & $\frac{1}{2}: 59$ \\
\hline 3. & $11: 20$ \\
\hline & $\begin{array}{l}10: 54 \\
12: 20\end{array}$ \\
\hline 33 & $\begin{array}{l}12: 32 \\
12: 27\end{array}$ \\
\hline & $12 \vdots 13$ \\
\hline & $\begin{array}{r}8 \vdots 3 \\
12 \vdots 21\end{array}$ \\
\hline
\end{tabular}

INSOTATSTRIA BTU/FT?

TTL INS
EXT INS
PERCENT
3271. 3276. 3289 . 3297 . 3308 . 3311. 3318 . 3321. 3327. 3334 . 3336 . 3341 . 3342 . 3346 . 3346 . $3350^{\circ}$ 3352 . $3351^{\circ}$. 3348 . 3345 . 3342 . 3338 . 3331 . 3327 . 3323 . 3319 .

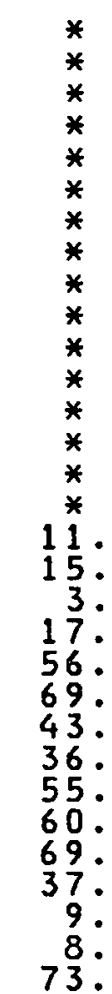

3325 .

23. 
SITE: EROS DATA CENTER
SIOUX FALLS, SOUTH DAKOTA

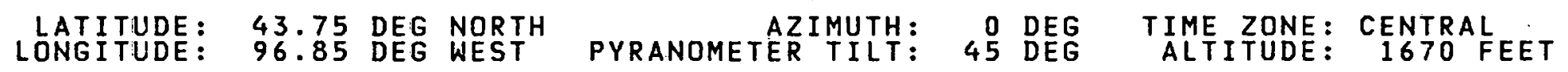
TEMPERATURES IN DEGREES F

\begin{tabular}{|c|c|c|c|c|c|c|}
\hline DAY & $\begin{array}{c}\text { AVERAGE } \\
\text { TEMP }\end{array}$ & $\begin{array}{l}\text { DAYTIME } \\
\text { TEMP }\end{array}$ & NIGHTTIME & $\begin{array}{c}\text { MAX TEMPERATURE } \\
\text { VALUE TIME } \\
\text { HR:MN }\end{array}$ & $\begin{array}{c}\text { MIN TEMPERATURE } \\
\text { VALUE TIME } \\
\text { HR:MN }\end{array}$ & $\begin{array}{l}\text { INLET WATER } \\
\text { TEMPERATURE }\end{array}$ \\
\hline
\end{tabular}
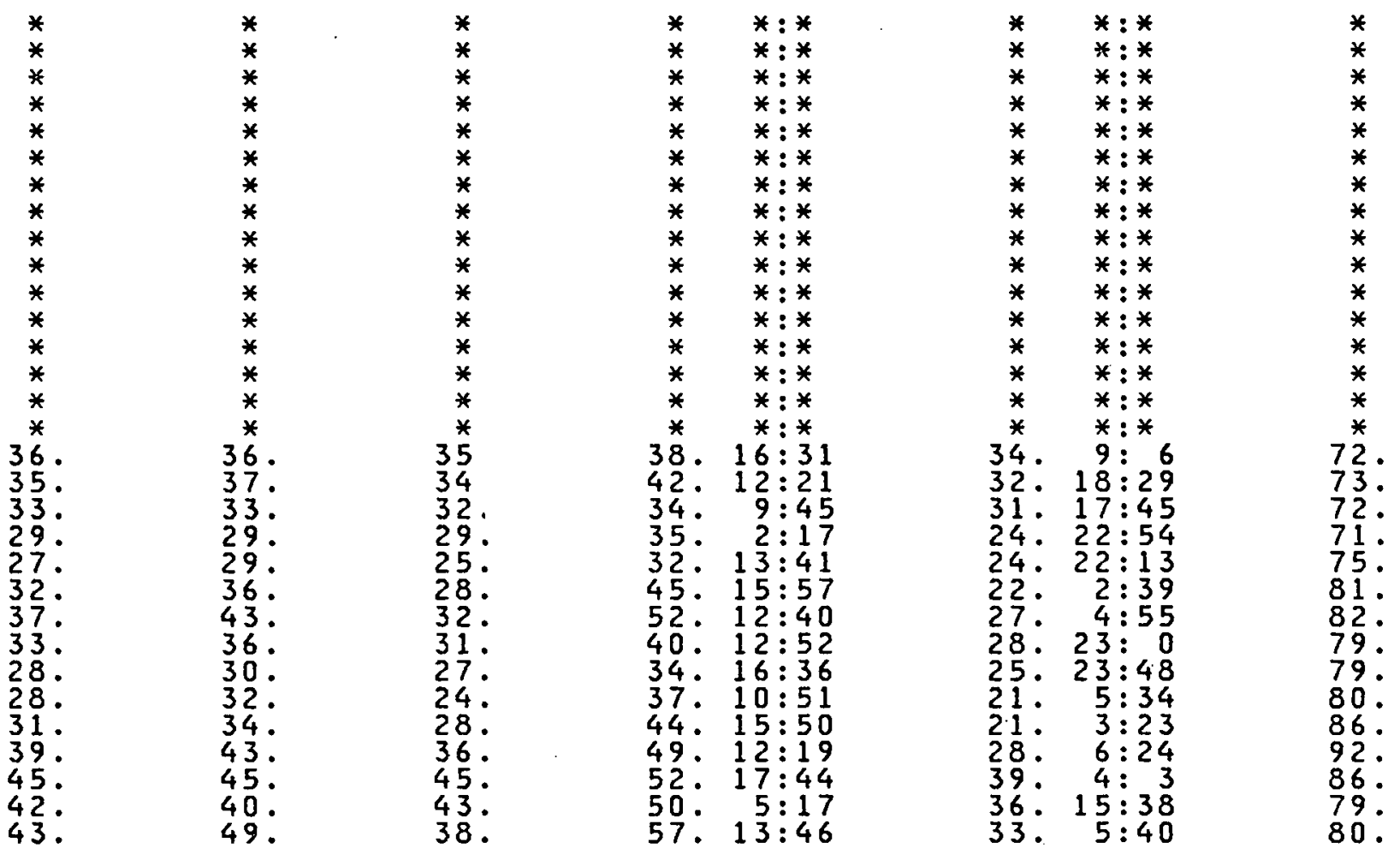

MEAN $35 .^{*}$ $37 .^{*}$ $33 .^{*}$ 79. STANDARD 5.7

6.0

6.1 5.9 
ENVIRONMENTAL SUMMARY REPORT

SITE: ISAKSON HOME
ANOKA, MINNESOTA

LATITUDE: $\quad 45.15$ DEG NORTH LONGITUDE: 93.45 DEG WEST
REPORT PERIOD: $82 / 3$
SITE NUMBER: 242

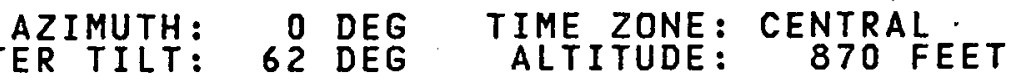

\section{INSOLATION}

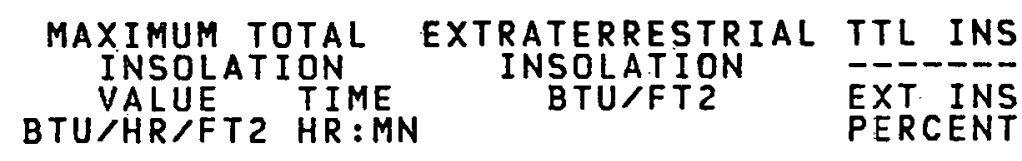

$$
\begin{aligned}
& \text { INSOLATION } \\
& \text { VALUE TTIME } \\
& \text { BTU/HR /FT2 HR:MN }
\end{aligned}
$$

INSOLATION BTULFT2

DAY $\begin{array}{ccc}\text { TOTAL } & \text { TOTAL DIFF } & \text { TOTAL DIR } \\ \text { INSOLATION } & \text { INSOLATION } & \text { INSOLATION } \\ \text { BTU/FT2 } & \text { BTU/FT2 } & \text { BTU/FT2 }\end{array}$

269. 10:8

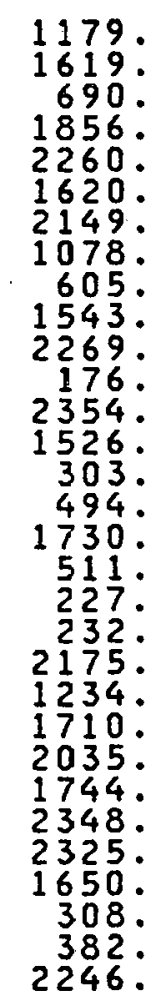

1373.

N.A.

N.A.

N.A.

N.A.

N.A.

N.A.

N.A.

N.A.

N.A.

N.A.

N.A.

N.A.

N.A.

N.A.

N.A.

N.A.

N.A.

N.A.

N.A.

N.A.

N.A.:

N.A.

N.A.

N.A.

N.A.

N.A.

N.A.

N.A.

N.A.

N.A.

N.A.

N.A.

N.A.

N.A.

N.A.

N.A.

N.A.

N.A.

N.A.

N.A:

N.A.

N.A.

N.A.

N.A.

N.A.

N.A.

N.A.

N.A.

N.A.

N.A.

N.A.

N.A.

N.A.

325. $11: 1$

$\frac{181}{350} \quad 12: 51$

$350 . \quad 12: 29$

$362.12: 13$

$3500^{\circ}: 12: 37$

$331 . \quad 12: 42$

109. 16:58

$350 . \quad 11: 33$

350 . $\quad 12: 18$

$35 . \quad \quad 9: 8$

253. $12: 20$

56.

103.

337.

$129^{\circ}$.

$41^{\circ}$.

356.

347.

353.

343 .

340 .

343 .

284.

$331^{\circ}$

N.A.

$12: 14$

$14: 20$

$13: 21$

$11: 32$

10.41

$12: 46$

$11: 26$

11: 157

$12: 1$

$12: 6$

$12: 14$

$11: 58$

$13: 14$

$12: 32$

$10: 46$
$12: 40$

MEAN

N.A.

N.A.

STANDARD
DEVIATION

769.
3299

3295 .

3296 .

3293 .

3293 .

$3291^{\circ}$

3284 .

3285 .

$3280^{\circ}$

3274 .

3265 .

3265.

3254 .

3246.

3238.

3232.

$3217^{\circ}$.

3208 .

3194

3182.

3156.

3145 .

3130.

3115.

3103.

3091 .

3231.

$65 . .2$

N.A.

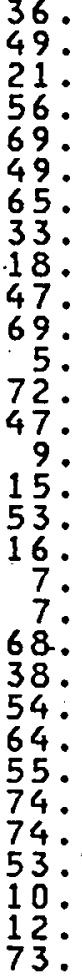


ENVIRONMENTAL SUMMARY REPORT

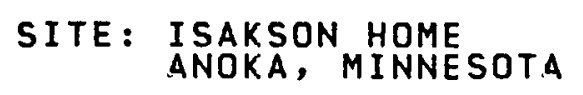

REPORT PERIOD: $82 / 3$
SITE NUMBER: 242

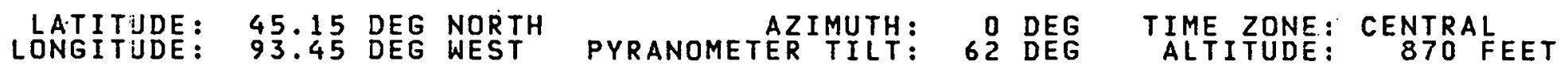

TEMPERATURES IN DEGREES $F$

DAY AVERAGE DAYTIME NIGHTTIME MAX TEMPERATURE MIN TEMPERATURE INLET WATER
TEMP
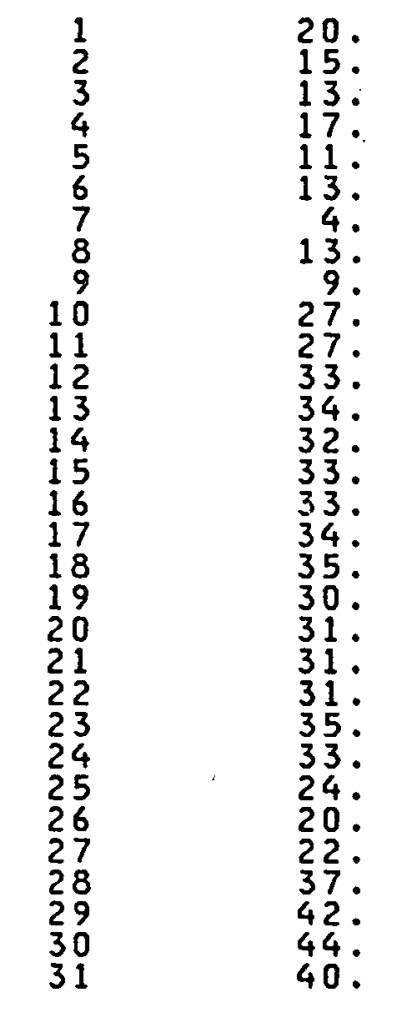

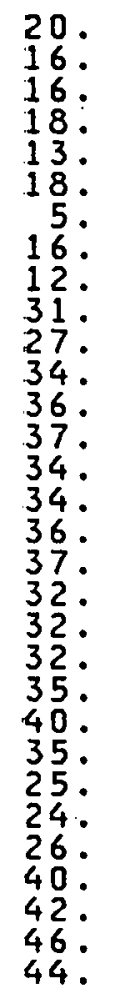

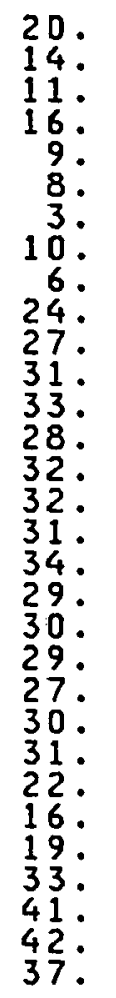

$26 \cdot 15: 28$

$23: 14: 18$

$26.14: 30$

$23.12: 50$

13 . $13: 15$

$21 \cdot 9: 52$

$20 \cdot 15: 17$

$42 \cdot 14: 40$

$32 \cdot 15: 14$

39 . $15: 5$

45. $13: 51$

$37 \cdot 14: 32$

$37.15: 29$

44. $12: 54$

$41: 14: 39$

$35 \cdot 14: 44$

$37 \cdot 15: 38$

40 : $12: 18$

47. $14: 15$

39 . $12: 33$

$28.16: 27$

$30.14: 6$

$34.15: 10$

$46.15: 22$

48. $12: 48$

$50: 14: 43$

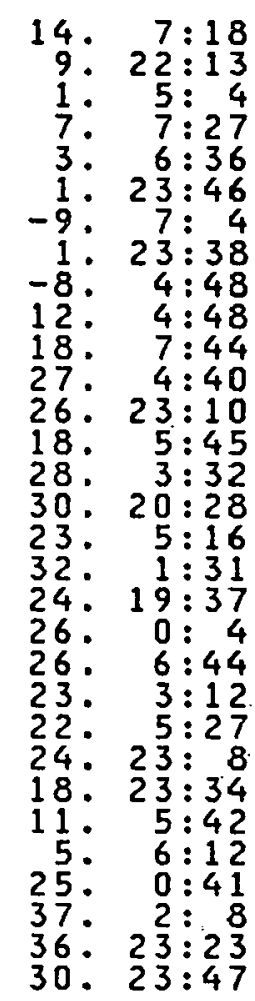

55 .

57 .

54.

MEAN

27.

29.

24.

58

58 .

58 .

STANDARD

DEVIATION 10.5

10.5

10.6

57.

1.5 
ZONE 4

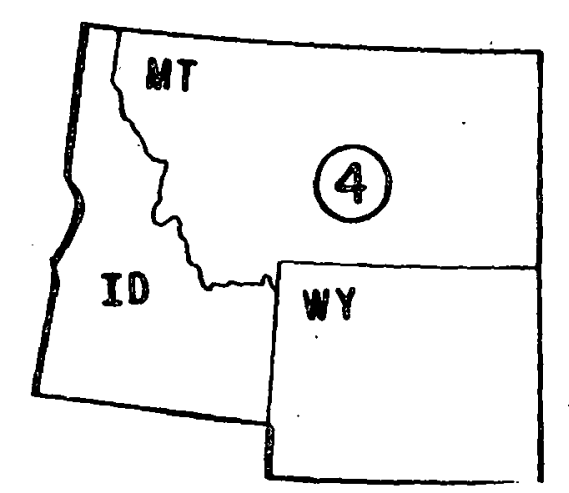


NO VALID DATA 
2ONE 5

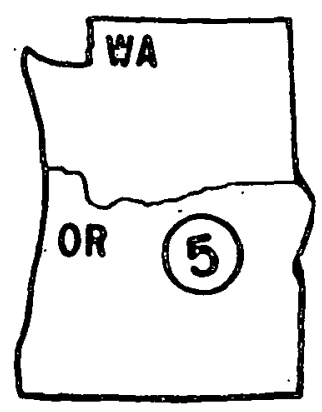


No VALID DATA

5-1 
ZONE 6

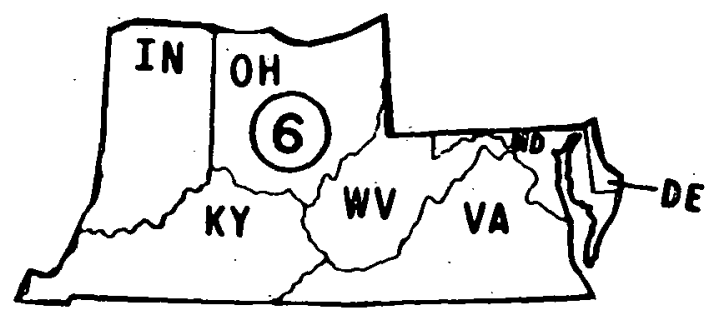


ENVIRONMENTAL SUMMARY REPORT

SITE: CARTER COUNTY GRAYSON, KENTUCKY

REPORT PERIOD: $82 / 3$
SITE NUMBER: 244

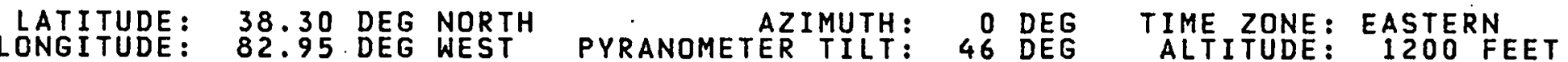
INSOLATION

DAY $\begin{array}{ccc}\text { TOTAL } & \text { TOTAL DIFF } & \text { TOTAL DIR } \\ \text { INSOLATION INSOLATION } & \text { INSOLATION } \\ \text { BTU/FT2 } & \text { BTU/FT2 } & \text { BTU/FT2 }\end{array}$

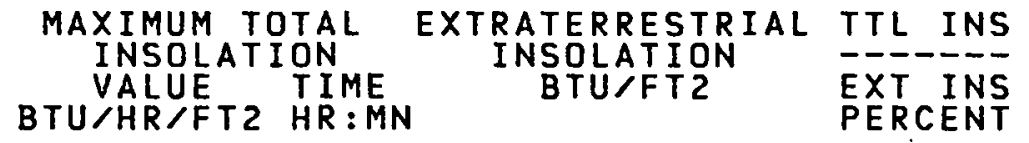

N.A.

N.A.

N.A.

N.A.

N.A.

$N \cdot A$.

N.A.

1019.

110 .

2176 .

N.A.

N.A.

N.A.

N.A.

N.A.

957 .

563.

1252 .

1609

1015 .

171.

2185 .

2059 .

296.

$1976^{\circ}$

821.

2095.

1994 .

617
1402

$1418^{\circ}$.

2325.

2325.

1482 .

N.A.

N.A.

N.A.

N.A.

N.A.

N.A.

N.A.

N.A.

N.A.

N.A.

$N \cdot A$.

N.A.

N.A.

N.A.

N.A.

N.A:

N.A:

N.A.

N.A.

N.A.

N.A.

N.A.

N.A.

N.A.

$N \cdot A$.

$N \cdot A$.

$N \cdot A$.

N.A.

N.A.

N.A.

N.A.

N.A.

N.A.

N.A.

N.A.

N.A.

N.A.

354. $12: 10$

$12: 35$

216. $11: 4$

288. $\quad 11: 38$

$11: 49$

$\begin{array}{ll}55 & 11: 49 \\ 42 & 8: 37\end{array}$

94. $15: 37$

357. $12: 8$

$321^{*} . \quad 11^{*}:{ }^{*} 33$

$246^{\circ}-19: 58$

$324 . \quad 11: 22$

$335 . \quad 12: 21$

280. $13: 21$

$14: 28$

$163 . \quad 15: 49$

$321 . \quad 12: 8$

$13: 32$

$12: 55$

$12: 55$

$12: 24$

$13: 5$

$11: 52$

$13: 55$

$12: 10$

$11: 6$

$12: 8$

$12: 24$

$12: 43$

MEAN

1177 .

N.A.

N.A.

3340 .

3344 .

3343

3346 .

3343 .

3345 .

$3349^{\circ}$.

3350 .

3346 .

3347 .

3347

3347 .

3343 .

3341 .

3336 .

3335 .

3333 .

3330
3328

3322 .

3314 .

3306 .

3293

3285.

3280 .

3271 .

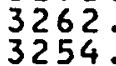

56.

327.

324 .

27.

30 .

$8:$

3
8
6

2 *

29.

37 .

48 .

38.

13 .

13
65
62

62 .

$21^{\circ}$.

25.

60

19.

43.

43 .

70 .

45
30

\section{STANDARD}

743

N.A.

N.A.

3325 .

29. 
ENVIRONMENTAL SUMMARY REPORT

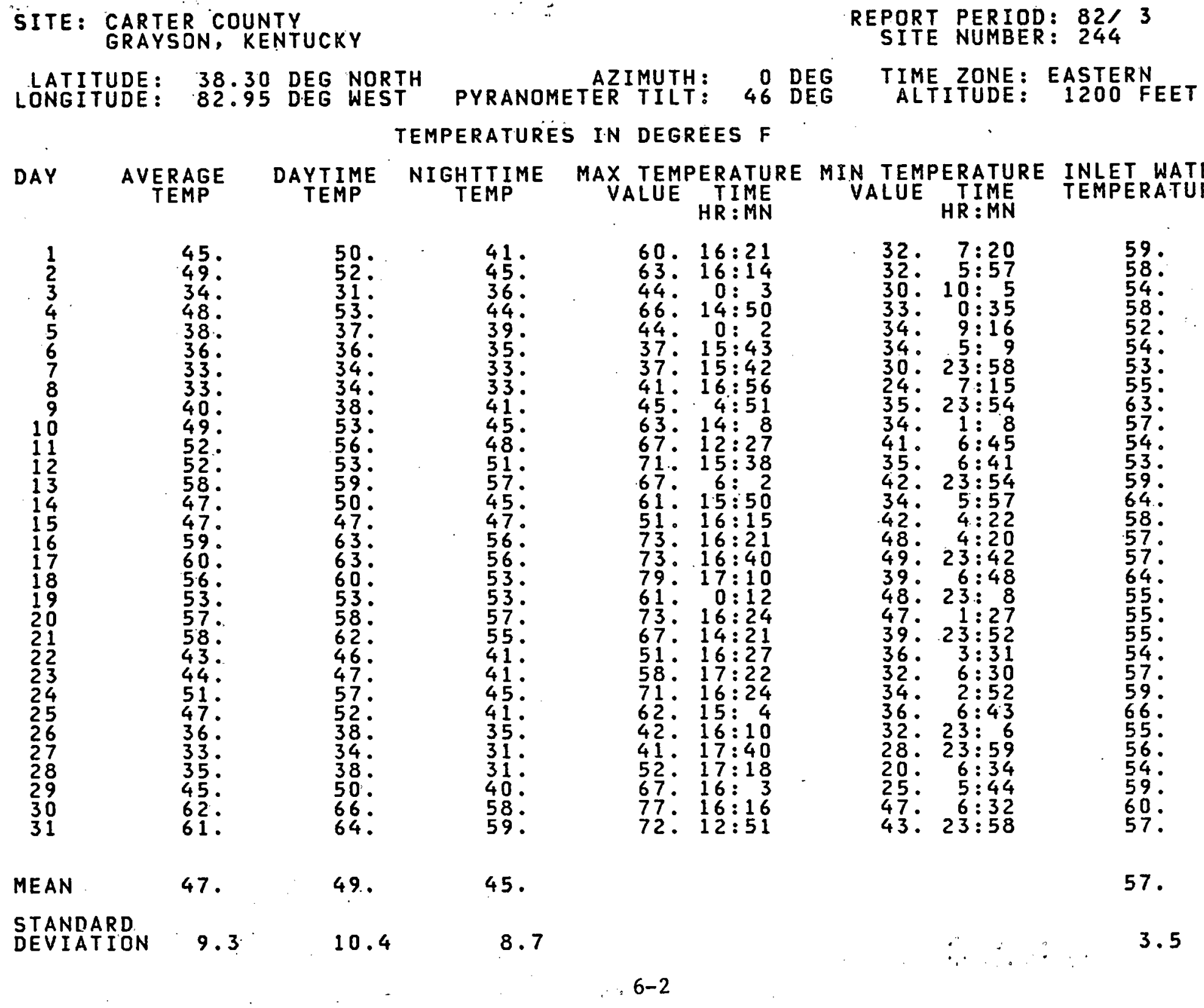




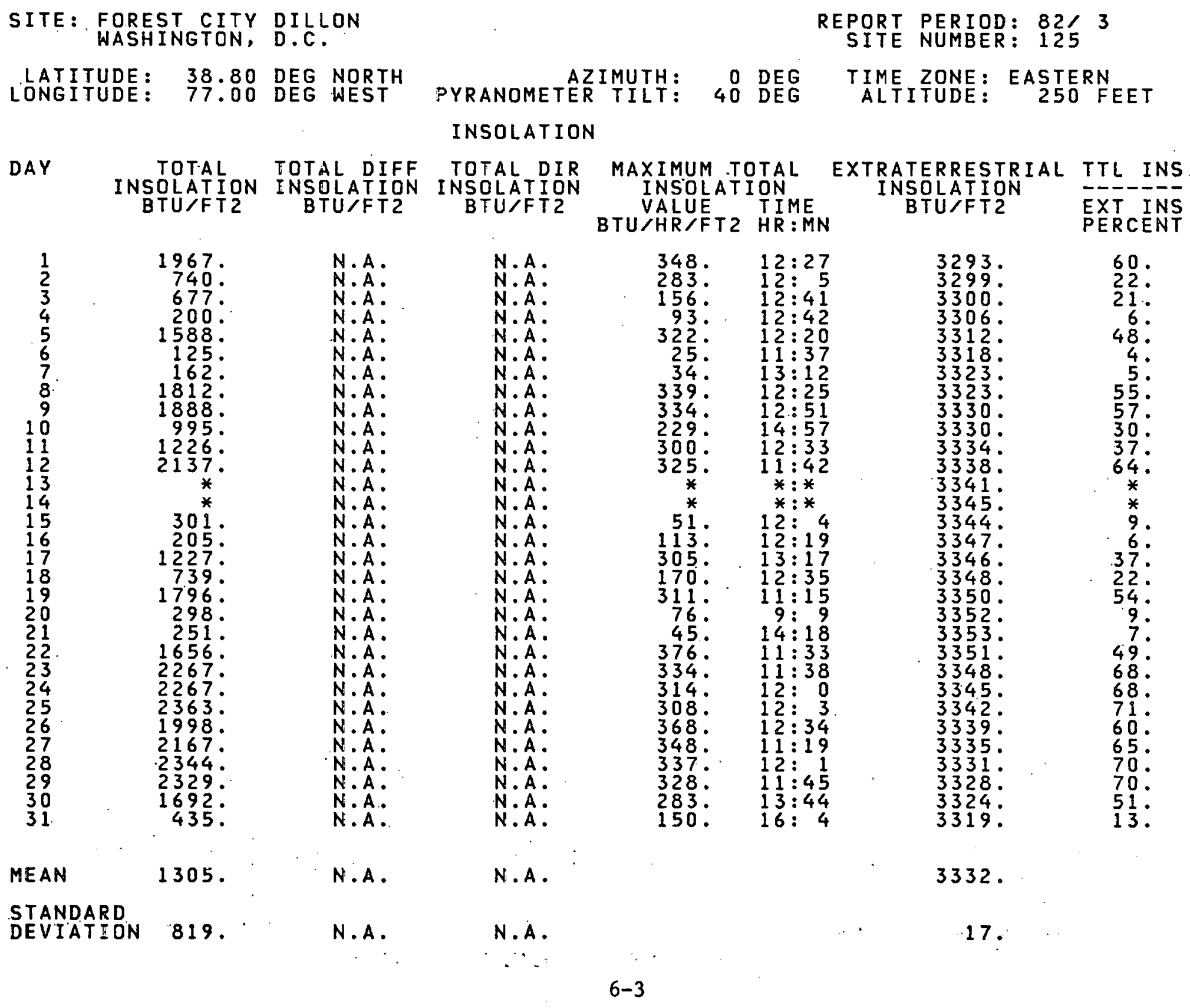


ENVIRONMENTAL SUMMARY REPORT

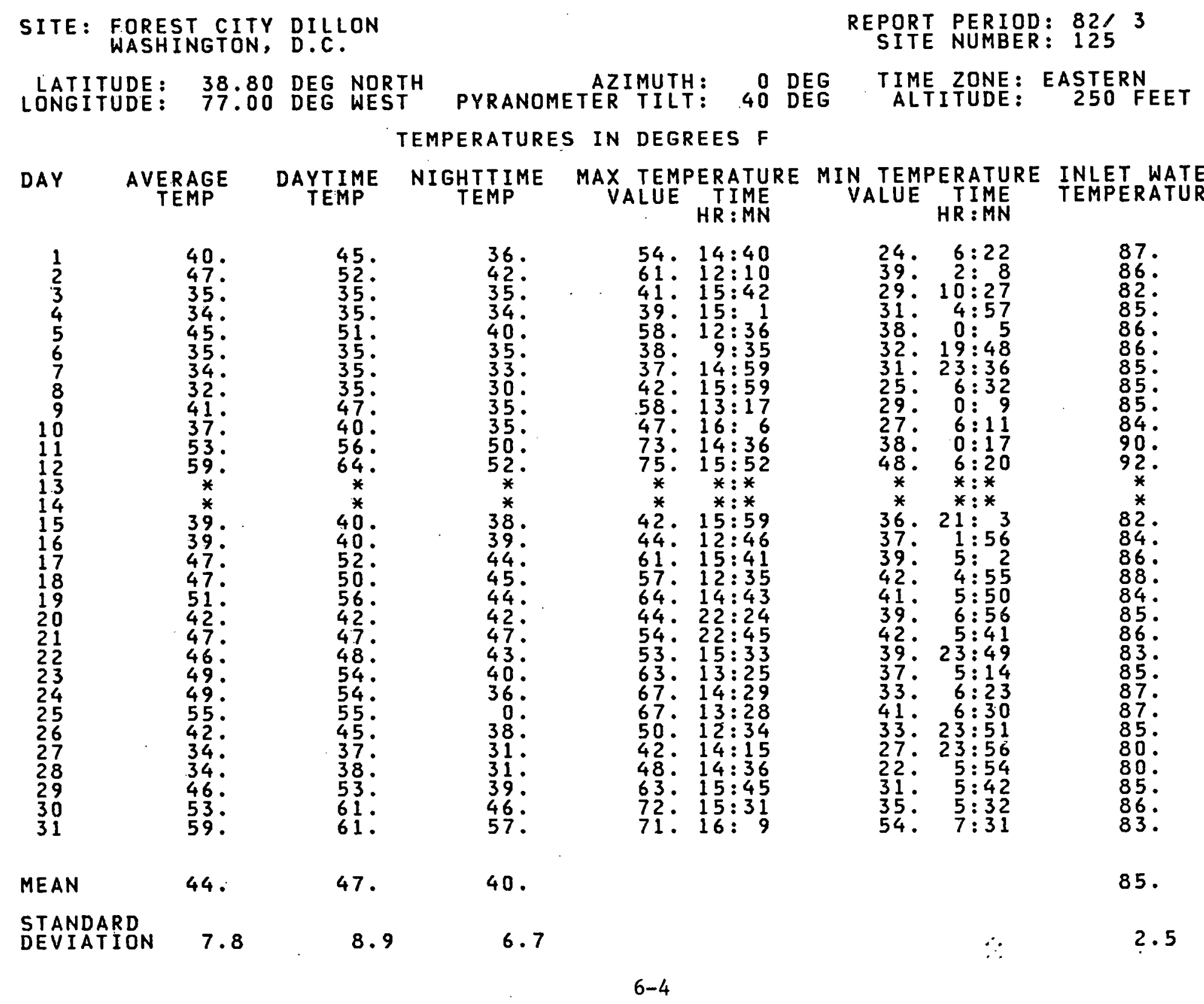


SITE : ROBERTS HOME
RESTON, VIRGINIA

LATITUDE: $\quad 38.90$ DEG NORTH
PYRANOMETER TILIT: $\quad 90$ DEG INSOLATION

DAY TOTAL TOTAL HOPIZONTAL TOTAL DIR INERTICAL HORIZONTAL TOTAL DIR $\begin{array}{ccc}\text { INSOLATION INSOLATION } & \text { INSOLATION } \\ \text { BTU\%FT2 } & \text { BTU/FT2 } & \text { BTU/FT2 }\end{array}$

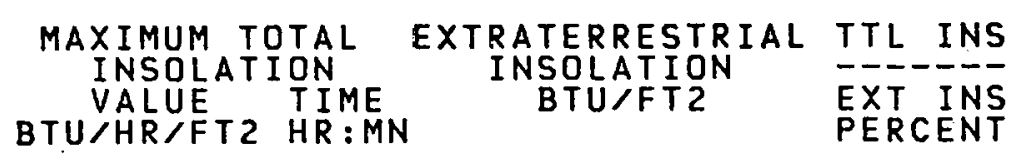

\begin{tabular}{|c|c|}
\hline 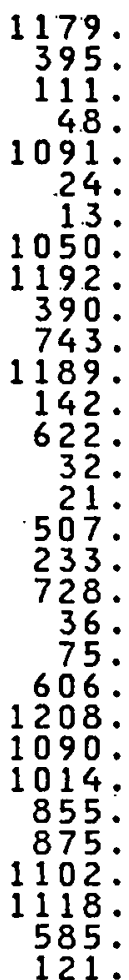 & 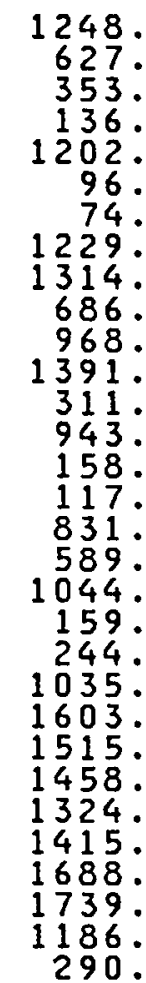 \\
\hline
\end{tabular}

N.A.

N.A.

N.A.

N.A.

N.A.

N.A.

$N \cdot A$.

N.A.

N.A.

N.A.

N.A.

N.A.

N.A.

N.A.

$N \cdot A$.

N.A.

N.A.

N.A.

N.A.

N.A.

N.A.

$N \cdot A$.

N.A.

N.A.

N.A.

N.A.

N.A.

N.A.

REPORT PERIOD: $82 / 3$
SITE NUMBER: 243

TIME ZONE:
ALTITUTERN
400 FEET

\begin{tabular}{|c|}
\hline 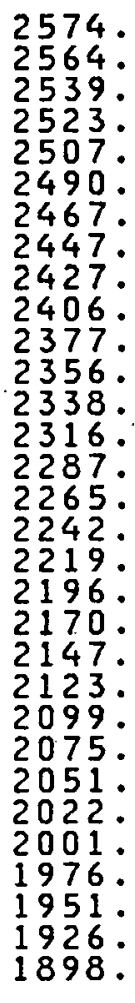 \\
\hline
\end{tabular}

46.

$156 \cdot 12: 17$

40 . $12: 26$

$27 \cdot \quad 12: 12$

$217 \cdot \quad 11: 53$

9. $14: 41$

$232^{\circ} \quad 12: 27$

$217 \cdot \quad 12: 28$

$128.14: 37$

$214.12: 35$

$116.15: 42$

$119 . \quad 13: 11$

18 . $10: 57$

$2 \frac{1}{5} \quad 12: 12$

$13: 24$

67. 11:9

198. $11: 27$

$10: 50$

$14: 58$

$\begin{array}{ll}198 . & 13: 58 \\ 208 . & 11: 39\end{array}$

$192 . \quad 11: 55$

$189 \cdot \quad 12: 35$

$217 . \quad 12: 42$

$201^{\circ} \quad 12: 10$

$201 . \quad 12: 10$

$\begin{array}{ll}1255^{\circ} & 14: 55 \\ 92 . & 15: 38\end{array}$

N.A.

2.257

MEAN

593.

870.

N.A.

208 . 
ENVIRONMENTAL SUMMARY REPORT

SITE : ROBERTS HOME
RESTON, VIRGINIA

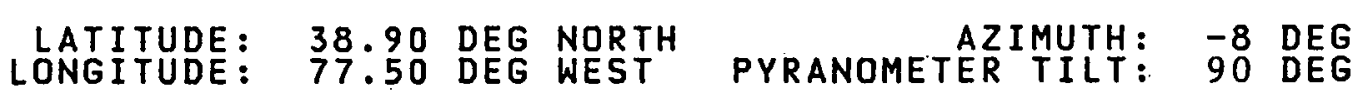

TEMPERATURES IN DEGREES F

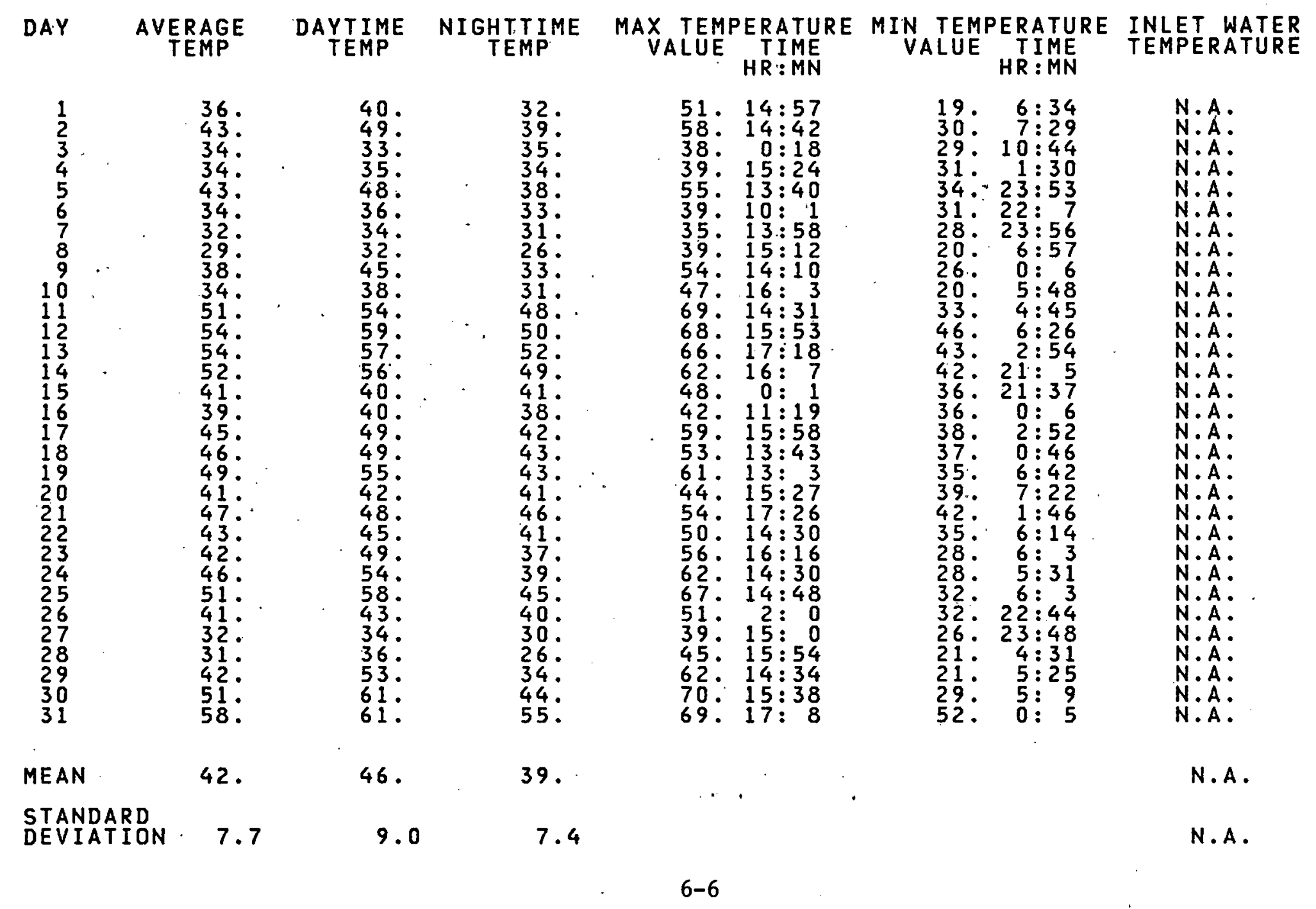
REPORT PERIOD: $82 / 3$
SITE NUMBER: 243 TIME ZONE:
ALTITUDE :
400 FEET ALTITUDE: 400 FEET

\section{ER}


SITE: ROBERTS HOME
RESTON, VIRGINIA

REPORT PERIOD: $82 / 3$

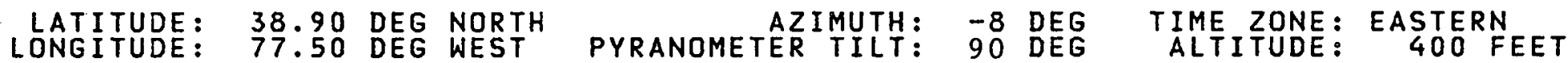
WIND: SPEED IN MPH-DIRECTION IN DEGREES

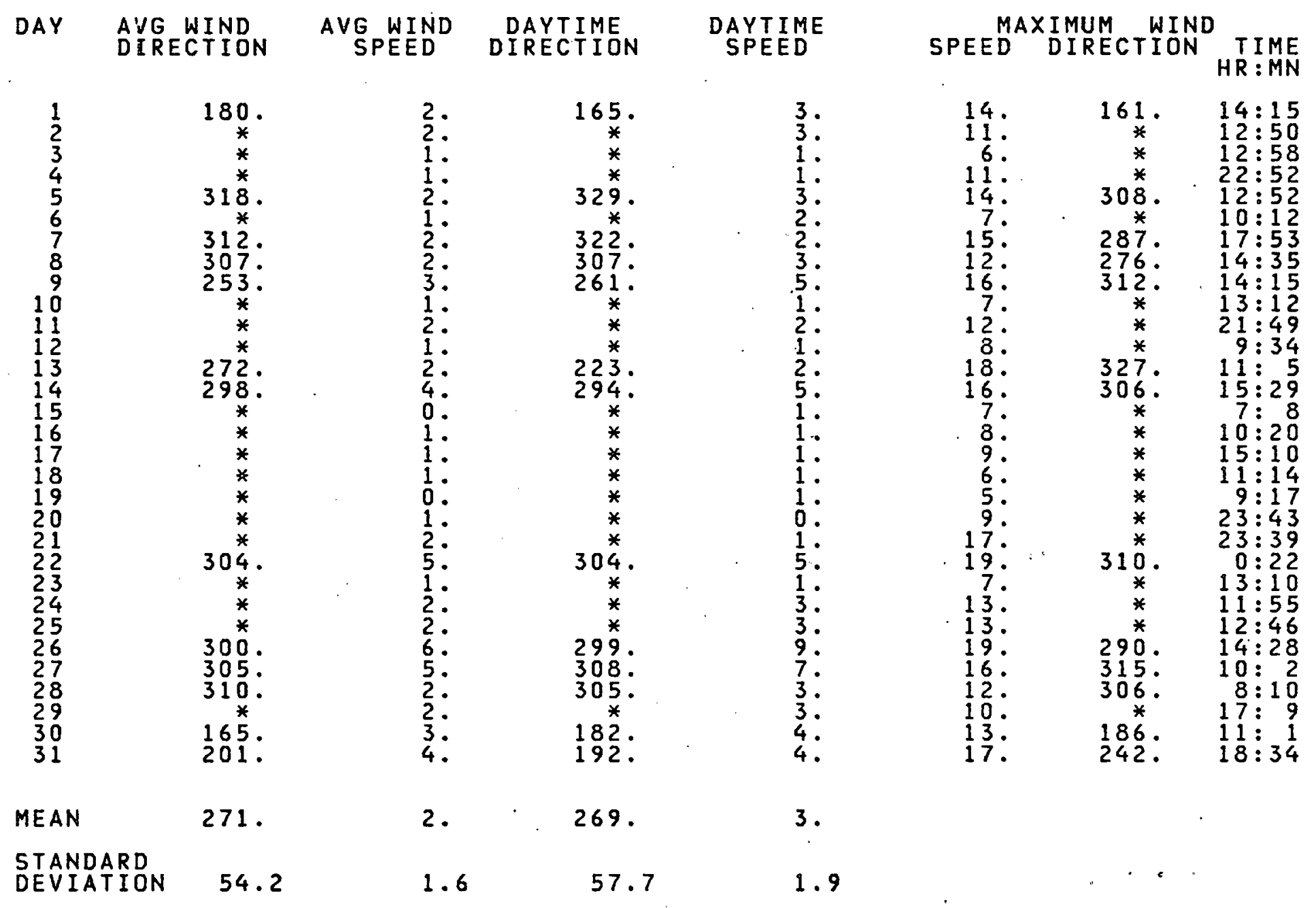


ENVIRONMENTAL SUMMARY REPORT

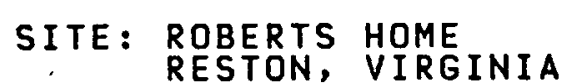

REPORT PERIOD: $82 / 3$

LATITUDE: 38.90 DEG NORTH AZIMUTH: -8 DEG TIME ZONE: EASTERN LONGITUDE: 77.50 DEG WEST PYRANOMETER TILT: 90 DEG ALTITUDE: 400 FEET WIND: NUMBER OF HOURS IN EACH VELOCITY INTERVAL

\begin{tabular}{|c|c|c|c|c|c|c|c|c|c|}
\hline DAY & $\begin{array}{l}0-3 \\
\text { MPH }\end{array}$ & $\begin{array}{l}4-7 \\
M P H\end{array}$ & $\begin{array}{l}8-12 \\
M P H\end{array}$ & $\begin{array}{c}13-18 \\
M P H\end{array}$ & $\begin{array}{l}19-24 \\
M P H\end{array}$ & $\begin{array}{l}25-31 \\
M P H\end{array}$ & $\begin{array}{c}32-38 \\
\mathrm{MPH}\end{array}$ & $\begin{array}{c}A B O V E \\
38\end{array}$ & $\begin{array}{c}\text { MISSING } \\
\text { HOURS }\end{array}$ \\
\hline $\begin{array}{r}1 \\
2 \\
3 \\
4 \\
5 \\
6 \\
7 \\
8 \\
9 \\
10 \\
11 \\
12 \\
13 \\
14 \\
15 \\
16 \\
17 \\
18 \\
199 \\
20 \\
21 \\
22 \\
23 \\
24 \\
25 \\
26 \\
27 \\
28 \\
29 \\
30 \\
31\end{array}$ & $\begin{array}{r}19 \\
19 \\
24 \\
24 \\
19 \\
24 \\
16 \\
20 \\
15 \\
23 \\
199 \\
23 \\
16 \\
12 \\
24 \\
24 \\
23 \\
24 \\
24 \\
24 \\
21 \\
8 \\
24 \\
19 \\
19 \\
2 \\
6 \\
23 \\
22 \\
14 \\
12\end{array}$ & $\begin{array}{r}5 \\
5 \\
0 \\
0 \\
5 \\
0 \\
8 \\
4 \\
8 \\
1 \\
5 \\
1 \\
8 \\
11 \\
10 \\
0 \\
1 \\
0 \\
0 \\
0 \\
2 \\
16 \\
0 \\
5 \\
5 \\
16 \\
15 \\
1 \\
2 \\
10 \\
11\end{array}$ & $\begin{array}{l}0 \\
0 \\
0 \\
0 \\
0 \\
0 \\
0 \\
0 \\
0 \\
0 \\
1\end{array}$ & $\begin{array}{l}0 \\
0 \\
0 \\
0 \\
0 \\
0 \\
0 \\
0 \\
0 \\
0 \\
0 \\
0 \\
0 \\
0 \\
0 \\
0 \\
0 \\
0 \\
0 \\
0 \\
0 \\
0 \\
0 \\
0 \\
0 \\
0 \\
0 \\
0 \\
0 \\
0 \\
0 \\
0\end{array}$ & $\begin{array}{l}0 \\
0 \\
0 \\
0 \\
0 \\
0 \\
0 \\
0 \\
0 \\
0 \\
0 \\
0 \\
0 \\
0 \\
0 \\
0 \\
0 \\
0 \\
0 \\
0 \\
0 \\
0 \\
0 \\
0 \\
0 \\
0\end{array}$ & $\begin{array}{l}0 \\
0 \\
0 \\
0 \\
0 \\
0 \\
0 \\
0 \\
0 \\
0 \\
0 \\
0 \\
0 \\
0 \\
0 \\
0 \\
0 \\
0 \\
0 \\
0 \\
0 \\
0 \\
0 \\
0 \\
0 \\
0 \\
0 \\
0\end{array}$ & $\begin{array}{l}0 \\
0 \\
0 \\
0 \\
0\end{array}$ & $\begin{array}{l}0^{\prime} \\
0 \\
0 \\
0 \\
0 \\
0 \\
0 \\
0 \\
0 \\
0 \\
0 \\
0 \\
0 \\
0 \\
0 \\
0 \\
0 \\
0 \\
0 \\
0 \\
0 \\
0\end{array}$ & $\begin{array}{l}0 \\
0 \\
0 \\
0 \\
0 \\
0 \\
0 \\
0 \\
0 \\
0 \\
0 \\
0 \\
0 \\
0 \\
0 \\
0 \\
0 \\
0 \\
0 \\
0\end{array}$ \\
\hline RAGE & 18.9 & 4.7 & 0.4 & 0.0 & 0.0 & 0.0 & 0.0 & 0.0 & 0.0 \\
\hline TAL & 586 & 145 & 12 & 0 & 0 & 0 & 0 & 0 & 1 \\
\hline
\end{tabular}


ENVIRONMENTAL SUMMARY REPORT

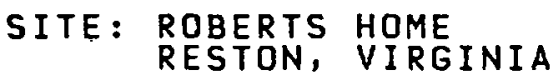

\begin{tabular}{lll} 
LATITUDE: & 38.90 & DEG NORTH \\
LONGITUDE: & 77.50 DEG WEST PYRANOMETER TILT: & -8 DEG \\
\hline
\end{tabular} RELATIVE HUMIDITY IN PER CENT

$\begin{array}{cr}\text { DAY } & \text { RELATIV } \\ & \text { HUMIDITY } \\ & \\ 1 & 45 . \\ 2 & 41 . \\ 3 & 49 . \\ 4 & 58 . \\ 5 & 58 . \\ 6 & 60 . \\ 7 & 88 . \\ 8 & 58 . \\ 9 & 44 . \\ 10 & 49 . \\ 11 & 66 . \\ 12 & 64 . \\ 13 & 64 . \\ 14 & 20 . \\ 15 & 58 . \\ 16 & 81 . \\ 17 & 76 . \\ 18 & 62 . \\ 19 & 60 . \\ 20 & 82 . \\ 21 & 85 . \\ 22 & 44 . \\ 23 & 46 . \\ 24 & 48 . \\ 25 & 58 . \\ 26 & 50 . \\ 27 & 36 . \\ 28 & 31 . \\ 29 & 30 . \\ 30 & 46 . \\ 31 & 73 . \\ & \\ \text { MEAN } & 56 . \\ \text { STANDARD } & \\ 0 E V 1 & \end{array}$

DEVIATION 17.

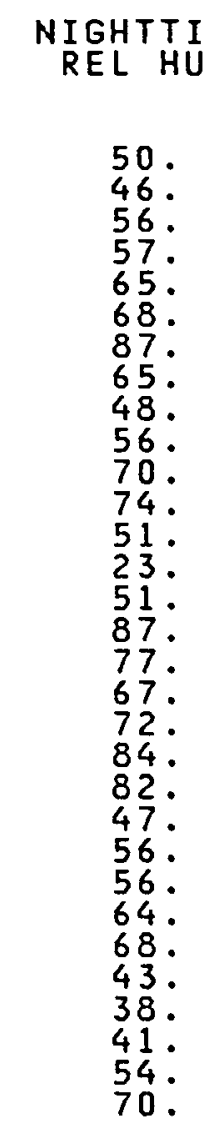
MAXIMUM HUMIDITY $H R: M N$

\section{MINIMUM HUMIDITY}

$\begin{array}{rr}73 \\ 63 \\ 75\end{array} \quad 23: 52$

$75 . \quad 3: 56$

$78.23: 3$

82. $\quad 6: 9$

84. $\quad 23: 5$

82. $0: 1$

$71 . \quad 7: 28$

$74 . \quad 23: 57$

$90 . \quad 7: 47$

$5: 22$

96. $11: 53$

$33.21: 5$

83. $22: 14$

99. $21: 21$

$97.5: 53$

$73 . \quad 1: 40$

88 . $\quad 5: 59$

$23: 48$

$20: 49$

$6: 51$

$6: 30$

$6: 19$

$7: 34$

$2: 48$

$1: 40$

$23: 28$

$6: 8$

$5: 52$

$18: 44$

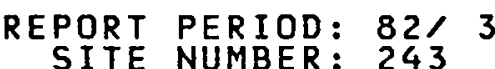

TIME ZDNE: EASTERN

ALTITUDE: 400 FEET 


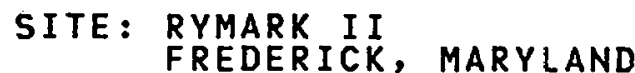
$\begin{array}{ll}\text { LATITUDE: } & 39.30 \\ \text { LONGITUDE: } & 77.25 \text { DEG NORTH }\end{array}$

DAY TOTAL VERTICAL TOTAL DIFF TOTAL DIR INSOLATION INSOLATION BNSOLATION

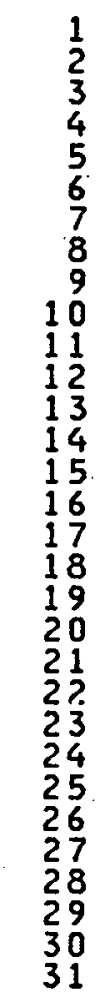

N.A.

N.A.

N.A.

N.A:

N.A.

N.A.

N.A.

$1688^{\circ}$.

$1254^{\circ}$.

N.A.

N.A.

1258 .

281 .

1221 .

168 .

580 .

431.

$153^{\circ}$

129.

145.1 .

$1070^{\circ}$.

854 .

1208 .

1476.

1467.

832.

N.A.

N.A.

N.A:

N:A.

N.A.

N.A.

N.A.

N.A.

N.A.

N.A.

N.A.

N.A.

N.A.

N.A.

N.A:

N.A.

N.A.

N.A.

N.A.

N.A.

N.A.

N.A.

N.A.

N.A.

N.A.

N.A.

N.A.

N.A.

N.A.

N.A.

N.A.

N.A.

N.A.

N.A.

N.A.

N.A.

N.A.

N.A.

N.A.

N.A.

N.A.

N.A.

N.A.

$\begin{array}{lll}\text { AZIMUTH : } & -9 & \text { DEG } \\ & 90 & \text { DEG }\end{array}$

REPORT PERIOD: $82 / 3$

SITE NUMBER: 224 S

TIME ZONE: EASTERN
ALTITUDE : 2.90 FEET

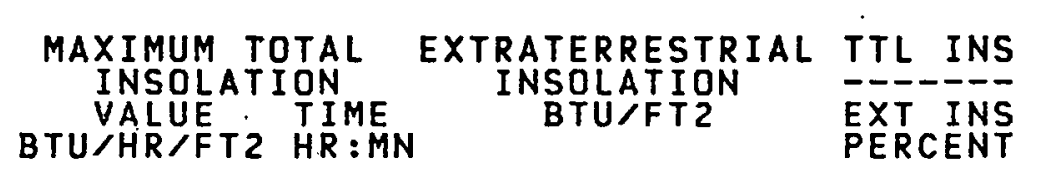

MEAN

783.

N.A.

N.A.

$12: 31$

2580.

2550 .

256. $12: 40$

17. $12: 49$

$248 . \quad 12: 8$

52. $\quad 12: 39$

256. $11: 27$

239. $11: 54$

$150^{\circ} \quad 14: 31$

225. $11: 35$

$239 . \quad 11: 53$

253. $\quad 14: 46$

46. $10: 45$

23. $\quad 11: 33$

178. $\quad 13: 53$

$138 . \quad 11: 33$

92: $11: 38$

55.
158.

158. $12: 26$

$242 . \quad 11 \vdots 4 \frac{1}{5}$

$207^{\circ} \quad 11: 29$

$239 . \quad 10: 54$

248. $10: 48$

227. $10: 52$

$219 . \quad 10: 47$

153: $13: 30$

95. $15: 23$

N.A.

N.A.

253.4 .

2492 .

2473 .

2453 .

2433.

2405.

2385.

2365

$2326^{\circ}$.

$2298^{\circ}$.

2276 .

2254 .

2232 .

2210.

$2159^{\circ}$

2136.

2113.

2090 .

2066 .

.

1993.

$1970^{\circ}$

1970.

1945.

52.

STANDARD

546.

2269.

203. 
SITE: $\begin{aligned} & \text { RYMARK II } \\ & \text { FREDERICK, MARYLAND }\end{aligned}$

LATITUDE : 39.30 DEG NORT
LONGITUDE :
77.25 DEG WEST
REPORT PERIOD: $82 / 3$

TIME ZONE: EASTERN

ALTITUDE: 290 FEET

TEMPERATURES IN DEGREES F

\begin{tabular}{|c|c|c|c|c|c|c|c|}
\hline DAY & $\begin{array}{c}\text { AVERAGE } \\
\text { TEMP }\end{array}$ & $\begin{array}{c}\text { DAYT IME } \\
\text { TEMP }\end{array}$ & $\underset{\text { TEMP }}{N I G H T T M E}$ & $\begin{array}{c}\text { MAX TEMPERATURE } \\
\text { VALUE TIME } \\
\text { HR:MN }\end{array}$ & $\begin{array}{c}\text { MIN TEMP } \\
\text { VALUE }\end{array}$ & $\begin{array}{l}\text { ERATURE } \\
\text { TIME } \\
\text { HR:MN }\end{array}$ & $\begin{array}{l}\text { INLET WATER } \\
\text { TEMPERATURE }\end{array}$ \\
\hline $\begin{array}{l}1 \\
2 \\
3 \\
4 \\
5 \\
6 \\
7 \\
8 \\
9 \\
10 \\
111 \\
12 \\
13 \\
14 \\
15 \\
16 \\
17 \\
18 \\
19 \\
20 \\
21 \\
22 \\
23 \\
24 \\
25 \\
26 \\
27 \\
28 \\
29 \\
30 \\
31\end{array}$ & 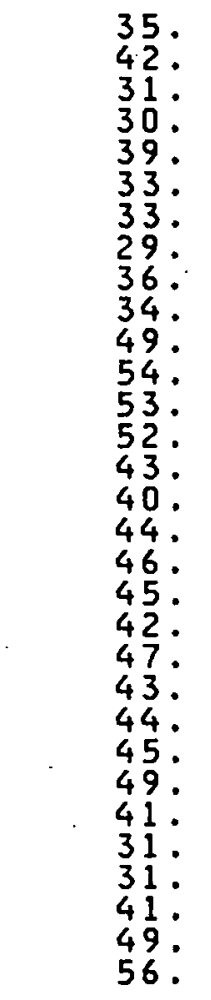 & 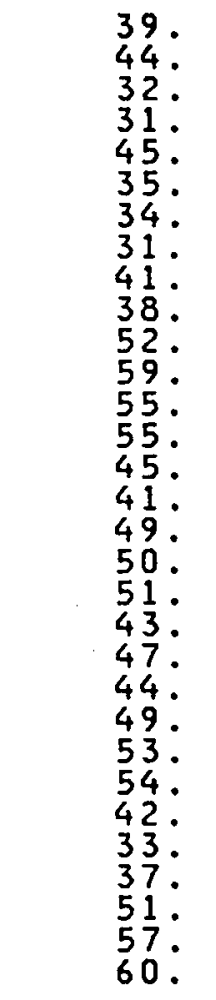 & 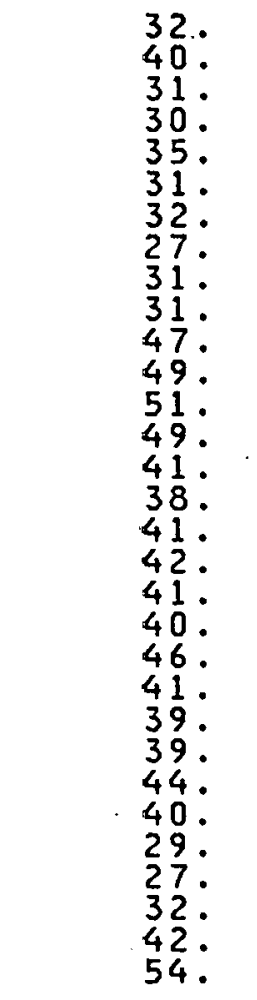 & 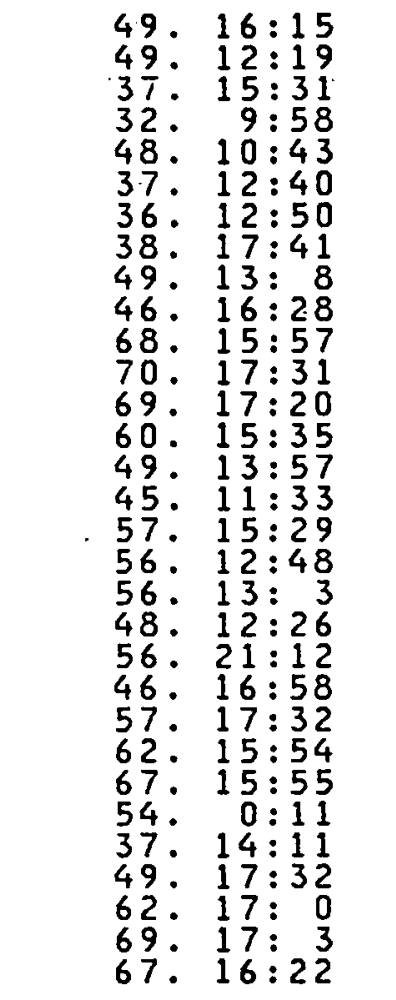 & 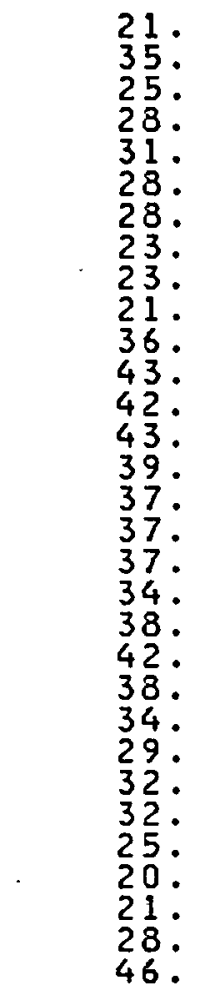 & $\begin{array}{r}6: 31 \\
7: 5 \\
21: 12 \\
2: 32 \\
23: 58 \\
2: 32 \\
23: 57 \\
6: 21 \\
0: 5 \\
6: 2 \\
0: 1 \\
23: 50 \\
3: 39 \\
22: 15 \\
22: 35 \\
19: 49 \\
0: 10 \\
0: 11 \\
6: 24 \\
0: 15 \\
0: 10 \\
6: 21 \\
23: 50 \\
5: 58 \\
5: 30 \\
22: 27 \\
23: 36 \\
5: 22 \\
5: 6 \\
6: 54 \\
1: 35\end{array}$ & 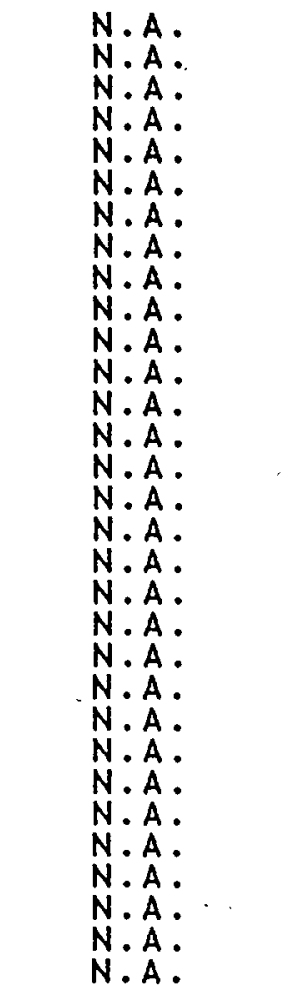 \\
\hline MEAN & 41. & 45. & 38. & & & & N.A. \\
\hline $1 \mathrm{~A}$ & 7.6 & 8.6 & 7.4 & & & & N.A. \\
\hline
\end{tabular}


ENVIRONMENTAL SUMMARY REPORT

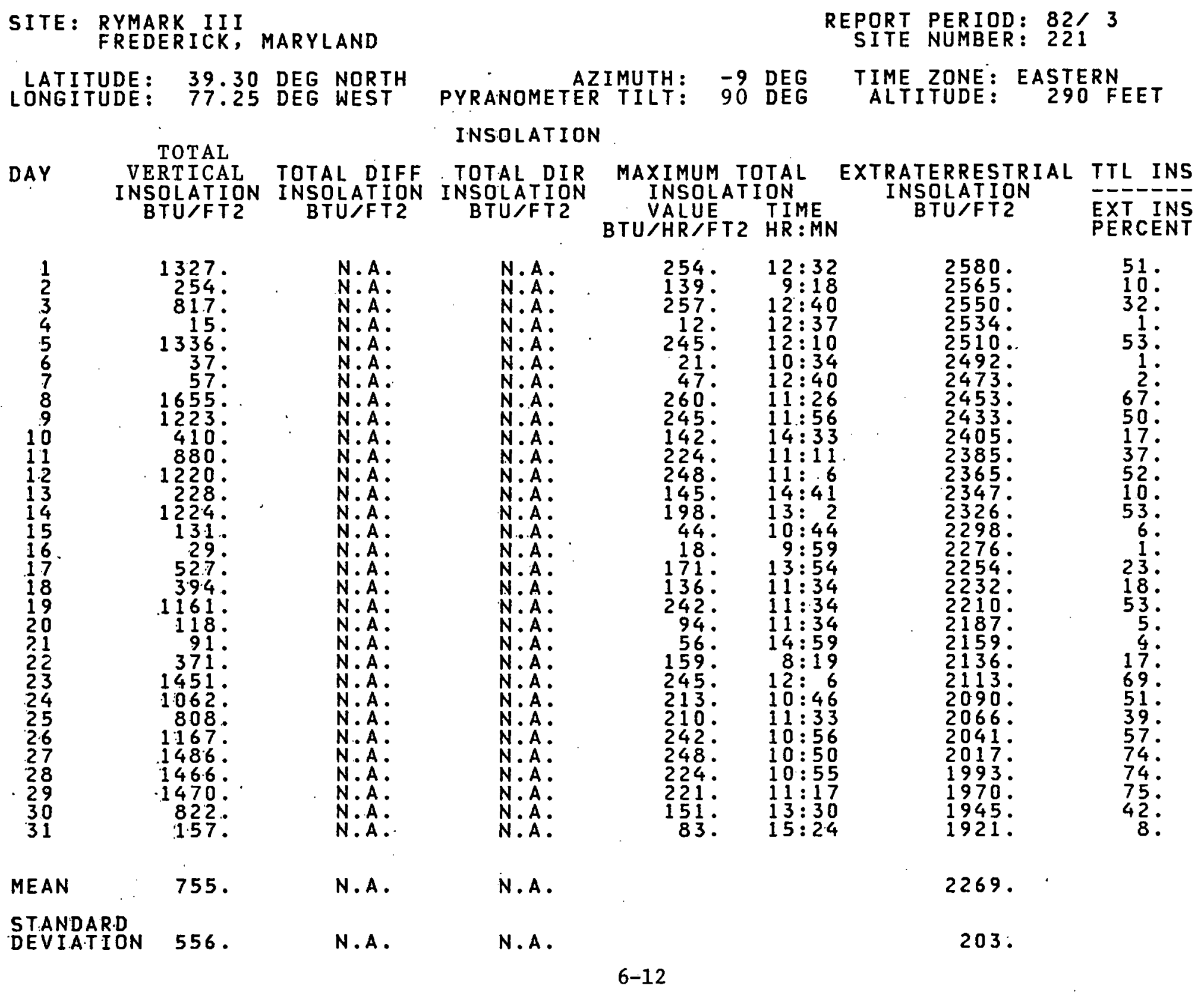


SITE: RYMARK III

REPORT PERIOD: $82 / 3$
SITE NUMBER: 321 $\begin{array}{llll}\text { LATITJDE: } & 39.30 & \text { DEG NORTH } \\ \text { LONGITIDE: } & 77.25 \text { DEG WEST PYRANOMETER TILT: } & -90 \text { DEG DEG TIME ZONE: EASTERN }\end{array}$ TEMPERATURES IN DEGREES F

\begin{tabular}{|c|c|c|c|c|c|c|}
\hline DAY & $\begin{array}{l}\text { AVERAGE } \\
\text { TEMP }\end{array}$ & $\begin{array}{l}\text { DAYTIME } \\
\text { TEYPP }\end{array}$ & $\underset{\text { TEMP }}{\text { NIGHTIME }}$ & $\begin{array}{c}\text { MAX TEMPERATURE } \\
\text { VALUE } T \text { TIME } \\
\text { HR:MN }\end{array}$ & $\begin{array}{c}\text { MIN TEMPERATURE } \\
\text { VALUE TIME } \\
\text { HR:MN }\end{array}$ & $\begin{array}{l}\text { INLET WATER } \\
\text { TEMPERATURE }\end{array}$ \\
\hline $\begin{array}{l}1 \\
2 \\
3 \\
4 \\
5 \\
6 \\
7 \\
8 \\
99 \\
10 \\
11 \\
12 \\
13 \\
14 \\
15 \\
16 \\
17 \\
18 \\
19 \\
20 \\
21 \\
22 \\
23 \\
24 \\
25 \\
26 \\
27 \\
28 \\
29 \\
30 \\
31\end{array}$ & 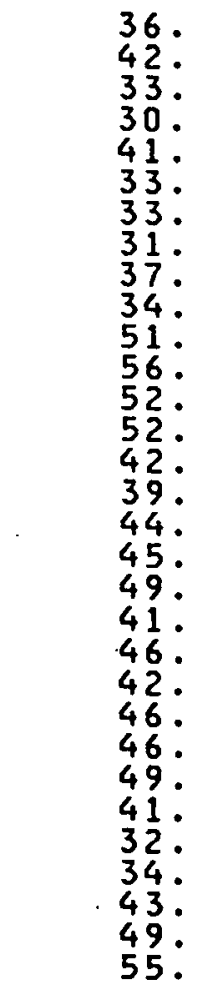 & 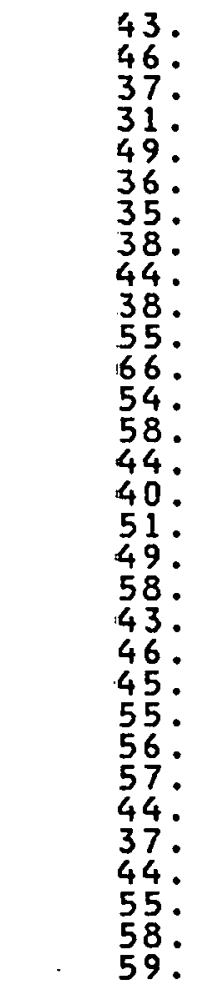 & 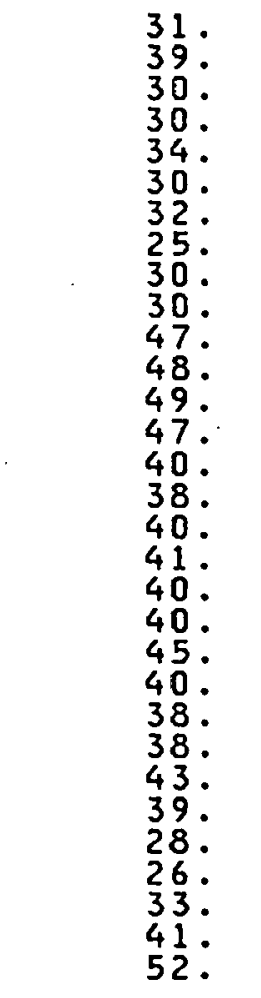 & 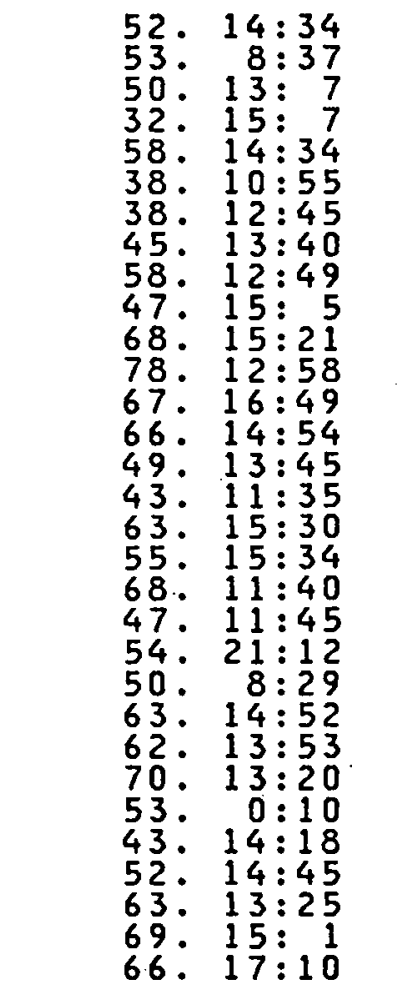 & 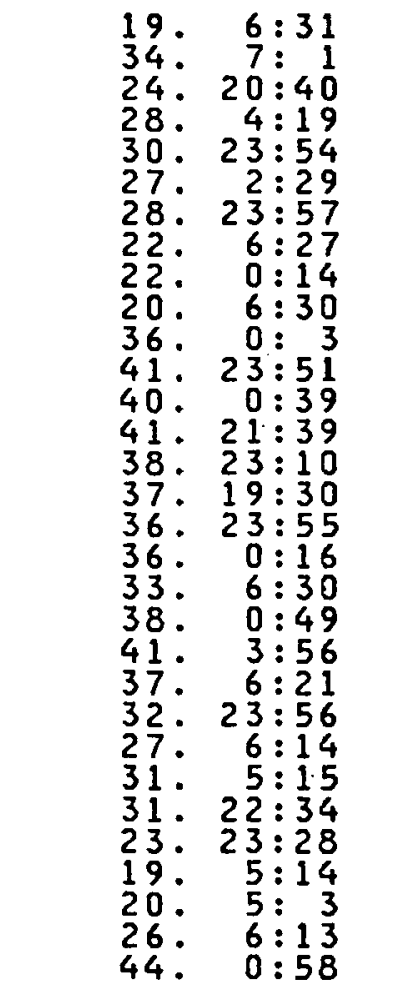 & 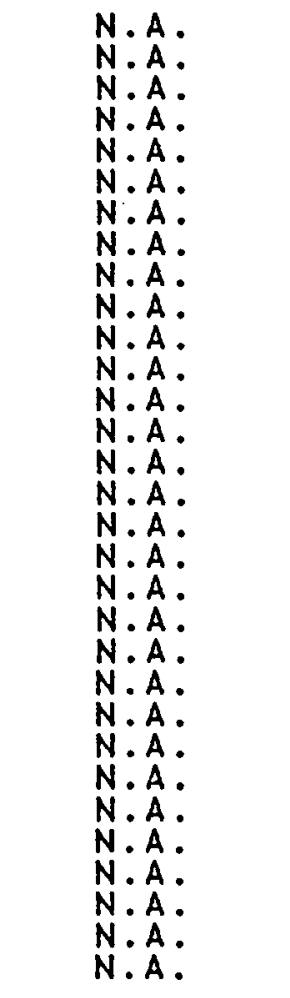 \\
\hline MEAN & 42. & 47. & 38. & & & N.A. \\
\hline Ev & 7.0 & 8.9 & 7.2 & & & N.A. \\
\hline
\end{tabular}


ENVIRONMENTAL SUMMARY REPORT

SITE: VITRO TEST SITE

REPORT PERIOD: $82 / 3$
SITE NUMBER: 152

LATITUDE: 39.00 DEG NORTH LONGITUDE: 77.15 DEG WEST

PYRANOMETER TILT: $\quad 0$ DEG

INSOLATION

DAY HORIZONTAL TOTAL45 DEG TOTAL DIR INSOLATION INSOLATION INSOLATION

BTU /FT2

BTU/FT2

BTU/FT2

MAXIMUM TOTAL BTU/HR/FT2 HR:MN

EXTRATERRESTRIAL TTL INS

N.A.

1079: N.A.

N.A.

N.A.

2106 .

$1421^{*}$.

$1528^{*}$.

1418.

1140.

$50 \stackrel{*}{5}$.

505
1400

375.

$1260^{\circ}$.

825 .

1408 .

425.
$321^{\circ}$

1106 .

1889.

1679.

$1539^{\circ}$.

175.1 .

2045.

1454.

2106.

$217^{*} 9^{\circ}$

2027 .

1417 .

48 .

$1735^{\circ}$

302 .

1222.

1710 .

$346^{\circ}$.

263 .

1199.

2463.

2094.

1793 .

$2140^{\circ}$

2548 .

$1588^{\circ}$.

588.
408.

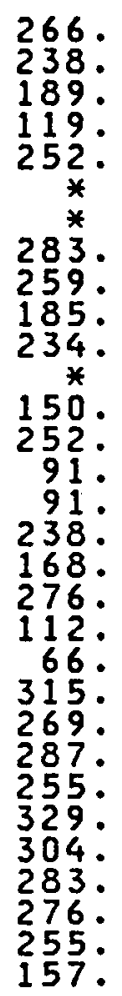

$12: 25$

INSOLATION

BTU/FT2

PET INS

30

449 .

1374.

N.A.

2136.

$14: 46$

$12: 40$

$11: 6$

*:*

N.A.

N.A.

N.A.

N.A.

N.A.

N.A.

N.A.

N.A.

N.A.

N.A.

N.A.

N.A.

N.A.

N.A.

N.A.

N.A.

N.A.

N.A.

N.A.

N.A.

$11: * 5$

$1 \frac{1}{12}: 45$

$14: 30$

$12: 14$

***

$15: 58$

$12: 57$

$11: 8$

$12: 20$

$13: 18$

$12: 53$

$11: 56$

$11: 29$

$14: 13$

$12: 17$

$1 \frac{1}{1}: 48$

$12: 53$

$12: 14$

$12: 4$

$11: 50$

(1)

13:34

$15: 45$

IME ZONE : EASTERN
ALTITUDE:

MEAN

1138.

777.

N.A .

$21790^{\circ} \quad 42$.

2224 .

2247.

$2270^{\circ}$.

2314.

2337.

$2381^{\circ}$

2404.

2427.

2448 .

2492 .

2515.

$2561^{\circ}$.

2584.

2605.

2648 .

2671.

2716 .

2736 .

2758 .

2780 .

STANDARD

DEVIATION 551 .

6-14

2470 .

202. 
ENVIRONMENTAL SUMMARY REPORT

SITE: VITRO TEST SITE

REPORT PERIOD: $82 / 3$

SITE NUMBER: 152

\section{LATITUDE: 39.00 DEG NORTH PYRANOMETAZIMUTH: 0 DEG TIME ZONE: EASTERN. \\ LONGITUDE: 77.15 DEG WEST PYRANOMETER TILT: O DEG ALTITUDE: 200 FEET}

TEMPERATURES IN DEGREES F

\begin{tabular}{|c|c|c|c|c|c|}
\hline DAY & $\begin{array}{l}\text { AVER.AGE } \\
\text { TEMP }\end{array}$ & $\begin{array}{c}\text { DAYTIME } \\
\text { TEMP }\end{array}$ & $\underset{\text { NEMP }}{\text { NIGHTTIME }}$ & $\begin{array}{c}\text { MAX TEMPERATURE } \\
\text { VALUE TIME } \\
\text { HR:MN }\end{array}$ & $\begin{array}{c}\text { MIN TEMPERATURE INLET WATER } \\
\text { VALUE TIME } \\
\text { HR:MN }\end{array}$ \\
\hline
\end{tabular}

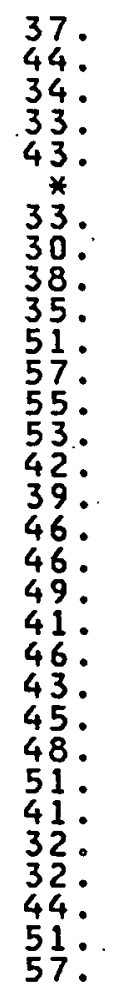

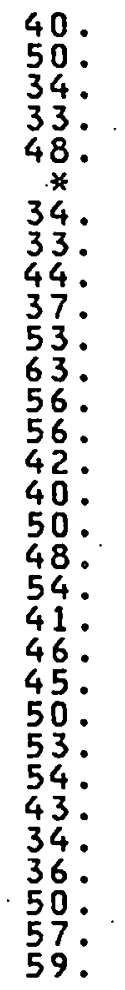

35.

38.

33 .

39.

32 .

28 .

33 .

$49^{\circ}$

52 .

$50^{\circ}$.

42 :

38 .

42.

$44^{\circ}$

41.

46.

39 .

43.

48.

40.

29.

28
37
46

49. $13: 45$

$58 \cdot 14: 33$
$42: 16: 59$

$42: 16: 59$

55. $11: 17$

$36.14:{ }^{*}: 23$

$39.15: 51$

$54: 14: 7$

46. 16:

$66.16: 35$

71. $15: 47$

$66 \cdot 16: 46$

48. $0: 4$

43. $12: 52$

63. $15: 15$

64: $13: 21$

44. $12: 55$

52. $22: 34$

59. 16:14

61. 14:45

64. 14:25

$39 \circ 14: 28$

$46^{\circ} 16: 28$

46. $16: 22$

$67 \cdot 16: 41$

MEAN

43.

46.

40.

22. $6: 46$

$32.7: 25$
$29 . \quad 9: 36$

$30.6: 24$

35 . $22: 29$

30. $23: * 8$

$23 \cdot 6: 44$

$28: \quad 0: 12$

24. 6:39

37. $0: 11$

$46.6: 45$

43 . $6: 2$

$35.22: 36$

37. $0: 1$

38: 4:41

41. $0: 8$

$37 . \quad 6: 18$

38. $\quad 7: 8$

$41 . \quad 5: 33$

$6: \frac{1}{2}$

33 . $5: 56$

$38 . \quad 4: 37$

$31 \cdot 22: 44$

25. $23: 58$

$\begin{array}{ll}20 & 5: 34 \\ 27 & 5: 10\end{array}$

$34.4: \frac{1}{4}$

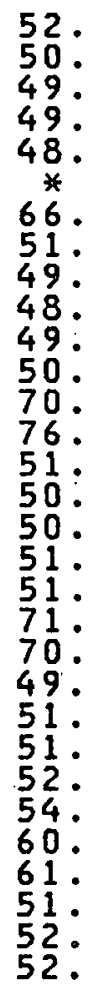

STANDARD

7.8

8.6

7.6

54.

8.0 
ENVIRONMENTAL SUMMARY REPORT

SITE: VITRO TEST SITE

REPORT PERIOD: $82 \prime 3$

LATITUDE: 39.00 DEG NORTH LONGITUDE: 77.15 DEG WEST

PYRANOMETER TILT: $\quad$ O DEG

TIME ZONE : EASTERN

ALTITUDE: 200 FEET

WIND: SPEED IN MPH-DIRECTION IN DEGREES

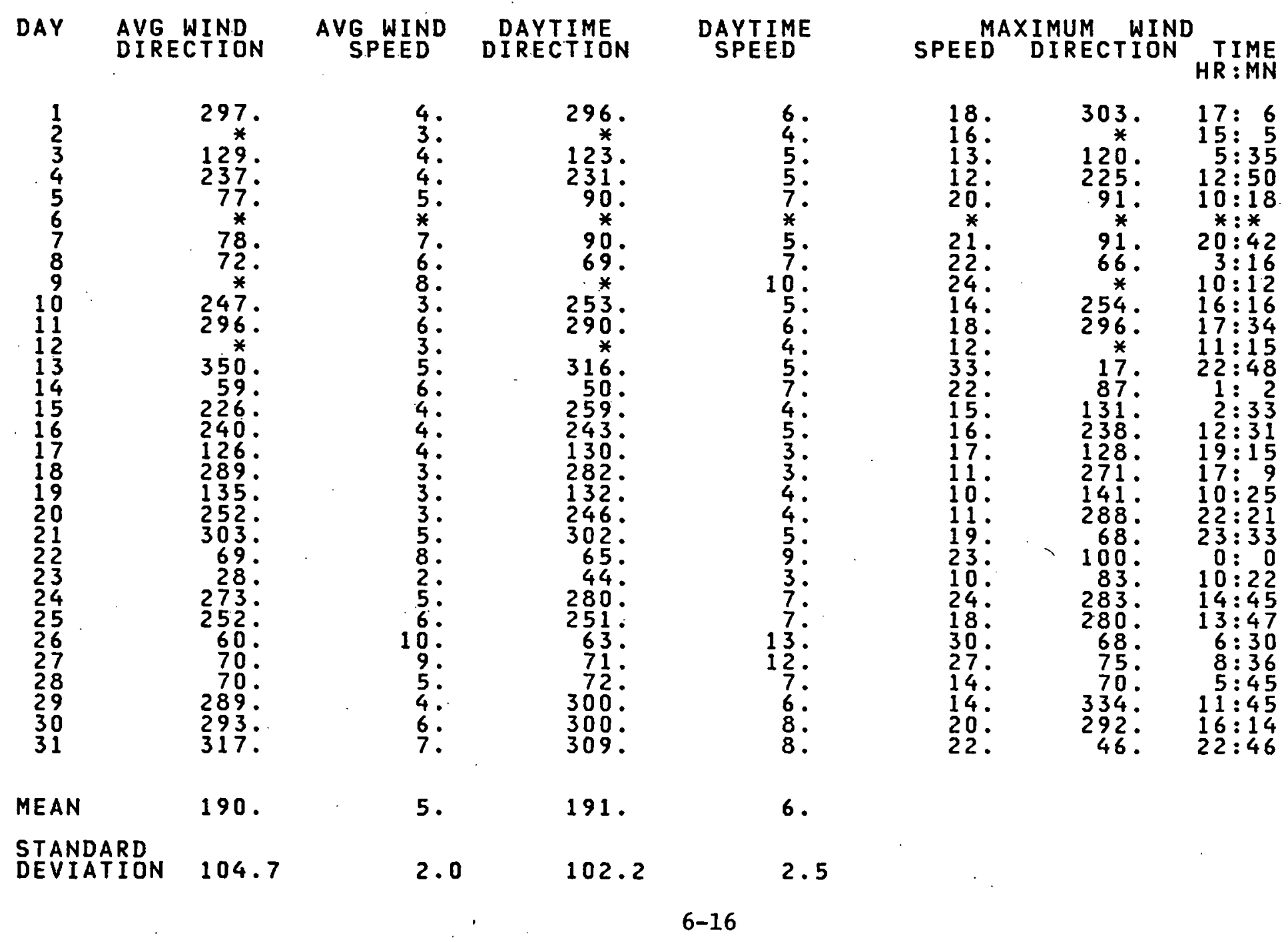


ENVIRONMENTAL SUMMARY REPORT

SITE: VITRO TEST SITE

REPORT PERIOD: $82 / 3$
SITE NUMBER: 152

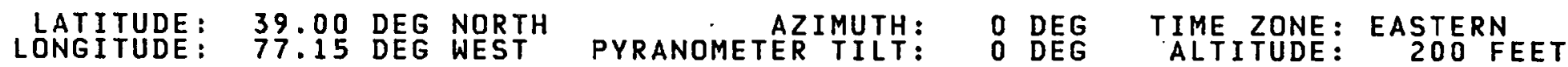

WIND: NUMBER OF HOURS IN EACH VELOCITY INTERVAL

\begin{tabular}{|c|c|c|c|c|c|c|c|c|c|}
\hline DAY & $\begin{array}{l}0-3 \\
M P H\end{array}$ & $\begin{array}{l}4-7 \\
M P H\end{array}$ & $8-12$ & $\begin{array}{l}13-18 \\
M P H\end{array}$ & $\begin{array}{c}19-24 \\
M P H\end{array}$ & $\underset{M P H}{25-31}$ & $\begin{array}{c}32-38 \\
\mathrm{MPH}\end{array}$ & $\underset{38}{A B Q V E}$ & $\begin{array}{c}\text { MISSING } \\
\text { HOURS }\end{array}$ \\
\hline $\begin{array}{l}1 \\
2 \\
3 \\
4 \\
5 \\
6 \\
7 \\
8 \\
9 \\
10 \\
11 \\
12 \\
13 \\
14 \\
15 \\
16 \\
17 \\
18 \\
19 \\
20 \\
21 \\
22 \\
23 \\
24 \\
25 \\
26 \\
27 \\
28 \\
29 \\
30 \\
31\end{array}$ & $\begin{array}{r}13 \\
14 \\
13 \\
11 \\
10 \\
0 \\
2 \\
7 \\
2 \\
214 \\
10 \\
16 \\
9 \\
8 \\
14 \\
15 \\
15 \\
15 \\
15 \\
12 \\
7 \\
2 \\
19 \\
11 \\
8 \\
0 \\
1 \\
18 \\
10 \\
7 \\
0\end{array}$ & $\begin{array}{r}5 \\
9 \\
11 \\
12 \\
7 \\
0 \\
6 \\
11 \\
7 \\
10 \\
7 \\
6 \\
11 \\
10 \\
99 \\
8 \\
7 \\
9 \\
99 \\
12 \\
14 \\
11 \\
5 \\
77 \\
10 \\
8 \\
8 \\
13 \\
11 \\
17 \\
16\end{array}$ & $\begin{array}{r}5 \\
1 \\
0 \\
1 \\
5 \\
0 \\
7 \\
6 \\
14 \\
0 \\
7 \\
0 \\
4 \\
6 \\
1 \\
1 \\
2 \\
0 \\
0 \\
0 \\
2 \\
11 \\
0 \\
6 \\
5 \\
9 \\
12 \\
3 \\
3 \\
10 \\
7\end{array}$ & $\begin{array}{l}0 \\
0 \\
0 \\
0 \\
0 \\
0 \\
0 \\
0 \\
0 \\
0 \\
1 \\
0 \\
0 \\
0 \\
0 \\
0 \\
0 \\
0 \\
0 \\
0 \\
0 \\
0 \\
0 \\
0 \\
0 \\
0 \\
1 \\
0 \\
0 \\
0 \\
0\end{array}$ & 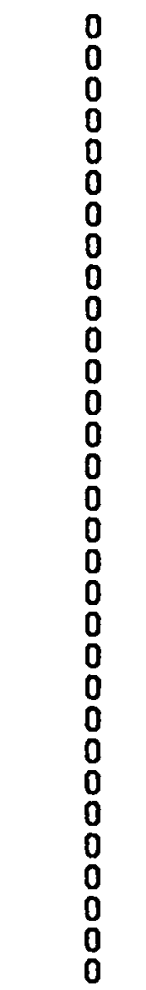 & 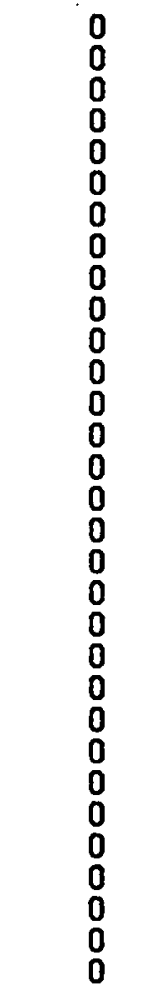 & $\begin{array}{l}0 \\
0 \\
0 \\
0 \\
0 \\
0 \\
0 \\
0 \\
0 \\
0 \\
0 \\
0 \\
0 \\
0 \\
0 \\
0 \\
0 \\
0 \\
0 \\
0 \\
0 \\
0 \\
0 \\
0 \\
0 \\
0 \\
0 \\
0 \\
0 \\
0 \\
0 \\
0 \\
\end{array}$ & 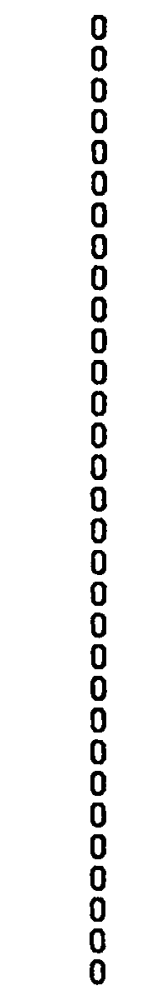 & $\begin{array}{r}1 \\
0 \\
0 \\
0 \\
2 \\
24 \\
9 \\
0 \\
0 \\
0 \\
0 \\
2 \\
0 \\
0 \\
0 \\
0 \\
0 \\
0 \\
0 \\
0 \\
0 \\
0 \\
0 \\
0 \\
0 \\
0 \\
0 \\
0 \\
0 \\
0 \\
1\end{array}$ \\
\hline AVERAGE & 9.3 & 8.9 & 4.1 & 0.4 & 0.0 & 0.0 & 0.0 & 0.0 & 1.3 \\
\hline TOTAL & 288 & 276 & 128 & 13 & 0 & 0 & 0 & 0 & 39 \\
\hline
\end{tabular}


2ONE 7

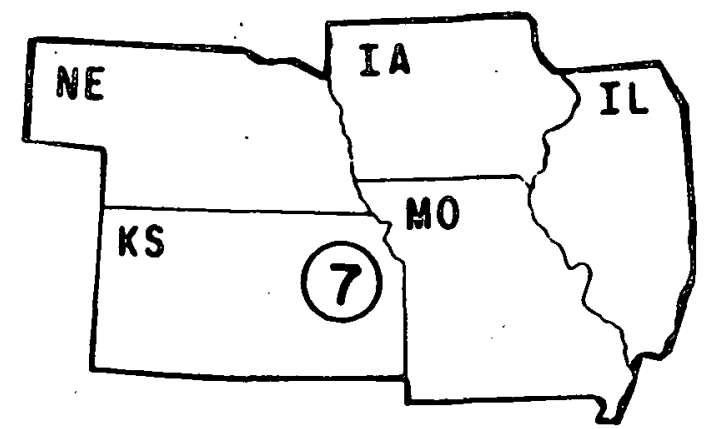


ENVIRONMENTAL SUMMARY REPORT

SITE : BOND CONSTRUCTION
REPORT PERIOD: $82 / 3$
SITE NUMBER: 132

\section{TIME ZONE: CENTRAL ALTITUDE: 800 FEET \\ LONGITUDE : 39.30 DEG NORTH \\ PYRANOMETER TILT: $\quad 37$ DEG INSOLATION}

DAY

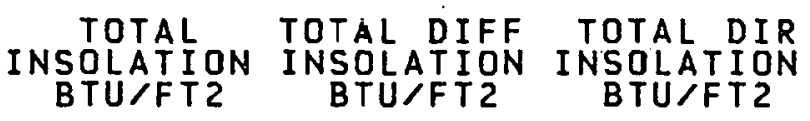

\section{MAXIMUM TOTAL INSOLATION VALUE TIME}

$\begin{array}{ll}1 \\ 2 \\ 3 \\ 4 \\ 5 \\ 6 \\ 7 \\ 8 \\ 9 \\ 10 \\ 11 \\ 112 \\ 13 \\ 14 \\ 15 \\ 16 \\ 17 \\ 18 \\ 19 \\ 20 \\ 21 \\ 22 \\ 23 \\ 24 \\ 25 \\ 25 \\ 2 & 7 \\ 2 & 8 \\ 2 & 9 \\ 3 & 0 \\ 3 & 1\end{array}$

1829 .

349.

621.

545.

2140 .

1506

1824.

1605 .

1418

1416.

1964 .

$1399^{\circ}$

1961.

1673.

1673.

809 .

2163

1690 .

2216

1375 .

$1692^{\circ}$.

2136

2207 .

067.

2286.

2266 .

N.A.

$N \cdot A$.

N.A.

N.A.

N.A.

N.A.

N.A.

N.A.

$N \cdot A$.

N.A.

$N \cdot A$.

N.A.

N.A.

N.A.

N.A.

$N \cdot A$.

N.A.

N.A.

N.A.

N.A.

N.A.

N.A.

N.A.

$N \cdot A$.

$N \cdot A$.

N.A.

N.A.

N.A.

N.A.

N.A.

N.A.

$N \cdot A$.

$N \cdot A$.

N.A.

N.A.

N.A.

N.A.

N.A.

N.A.

N.A.

N.A.

N.A.

N.A.

N.A.

N.A.

N.A.

$N \cdot A$.

N.A.

N.A.

N.A.

$N \cdot A$.

N.A.

N.A.

$290 . \quad 13: 56$

$117 . \quad 11: 52$

$23 . \quad 12: 45$

$244 \cdot 14: 23$

$320.13: 12$

$324.13: 30$

304. $14: 49$

$210 \cdot \quad 16: 13$

$317 \cdot \quad 13: 30$

$317 . \quad 13: 54$

$317 \quad 13: 3$

$33 . \quad 11: 52$

$317 \circ \quad 12: 46$

314.

314.

$12: 46$

$12: 5 \frac{1}{3}$

$13: 55$

$247 . \quad 14: 20$

$364.14: 21$

324. $12: 57$

324.

$12: 57$

$14: 47$

$13: 12$

$13: 19$

337.

317.

320 .

310 .

330.

$12: 42$

$12: 25$

$13: 56$

N.A.

N.A :

N.A.

N.A.

\section{XTRATERRESTRIAL TTL INS INSOLATION

BTU/FT2 EXT INS

\begin{tabular}{|c|c|}
\hline 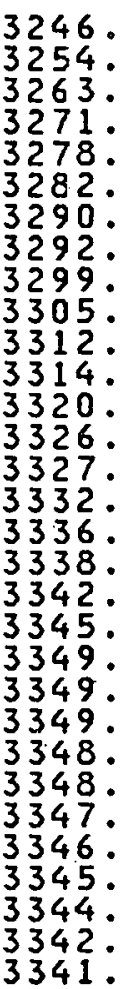 & $\begin{array}{l}56 . \\
11 . \\
2 . \\
19 \\
17 \\
65 . \\
46 . \\
55 . \\
21 . \\
49 . \\
43 . \\
13 . \\
59 . \\
2 . \\
42 . \\
59 . \\
50 . \\
11 . \\
24 . \\
65 . \\
50 . \\
66 . \\
41 . \\
42 . \\
51 . \\
64 . \\
66 . \\
62 . \\
46 . \\
68 . \\
68 .\end{array}$ \\
\hline
\end{tabular}

3319.

32 . 
ENVIRONMENTAL SUMMARY REPORT

SITE: BOND CONSTRUCTION
GLADSTONE," MISSOUR

REPORT PERIOD: $82 / 3$

SITE NUMBER: 132

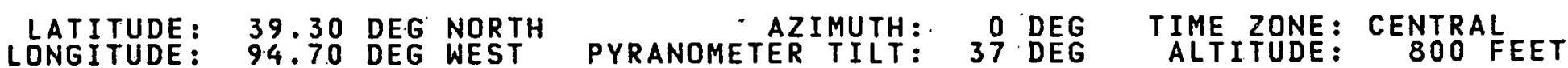
TEMPERATURES IN DEGREES F

\begin{tabular}{|c|c|c|c|c|c|}
\hline DAY & $\begin{array}{c}\text { AVERAGE } \\
\text { TEMP }\end{array}$ & $\begin{array}{c}\text { DAYTIME } \\
\text { TEMP }\end{array}$ & NIGHTTTIME & $\begin{array}{l}\text { MAX TEMPERATURE } \\
\text { VALUE TIME } \\
\text { HR:MN }\end{array}$ & $\begin{array}{c}\text { MIN TEMPERATURE INLET WATER } \\
\text { VALUE TTME } \\
\text { HR:MN }\end{array}$ \\
\hline
\end{tabular}

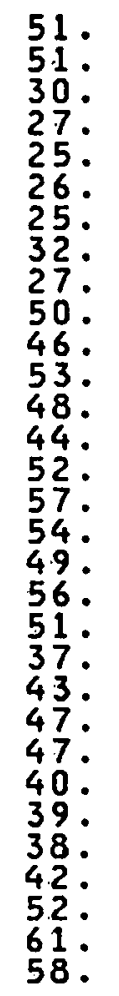

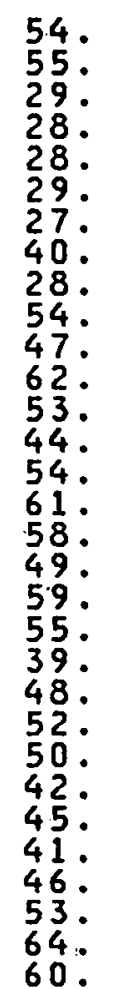

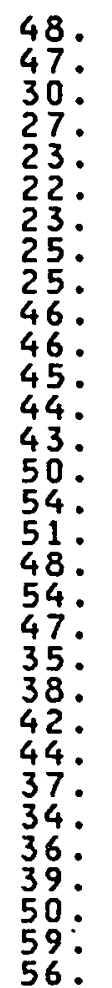

69. $15: 43$

$29.7: 29$

61.

$3 \frac{1}{34}: 15: 36$

$34: 14: 33$

$33.13: 12$

$42 \cdot 15: 36$

$37 \cdot 14: 29$

$50.14: 11$

$42.16: 13$

76. $16: 58$

$61: 16: 34$

65. $15: 27$

$48.15: 27$

69. $16: 46$

74. $16: 40$

78. $17: 9$

55. $16: 13$

$82 \cdot 16: 33$

$51: 16: 20$

60 . $16: 51$

62. 13:41

60. 15:30

50. 14:59

59. 16:10

50. $16: 10$

57: $16: 9$

65. $17: 45$

$79: 16: 36$

$27: \quad 8: 18$

22 : $8: 31$

$16 \cdot 23: 52$

11. $7 \vdots 315$

$20: 23: 58$

$16.4: 57$

24. $6: 27$

44. $\quad 7: 27$

40. $\quad 0: 2$

41: $23: 20$

$41.11: 1$

$44 \cdot 23: 52$

43. 7: 1

46. $5: 12$

$40: 23: 56$

$27.23: 56$

$31 \cdot 7: 4$

$31 . \quad 0: 46$

29:23:58

21. $6: 54$

30. $7: 17$

28. $5: 51$

39. $5: 7$

$\begin{array}{ll}47 & 4\end{array} \quad 6: 41$

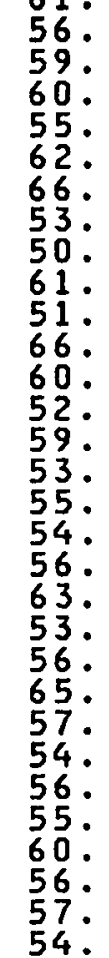

MEAN

47.

41.

57.

STANDARD

DEVIATION

44.

11.3

10.5

4.4 
SITE: LO-CAL

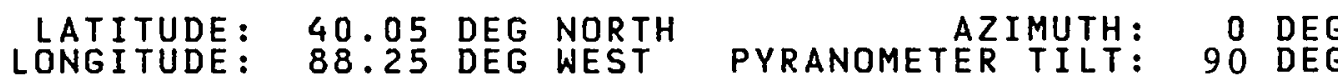

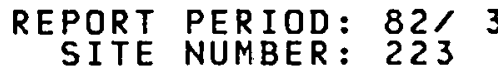

TIME ZONE: CENTRAL

ALTITUDE : 750 FEET

TOTAL HORTZONTAL INSOLATION

DAY VERTICAL TOTAL DIFF VERTICAL TOTAL DIFF TOTAL DIR
INSOLATION INSOLATION INSOLATION BTU/FT2 BTU/FT2 BTU/FT2
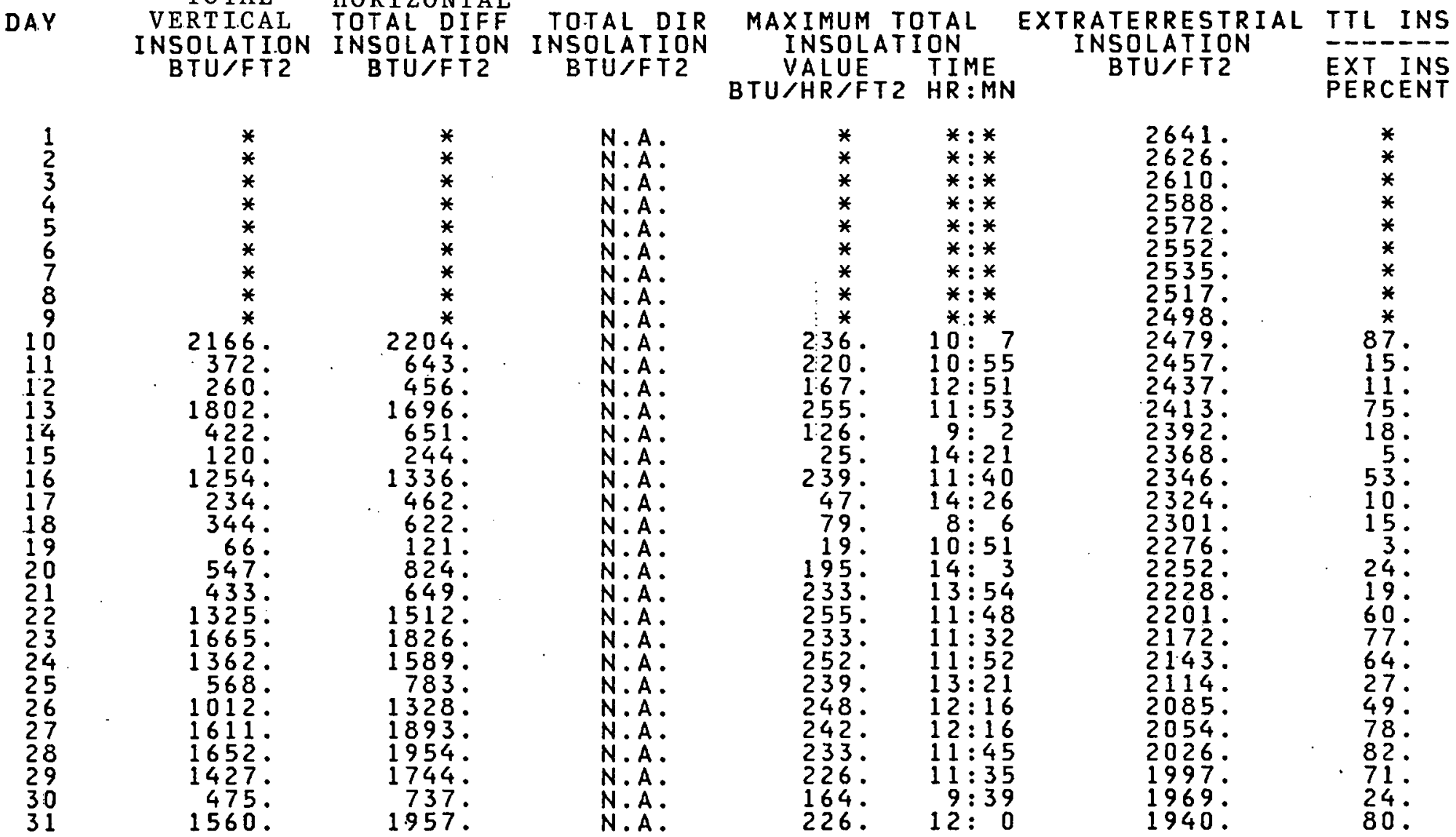

$2166^{*}$

372 .

1802 .

422.

1254 .

234 .

344 .

547 .

433 .

$1325^{\circ}$

$1665^{\circ}$.

1362 .

1012 .

1611 .

1652 .

1427.

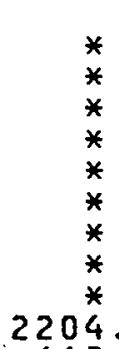

2204
643

456

1696 .

651.

1336

462 .

622.

824.

649

1512 .

1826.

1328 .

1893 .

1954

1744 .

1.957 .

$1147 . *$

$940 . *$

645 .

N.A.

N.A.

N.A.

N.A.

N.A.

N:A:

N.A:

N.A.

N.A.

N.A.

N.A.

N.A.

$N \cdot A$.

N.A.

N.A.

N.A.

N.A.

N.A.

$N \cdot A$.

N.A.

N.A.

N.A.

N.A.

N.A.

N.A.

N.A.

N.A. BTU/HR/FT2 HR:MN

TRATERRESTRIA
INSOLATION
BTUJFT2

N.A.

N.A. $\stackrel{*}{*} \quad * * *$

$\stackrel{*}{*}: *{ }^{*}$

$*: *$

$*: *$

$*: *$

$\stackrel{*}{*}: * *$

$* * *$

$10: 7$

$10: 55$

$12: 51$

$1 \frac{1}{9}: 53$

$9: 22$

$14: 21$

$11: 40$

$14: 26$

$10: 51$

$14: 3$

$13: 54$

$11: 48$

$11: 32$

$11: 52$

$13: 21$

$12: 16$

$12: 16$

$11: 45$

$11: 35$

$\begin{array}{ll}1644^{\circ} & 99: 39 \\ 226 . & 12: 0\end{array}$

2641.

2626.

2588 .

2572 .

2552.

2517 .

2498 .

2479 .

2457 .

2437.

2413.

2368 .

2346 .

2324.

2301.

2276.

2252.

2201 .

2172 .

2143 .

2114.

2054.

2026 .

1997 .

1969.

2326.

214.
STANDARD

DEVIATION 651 . 
ENVIRONMENTAL SUMMARY REPORT

SITE: LO-CAL

REPORT PERIOD: $82 / 3$

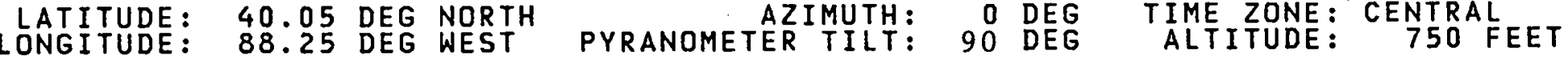

TEMPERATURES IN DEGREES F

\begin{tabular}{|c|c|c|c|c|c|}
\hline DAY & $\begin{array}{c}\text { AVERAGE } \\
\text { TEMP }\end{array}$ & $\begin{array}{c}\text { DAYTIME } \\
\text { TEMP }\end{array}$ & $\begin{array}{c}\text { NIGHTTIME } \\
\text { TEMP }\end{array}$ & $\begin{array}{c}\text { MAX TEMPERATURE } \\
\text { VALUE TIME } \\
\text { HR:MN }\end{array}$ & $\begin{array}{cc}\text { MIN TEMPERATURE } & \text { INLET WATER } \\
\text { VALUE TIME } & \text { TEMPERATURE } \\
\text { HR:MN } & \end{array}$ \\
\hline
\end{tabular}
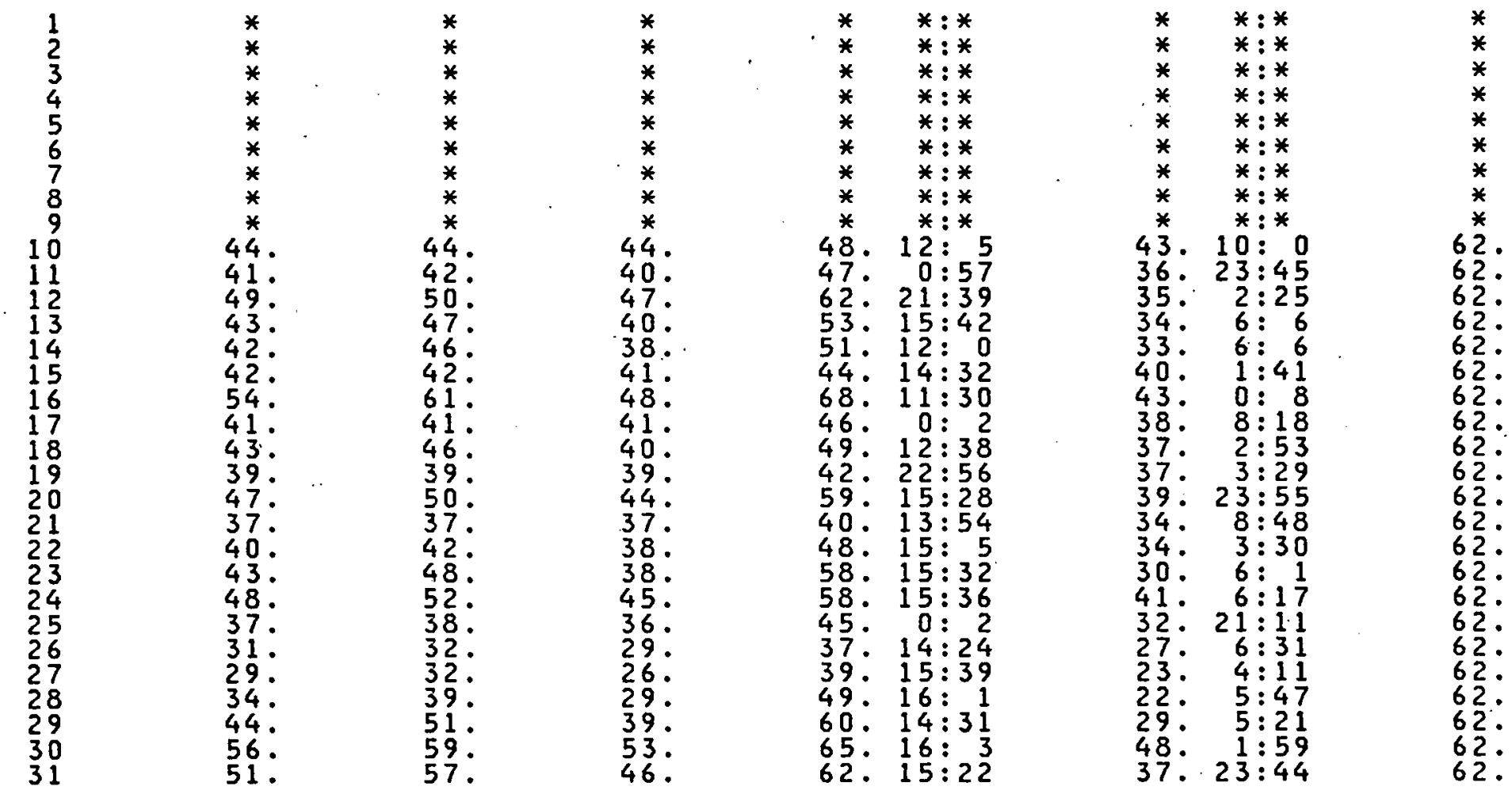

MEAN

42.*

$45 .^{*}$

$40 .^{*}$

$62 .^{*}$

STANDARD

$6.8 \quad 7.8 \quad 6.4$

0.1 
SITE: LO-CAL

REPORT PERIOD: $82 / 3$
SITE NUMBER: 223

LATITUDE: 40.05 DEG NORTH AZIMUTH: 0 DEG TIME ZONE: CENTRAL LONGITUDE: 88.25 DEG WEST PYRANOMETER TILT: 90 DEG ALTITUDE: 750 FEET WIND: SPEED IN MPH-DIRECTION IN DEGREES

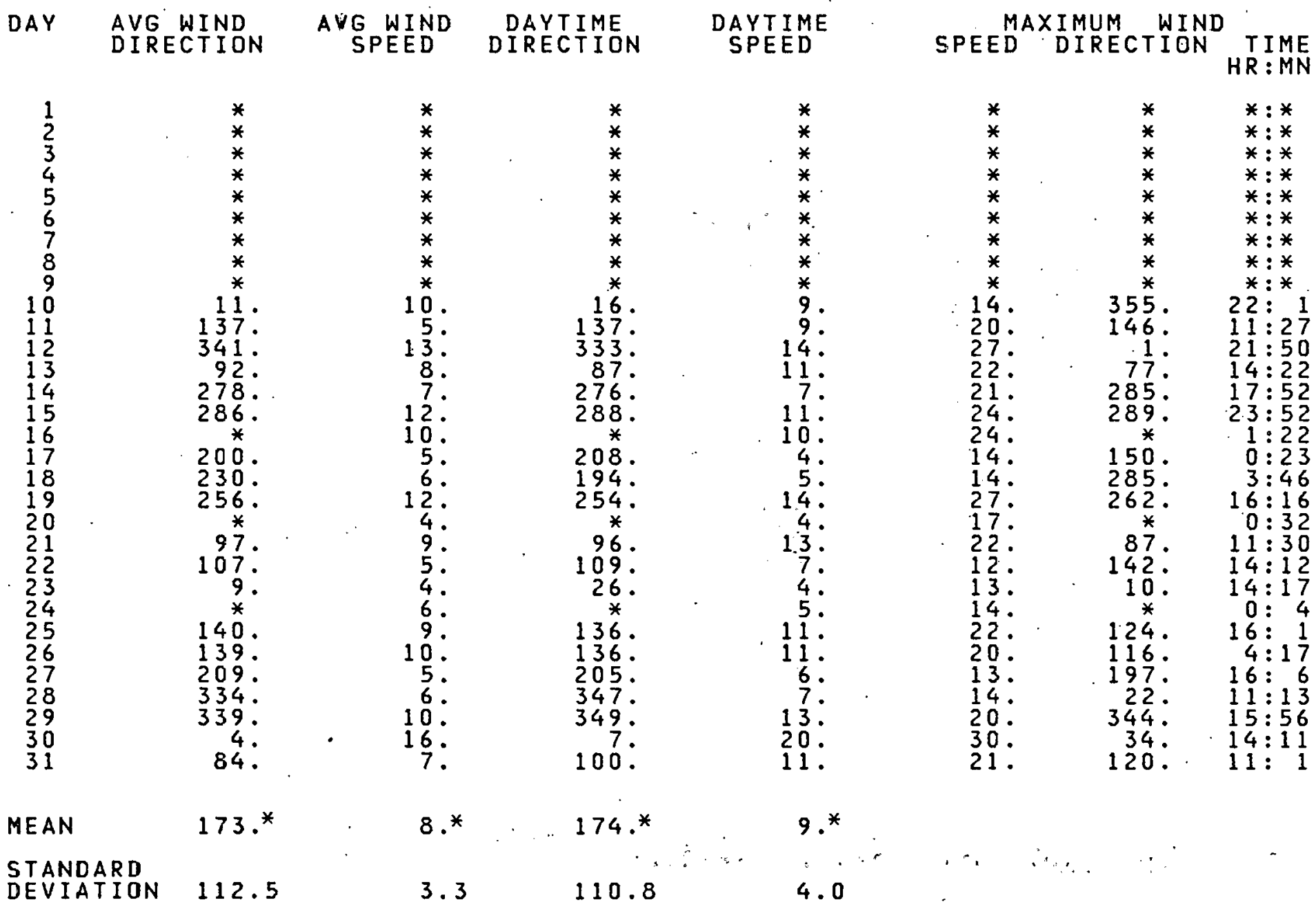


ENVIRONMENTAL SUMMARY REPORT

SITE : LO-CAL

REPORT PERIOD: $82 / 3$

SITE: LHAMPAIGN, ILLINOIS

LATITUDE: 40.05 DEG NORTH AZIMUTH: 0 DEG TIME ZONE: CENTRAL

LONGITUDE: 88.25 DEG WEST PYRANOMETERTILT: 90 DEG ALTITUDE: 750 FEET

WIND: NUMBER OF HOURS IN EACH VELOCITY INTERVAL

\begin{tabular}{|c|c|c|c|c|c|c|c|c|c|}
\hline DAY & $\begin{array}{l}0-3 \\
M P H\end{array}$ & $\begin{array}{l}4-7 . \\
\text { MPH }\end{array}$ & $\begin{array}{l}8-12 \\
M P H\end{array}$ & $\begin{array}{c}13-18 \\
M P H\end{array}$ & $\begin{array}{c}19-24 \\
M P H\end{array}$ & $\underset{\mathrm{MPH}}{25-31}$ & $\begin{array}{c}32-38 \\
\mathrm{MPH}\end{array}$ & $\begin{array}{c}A B Q V E \\
38\end{array}$ & $\begin{array}{c}\text { MISSING } \\
\text { HOURS }\end{array}$ \\
\hline $\begin{array}{r}1 \\
2 \\
3 \\
4 \\
5 \\
6 \\
7 \\
8 \\
9 \\
10 \\
10 \\
11 \\
12 \\
13 \\
14 \\
15 \\
16 \\
17 \\
18 \\
19 \\
20 \\
21 \\
22 \\
23 \\
24 \\
25 \\
26 \\
27 \\
28 \\
29 \\
30 \\
31\end{array}$ & $\begin{array}{r}0 \\
0 \\
0 \\
0 \\
0 \\
0 \\
0 \\
0 \\
0 \\
0 \\
9 \\
0 \\
8 \\
7 \\
0 \\
0 \\
10 \\
6 \\
0 \\
17 \\
2 \\
9 \\
10 \\
5 \\
1 \\
0 \\
8 \\
4 \\
0 \\
0 \\
6\end{array}$ & $\begin{array}{r}0 \\
0 \\
0 \\
0 \\
0 \\
0 \\
0 \\
0 \\
0 \\
2 \\
7 \\
1 \\
5 \\
6 \\
0 \\
6 \\
14 \\
99 \\
0 \\
7 \\
77 \\
12 \\
99 \\
19 \\
11 \\
8 \\
16 \\
16 \\
6 \\
4 \\
7\end{array}$ & $\begin{array}{r}0 \\
0 \\
0 \\
0 \\
0 \\
0 \\
0 \\
0 \\
0 \\
6 \\
4 \\
7 \\
6 \\
7 \\
15 \\
15 \\
0 \\
9 \\
16 \\
0 \\
9 \\
33 \\
5 \\
0 \\
8 \\
14 \\
0 \\
4 \\
11 \\
2 \\
9\end{array}$ & $\begin{array}{l}0 \\
0 \\
0 \\
0 \\
0 \\
0 \\
0 \\
0 \\
0 \\
0 \\
1 \\
12 \\
5 \\
4 \\
8 \\
3 \\
0 \\
0 \\
8 \\
0 \\
6 \\
0 \\
0 \\
0 \\
4 \\
2 \\
0 \\
0 \\
7 \\
8 \\
1\end{array}$ & $\begin{array}{r}0 \\
0 \\
0 \\
0 \\
0 \\
0 \\
0 \\
0 \\
0 \\
0 \\
0 \\
4 \\
0 \\
0 \\
1 \\
0 \\
0 \\
0 \\
0 \\
0 \\
0 \\
0 \\
0 \\
0 \\
0 \\
0 \\
0 \\
0 \\
0 \\
10 \\
0\end{array}$ & $\begin{array}{l}0 \\
0 \\
0 \\
0\end{array}$ & 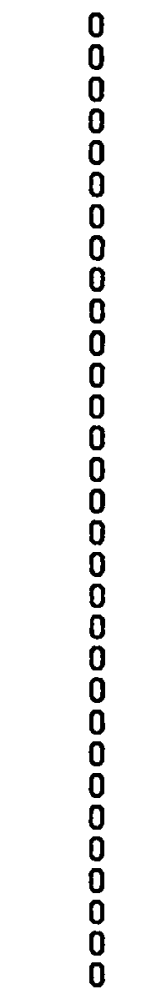 & 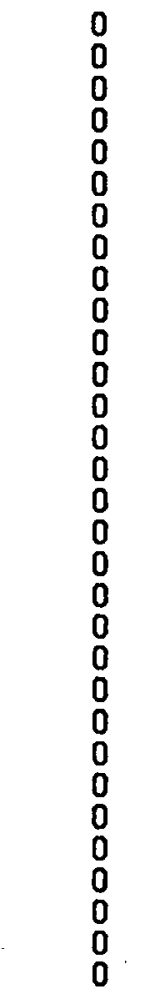 & $\begin{array}{r}24 \\
24 \\
24 \\
24 \\
24 \\
24 \\
24 \\
24 \\
24 \\
16 \\
3 \\
0 \\
0 \\
0 \\
0 \\
0 \\
0 \\
0 \\
0 \\
0 \\
0 \\
0 \\
0 \\
0 \\
0 \\
0 \\
0 \\
0 \\
0 \\
0 \\
1\end{array}$ \\
\hline AVERAGE & 3.3 & 5.5 & 4.8 & 2.2 & 0.5 & 0.0 & 0.0 & 0.0 & 7.6 \\
\hline TOTAL & 102 & 172 & 150 & 69 & 15 & 0 & 0 & 0 & 236 \\
\hline
\end{tabular}


SITE: LO-CAL

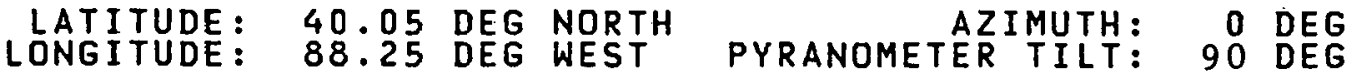
RELATIVE HUMIDITY IN PER CENT

\begin{tabular}{|c|c|}
\hline DAY & $\begin{array}{l}\text { RELATIVE } \\
\text { HUMID I YY }\end{array}$ \\
\hline $\begin{array}{r}1 \\
2 \\
3 \\
4 \\
5 \\
6 \\
7 \\
8 \\
9 \\
10 \\
11 \\
12 \\
123 \\
14 \\
15 \\
16 \\
17 \\
18 \\
19 \\
20 \\
21 \\
22 \\
23 \\
24 \\
25 \\
26 \\
27 \\
28 \\
29 \\
30 \\
31\end{array}$ & $\begin{array}{l}* \\
* \\
* \\
* \\
* \\
* \\
* \\
* \\
* \\
* \\
81 . \\
74 . \\
81 . \\
54 . \\
56 . \\
84 . \\
77 . \\
70 . \\
74 . \\
84 . \\
84 . \\
65 . \\
56 . \\
59 . \\
57 . \\
61 . \\
48 . \\
46 . \\
53 . \\
49 . \\
69 . \\
41 .\end{array}$ \\
\hline MEAN & $65 .^{*}$ \\
\hline
\end{tabular}

DEVIATI ON 14.

$\begin{array}{cc}\text { DAYTIME } & \text { NIGHTTIM } \\ \text { REL HUM } & \text { REL HUM } \\ & \\ * & * \\ * & * \\ * & * \\ * & * \\ * & * \\ * & * \\ * & * \\ * & * \\ * & * \\ 80 . & 86 . \\ 67 . & 77 . \\ 34 . & 78 . \\ 44 . & 63 . \\ 45: & 67 . \\ 34 . & 84 . \\ 71 . & 82 . \\ 57 . & 73 . \\ 73 . & 76 . \\ 36 . & 83 . \\ 32 . & 85 . \\ 57 . & 73 . \\ 50 . & 62 . \\ 51 . & 67 . \\ 52 . & 61 . \\ 58 . & 64 . \\ 42 . & 53 . \\ 37 . & 54 . \\ 46: & 60 . \\ 38 . & 58 . \\ 68 . & 70 . \\ 27 . & 54 . \\ & \\ 59 . & 70 . \\ & \\ 18 . & 11 . \\ 18 . & \end{array}$

REPORT PERIOD: $82 / 3$ SITE NUMBER: 223

TIME ZONE: CENTRAL ALTITUDE: 750 FEET 
ZONE 8

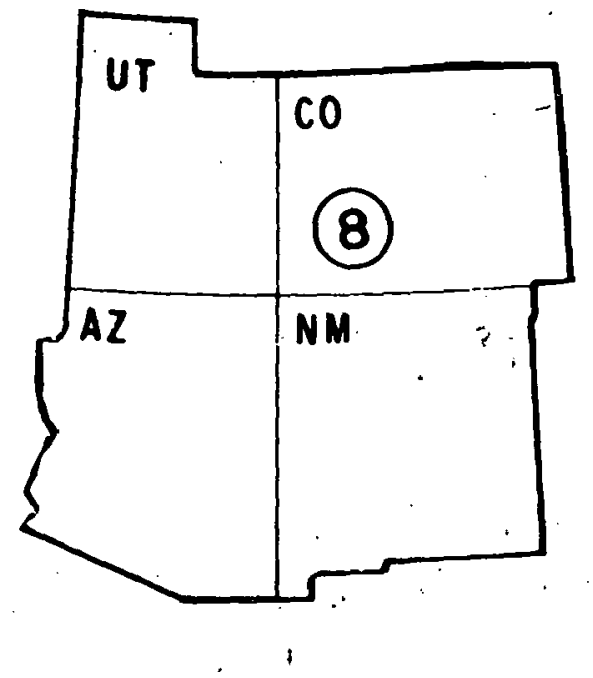


ENVIRONMENTAL SUMMARY REPORT

SITE: CROWN REALTY \#I
LAKEWOOD, COLORADO

LATITUDE: $\quad 39.41$ DEG NORTH

LONGITUDE: 105.00 DEG WEST
PYRANOMETER AZILT: $\quad 50$ DEG INSOLATION
REPORT PERIOD: $82 / 3$
SITE NUMBER: 88

TIME ZONE: MOUNTAIN

ALTITUDE : $\quad 5280$ FEET

DAY

$\begin{array}{ccc}\text { TOTAL } & \text { TOTAL DIFF } \\ \text { INSOLATION TOTAL DIR } \\ \text { BTU/FT2 }\end{array}$

MAXIMUM TOTAL INSOLATION
VALUE TIME
BTUYHR/FT2 HR:MN

EXTRATERRESTRIAL TTL INS BTU/FT INSOLATION
BTU/FT2

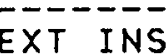

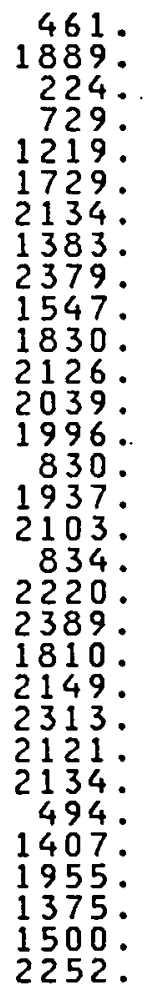

N.A.

$N \cdot A$.

N.A.

N.A.

N.A.

N.A.

N.A.

N.A.

N.A.

N.A.

N.A.

N.A.

N.A.

$N \cdot A$

N.A.

$N \cdot A$.

N.A.

N.A.

$N \cdot A$.

N.A.

N.A.

N.A.

N.A.

N.A.

N.A.

N.A.

N.A.

N.A.

$N \cdot A$.

N.A.

N.A.

$N \cdot A$.

$N \cdot A$.

N.A.

N.A.

N.A.

N.A.

N.A.

$N \cdot A$.

$N \cdot A$.

N.A.

N.A.

N.A.

N.A.

N.A.

N.A.

N.A.

$N \cdot A$.

N.A.

N.A.

N.A.

N.A.

$N \cdot A$.

N.A.

N.A.

186.

9:53

3329 .

3329.

$169.15: 23$

$355^{\circ}$.

$15: 23$

$355 . \quad 12: 32$

$3620^{\circ} \quad 12: 48$

382 .

$252 \cdot 12: 22$

$293^{\circ} \quad 14: 25$

$334 . \quad 14: 25$

338 . $11: 28$

327 .

$11: 55$

$369^{\circ}$

$11: 51$

$11: 22$

$327 . \quad 12: 6$

$193 \circ \quad 12: 6$

372 .

$12: 4 \frac{1}{5}$

$12: 5$

$11: 54$

$11: 31$

327 .

$11: 59$

324 .

$11: 56$

314.

$11: 59$

193.

$11: 53$

382 .

$12: 31$

$12: 27$

$355^{\circ}$.

12:11

314 .

1 $1: 18$

N.A.

N.A.

3327 .

3326 .

3319.

उदा?

3309 .

3307 .

3303 .

3292 .

3288.

3279.

3268 .

3259 .

3253 .

3239 .

3228 .

3215 .

3205 .

$3180^{\circ}$.

3167 .

3156 .

3142.

3116 .

PERCENT

MEAN

1661 .

N.A.

N.A.

3256 .

66.

14.

7.

22 .

52 .

64.

72 .

47.

64.

62 .

61.

59.

64.

26 .

68.

$56^{\circ}$.

67.

72 .

66.

67.

16.

44.

62.

48.

72 .

STANDARD

DEVIATION 620 . 
ENVIRONMENTAL SUMMARY REPORT

SITE: CROWN REALTY \#I
LAKEWOOD, COLORADO

LATITUDE: $\quad 39.41$ DEG NORTH PYRANOMETEIMUTH: 0 DEG

\section{LONGITUDE: 105.00 DEG WEST PYRANOMETER TILT: 55
TEMPERATURES IN DEGREES F}

\section{REPORT PERIOD: $82 / 3$
SITE NUMBER: 88}

TIME ZONE : MOUNTAIN

\begin{tabular}{|c|c|c|c|c|}
\hline DAY & $\begin{array}{l}\text { AVERAGE } \\
\text { TEMP }\end{array}$ & $\begin{array}{c}\text { DAYTIME } \\
\text { TEMP. }\end{array}$ & NIGHTT IME & $\begin{array}{c}\text { MAX TEMPERATURE } \\
\text { VALUE TIME } \\
\text { HR:MN }\end{array}$ \\
\hline $\begin{array}{r}1 \\
2 \\
3 \\
4 \\
5 \\
6 \\
7 \\
8 \\
9 \\
10 \\
1.1 \\
12 \\
13 \\
14 \\
15 \\
16 \\
17 \\
18 \\
19 \\
20 \\
21 \\
22 \\
23 \\
24 \\
25 \\
26 \\
27 \\
28 \\
29 \\
30 \\
31\end{array}$ & 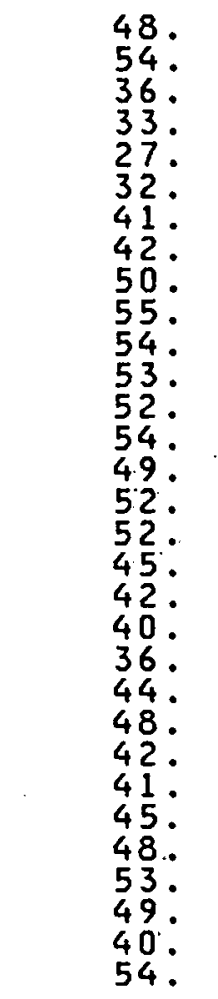 & 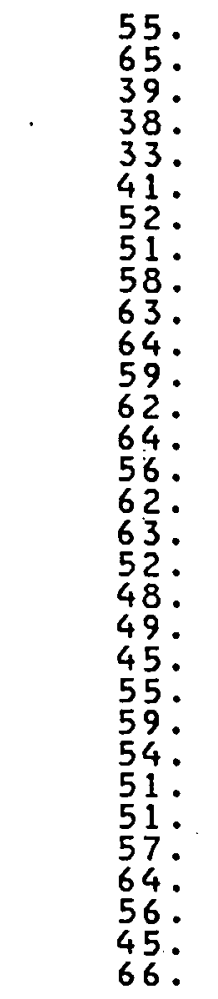 & 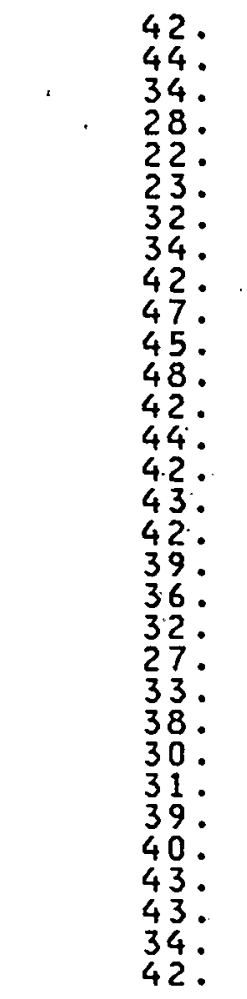 & 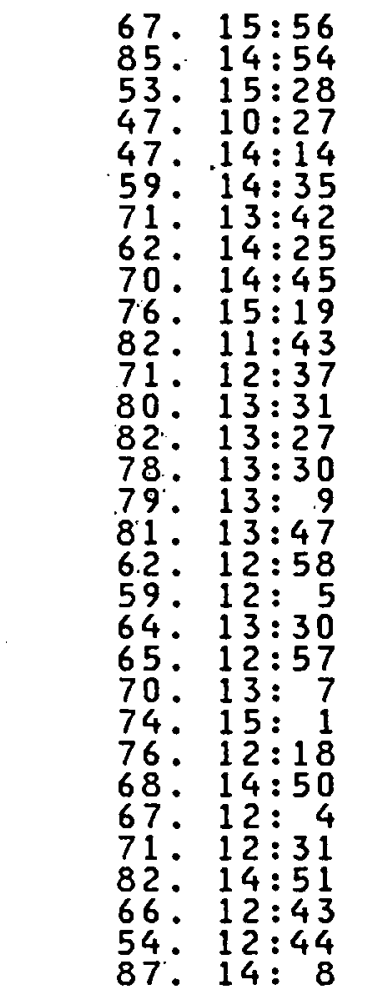 \\
\hline MEAN & 45. & 54. & 37. & \\
\hline DEV: & 7.5 & 8.7 & 6.9 & \\
\hline
\end{tabular}

\section{MIN TEMPERATURE INLET WATER
VALUE TIME
HR:MN}

$33.4: 28$

$35 \cdot 23: 58$

$32 \cdot 23: 28$

$21 \cdot 23: 26$

17. $6: 14$

21. $6: 14$

28. $6: 46$

29. $3: 45$

$40.23: 56$

$33 . \quad 5: 37$

$38 \cdot 23: 55$

34 . $5: 25$

34. $5: 0$

31 . $5: 30$

32 . $5: 46$

$34 . \quad 5: 10$

$33 \cdot 23: 54$

$17: 6: 5$

21. $5: 50$

26. 5:50

$23: 23: 24$

$23.2: 31$

$30 \cdot \quad 3: 1 \frac{1}{34}$

$34 \cdot 23: 59$

$37: 23: 23$

$30.5: 43$

N.A.

N.A.

N.A.

N.A.

N.A.

N.A

N.A.

N.A.

N.A.

N.A.

N.A

N.A.

N.A.

N.A

N.A.

N.A.

N.A.

N.A

N.A.

N.A.

N.A.

N.A.

N.A.

N.A.

N.A.

N.A

N.A.

N. A . 
ENVIRONMENTAL SUMMARY REPORT

SITE: CUSHING HOME

LATITUDE: $\quad 41.50$ DEG NORTH LONGITUDE: 111.80 DEG WEST
PYRANOMETER AZIMUTH: $\quad-5$ DEG INSOLATION
REPORT PERIOD: $82 / 3$
SITE NUMBER: 240

TIME ZONE : MOUNTAIN
ALTITUDE :
DAY $\begin{array}{cc}\text { TOTAL } & \text { TOTAL DIFF TOTAL DIR } \\ \text { INSOLATION } & \text { INSOLATION INSOLATION } \\ \text { BTU/FT2 } & \text { BTU/FT2 }\end{array}$

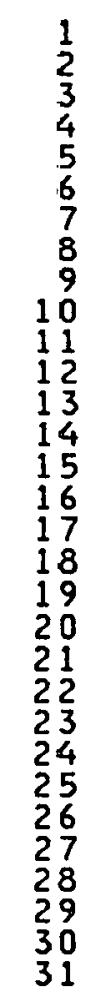

$$
\begin{aligned}
& \begin{array}{l}
157 . \\
652 .
\end{array} \\
& 41 \frac{1}{4} \text {. } \\
& 1613 . \\
& 2133 \\
& 1718 \text {. } \\
& 1390 \text {. } \\
& 2144 \text {. } \\
& 328 \text {. } \\
& 2362
\end{aligned}
$$

778 .
N.A.

$N \cdot A$.

N.A.

N.A.

N.A.

$N \cdot A$.

N.A.

N.A.

N.A.

N.A.

N.A.

N.A.

N.A.

N.A.

$N \cdot A$.

N.A.

N.A.

N.A.

N.A.

$N \cdot A$.

$N \cdot A \cdot$

N.A.

N.A.

N.A.

N.A.

N.A.

N.A.

N.A.

N.A.

N.A.

$\begin{array}{ccc}\text { MAXIMUM TOTAL } & \text { EXTRATERRESTRIAL } & \text { TTL INS } \\ \text { INSOLATION } & \text { INSOLATION } & \text { INSTI } \\ \text { VALUE TIME } & \text { BTU/FT2 } & \text { EXT INS } \\ \text { BTU/HR/FT2 HR:MN } & & \text { PERCENT }\end{array}$

54. $12: 29$

$1 \frac{1}{3}: 37$

$13: 37$

$16: 1$

$13: 12$

$12: 12$

$13: 3 \frac{1}{3}$

$12 .: 30$

$12: 5$

$9: 50$

$12: 56$

$11: 57$

$13: 44$

$12: 36$

$12: 27$

$11: 26$

$13: 39$

$13: 1$

$12: 37$

$13: 12$

$12: 52$

$13: 29$

$11: 36$

$12: 11$

$13: 33$

$13: 39$

$\begin{array}{ll}319 \circ & 13: 52 \\ 134 . & 14: 27\end{array}$

3325.

3328
3328

3330 .

3326

3331.

3328

3325 .

3326 .

3326 .

3322

3315 .

3317 .

3304 .

3300 .

3295 .

3291.

3285.

3276 .

3274.

3258 .

3252 .

3245 .

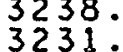

3220

3216 .

$20^{\circ}$ :

$20^{\circ}$

14.

49.

$52^{\circ}$.

52.
42.
64.

64.

23 .

$71^{\circ}$.

69.

10 .

18

8 .

20.

46.

$71^{\circ}$.

64.

67.

69.

48 .

54.

26 .

3295.

37. 
ENVIRONMENTAL SUMMARY REPORT

SITE: CUSHING HOME

REPORT PERIOD: 8213
SITE NUMBER: 240

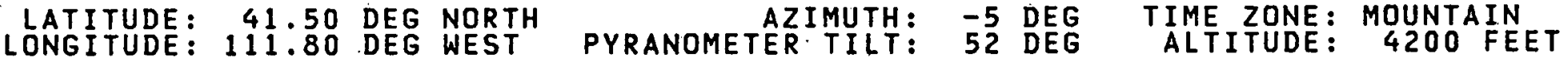
TEMPERATURES IN DEGREES F

\begin{tabular}{|c|c|c|c|c|c|c|}
\hline DAY & $\begin{array}{l}\text { AVERAGE } \\
\text { TEMP }\end{array}$ & $\begin{array}{c}\text { DAYTIME } \\
\text { TEMP }\end{array}$ & $\underset{\text { TEMP. }}{\text { NIGHTIME }}$ & $\begin{array}{l}\text { MAX TEMPERATURE } \\
\text { VALUE TIME } \\
\text { HR:MN }\end{array}$ & $\begin{array}{c}\text { MIN TEMPERATURE } \\
\text { VALUE TIME } \\
\text { HR:MN }\end{array}$ & $\begin{array}{l}\text { INLET WATER } \\
\text { TEMPERATURE }\end{array}$ \\
\hline
\end{tabular}

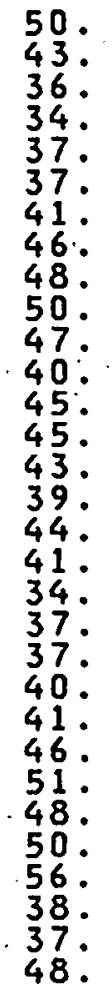

54.47.

45.. $\quad 42$.

$38 . \quad 30$.

43 .

44.

$54^{\circ}$.

54 .

59 .

51.

59.

49 .

$43^{\circ}$.

53 .

44.

37.

49 .

54.

53.

68.

50.

57.

38 .

40.
51.

MEAN

43.

49.

5.7

7.2

37.
58. $12: 39$

$55 \cdot 14: 57$
$46 \cdot 13: 58$

47. $16: 12$

$63.9: 17$

$56 \cdot 11: 36$

64. $11: 15$

$67 \% 15 \vdots 15$

$77^{\circ}: 12: 32$

54. $9: 45$

66. $9: 49$

76. $10: 32$

56. $13: 4$

$50: 11: 32$

57. 13:31

$54: 11: 26$

$48.14: 5$

63. $9: 17$

$64 \cdot 10: 18$

71. $10: 49$

75. 12:35

70. $13: 1$

55. $15: 32$

70. $10: 13$

66. $15: 25$

$40.12: 29$

57: $15: 42$
$41 ., 1: \frac{12}{36}$

$28: 22: 3$

$28.22: 33$

26. $23: 41$

24. $5: 28$

30. $0: 2$

32. $1: 48$

29. $3: 15$

$33: 23: 53$

21. $6: 32$

22. $6: 48$

30 . $1: 17$

36 . $22: 42$

32 . $20: 26$

$34.23: 7$

$32 \cdot 22: 38$

$30.20: 8$

$17 \cdot 23: 57$

19. $4: 58$

20. 6:16

27. $0: 25$

$27.4: 14$

$38.23: 38$

35. $1: 3$

$47: \quad 6: 35$

$30^{\circ}$

37 . $4: 17$

6.6
65.

61.

60 .

61 .

64.

64.

62.

65.

$666^{\circ}$

65 .

64.

64.

64.

$63^{\circ}$.

63.

65.

65.

66.

65.

69.

66.

60 .

66.

64.

63.

64:

2.0 
ENVIRONMENTAL SUMMARY REPORT

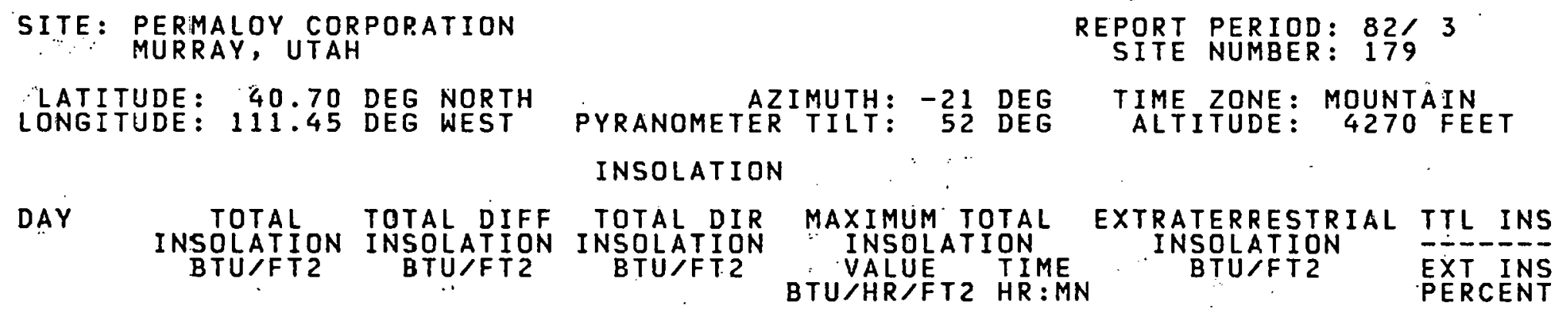

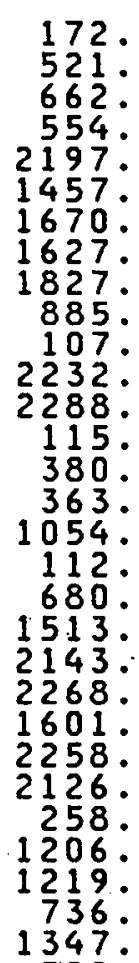

N.A.

N.A.

N.A:

590 :

N.A.
N.A.
N.A.
N.A.
N.A.
N.A.
N.A.
N.A.
N.A.
N.A.
N.A.
N.A.
N.A.
N.A.
N.A.
N.A.
N.A.
N.A.
N.A.
N.A.
N.A.
N.A.
N.A.
N.A.
N.A.
N.A.
N.A.
N.A.
N.A.
N.A.
N.A.

104.

$9: 31$

3184 .

$354^{\circ} \quad 13: 51$

354.

$12: 34$

$13: 36$

$12: 21$

N.A.

N.A.

N.A.

N.A.

N.A.

N.A.

N.A.

N.A.

N.A:

N.A.

N.A.

N.A.

N.A.

$N: A^{\prime}$.

N.A.

N.A.

N.A.

N.A.

N.A.

N.A.

N.A.

370 :

348 . $12: 32$

$14: 19$

$12: 32$

$12: 45$

$12: 41$

$12: 35$

$15: 29$

$13: 22$

$11: 28$

$14: 13$

$13: 19$

$12: 15$

$12: 49$

$1.2: 44$

$13: 37$

$12: 45$

$12: 42$

$12: 20$

$11: 53$

$14: 27$

$11: 36$
$9: 19$

N. A .

N.A.

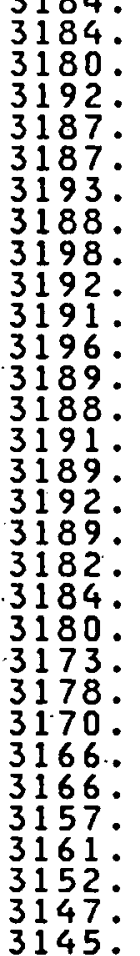

5.

MEAN

1167.

N. A .

N.A. 
ENVIRONMENTAL SUMMARY REPORT

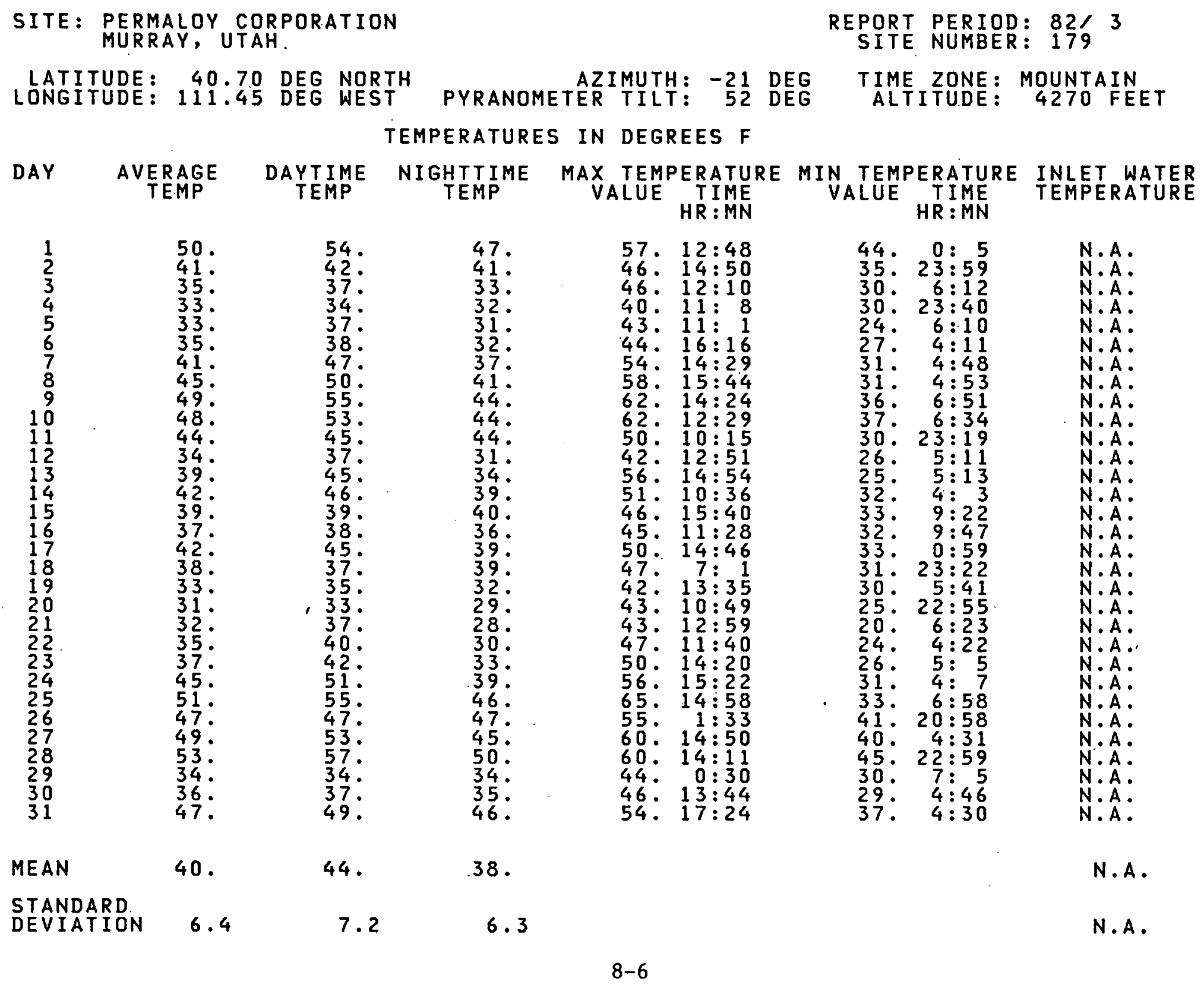


ENVIRONMENTAL SUMMARY REPORT

SITE: PERMALOY CORPORATION

REPORT PERIOD: $82 / 3$
SITE NUMBER: 179.

LATITUDE: 40.70 DEG NORTH PYRANOMETER TILT:
LONGITUDE: 111.45 DEG WEST DEG PIME

WIND: SPEED IN MPH-DIRECTION IN DEGREES

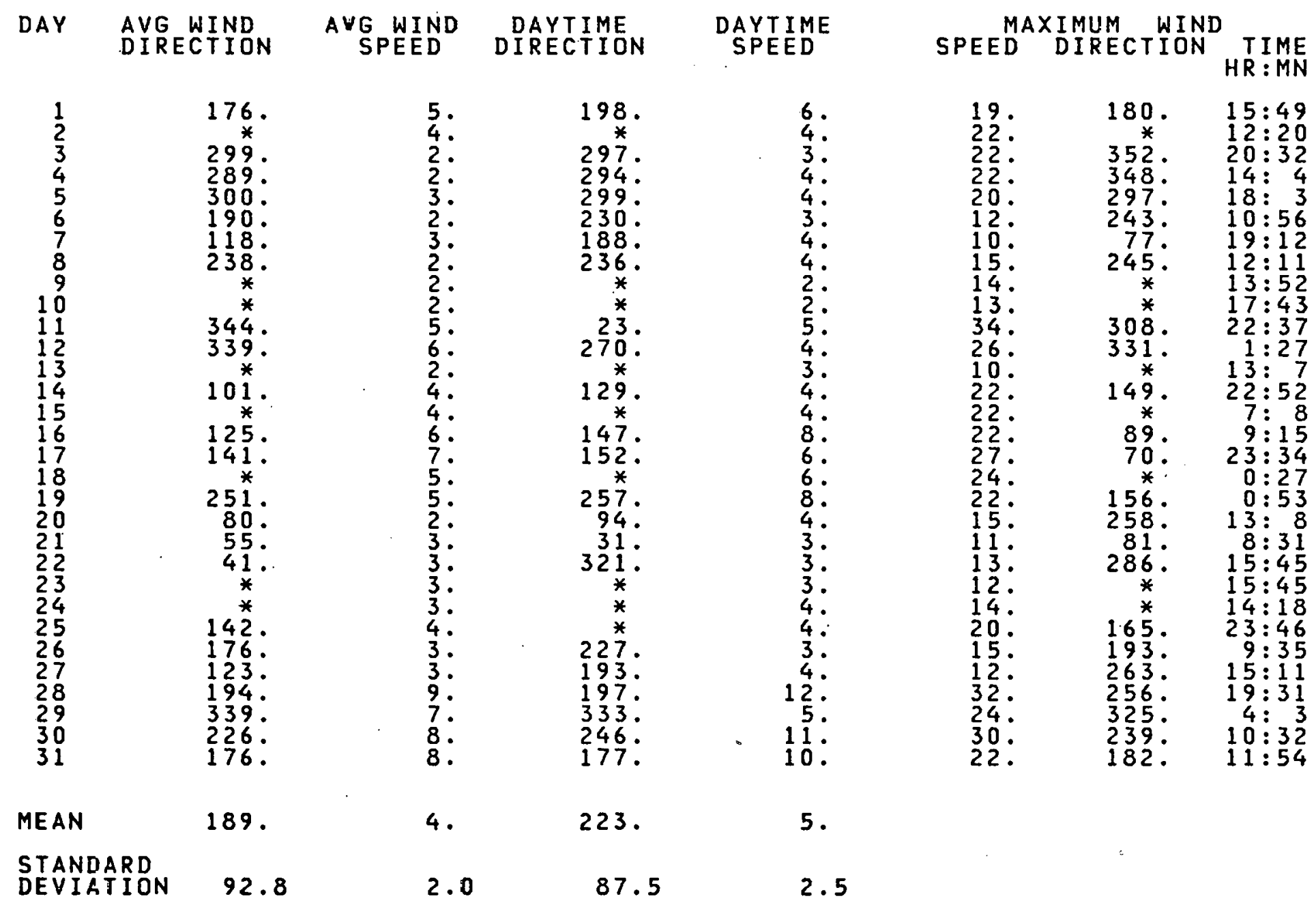




\section{ENVIRONMENTAL SUMMARY REPORT}

SITE: PERMALOY CORPORATION

REPORT PERIOD: $82 / 3$

LATITUDE: 40.70 DEG NORTH AZIMUTH: -21 DEG TIME ZONE: MOUNTAIN LONGITUDE: 111.45 DEG WEST PYRANOMETER TILT: 52 DEG ALTITUDE: 4270 FEET

WIND: NUMBER OF HOURS IN EACH VELOCITTY INTERVAL

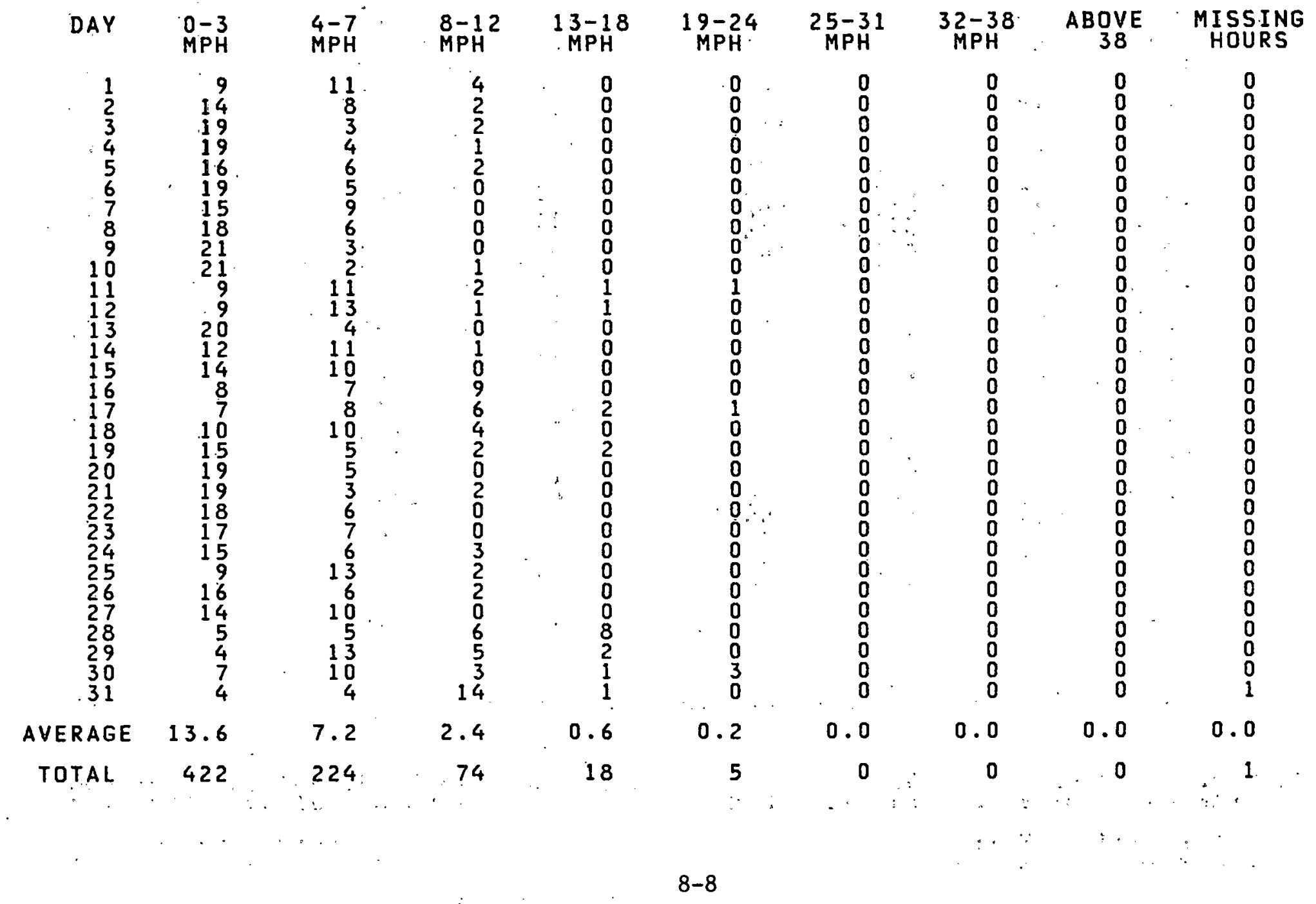


ENVIRONMENTAL SUMMARY REPORT

SITE: PERMALOY CORPORATION

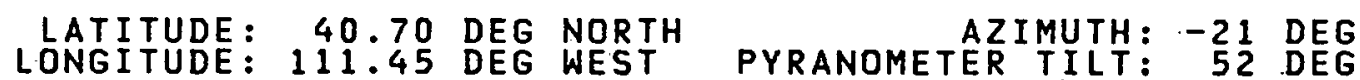
RELATIVE HUMIDITY IN PER CENT

DAY RELATIVE DAYTIME NIGHTTIME MAXIMUM HUMIDITY HUMIDITY. REL HUM REL. HUM V VALUE TIME
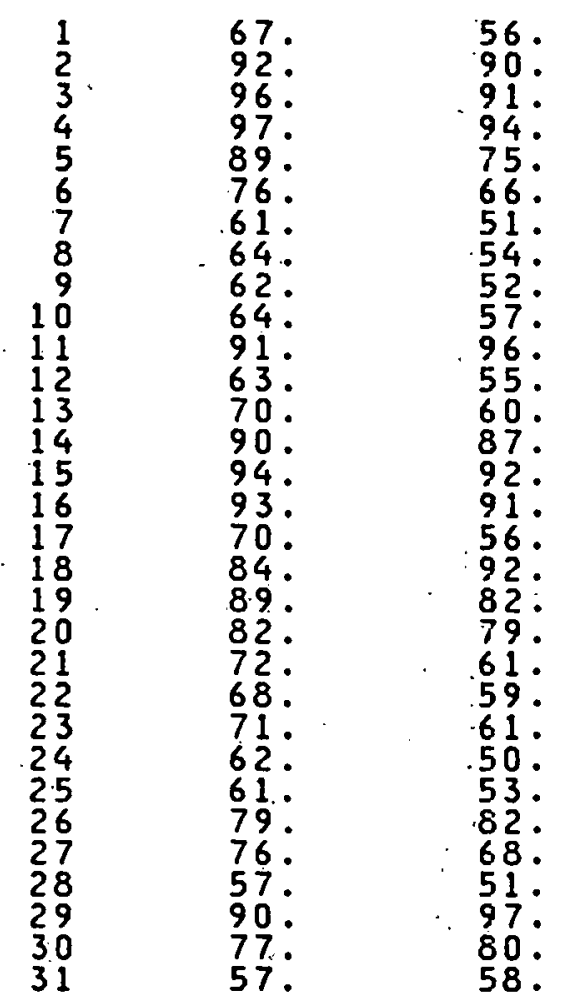

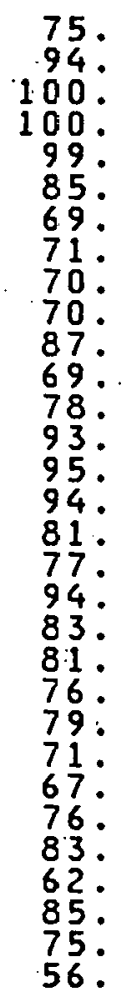

95

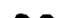

$22: 3$

$100: \quad 6: 8$

$100 . \quad 0: 10$

100 . $0: 0$

$100 . \quad 0: 2$

$100 . \quad 0: 27$

86. $\quad 3: 33$

$92 . \quad 5: 4$

$87 . \quad 7: 6$

88 .

100 .

100 .

193.

100.

100 .

100 .

100 .

100 .

.98.

94.

88.

.95.

100 .

100 .

100 .

73.

100 .

$5: 46$ $0: 2$

$6: 43$

$12: 46$

$0: 28$

$3: 51$

$0: 53$

$11: 49$

$0: 5$

$6: 33$

$4: 17$

$2: 25$

$6: 29$

$15: 48$

$0: 4$

$6: 55$

$2: 43$

68 .

$3: 21$
$6: 55$

REPORT PERIOD: $82 / 3$
SITE NUMBER: 179

TIME ZONE: $\begin{aligned} & \text { MOUNTAIN } \\ & \text { ALTITUDE : }\end{aligned}$

MINIMUM HUMIDITY

VALUE TIME

50. $\quad 12: 48$

$68 . \quad 16: 47$

$64.13: 14$

$56 . \quad 13: 6$

52. $14: 29$

38. $14: 51$

$39 . \quad 15: 28$

40. 14:24

49. 15: $\quad 0$

47. $11: 15$

$50 . \quad 11: 31$

67 . $15: 56$

$72 . \quad 13: 25$

$47.14: 24$

61. $13: 24$

64. $18: 17$

49. $16: 1$

$44^{\circ} \quad 14: 36$

$46^{\circ} \quad 14: 36$

42 . $\quad 16: 53$

$36 . \quad 15: 25$

48. $\quad 16: 36$

$42 . \quad 17.50$

$64.253: 47$

$640^{\circ} \quad 23: 47$

$\begin{array}{ll}50 \cdot \cdot & 16: 8 \\ 48 . & 21: 8\end{array}$

MEAN 76.

71.

80.

STANDARD

DEVIATION 13.

17.

12. 
ENVIRONMENTAL SUMMARY REPORT

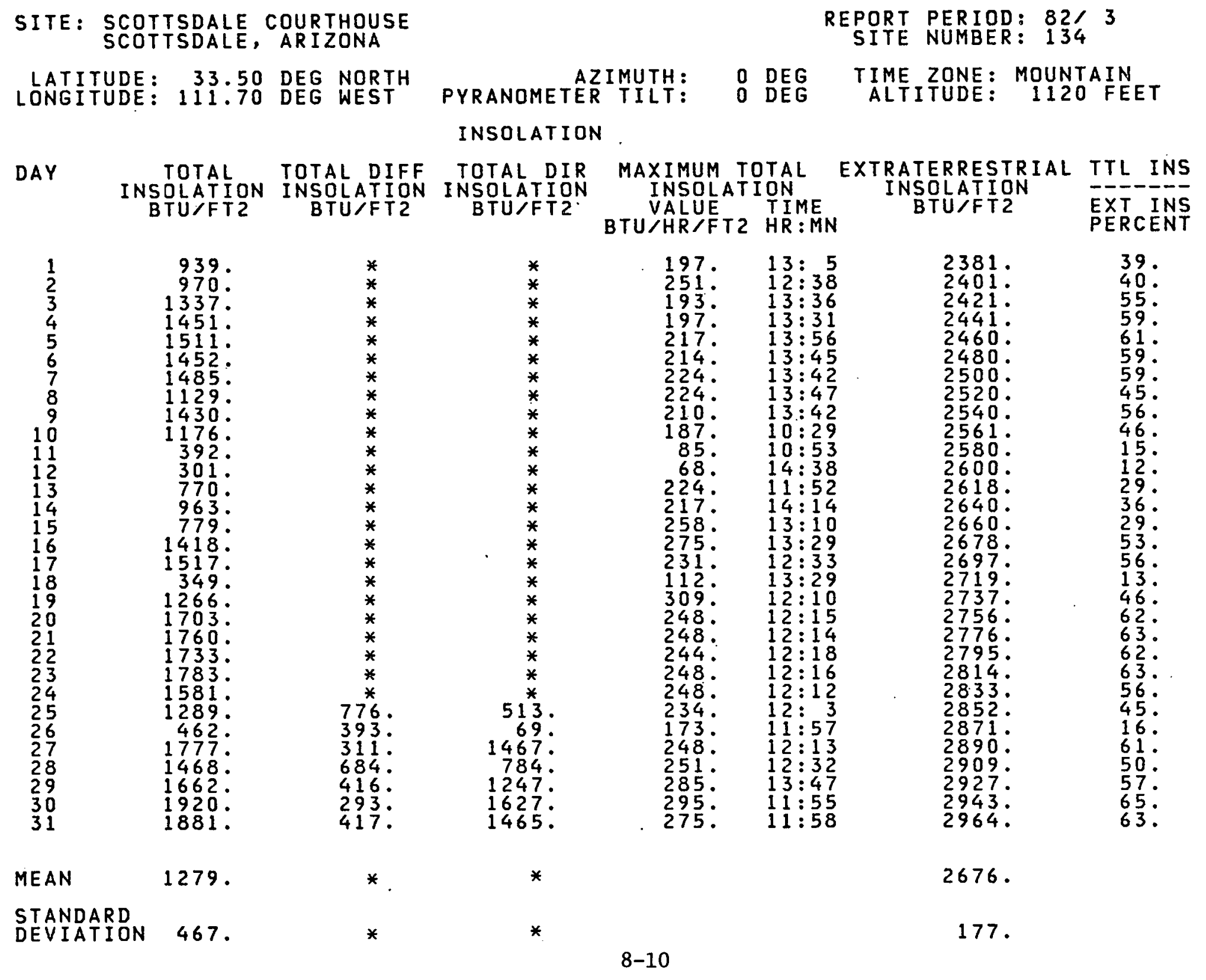


ENVIRONMENTAL SUMMARY REPORT

SITE: SCOTTSDALE COURTHOUSE LOATITJDE: $\begin{array}{r}33.50 \text { DEG NORTH } \\ \text { LONGIUDE: } 111.70 \text { DEG WEST }\end{array}$

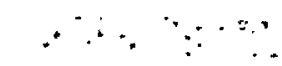

PYRANOMETER TILT:

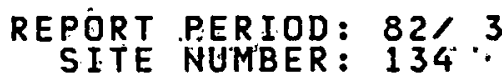

$\begin{array}{lll}0 & \text { DEG } & \text { TIME ZONE: MOUNTAIN } \\ 0 & \text { DEG ALTITUDE: } & \text { IIZO FEET }\end{array}$

TEMPERATURES IN DEGREES F

\begin{tabular}{|c|c|c|c|c|c|c|}
\hline DAY & $\begin{array}{c}\text { AVERAGE } \\
\text { TEMP }\end{array}$ & $\begin{array}{c}\text { DAYTIME } \\
\text { TEYMP }\end{array}$ & NIGHTTIME & $\begin{array}{c}\text { MAX TEMPERATURE } \\
\text { VALUE TIME } \\
\text { HR:MN }\end{array}$ & $\begin{array}{c}\text { MIN TEMPERATURE } \\
\text { VALUE TIME } \\
\text { HR:MN }\end{array}$ & $\begin{array}{l}\text { INLET WATER } \\
\text { TEMPERATURE }\end{array}$ \\
\hline $\begin{array}{l}1 \\
2 \\
3 \\
4 \\
5 \\
6 \\
7 \\
8 \\
9 \\
10 \\
11 \\
12 \\
13 \\
14 \\
15 \\
16 \\
17 \\
18 \\
19 \\
20 \\
21 \\
22 \\
23 \\
24 \\
25 \\
26 \\
27 \\
28 \\
29 \\
30 \\
31\end{array}$ & 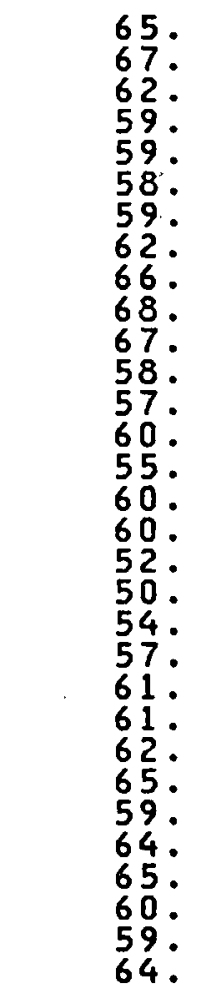 & $\begin{array}{l}71 . \\
70: \\
66: \\
66: \\
64: \\
64: \\
68: \\
69: \\
75: \\
75: \\
70: \\
58: \\
59: \\
64: \\
59: \\
65: \\
65: \\
54: \\
56: \\
61: \\
64: \\
69: \\
69: \\
69: \\
70: \\
57: \\
70: \\
71: \\
62: \\
65: \\
72 .\end{array}$ & 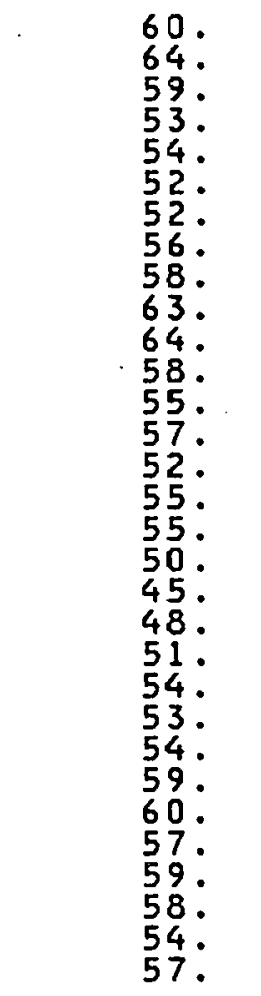 & 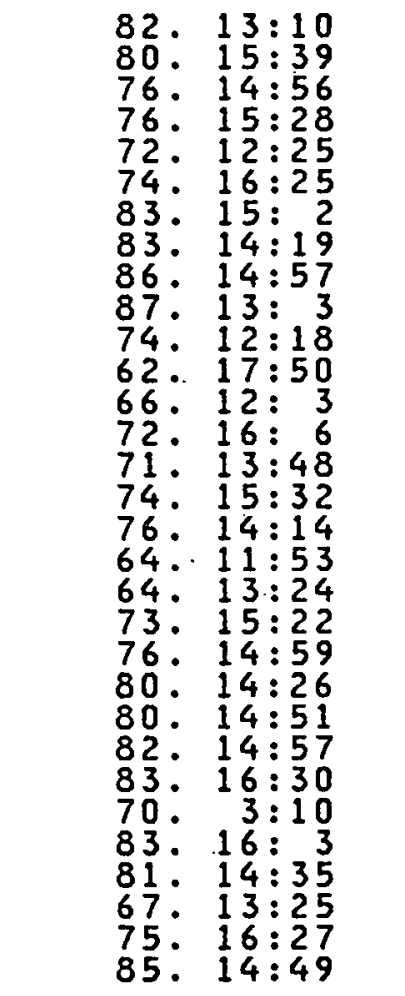 & 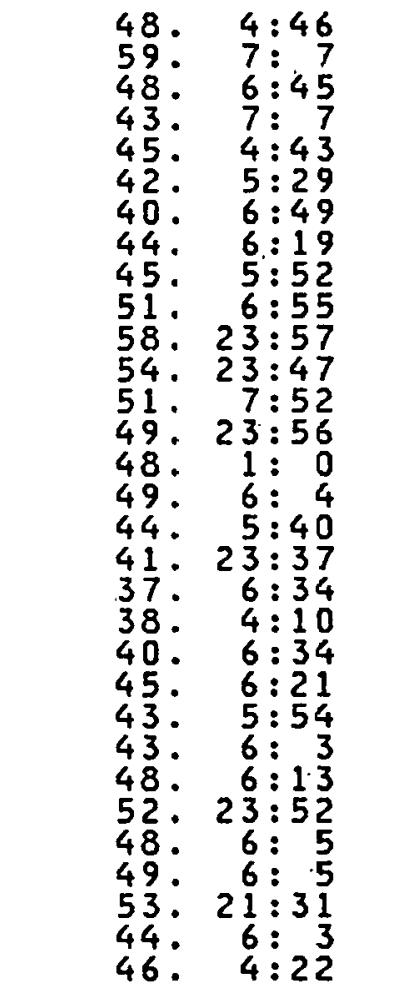 & 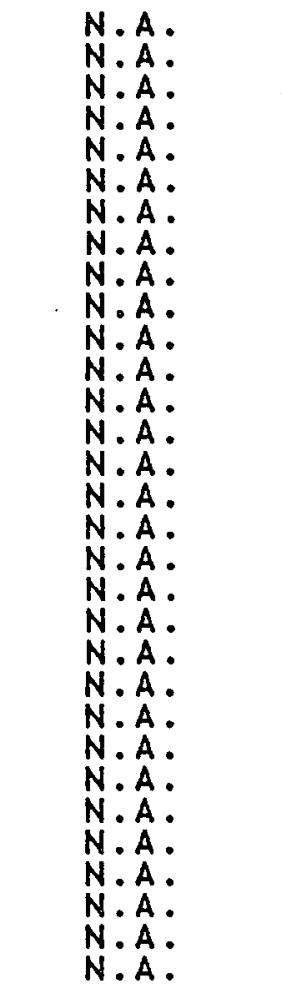 \\
\hline MEAN & 61. & 66. & 56. & $\cdot \cdot$ & $\because$ & N.A. \\
\hline $\mathrm{V}$ & .3 & 5.4 & .4 & & & N.A. \\
\hline
\end{tabular}


ENVIRONMENTAL SUMMARY REPORT

SITE: TAOS STA.TE OFFICE BUILDING

LLATITUDE: $\begin{array}{r}36.50 \\ \text { LONGITUDE: }\end{array}$ PYRANOMETER TILT:

INSOLATION
REPORT PERIOD: $82 / 3$
SITE NUMBER: 186

O DEG TIME ZONE: MOUNTAIN O DEG ALTITUDE: 6980 FEET
DAY $\begin{array}{cc}\text { TOTAL } & \text { TOTAL 9ODEG } \\ \text { HORIZONTAL INSOLATION IN } \\ \text { BTU/FT2 }\end{array}$

TOTAL DIR INSOLATION BTU/FT2 MAXIMUM TOTAL
EXTRATERRESTRIAL
INSOLATION
VALUE INSOLATION
BTUAHR/FT2 HR:MN

N.A.

$\begin{array}{lll}1 & 1506 . & 1983 . \\ 2 & 1153 . & 984 .\end{array}$

1371 .

$850^{\circ}$.

1633.

1183

1763 .

1318 .

1142 .

747.

433.

726 .

1906 .

2014 .

1564 .

1907 .

$1943^{\circ}$.

1806 .

2045.

1987.

$488^{\circ}$.

2069.

1963.

1142.

$2198^{\circ}$.

1413.

551 .

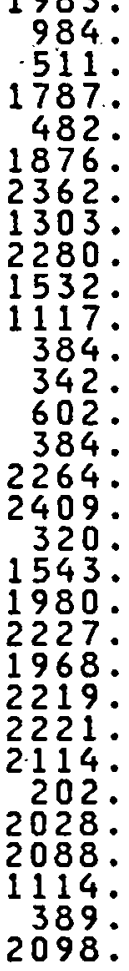

2098 .

1455.

777.

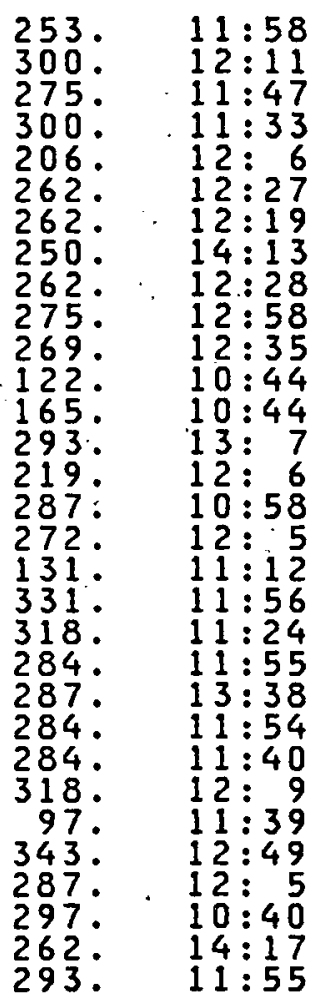

N.A.

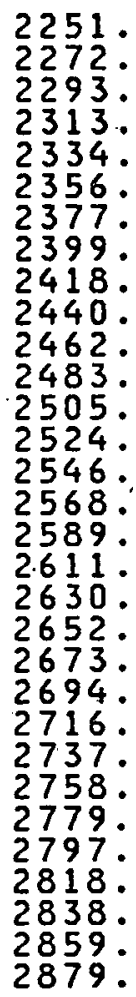

EXT-OTS

EXT INS

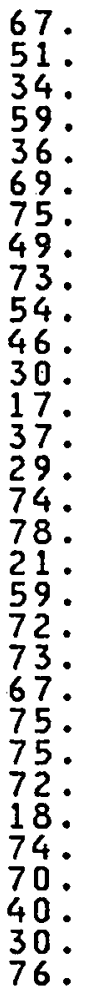

2567. 
ENVIRONMENTAL SUMMARY REPORT

SITE: TAOS STATE OFFICE BUILDING

REPORT PERIOD: $82 / 3$
SITE NUMBER: 186

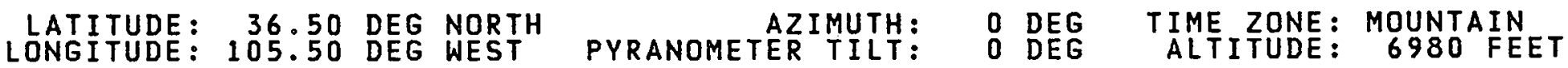

WIND: SPEED IN MPH-DIRECTION IN DEGREES $\begin{array}{ll}\text { DAY } & \text { AVG WIND } \\ \text { DIRECTION }\end{array}$

\section{AVG WIND DAYTIME}

356.

$349^{\circ}$.

348 .

224 .

$3299^{\circ}$

282

$327^{\circ}$

352.

348 .

226 .

$355^{\circ}$.

338 .

306 .

358 .

$35 \frac{1}{3}$.

332

$355:$

.

53.

341 .

349 .

$357^{\circ}$.

MEAN

276 .

STANDARD

DEVIATION

125.2

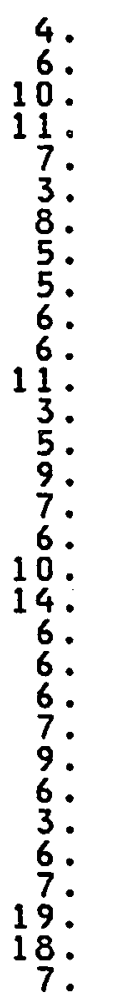

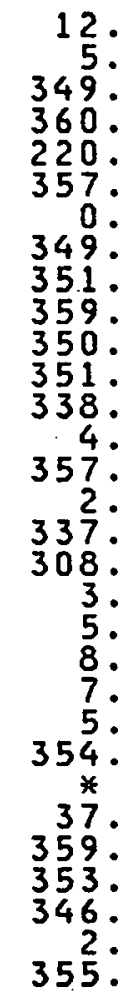

SPEED

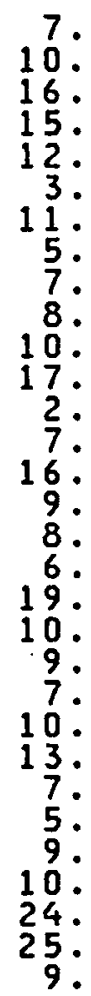

208 .

3.8

168.6

10
MAXIMUM WIND SPEED DIRECTION TIME

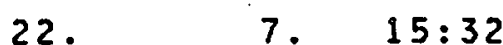

23. $\quad 345^{\circ} \quad 12: 38$

$32 . \quad 347 . \quad 13: 18$

12. $11: 49$

$36.206 .29: 10$

13.0 $\quad 349^{\circ} \quad 14: 51$

$14: 24$

25. $351 . \quad 15: 46$

$38^{\circ} \quad 13: 20$

$338 . \quad 16: 9$

10. $222 . \quad 6: 2$

$21^{\circ} \quad 347 . \quad 12: 51$

$40.015 .15: 55$

8. $\quad 14: 58$

$331 . \quad 17: 20$

2. $14: 25$

$24 . \quad 15: 45$

$10 . \quad 13: 3.6$

$3 \frac{1}{3} \cdot 16: 18$

345 . $\quad 14: 3$

* $17: 3$

$14 . \quad 15 \div 50$

16. $14: 19$

17. $11: 49$

$\begin{array}{ll}22 & 15: 32 \\ 313 . & 17: 47\end{array}$

5.4

8-14 
ENVIRONMENTAL SUMMARY REPORT

SITE: TAOS STATE OFFICE BUILDING

REPORT PERIOD: $82 / 3$
SITE NUMBER: 186

LATITUDE: 36.50 DEG NORTH
LONGITUDE: 105.50 DEG WEST WIND: NUMBER OF HOURS IN EACH VELOCITY INTERVAL

\begin{tabular}{|c|c|c|c|c|c|c|c|c|c|}
\hline DAY & $\begin{array}{l}0-3 \\
M P H\end{array}$ & $\begin{array}{l}4-7 \\
\text { MPH }\end{array}$ & $\begin{array}{l}8-12 \\
M P H\end{array}$ & $\begin{array}{l}13-18 \\
M P H\end{array}$ & $\begin{array}{l}19-24 \\
M P H\end{array}$ & $\begin{array}{c}25-31 \\
M P H\end{array}$ & $\begin{array}{l}32-38 \\
\mathrm{MPH}\end{array}$ & $\begin{array}{c}A B O V E \\
38\end{array}$ & $\begin{array}{c}\text { MISSING } \\
\text { HOURS }\end{array}$ \\
\hline $\begin{array}{r}1 \\
2 \\
3 \\
4 \\
5 \\
6 \\
7 \\
8 \\
9 \\
10 \\
11 \\
12 \\
13 \\
14 \\
15 \\
16 \\
17 \\
18 \\
19 \\
20 \\
21 \\
22 \\
23 \\
24 \\
25 \\
26 \\
27 \\
28 \\
29 \\
30 \\
31\end{array}$ & $\begin{array}{r}15 \\
13 \\
7 \\
5 \\
13 \\
16 \\
8 \\
13 \\
13 \\
11 \\
15 \\
9 \\
9 \\
10 \\
11 \\
8 \\
12 \\
7 \\
2 \\
11 \\
12 \\
12 \\
11 \\
5 \\
9 \\
16 \\
12 \\
13 \\
5 \\
4 \\
10\end{array}$ & $\begin{array}{l}5 \\
3 \\
3 \\
6 \\
2 \\
1 \\
8 \\
5 \\
5 \\
9 \\
6 \\
6 \\
2 \\
2 \\
0 \\
3 \\
8 \\
2 \\
7 \\
3 \\
5 \\
5 \\
4 \\
5 \\
4 \\
4 \\
6 \\
9 \\
9 \\
9 \\
7 \\
7 \\
3 \\
1 \\
2 \\
5\end{array}$ & 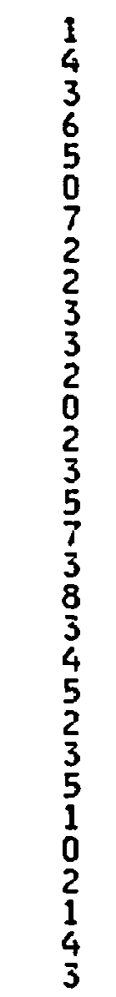 & 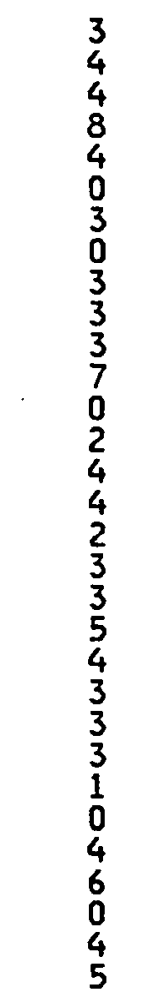 & $\begin{array}{l}0 \\
0 \\
4 \\
3 \\
1 \\
0 \\
1 \\
0 \\
0 \\
1 \\
1 \\
6 \\
0 \\
0 \\
3 \\
0 \\
0 \\
5 \\
5 \\
0 \\
0 \\
0 \\
2 \\
4 \\
0 \\
0 \\
1 \\
0 \\
6 \\
2 \\
0\end{array}$ & $\begin{array}{l}0 \\
0 \\
0 \\
0 \\
0 \\
0 \\
0 \\
0 \\
0 \\
0 \\
0 \\
0 \\
0 \\
0 \\
0 \\
0 \\
0 \\
1 \\
0 \\
0 \\
0 \\
1 \\
2 \\
0 \\
0 \\
0 \\
0 \\
0 \\
0 \\
0 \\
0 \\
0\end{array}$ & $\begin{array}{l}0 \\
0 \\
0 \\
0 \\
0 \\
0 \\
0 \\
0 \\
0\end{array}$ & $\begin{array}{l}0 \\
0 \\
0 \\
0 \\
0 \\
0\end{array}$ & $\begin{array}{r}0 \\
0 \\
0 \\
0 \\
0 \\
0 \\
0 \\
0 \\
0 \\
0 \\
0 \\
0 \\
0 \\
12 \\
2 \\
0 \\
0 \\
0 \\
0 \\
0 \\
0 \\
0 \\
0 \\
0 \\
0 \\
0 \\
0 \\
0 \\
0 \\
0 \\
0 \\
1\end{array}$ \\
\hline AVERAGE & 10.2 & 4.7 & 3.2 & 3.2 & 1.5 & 0.6 & 0.1 & 0.0 & 0.5 \\
\hline TOTAL & 317 & 147 & 99 & 98 & 45 & 19 & 4 & 0 & 15 \\
\hline
\end{tabular}


ENVIRONMENTAL SUMMARY REPORT

SITE: TELLURIDE SCHOOL

REPORT PERIOD: $82 / 3$
SITE NUMBER: 238 LATITUDE: 37.95 DEG NORTH
LONGITUDE: 107.90 DEG WEST

\section{PYRANOMETER TILT: 45 DEG ALTITUDE: 8750 FEET} INSOLATION

DAY TOTAL TOTAL DIFF
INSOLATION INSOLATION IN
BTU/FT2

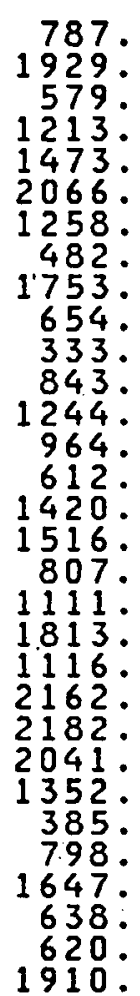

1216.

MEAN

STANDARD

DEVIATION

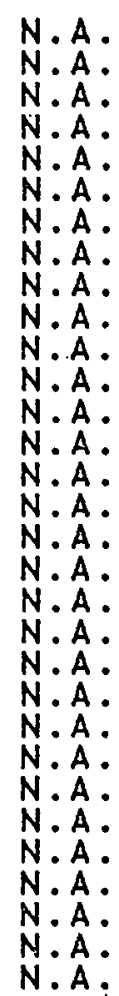

N.A.

N.A. MAXIMUM TOTAL EXTRATERRESTRIAL
INSOLATION
VALUE INSOLATION
BTU/HR/FT2 HR:MN SOLATION BTU/FT2
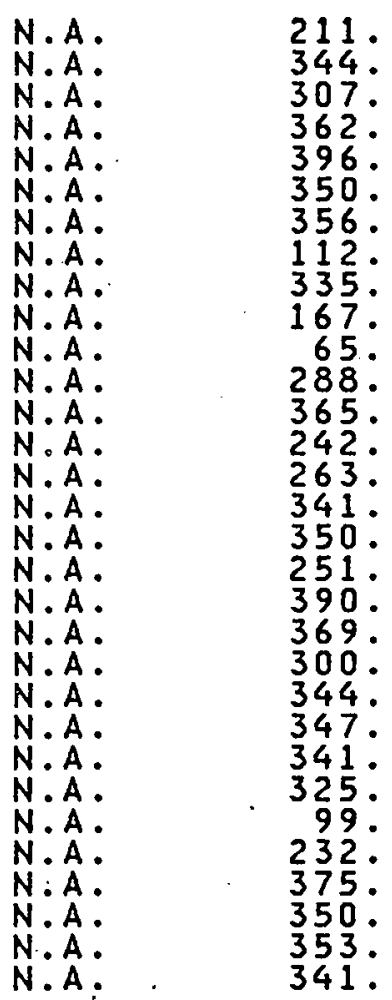

$11: 24$

$14: 5$

$13: 3$

$13: 21$

$13: 58$

$14: 0$

9:29

$13: 53$

$11: 51$

$13: \frac{1}{38}$

$13: 6$

$13: 29$

$12: 29$

14: 0

$13: 5$

$13: 44$

$13: 30$

$14: 57$

$13: 33$

$14: 21$

$14: 31$

$14: 25$

$1 \frac{1}{14}: 5$

$13: 58$

$14: 6$

$15: 48$

$14: 6$

N. A .

N.A.
TTL INS

PERCENT

2868 . 27.

$2879^{\circ} \quad 670^{\circ}$

2896.42 .

2903.051.

$299^{\circ}-43^{\circ}$

2926.116.

$2935.0 \quad 60$.

2950 .

2955.229.

2964.042.

$2970 . \quad 32$

$29780^{\circ} \quad 21$.

2988 . 51.

2996.127.

$3008^{\circ} \quad 60$

$3012 . \quad 37$.

$3016^{\circ}$

3022 .

3028

3032 .

3041 .

3044

3050 .

3050.

3055 .

29.76 .

$\because \therefore 30:$ DER 


\section{ENVIRONMENTAL SUMMARY REPORT}

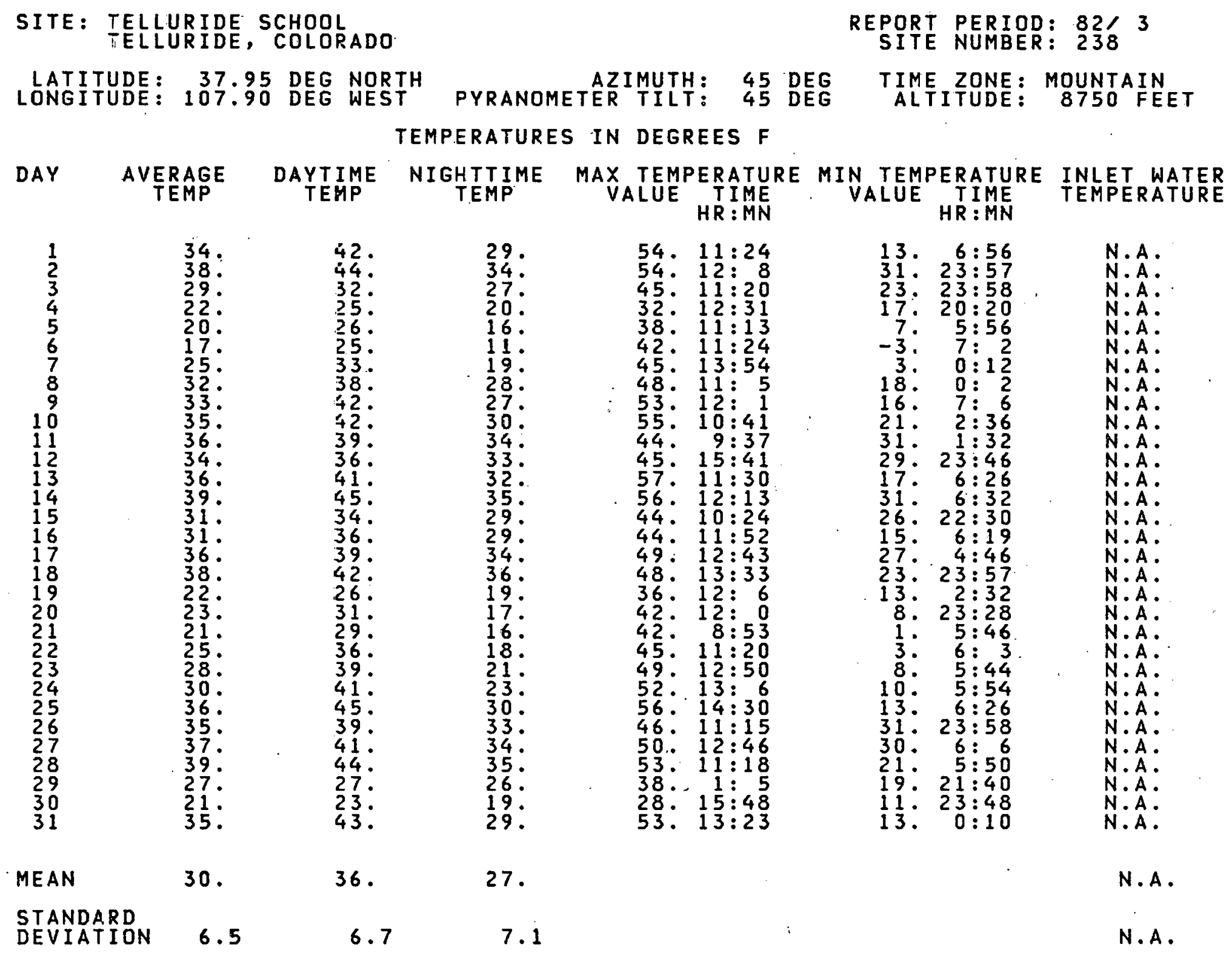


ZONE 9

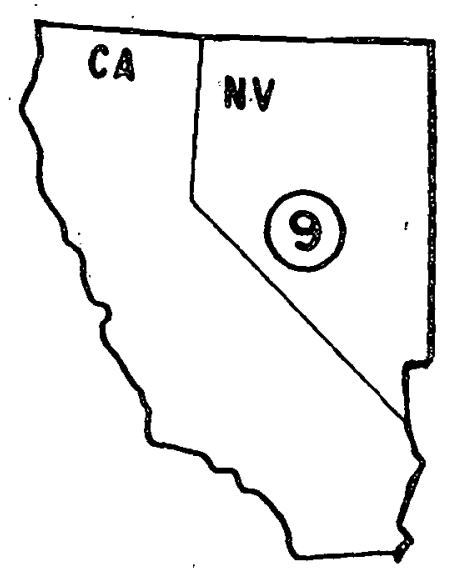




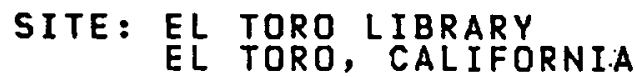

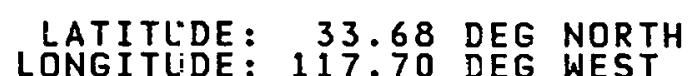
INSOLATION
PYRANOMETER TILT: -30 DEG

REPORT PERIOD: $82 / 3$
SITE NUMBER: 173

TIME ZONE: PACIFIC ALTITUDE: 135 FEET TOTAL
TOTAL DIFF TOTAL DIR
INSOLATION INSOLATION INSOLATION
BTU/FT2

DAY

MAXIMUM TOTAL INSOLATION VALUE TIME BTU $/ H R / F T 2$ HR:MN

EXTRATERRESTRIAL
INSOLATION BTUAFIZ

TTL INS
$\overline{E X T}$ INS
PERCENT

1
2
3
4
5
6
7
8
9
10
11
12
13
14
15
16
17
18
19
20
21
22
23
24
25
26
27
28
29
30
31

326.

1079.

1832.

1828.

1987.

$1600^{\circ}$

1321.

15.

1222.

$1590^{\circ}$.

664 .

522 .

663 .

$327^{\circ}$

1595 .

1848 .

2041.

2016.

3078 .

1900 .

456.

1339 .

$1390^{\circ}$

1319 .

1855.

N.A.

N.A.

N.A.

N.A.

N.A.

N.A.

N.A.

N.A.

N.A.

N.A.

N.A.

N.A.

N.A.

N.A.

N.A.

N.A.

N.A.

N.A.

N.A.

N.A.

N.A.

N.A.

N.A.

N.A.

N.A.

N.A.

N.A.

N.A.

N.A.

N.A

N.A

N.A

N.A.

N.A.

N.A.

N.A.

N.A.

N.A

N.A.

N.A.

N.A.

N.A

N.A

N.A.

N.A.

N.A.

N.A.

N.A

N.A.

N.A

N.A.

N.A.

$115 . \quad 10: 55$

$3190^{\circ} \quad 13: 25$

280 . $\quad 12: 44$

$2690^{\circ} \quad 11: 55$

255. $13: 0$

262. $11: 10$

266. 12:21

143. $11: 20$

$337^{\circ} \quad 11: 5$

183.9952

$165.13: 5$

$190^{\circ} \quad 9: 17$

90. $\quad 12: 36$

359. $\quad 10: 17$

$3450^{\circ} \quad 12: 24$

298. $\quad 12 \vdots 3$

$291 . \quad 12: 28$

298. $11: 36$

$301 . \quad 12: 36$

126. $10: 12$

$2260^{\circ} \quad 14: 42$

276. $14: 27$

$3620^{\circ} \quad 13: 3$

$348.14: 18$

$348 . \quad 11: 44$

N.A.

N.A.

1393.

N.A.

N.A .
2904 . 11.

$\begin{array}{ll}2912 . & 37 . \\ 2928 . & 63 .\end{array}$

2928.

2955 .

2963.

2978 .

2986.

3001.

3013.

3035 :

$3049^{\circ}$

3057 .

3071 .

3082.

3102 .

3116.

3116.

3139.

3145 .

3158.

$3176^{\circ}$

$3192^{\circ}$

3198.

3209 .

3215.

3226.

62.

50.

67.

54.

53.

41.

12 .

22.

$176^{\circ}$

22.

i1.

51 .

59.

65.

63.

$97^{\circ}$

60 .

14 .

42 .

46 .

$4 \frac{1}{8}$.

58 .

3077.

102. 
ENVIRONMENTAL SUMMARY REPORT

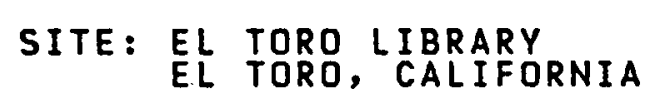

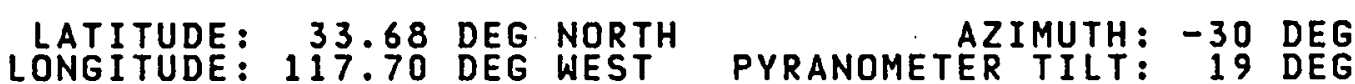

TEMPERATURES IN DEGREES F
REPORT PERIOD: $82 / 3$
SITE. NUMBER: 173

TIME ZONE : PACIFIC

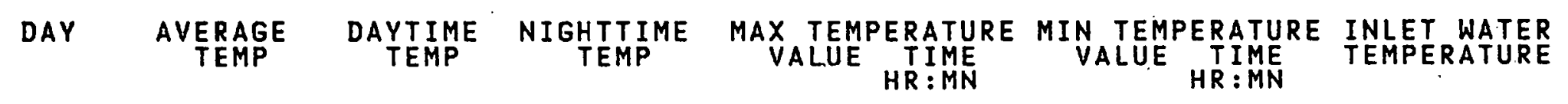

1
2
3
4
5
6
7
8
9
10
11
12
13
14
15
16
17
18
19
20
21
22
23
24
25
26
27
28
29
30
31

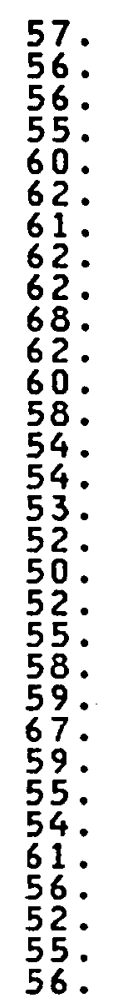

59.

$590^{\circ}$
65.

$\begin{array}{ll}55: & 65: 11: 6 \\ 53: & 67: 14: 45 \\ 50: & 69: 12: 23\end{array}$

$69: 12: 23$

$73.13: 11$

$78 \cdot 12: 59$

$81 \cdot 11: 18$

79 . $10: 11$

80. $11: 22$

86. $13: 38$

$74: 13: 13$

$74: 11: 6$

$65 \cdot 9: 52$

$59 \cdot 8: 33$

$64 \cdot 10: 50$

$56 \cdot 13: 14$

$62: 14: 44$

66 . 14: 6

$74 \cdot 11: 52$

$78 \cdot 11: 56$

$81 \cdot 12: 12$

$78: 13: 50$

$67.10: 12$

62. $14: 42$

74. $14: 16$

$69 \cdot 13: 19$

$65 \cdot 11: 38$

$70 \cdot 11: 28$

53. $23: 38$

$50 \div 4: 20$

45. 6:10

42. $4: 18$

44. $3: 24$

46. $5: 47$

$44 \cdot \quad 4: 6$

$46.23: 58$

53. $2: 34$

$56: 22: 27$

54. $4: 57$

51. $4: 57$

$51 \cdot 16: 54$

$49: 23: 49$

49. $1: 36$

$46.23: 59$

$42.6: 12$

39. $5: 34$

39. $5: 18$

$40.5: 34$

$41 . \quad 5: 21$

43. $5: 7$

$44 . \quad 3: 46$

47. $5: 13$

48. $2: 39$

$47: 23: 41$

$45: 23: 54$

$45: 23: 33$
$40: \quad 5: 33$

N.A.

N.A.

N.A.

N.A.

N.A.

N.A.

NA.

N.A.

N.A.

N.A.

N.A.

N.A.

N.A.

$N \cdot A$.

N.A.

N.A.

N.A.

N.A.

N.A.

N.A.

N.A.

N.A.

N.A.

49.

48 .

N.A.

MEAN

57.

64.

52.

$N \cdot A$.

STANDARD

4.3

6.8

3.6

N.A. 
ENVIRONMENTAL SUMMARY REPORT

SITE: A QUINTA MOTOR INN

REPORT PERIOD: $82 / 3$
SITE NUMBER: 245

LATITUDE: 36.15 DEG NORTH
LONGITUDE: 115.10 DEG WEST PYRANOMETER TILT: 46 DEG INSOLATION

DAY

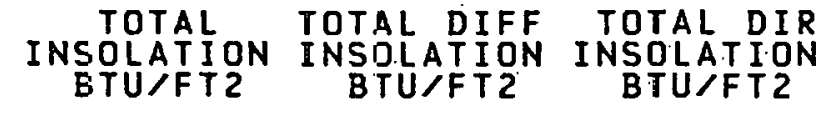

MAXIMUM TOTAL EXTRATERRESTRIAL INSOLATION VALUE TIME
BTU/HR/FT2 HR:MN INSOLATION
BTU/FT2

\section{TTL INS \\ EXT INS} (1)

$N \cdot A$.

N.A.

157. $11: 24$

3349.

1179.

N.A.

2153.

2261 .

2260 .

1314.

1409 .

1772 .

663 .

2103.

2328 .

2345 .

2345 .

$1795^{\circ}$

1278 .

1793.

2134.

$2361^{\circ}$

$2361^{\circ}$

105.

1311.

1592.

2321 .

2052 .

2420.

N.A.

N.A.

N.A.

N.A.

N.A.

N.A.

N.A.

N.A.

N.A.

N.A.

N.A.

N.A.

N.A.

N.A.

N.A.

N.A.

N.A.

N.A.

N.A.

N.A.

N:A.

N.A.

N.A.

N.A.

N.A.

N.A.

N.A.

N.A.

$N \cdot A$

N.A.

N.A.

N.A.

$N \cdot A$

N.A.

N.A.

N.A

N.A.

N.A.

N.A.

N.A

N.A

N.A.

N.A.

N.A.

N.A.

N.A.

N.A.

N.A.

N.A.

$376.12: 45$

$321^{\circ} \quad 12: 50$

$334^{\circ} \quad 13: 18$

334 . $12: 36$

$279^{\circ} \quad 11: 7$

$2650^{\circ} \quad 12: 27$

251. $\quad 11: 49$

153. $15: 32$

$331 . \quad 12: 59$

355. $12: 43$

369 ० $11: 31$

$3410^{\circ} \quad 12: 55$

$360^{\circ} \quad 12: 23$

$359.12: 36$

373. $14: 23$

355: $11: 27$

$338: \quad 12: 56$

334 . $\quad 12: 32$

$331 . \quad 12: 29$

$15: 10$

289. $12: 32$

$366.11: 49$

331 . $12: 42$

359. $\quad 12: 12$

355 . $13: 27$

$348 . \quad 12: 17$

3351 .

3351 .

3352 .

3352 .

3352 .

3352 .

3346 .

3345 .

3343 .

3341 .

3335 .

3332.

3326.

3323 .

3312 .

3307.

3302

3293.

3277 .

3268 .

3261 .

3251 .

3241 .

3231 .

N.A.

N.A.

3314.

STANDARD

DEVIATION 604.

N.A.

N.A.

41. 
ENVIRONMENTAL SUMMARY REPORT

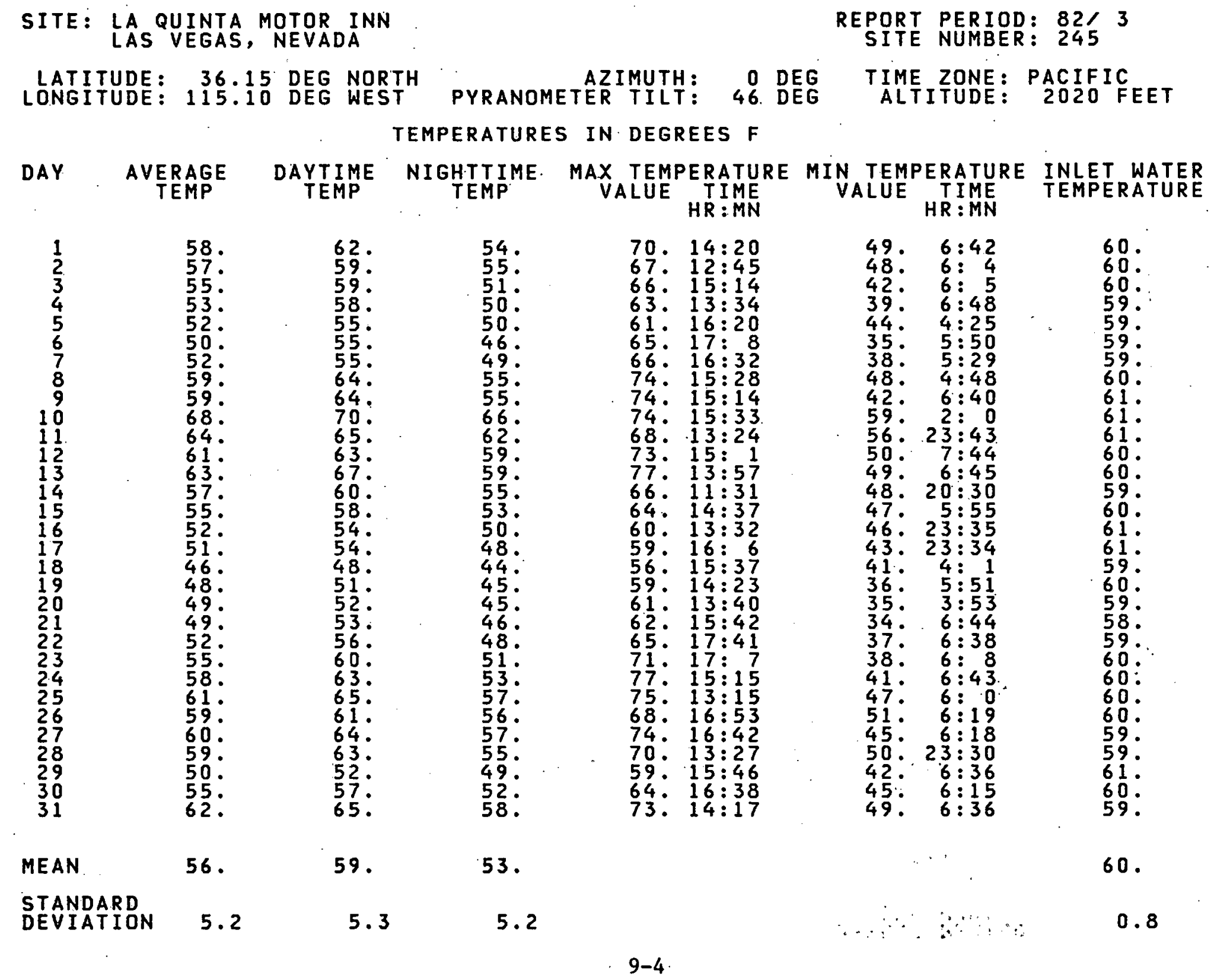


SITE: DAKMEAD

LONTITJDE: 37.20 DEG NORTH PYRANOMETERIMUTH: 40 DEE LONGITIJDE: 121.55 DEG WEST PYRANOMETER TILT: 45 DEG INSOLATION
REPORT PERIOD: $82 / 3$
SITE NUMBER: 197

TIME ZONE: PACIFIC

\begin{tabular}{|c|c|c|c|c|c|c|}
\hline DAY & $\begin{array}{l}\text { TOTAL } \\
\text { INSOLATION } \\
\text { ETU/FT2 }\end{array}$ & $\begin{array}{l}\text { TOTAL DIFF } \\
\text { INSDLATION } \\
\text { BTU/FT2 }\end{array}$ & $\begin{array}{l}\text { TOTAL DIR } \\
\text { INSOLATION } \\
\text { BTU } / F \text { T }\end{array}$ & $\begin{array}{c}\text { MAXIMUM TOTAL } \\
\text { INSOLATION } \\
\text { VALUE TIME } \\
\text { BTU/HR/FT2 HR:MN }\end{array}$ & $\begin{array}{c}\text { EXTRATERRESTRIAL } \\
\text { INSOLATION } \\
\text { BTU/FT2 }\end{array}$ & \begin{tabular}{l} 
TTL INS \\
\hdashline-1 \\
EXT INS \\
PERCENT
\end{tabular} \\
\hline
\end{tabular}

N.A.

305 .

1220

1951.

1981

1897 .

308

2094

579.
759.

759.

1979

948 .

785 .

1297

1108

681.

651
1242

1242 .

2161

2171 .

2085 .

$1794^{\circ}$.

1970 .

353 .

901.

1908 .

664.

N.A.

N.A.

N.A.

N.A.

N.A.

N.A.

N.A.

N.A.

N.A.

N.A.

N.A.

N.A.

N.A.

N.A.

N.A.

N.A.

N.A.

$N \cdot A \cdot$

N.A.

N.A.

N.A.

N.A.

N.A.

N.A.

N.A.

N.A.

204. $13: 6$

3345 .

$333 . \quad 12: 11$

$12: \frac{1}{2} 6$

$327^{\circ}$

$12: 49$

$12: 54$

336.

$11: 36$

107 .

$11: 40$

$12: 40$

197 .

$11: 11$

314.

$12: 16$

272 .

$1 \frac{1}{1}: 23$

310.

$12: 20$

$11: 16$

339.

$11: 44$

$11: 14$

$11: 44$

372 .

$12: 14$

317 .

$1 \frac{1}{2}: 42$

$12: 3$

317 .

$12: 5$

314.

$12: 39$

310 .

$11: 58$

$13: 12$

272 .

304.

$9: 28$

$12: 19$

$207.19: 15$

N.A.

N.A.

$10: 25$

MEAN

1261.

N.A.

N.A.

N.A.

N.A.

9.

3345 .

3348 .

3352 .

3348 .

3349.

3351 .

3351 .

$3346^{\circ}$

$3345^{\circ}$

3344 .

3343 .

$3335^{\circ}$.

3333 .

3327 .

3321 .

3313 .

3305 .

3292 .

3284 .

3278 。

$3252^{\circ}$

36.
58.

STANDAFD

DEVIATION 689.

3326 .

30. 


\section{ENVIRONMENTAL SUMMARY REPORT}

SITE: OAKMEAD

REPORT PERIOD: $82 / 3$
SITE NUMBER: 197

LATITUDE: 37.20 DEG NORTH
LONGITUDE: 121.55 DEG WEST PYRANOMETER TILT: 45 DEG TIME ZONE: PACIFIC

TEMPERATURES IN DEGREES F

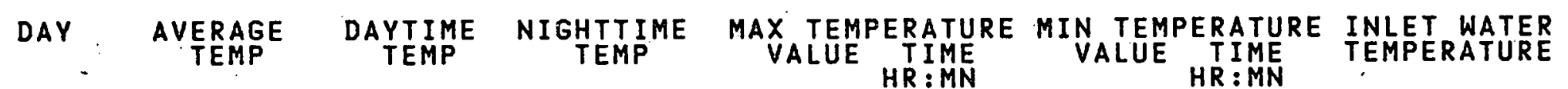

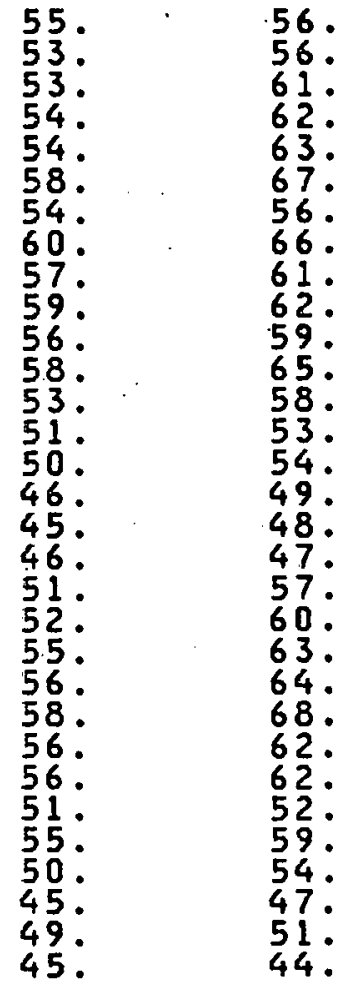

54. $60.10: 10$

$67: 15: 55$

$70 \cdot 115: 10$

$72 \cdot 15: 1 \frac{1}{44}$

$84 \cdot 14: 14$

65. $11: 36$

72. $13: 37$

$67 \cdot 14: 23$

79. $16: 30$

$70 \cdot 16: 20$

71: $12: 22$

65. $12: 30$

60. $13: 34$

57. $13: 57$

$60 \cdot 11: 25$

$68: 12: 20$

$69: 15: 26$

$78: 14: 43$

$74 \cdot 11: 54$

$81 \cdot 13: 27$

$73 \cdot 11: 45$

66 : 11:52

$66.13: 52$

62.115:30

$56 \cdot 12: 41$

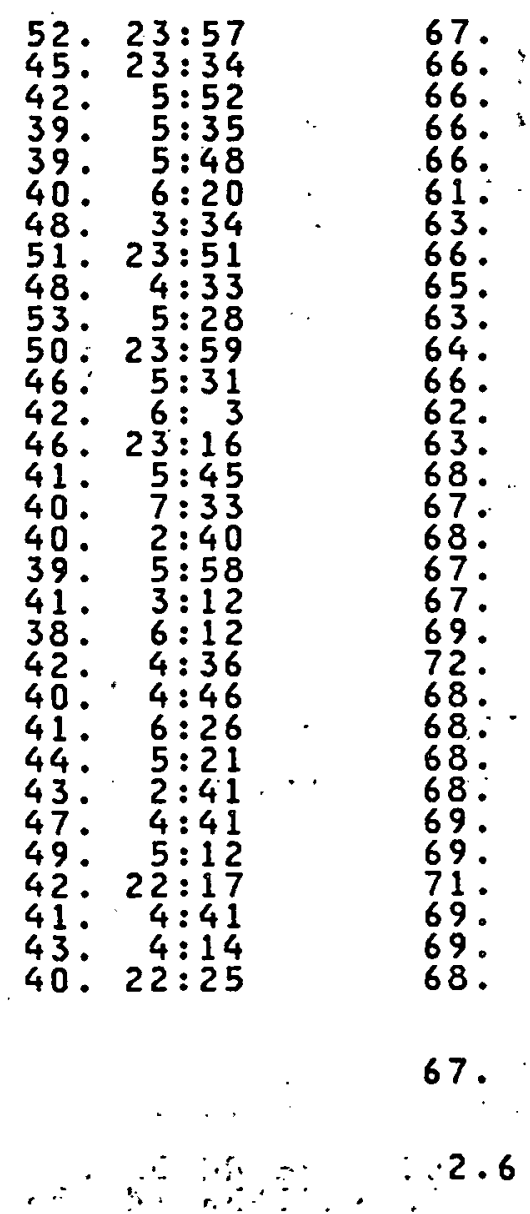

MEĀN

53.

.58.

48.

67.

STANDARD

4.4

.6 .3

3.7 
SITE: OAKMEAD

REPORT PERIOD: $82 / 3$

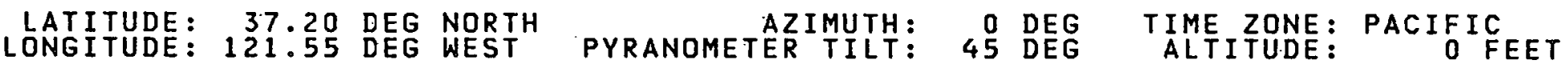

WIND: SPEED IN MPH-DIRECTION IN DEGREES

\begin{tabular}{|c|c|c|c|c|c|c|c|}
\hline DAY & $\begin{array}{l}\text { AVG WIND } \\
\text { DIRECTIDN }\end{array}$ & $\begin{array}{c}\text { AVG WIND } \\
\text { SPEED }\end{array}$ & $\begin{array}{l}\text { DAYTIME } \\
\text { DIRECTION }\end{array}$ & $\begin{array}{l}\text { DAYTIME } \\
\text { SPEED }\end{array}$ & \multicolumn{3}{|c|}{ SPEED MAXIMUM WIND } \\
\hline $\begin{array}{l}1 \\
2 \\
3 \\
4 \\
5 \\
6 \\
7 \\
8 \\
9 \\
10 \\
11 \\
12 \\
13 \\
14 \\
15 \\
16 \\
17 \\
18 \\
19 \\
20 \\
21 \\
22 \\
23 \\
24 \\
25 \\
26 \\
27 \\
28 \\
29 \\
30 \\
31\end{array}$ & $\begin{array}{r}161 \\
223 \\
* \\
* \\
* \\
* \\
* \\
154 \\
187 \\
* \\
163 . \\
* \\
* \\
* \\
29 \\
290 \\
261 . \\
151 . \\
174 \\
258 . \\
* \\
* \\
* \\
* \\
* \\
* \\
* \\
* \\
* \\
* \\
* \\
220 \\
185 \\
157 . \\
146 .\end{array}$ & $\begin{array}{l}6: \\
3: \\
1: \\
1: \\
1: \\
\frac{1}{2}: \\
3: \\
2: \\
3: \\
2: \\
1: \\
\frac{1}{3}: \\
3: \\
6: \\
3: \\
2: \\
1: \\
1: \\
1: \\
1: \\
\frac{1}{2}: \\
2: \\
1: \\
2: \\
3: \\
6: \\
6: \\
5:\end{array}$ & $\begin{array}{r}159 . \\
244 \\
* \\
* \\
* \\
* \\
* \\
160 \\
194 . \\
* \\
175 \\
* \\
* \\
* \\
* \\
300 \\
285 \\
155 \\
222 . \\
270 \\
* \\
* \\
* \\
* \\
* \\
* \\
* \\
* \\
* \\
* \\
* \\
259 \\
182 \\
154 \\
160 .\end{array}$ & 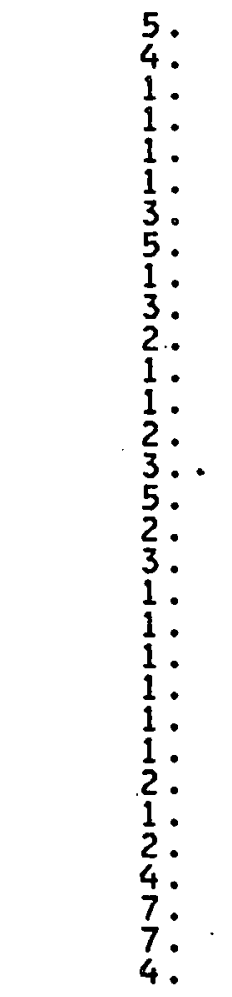 & 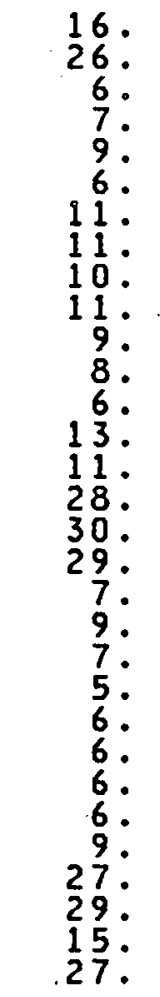 & $\begin{array}{r}163 \\
134 \\
* \\
* \\
* \\
* \\
* \\
138 \\
207 . \\
210^{*} \\
* \\
* \\
* \\
257^{*} \\
291 . \\
207 \\
184 . \\
16 . \\
* \\
* \\
* \\
* \\
* \\
* \\
* \\
* \\
* \\
* \\
* \\
166 . \\
150 . \\
189 . \\
161 .\end{array}$ & 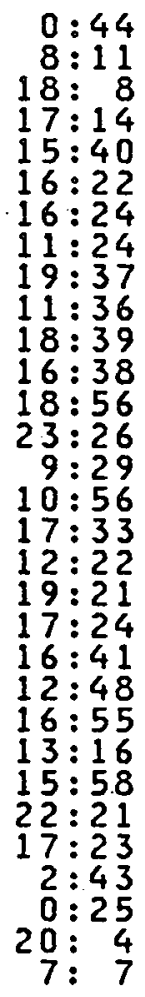 \\
\hline MEAN & 181. & 2. & 201. & 3. & $\therefore$ & & \\
\hline $\begin{array}{l}T A N D \\
\text { EVI }\end{array}$ & $49 . .4$ & 1.7 & 53.9 & 1.8 & & & \\
\hline
\end{tabular}


ENVIRONMENTAL SUMMARY REPORT

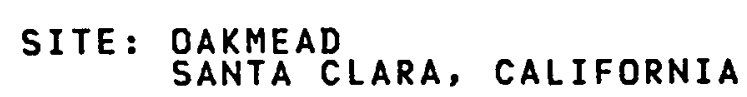

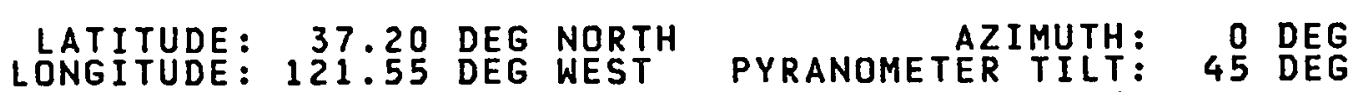

REPORT PERIOD: $82 / 3$ SITE NUMBER: 197

WIND: NUMBER OF HOURS IN EACH VELOCITY INTERVAL

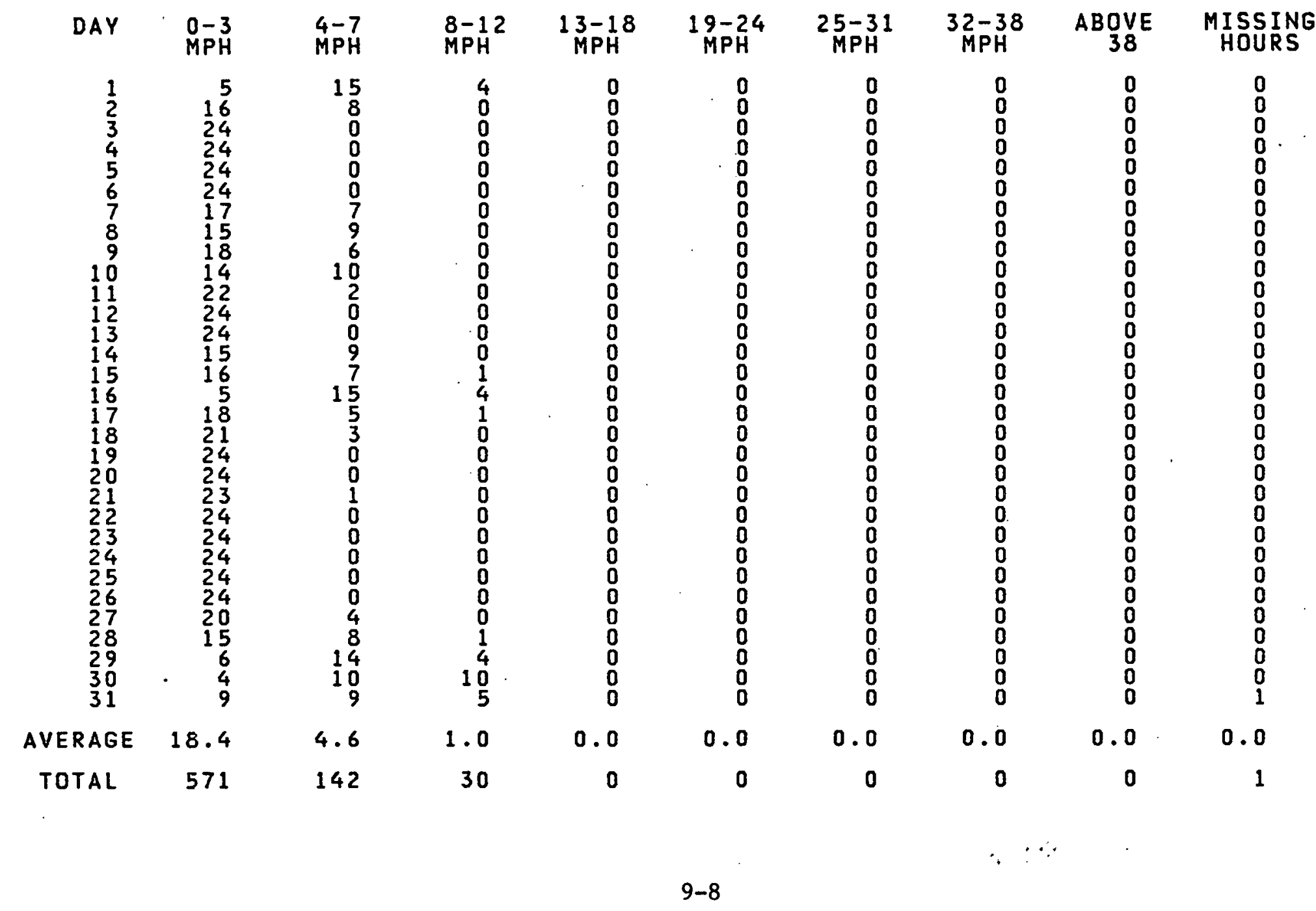


ENVIRONMENTAL SUMMARY REPORT

SITE: SAN ANSELMO SCHOOL

REPORT PERIOD: $82 / 3$
SITE NUMBER: 145

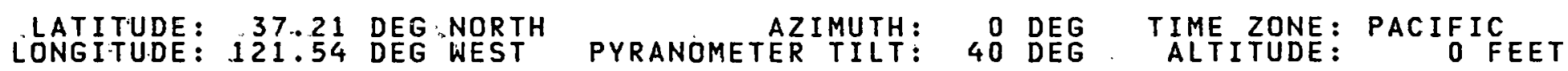
INSOLATION

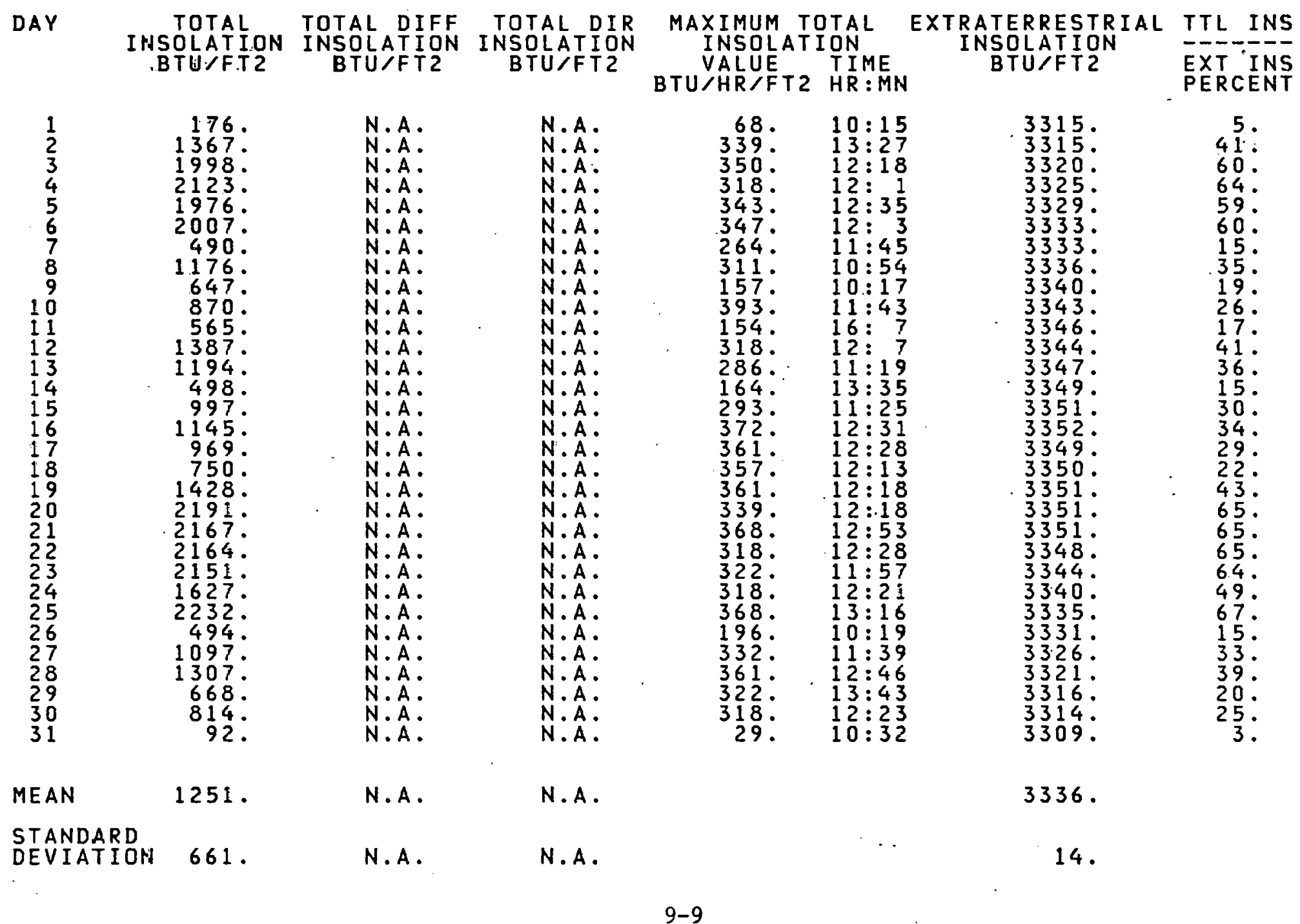


ENVIRONMENTAL SUMMARY REPORT

SITE: SAN ANSELMO SCHOOL

REPORT PERIOD: $82 / 3$
SITE NUMBER: 145

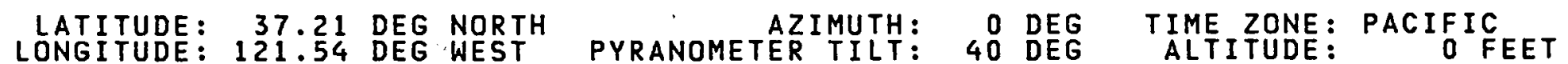

TEMPERATURES IN DEGREES F

\begin{tabular}{|c|c|c|c|c|c|c|}
\hline DAY & $\begin{array}{c}\text { AVERAGE. } \\
\text { TEMP }\end{array}$ & $\begin{array}{c}\text { DAYTIME } \\
\text { TEMP }\end{array}$ & $\underset{\text { TEMP }}{\text { NIGT IME }}$ & $\begin{array}{c}\text { MAX TEMPERATURE } \\
\text { VALUE TIME } \\
\text { HR:MN }\end{array}$ & $\begin{array}{c}\text { MIN TEMPERATURE } \\
\text { VALUE TIME } \\
\text { HR:MN }\end{array}$ & $\begin{array}{l}\text { INLET WATER } \\
\text { TEMPERATURE }\end{array}$ \\
\hline
\end{tabular}

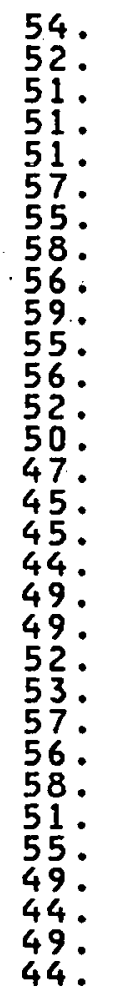

$5 \dot{5}$

52.

59: $10: 31$

64:-14:10

$64 \cdot 11: 51$

70:15:26

78. $15: 31$

$68 \cdot 11: 50$

$74: 1 \frac{1}{70}: 2 \frac{2}{3}$

73: $11: 38$

64. $17: 11$

$6 \frac{71}{67}: 12: 30$

$67 \cdot 12: 39$

$55: 14: 23$

$57: 14: 33$

57. 12:39

53. $17: 12$

64. $12: 45$

$66 \cdot 14: 47$

72. $12: 53$

$78 \cdot 14: 4 \frac{2}{3}$

$78 \cdot 14: 43$

$73 \cdot 12: 31$

74. $12: 42$

69: $11: 44$

$60: 12: 46$

$56: 14: 49$

$63: 12: 23$

MEAN

52.

57.

47.

49. $22: 36$

$22: 58$

$39.6: 10$

$37: \quad 5: 28$

$41^{\circ} \cdot \cdots, 1: 28$

48. $2: 0$

$51 \cdot 21: 34$

45: $6: 7$

49:23: 3

$46: 23: 46$

40 . $6: 26$

$45 \cdot 23: 59$

$36.6: 21$

$39.6: 44$

$38 . \quad 3: 53$

$39: 23: 57$

$35: 4: 34$

39 . $1: 54$

$37: \quad 6: 25$

38.60

$47.2: 37$

$40 . \quad 5: 55$

$47 \cdot \quad 2: 38$

$40.21: 29$

39 : $6: 11$

$41.6: \frac{1}{3}$

39: $23: 47$

N.A.

N.A.

N.A.

N.A.

N.A.

N.A.

N.A.

N.A.

N.A

N.A

N.A.

N.A.

N.A.

N.A.

N.A.

N.A.

N.A.

N.A.

$N$. A.

N.A.

N.A.

N.A.

N.A.

N.A.

N.A.

N.A.

N.A

N.A.

$N: A$

N.A.

45.

51. 7:52

STANDARD DEVIATION

4.6

6.1

4.0

N.A.

N. A . 
ENVIRONMENTAL SUMMARY REPORT

SITE: SAN ANSELMO SCHOOL

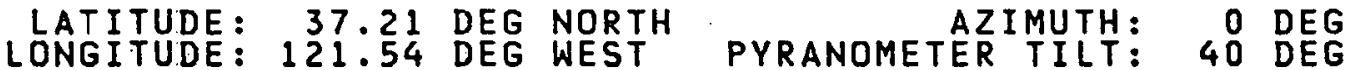

RELATIVE HUMIDITY IN PER CENT

DAY
D
1
2
2
3
4
5
6
7
8
9
10
11
12
13
14
15
16
17
18
19
20
21
22
23
24
25
26
27
28
29
30
31
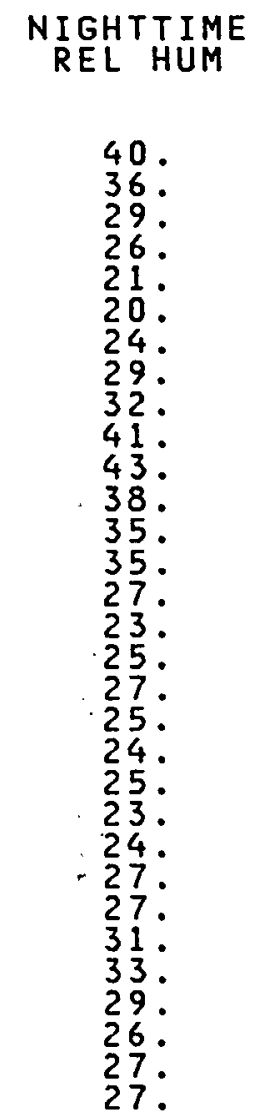

29.

\begin{tabular}{|c|c|c|}
\hline $\begin{array}{l}1 \\
2 \\
3 \\
4 \\
5 \\
6 \\
7 \\
8 \\
9 \\
0 \\
1 \\
2 \\
3 \\
4 \\
5 \\
5 \\
6 \\
7 \\
8 \\
9 \\
0 \\
1 \\
2 \\
3\end{array}$ & 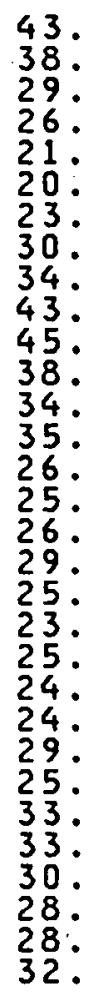 & 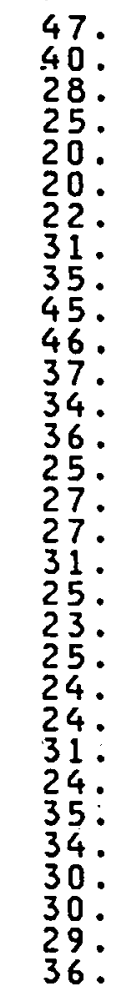 \\
\hline & 30. & $\therefore 30$. \\
\hline
\end{tabular}

$24 . \quad 0: 1$
REPORT PERIOD: $82 / 3$
SITE NUMBER: 145 TIME ZONE: PACIFIC

MAXIMUM HUMIDITY MINIMUM HUMIDITY
VALUE
HRIME

49. $\quad 13: 16$

34. $\quad 9: 59$

29. $\quad 9: 16$

$19: 58$

$21: 32$

$11: 32$

$11: 32$

$11: 14$

$11: 14$

$0: 1 \frac{12}{3}$

$17: 41$

$0: 4$

$9: 45$

$12: 23$

$12: 35$

$9: 38$

$19: 99$

$23: 11$

9: 0

$13: 17$

$15: 7$

$13: 52$

$9: 13$

$14: 17$

$10: 4$

$8: 51$

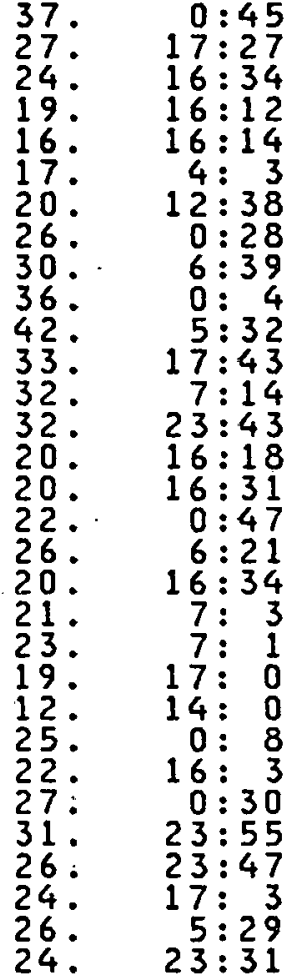


ZONE 10

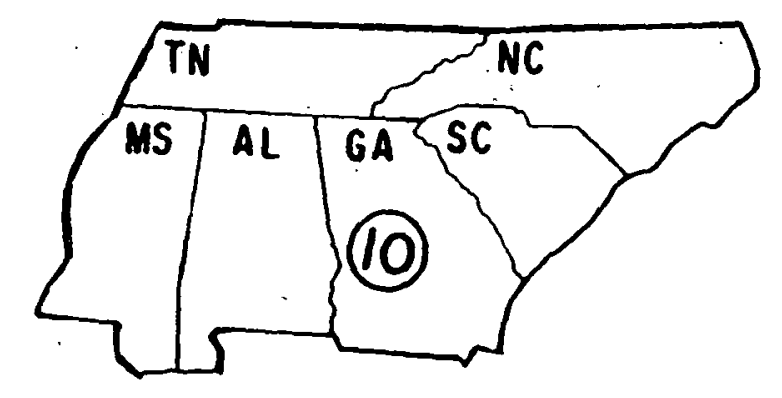


NO VALID DATA

10-1 
ZONE 11

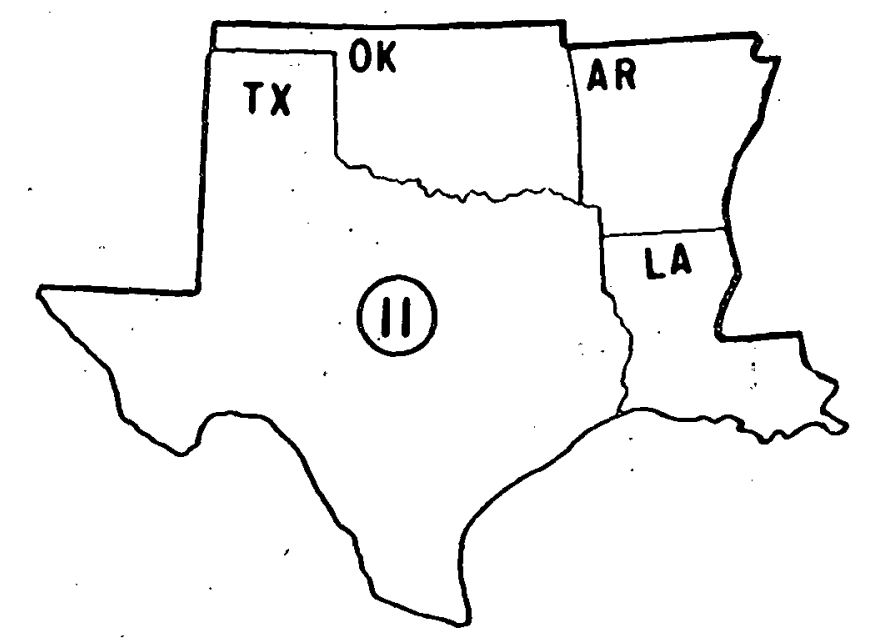


ENVIRONMENTAL SUMMARY REPORT

SITE: CRAFTSMAN ENTERPRISES

LATITUDE: 32.75 DEG NORTH LONGITUDE: 96.70 DEG WEST
PYRANOMETER TILT: $\quad 27$ DEG INSOLATION
REPORT PERIOD: $82 / 3$

TIME ZONE : CENTRAL

ALTITUDE : 551 FEET
DAY TOTAL TOTAL DIFF
INSOLATION INSOLATION
BTU/FT2

2034.

1522 .

1414.

176.

1506 .

2149

2034.

1042 .

1042.

1156.

752 :

1646 .

656 .

1250

13
934

632 .

$151 \frac{1}{15}$

423.

2023.

$880^{\circ}$

816.

1306 .

$88^{\circ}$

1460

1456 .

2037 .

1213.

MEAN

STANDARD

580.
N.A.

N.A.

N.A.

N.A.

N.A.

N.A.

N.A.

N.A.

N.A.

N.A.

N.A.

N.A.

N.A.

N.A.

N.A.

N.A.

N.A.

N.A.

N.A.

N.A.

N.A.

N.A.

N.A.

N.A.

N.A.

N.A.

N.A.

N.A.

N.A.
MAXIMUM TOTAL INSOLATION VALUE TIME

EXTRATERRESTRIAL INSOLATION BTU/FT2

\section{BTU/HR/FT2 HR:MN}

N.A.

N.A.

N.A:

N.A.

N.A:

N.A.

N.A.

N.A.

N.A:

N.A.

N.A.

N.A.

N.A.

N.A.

N.A:

N.A.

N.A.

N.A.

N.A:

N.A.

N.A.

N.A.

N.A:

N.A.

N.A.

$309 . \quad 12: 22$

306

$12: 22$

$313 . \quad 13: 11$

$12: 40$

$11: 23$

$12: \frac{1}{3}$

$12: 20$

$14: 40$

$12: 57$

$11: 37$

$12: 25$

$10: 44$

$12: 21$

$14: 11$

$10: 41$

$12: 34$

$12: 32$

$11: 59$

$12: 47$

$14: 43$

$11: 57$

$14: 21$

$13: 40$

11:51

$13: 43$

13
$11: 52$

$325 . \quad 11: 19$

N.A.

N.A.
$3212 . \quad 63$.

3223 :

3234 .

3245 .

3252 .

3255 .

3265 .

3275 .

3281 .

3293

3298 .

3307 .

3309

3314

3323

3327 .

3335

4337 .

(3).

$3341^{\circ}$.

3340 .

3342 .

3343 .

3344 .

3345 .

3346 .

3299.

42.

$44^{\circ}$.

64.

46.

66.

62.

32

35.

23.

50.

20.

48.

28

19.

13.

61.

26

27.

39 .

44 .

12.

61 .

TTL INS

EXT INS 
ENVIRONMENTAL SUMMARY REPORT

SITE: CRAFTSMAN ENTERPRISES
DALLAS, TEXAS

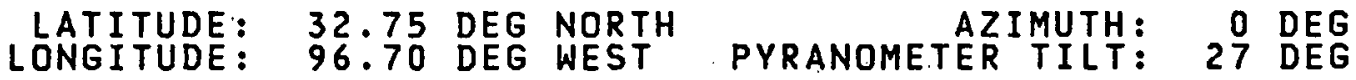

TEMPERATURES IN DEGREES F
REPORT PERIOD: $82 / 3$

SITE NUMBER: 249

TIME ZONE: CENTRAL

ALTITUDE: 551 FEET

\begin{tabular}{|c|c|c|c|c|c|c|}
\hline DA.Y & $\begin{array}{c}\text { AVERAGE } \\
\text { TEMP }\end{array}$ & $\begin{array}{l}\text { DAYTIME } \\
\text { TEMP }\end{array}$ & NIGHTTIME & $\begin{array}{c}\text { MAX TEMPERATURE } \\
\text { VALUE TIME } \\
H R: M N\end{array}$ & $\begin{array}{c}\text { MIN TEMPERATURE } \\
\text { VALUE TIME } \\
\text { HR:MN }\end{array}$ & $\begin{array}{l}\text { INLET WATER } \\
\text { TEMPERATURE }\end{array}$ \\
\hline
\end{tabular}

53
$60:$
$65:$
$48:$
$36:$
$38:$
$45:$
$56:$
$60:$
$66:$
$70:$
$73:$
$70:$
$70:$
$71:$
$73:$
$76:$
$76:$
$76:$
720
$55:$
$55:$
$59:$
$63:$
5.70
$48:$
$41:$
$50:$
$56:$
$71:$
70.

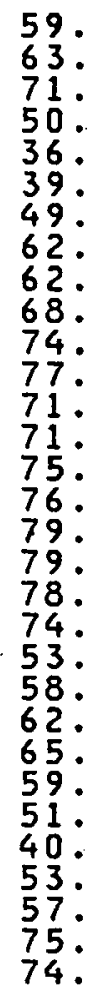

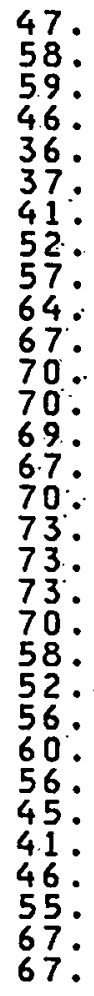

73. $16: 33$

$74 \cdot 1.5: 12$

$86: 14: 58$

$59 \cdot 16: 40$

$47: 16: 54$

$62: 14: 47$

$71 \cdot 16: 41$

$74 \cdot 14: 40$

76. $15: 55$

$81 \cdot 14: 23$

79: $15: 32$

$77 \cdot 15: 55$

$80^{\circ} 12: 55$

$86: 12: 14$

$86 \cdot 14: 14$

$87 \cdot 12: 55$

$89 \cdot 14: 40$

$82 \cdot 13: 56$

66. $0: 9$

$70 \cdot 14 \vdots 329$

$72 \cdot 16: 13$

$72.16: 13$

$63 \cdot 13: 56$

$43 \cdot 15: 14$

62. $13: 44$

63. $22: 48$

86. $14: 36$

MEAN

61.

63.

58.

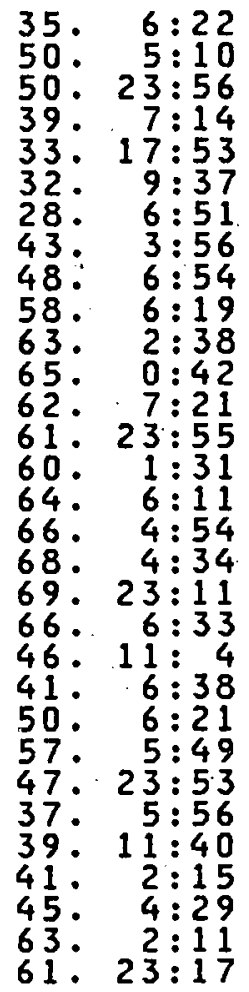

49.

49.

54.

STANDARD

11.8

12.4

11.3

4.9 
ZONE 12

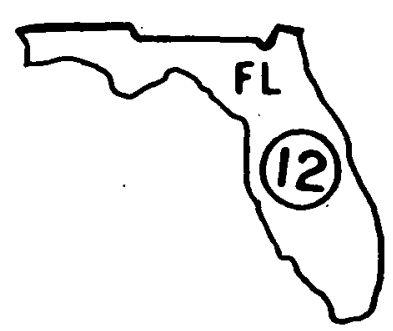


ENVIRONMENTAL SUMMARY REPORT

SITE: FLORIDA SOLAR ENERGY CENTER
CAPE CANAVERAL, FLORIDA

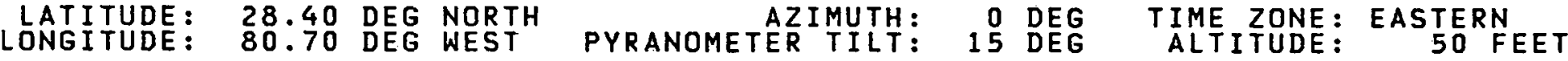 INSOLATION}

REPORT PERIOD: $82 / 3$
SITE NUMBER: 235

\begin{tabular}{|c|c|c|c|c|c|c|}
\hline DAY & $\begin{array}{l}\text { TOTAL } \\
\text { INSOLATION } \\
\text { BTU/FT2 }\end{array}$ & $\begin{array}{l}\text { TOTAL DIFF } \\
\text { INSOLATION } \\
\text { ETU/FT2 }\end{array}$ & $\begin{array}{l}\text { TOTAL'DIR } \\
\text { INSOLATION } \\
\text { BTU/FT2 }\end{array}$ & $\begin{array}{c}\text { MAXIMUM TOTAL } \\
\text { INSOLATION } \\
\text { VALUE } \\
\text { BTU/HIR /FT2 HR:MN }\end{array}$ & $\begin{array}{c}\text { EXTRATERRESTRIAL } \\
\text { INSOLATION } \\
\text { BTU IFT2 }\end{array}$ & $\begin{array}{l}\text { TTL INS } \\
\overline{E X T} \text { INS } \\
\text { PERCENT }\end{array}$ \\
\hline $\begin{array}{l}1 \\
2 \\
3 \\
4 \\
5 \\
6 \\
7 \\
8 \\
9 \\
10 \\
11 \\
12 \\
13 \\
14 \\
15 \\
16 \\
17 \\
18 \\
19 \\
20 \\
21 \\
22 \\
23 \\
24 \\
25 \\
26 \\
27 \\
28 \\
29 \\
30 \\
31\end{array}$ & 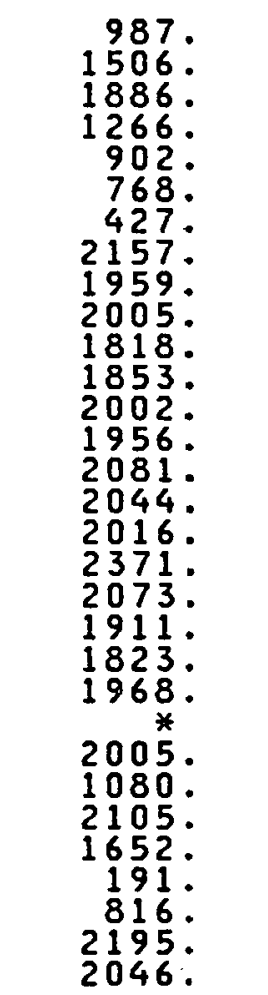 & 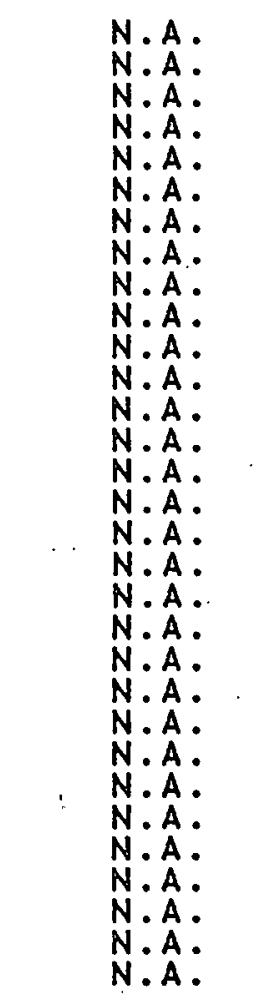 & 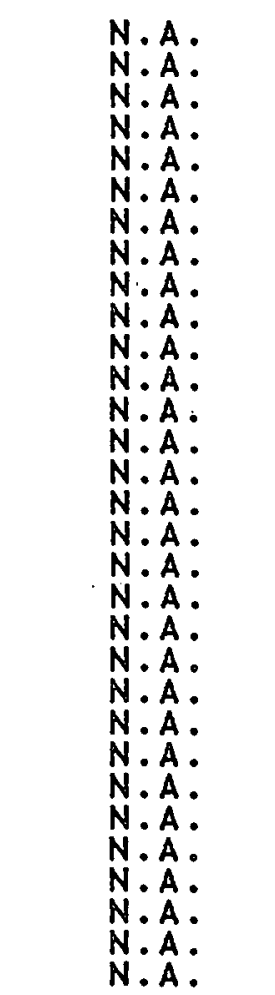 & $\begin{array}{l}12: 49 \\
111: 20 \\
12: 17 \\
13: 46 \\
13: 11 \\
14: 37 \\
10: 31 \\
12: 34 \\
11157 \\
12: 28 \\
11: 42 \\
12: 38 \\
12: 27 \\
13: 12 \\
12: 17 \\
12: 17 \\
12: 18 \\
12: 22 \\
12: 23 \\
12: 29 \\
12: 32 . \\
11: 42 \\
*: * \\
11: 50 \\
14: 54 \\
13: 55 \\
12: 11 \\
9: 32 \\
11: 29 \\
12: 5 \\
111: 18\end{array}$ & 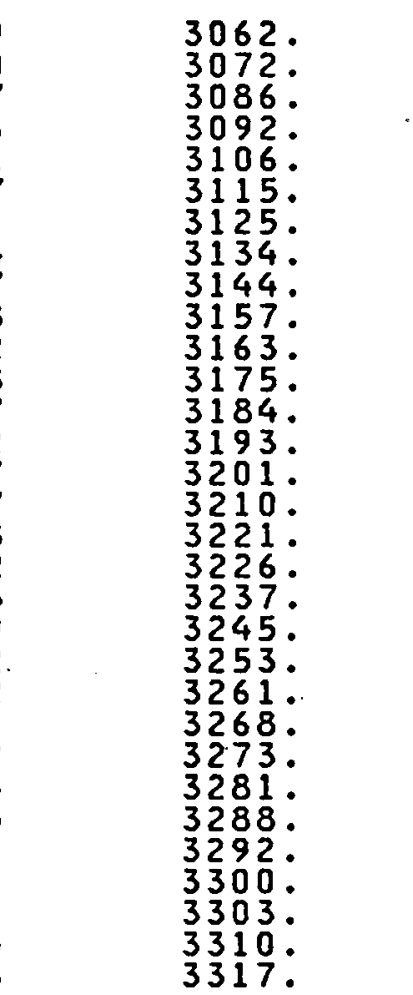 & 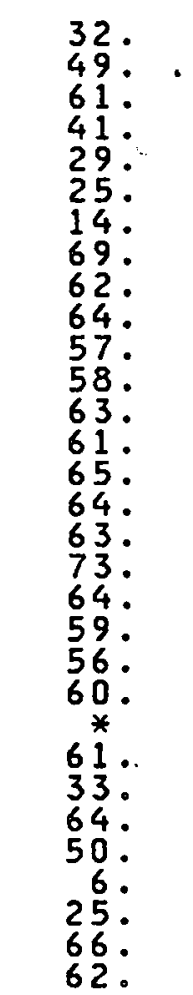 \\
\hline MEAN & 1662. & N.A. & N. A. & & 3203. & \\
\hline
\end{tabular}

STANDARD

DEVIATION 574.

N.A.

N. A .

78 . 
